\title{
Existência e Destruição de Toros Invariante, Para Uma Certa Família de Sistemas Hamiltonianos no R4
}

\author{
Julio Cezar de Oliveira Andrade
}

TESE APRESENTADA

AO,
Instituto de MATEMÁtica E EstatísticA

DA

Universidade DE SÃo PAUlo

PARA

OBTENÇÃ O DO TÍTULO

DE

Doutor EM Cî̂nCIAS

\author{
Programa: Matemática Aplicada \\ Orientador: Prof. Dr. Manuel Valentim de Pera Garcia \\ Coorientador: Prof. Dr. Mário Jorge Dias Carneiro
}

Durante o desenvolvimento deste trabalho o autor recebeu auxílio financeiro do $\mathrm{CNPq}$

São Paulo, 2019 


\section{Existência e Destruição de Toros Invariante, Para Uma Certa Família de Sistemas Hamiltonianos no $\mathbb{R}^{4}$}

Esta versão da tese contém as correções e alterações sugeridas pela Comissão Julgadora durante a defesa da versão original do trabalho, realizada em 07/06/2019. Uma cópia da versão original está disponível no Instituto de Matemática e Estatística da Universidade de São Paulo.

Comissão Julgadora:

- Prof. Dr. Manuel Valentim Garcia (orientador)-IME-USP

- Prof. Dr. Mario Jorge Dias Carneiro - UFMG

- Prof. Dr. Clodoaldo Grotta Ragazzo - IME-USP

- Prof. Dr. Pedro Antonio Santoro Salomão - IME-USP

- Prof. Dr. Ricardo Miranda Martins - UNICAMP 



\section{AGRADECIMENTOS}

A Deus, primeiramente.

À minha família, especialmente aos meus pais, Lovidio e Detinha, ao tio Baial e ao amor da minha vida, a Dani.

Aos meus orientadores Manuel Valentim de Pera Garcia e Mario Jorge Dias Carneiro, por todo apoio, paciência e por acreditarem em mim. Agradeço à Professora Sonia Regina Leite Garcia pelo constante apoio, desde a graduação até hoje. Agradeço ao Professor Jorge Sotomayor por sempre me incentivar e pelas monitorias.

Aos membros da banca, Clodoaldo Ragazzo, Pedro Salomão e Ricardo Miranda, pelas dúvidas respondidas, sugestões e incentivos.

Aos Professores que, mesmo sem me conhecer, respondiam os meus emails ao longo desses anos de estudo, entre eles, Marie-Claude Arnaud, Alfonso Sorrentino, Henrique Bursztyn, Daniel Tausk e Ivan Struchiner.

Ao CNPq, pelo apoio financeiro.

A todos que, direta ou indiretamente, me ajudaram a alcançar este objetivo. 


\section{RESUMO}

ANDRADE, J. C. de O. Existência e Destruição de Toros Invariante, Para Uma Certa Família de Sistemas Hamiltonianos no $\mathbb{R}^{4}$. Tese (Doutorado) - Instituto de Matemática e Estatística, Universidade de São Paulo, São Paulo, 2019.

Estudaremos uma família de sistemas hamiltonianos no $\mathbb{R}^{4}, H_{\epsilon}: \mathbb{R}^{4} \rightarrow \mathbb{R}$, satisfazendo certas condições, dependendo de um parâmetro $\epsilon$. Iremos caracterizar algumas condições sobre níveis de energia desse sistema, que nos permitem concluir existência e destruição de toros invariantes, em tais níveis de energia. Além disso, podemos concluir que o fluxo hamiltoniano, restrito a esses níveis de energia, possui entropia topológica positiva.

Palavras-chave: Sistemas Hamiltonianos, nível de energia, toros invariantes, aplicações twist. 


\begin{abstract}
ANDRADE, J. C. de O. Existence and Destruction of Invariant Torus, For a Certain Family of Hamiltonian Systems in $\mathbb{R}^{4}$. Tese (Doutorado) - Instituto de Matemática e Estatística, Universidade de São Paulo, São Paulo, 2019.

We will study a family of Hamiltonian Systems in $\mathbb{R}^{4}$, satisfyng certain conditions, $H_{\epsilon}: \mathbb{R}^{4} \rightarrow \mathbb{R}$, depending of a parameter $\epsilon$. We will characterize some conditions about the energy levels of this system, which allow us to conclude existence and destruction of invariant torus, at such energy levels. Moreover, we can conclude that the hamiltonian flow, restricted to these energy level, has positive topological entropy.
\end{abstract}

Keywords: Hamiltonian Systems, energy level, invariant torus, twist maps. 


\section{Sumário}

1 Introdução $\quad 3$

1.1 Resultados Principais . . . . . . . . . . . . . . . . . 3

2 Teoria de Sistemas Hamiltonianos. $\quad 9$

2.1 Sistemas Hamiltonianos . . . . . . . . . . . . . . . . . . . . . 9

2.2 Geometria Simplética . . . . . . . . . . . . . . . 16

2.3 Análise de Órbitas Periódicas . . . . . . . . . . . . . 21

2.4 Aplicações Twist Simpléticas . . . . . . . . . . . . . . . . 25

2.5 Teoria de Aubry Mather . . . . . . . . . . . . . . . 31

3 Propriedades Dinâmicas de $X_{H_{\epsilon}} \quad 37$

3.1 Alguns resultados relacionados. . . . . . . . . . . . . . . 37

3.2 Demonstração da Proposição 3.1.1 . . . . . . . . . . . . . . . 41

3.3 Construção da Transformação de Poincaré . . . . . . . . . . . 47

3.4 Algumas Questões . . . . . . . . . . . . . . . 50

4 Mudança Conveniente de Coordenadas. 53

4.1 A Forma Normal de Birkhoff . . . . . . . . . . . . . . . . . 53

4.2 Aplicando o Teorema 4.1.1 às Aplicações de Poincaré . . . . . 56

4.3 Algumas Questões em coordenadas twist. . . . . . . . . . . 57

5 O Primeiro Invariante de Birkhoff $\quad 59$

5.1 Fórmula para o Primeiro Invariante de Birkhoff. . . . . . . . . 59

5.2 A Aplicação $\psi_{0}$ de uma forma conveniente. . . . . . . . . . . . 61

5.3 Análise do Primeiro Invariante de Birkhoff para $\psi_{0} \ldots \ldots$. . . . 65

6 Destruição de Curvas Invariantes. $\quad 69$

6.1 Critério de Melnikov . . . . . . . . . . . . . . . . 69

6.2 Critério de Melnikov Atraves da Função Geradora. . . . . . . . 73 
7 A Função de Melnikov na Seção $\quad 75$

7.1 Algumas considerações iniciais. . . . . . . . . . . . . . . 75

7.2 Análise do termo $\bar{M}_{1} \ldots \ldots \ldots$. . . . . . . . . . 77

7.3 Análise do termo $\bar{N}_{1} \ldots \ldots \ldots$. . . . . . . . . 82

7.4 Algumas observações sobre a função $\bar{L}_{1} \ldots$. . . . . . . . . . . 83

7.5 Análise da Função $A_{1}$. . . . . . . . . . . . . . . . . . . . . 85

7.6 As Derivadas da Função $A_{1}$. . . . . . . . . . . . . . . 90

8 Destruição de Curvas Invariantes. $\quad 93$

8.1 A condição de Melnikov a partir das soluções explícitas de $X_{H_{1}} .93$

8.2 Análise do termo relacionado a $\mu_{1} \ldots \ldots$. . . . . . . . . . 98

8.3 As singularidades da função $\mu_{1}$. . . . . . . . . . . . . . . . . 102

8.4 A Contribuição de $\mu_{1}$ ao Somatório. . . . . . . . . . . . . . . . 110

8.5 Conclusão a respeito da destruição de curvas invariantes . . . 116

8.6 Algumas Observações Finais . . . . . . . . . . . . . . . . . . . 118

A As derivadas do campo $f_{1}$. 121

A.1 Reescrevendo as Transformações de Poincaré . . . . . . . . . . 121

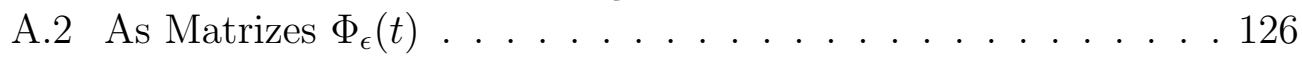

A.3 Voltando a derivada de $\bar{G}_{1} \ldots \ldots \ldots$. . . . . . . . . . . 131

A.4 Análise dos Coeficientes da Equação A.10. . . . . . . . . . . . 133

A.5 Cálculo das derivadas de $A_{1} \ldots \ldots$. . . . . . . . . . . 139

B Análise da subvariedade $M_{2} \quad 143$ 


\section{Capítulo 1}

\section{Introdução}

Neste capítulo, introduzimos a família de sistemas hamiltonianos que estudaremos neste texto e apresentamos os enunciados dos resultados principais.

\subsection{Resultados Principais}

Consideremos a seguinte família de Hamiltonianas, $H_{\epsilon}: \mathbb{R}^{4} \rightarrow \mathbb{R}$, dependendo de um parâmetro $\epsilon$, dada por:

$$
H_{\epsilon}\left(x_{1}, x_{2}, y_{1}, y_{2}\right)=\frac{y_{1}^{2}+y_{2}^{2}}{2}+\frac{\alpha^{2} x_{1}^{2}}{2}-\frac{\nu^{2} x_{2}^{2}}{2}+\frac{b_{1} x_{1}^{4}}{4}+\frac{b_{2} x_{2}^{4}}{4}-R_{\epsilon}\left(x_{1}, x_{2}\right)
$$

Onde as seguintes condições são satisfeitas:

- $\alpha, \nu \neq 0$ e $b_{1}, b_{2}>0$

- A função $R_{\epsilon}=R_{\epsilon}\left(x_{1}, x_{2}\right)$ é analítica real, tendo a seguinte forma:

$$
R_{\epsilon}\left(x_{1}, x_{2}\right)=\epsilon\left(x_{1}^{2} x_{2}^{2}+x_{1}^{3} x_{2}^{3} \bar{R}\left(x_{1}, x_{2}\right)\right)
$$

Associado a esta função $H_{\epsilon}$, temos um campo vetorial $X_{H_{\epsilon}}$, chamado Campo Hamiltoniano, dado por:

$$
X_{H_{\epsilon}}\left(x_{1}, x_{2}, y_{1}, y_{2}\right)=\left(y_{1}, y_{2},-\alpha^{2} x_{1}-b_{1} x_{1}^{3}+\partial_{x_{1}} R_{\epsilon}, \nu^{2} x_{2}-b_{2} x_{2}^{3}+\partial_{x_{2}} R_{\epsilon}\right)
$$

Este campo vetorial dá origem a um fluxo no $\mathbb{R}^{4}$, indicado por $\phi_{t}^{H_{\epsilon}}$, e os conjuntos de nível, da função $H_{\epsilon}$, são chamados níveis de energia e eles são invariantes pelo fluxo $\phi_{t}^{H_{\epsilon}}$. A origem $(0,0,0,0)$ é uma singularidade do campo $X_{H_{\epsilon}}$, localizada no nível zero de energia. Os autovalores de $d X_{H_{\epsilon}}(0,0,0,0)$ 
são $\{\nu,-\nu, i \alpha,-i \alpha\}$. Portanto, a origem $(0,0,0,0)$ é uma singularidade do tipo sela-centro, localizada no nível zero de energia. Isso vale para qualquer valor do parâmetro $\epsilon$. Sistemas Hamiltonianos com um equilíbrio sela-centro é um tópico muito explorado na literatura. Alguns trabalhos importantes, sobre esse tema, são [18], [25], [24], [27] e [26].

O objetivo deste trabalho é estudar algumas propriedades da dinâmica dessa família de sistemas hamiltonianos, restrita a certos níveis de energia (níveis de energia que satisfazem algumas condições), quando o valor do parâmetro $\epsilon$ é pequeno. A análise será feita a partir de uma órbita periódica $\Gamma_{E}$, fixada em cada nível de energia $E$, segundo o Lema:

Lema 1.1.1 Seja $E>0$. Existe uma curva $\Gamma_{E}: \mathbb{R} \rightarrow \mathbb{R}^{4}$, com as seguintes propriedades:

1. $\Gamma_{E}$ é uma órbita periódica de $X_{H_{\epsilon}}$, com energia $E$ e período $T=T(E)$, para qualquer valor de parâmetro $\epsilon$.

2. A curva $\Gamma_{E}$ tem a seguinte forma: $\Gamma_{E}(t)=\left(0, x_{2}(t), 0, y_{2}(t)\right)$.

Esse lema será provado no capítulo 3. Para cada $E>0$, esta órbita $\Gamma_{E}$ é escolhida, por meio de uma análise do campo $X_{H_{\epsilon}}$ sobre o plano (invariante) $\left\{x_{1}=y_{1}=0\right\}$. É fundamental notar que a órbita $\Gamma_{E}$ de $X_{H_{\epsilon}}$ é a mesma (como uma curva no $\mathbb{R}^{4}$ ), tem o mesmo período e a mesma energia, para qualquer valor de parâmetro $\epsilon$. Em relação a esta órbita, temos:

Teorema 1.1.1 Sejam $E>0$ e $\Gamma_{E}$ a órbita dada pelo Lema 1.1.1. Suponhamos que o período $T=T(E)$ satisfaça a seguinte condição:

$$
\{\alpha T \neq 2 k \pi: k \in \mathbb{Z}\}
$$

Então existe $\epsilon_{0}=\epsilon_{0}(E)$, tal que se $|\epsilon|<\epsilon_{0}$, então valem as seguintes condições:

1. $\Gamma_{E}$ é elíptica no nível de energia $H_{\epsilon}^{-1}(E)$.

2. Existe uma órbita $\Gamma_{E}^{\prime}$ de $X_{H_{\epsilon}}$, com energia $E$, hiperbólica no nível de energia, tal que $\Gamma_{E}$ e $\Gamma_{E}^{\prime}$ estão linkadas em $H_{\epsilon}^{-1}(E)$.

O item 1 do Teorema 1.1.1 nos diz que se $\alpha T$ não for múltiplo inteiro de $2 \pi$, então a órbita $\Gamma_{E}$, de $X_{H_{\epsilon}}$, é elíptica no nível de energia, para $|\epsilon|$ pequeno. O estudo da Transformação de Poincaré dessa órbita de $X_{H_{\epsilon}}$, no nível de energia, pode ser reduzido ao estudo de um difeomorfismo, que 
preserva área, $\psi_{\epsilon}: \Omega_{\epsilon} \rightarrow \Omega_{\epsilon}$, onde $\Omega_{\epsilon} \subseteq \mathbb{R}^{2}$ é uma vizinhança de $(0,0)$ e este ponto é um ponto fixo elíptico.

Em relação ao item 2 do Teorema 1.1.1, ele afirma que existe uma órbita $\Gamma_{E}^{\prime}$, de $X_{H_{\epsilon}}$, com energia $E$, hiperbólica no nível de energia, tal que $\Gamma_{E}$ e $\Gamma_{E}^{\prime}$ formam um enlace de Hopf. A existência dessa órbita hiperbólica, na situação em que o nível de energia é pequeno e uma condição de convexidade no nível zero (o nível do equilíbrio sela-centro) é satisfeita, nos permite fazer uma possível conexão entre os resultados deste trabalho e os resultados de [12] e [13].

A condição do Teorema 1.1.1, em que $\alpha T$ não é múltiplo inteiro de $2 \pi$, indica que certos níveis de energia serão excluídos de nossa análise. De fato, teremos que excluir mais níveis de energia (impor mais restrições). Como foi dito acima, o estudo da Transformação de Poincaré da órbita $\Gamma_{E}$, de $X_{H_{\epsilon}}$, no nível de energia, pode ser reduzido ao estudo de um difeomorfismo, que preserva área, $\psi_{\epsilon}: \Omega_{\epsilon} \subseteq \mathbb{R}^{2} \rightarrow \Omega_{\epsilon}$, cuja origem é um ponto fixo elíptico. Impondo condições sobre os autovalores de $d \psi_{\epsilon}(0,0)$, é possível utilizar o Teorema da Forma Normal de Birkhoff (ver [14] e [23]), que nos garante que a expressão de $\psi_{\epsilon}$, em coordenadas polares $(\theta, r)$, tenha uma expressão bem definida, que depende de alguns coeficientes, chamados invariantes de Birkhoff. Na nossa análise, faremos uso de um desses invariantes, o chamado primeiro invariante de Birkhoff. É possível garantir que o Teorema da Forma Normal de Birkhoff possa ser aplicado ao estudo da Transformação de Poincaré de $\Gamma_{E}$, em $H_{\epsilon}^{-1}(E)$, excluindo-se mais alguns níveis de energia. Tendo em vista isso, definiremos o seguinte conjunto de energias (críticas):

$$
J \doteq\left\{E \in(0,+\infty): \alpha T \neq 2 k \pi, k \pi, \frac{k \pi}{2}, \frac{2 k \pi}{3}: k \in \mathbb{Z}\right\}
$$

Observemos que se $E \in J$, então a hipótese do Terema 1.1.1 é satisfeita. Em relação a uma vizinhança de $\Gamma_{E}$, no nível de energia, podemos afirmar:

Teorema 1.1.2 Existe uma função ${ }^{1} I_{B}: J \rightarrow \mathbb{R}$, tal que se $E \in J$ e $I_{B}(E) \neq 0$, então existe $\epsilon_{0}=\epsilon_{0}(E)$, tal que se $|\epsilon|<\epsilon_{0}$, então existem toros invariantes, pelo fluxo de $X_{H_{\epsilon}}$, com energia $E$, que se acumulam na órbita $\Gamma_{E}$.

Em relação a este Teorema, se $E \in J$, a órbita $\Gamma_{E}$, de $X_{H_{\epsilon}}$, é elíptica no nível de energia e o Teorema da Forma Normal de Birkhoff pode ser utilizado para a correspondente Transformação de Poincaré, $\psi_{\epsilon}: \Omega_{\epsilon} \rightarrow \Omega_{\epsilon}$, quando $|\epsilon|$ é pequeno. Em particular, quando $\epsilon=0$. O primeiro invariante de Birkhoff,

\footnotetext{
${ }^{1} I_{B}$ faz referência a invariante de Birkhoff. Assim, $I_{B}(E)$ representa o primeiro invariante de Birkhoff para o caso $\epsilon=0$.
} 
para $\epsilon=0$, pode ser expressado, nessas condições, como uma função da energia $E$. Definimos esse número real como sendo $I_{B}(E)$. Isso define a função $I_{B}=I_{B}(E)$. Se $I_{B}(E) \neq 0$, então, para $\epsilon$ suficientemente pequeno, o primeiro invariante de Birkhoff correspondente a $\psi_{\epsilon}: \Omega_{\epsilon} \rightarrow \Omega_{\epsilon}$, também, é não nulo. Portanto, a aplicação de Poincaré $\psi_{\epsilon}$, em coordenadas polares, satisfaz uma condição Twist (ver [14]); além disso, ela satisfaz as hipóteses do Teorema do Twist de Moser (ver [23]). Logo, existem curvas fechadas, invariantes por $\psi_{\epsilon}$, que se acumulam no ponto fixo elíptico $(0,0) \in \Omega_{\epsilon}$. Essas curvas fechadas e invariantes, quando vistas ao longo da órbita $\Gamma_{E}$, correspondem a toros invariantes, com energia $E$, que se acumulam em $\Gamma_{E}$. Veremos que no caso $\epsilon=0$, o domínio de $\psi_{0}: \Omega_{0} \rightarrow \Omega_{0}$ é inteiramente folheado por curvas fechadas e invariantes por $\psi_{0}$, cuja dinâmica de $\psi_{0}$, restrita a tais curvas fechadas, é conjugada a uma rotação. Portanto, os toros invariantes do Teorema 1.1.2 podem ser vistos, para o caso $\epsilon \neq 0$, pequeno, como toros sobreviventes do caso $\epsilon=0$. Poderíamos nos questionar se algum dos toros invariantes, para o caso $\epsilon=0$, é destruído pela perturbação. Nesse sentido, o próximo Teorema nos dá uma condição suficiente para que alguns desses toros invariantes sejam destruídos, pela perturbação.

Teorema 1.1.3 Sejam $E \in J$ e $\Gamma_{E}$ a órbita periódica do Lema 1.1.1. Suponhamos que as seguintes condições sejam satisfeitas:

1. $I_{B}(E) \neq 0$;

2. $\int_{0}^{T} x_{2}^{2}(s) \sin \{2 \alpha(T-s)\} d s \neq 0$;

Então existe $\epsilon_{0}=\epsilon_{0}(E)>0$, tal que se $|\epsilon|<\epsilon_{0}$, então existem zonas de instabilidade, em $H_{\epsilon}^{-1}(E)$, que se acumulam em $\Gamma_{E}$.

Nas hipóteses do Teorema 1.1.3, a órbita $\Gamma_{E}$ de $X_{H_{\epsilon}}$ é elíptica no nível de energia, para $|\epsilon|$ pequeno. A correspondente Transformação de Poincaré satisfaz uma condição twist. Na Teoria de Aplicações Twist, uma zona de instabilidade (ver [2]) é uma região anular invariante, cujo interior não contem curvas rotacionais invariantes. Nessas regiões invariantes, ocorrem alguns dos fenômenos mais interessantes relacionados a Teoria de Aplicações Twist, tais como existência de conjuntos de Aubry-Mather (não triviais) e entropia topológica positiva. Assim, as zonas de instabilidade, descritas no Teorema 1.1.3, são regiões invariantes entre toros invariantes (sem toros invariantes em seu interior), com dinâmica twist, se acumulando em $\Gamma_{E}$, dentro do nível de energia. Para provarmos este resultado de destruição de toros invariantes, fazemos uso de um critério perturbativo de destruição de curvas rotacionais 
invariantes para aplicações twist. Esse critério (assim como algumas de suas propriedades) está descrito em [28] e [29]. A hipótese 2, do Teorema 1.1.3, está relacionada a este critério.

Diante do resultado do Teorema 1.1.3, temos o seguinte corolário.

Corolário 1.1.1 Seja $E \in J$ e suponhamos que que as condições do Teorema 1.1 .3 sejam satisfeitas. Então, existe $\epsilon_{0}=\epsilon_{0}(E)>0$, tal que se $|\epsilon|<\epsilon_{0}$, com $\epsilon \neq 0$, então o fluxo de $X_{H_{\epsilon}}$, restrito a $H_{\epsilon}^{-1}(E)$, tem entropia topológica positiva.

Este resultado segue do Teorema 1.1 .3 e de um resultado de [1], que relaciona, para aplicações twist, curvas rotacionais invariantes e entropia topológica positiva.

Ao longo deste texto, destacaremos alguns níveis de energia que satisfazem as nossas hipóteses. De fato, por meio da influência do comportamento dinâmico próximo ao equilíbrio sela-centro $(0,0,0,0)$, localizado no nível zero de energia, mostraremos que "um conjunto grande de valores de energia", próximos ao nível zero, satisfazem as nossas hipóteses.

O texto está organizado da seguinte maneira. No capítulo 2, faremos uma revisão de alguns aspectos da Teoria de Sistemas Hamiltonianos que irão estar presentes ao longo deste texto.

No capítulo 3, iremos estudar algumas propriedades dinâmicas da família de sistemas hamiltonianos, definidos em (1.1). Neste capítulo, provaremos o Lema 1.1.1 e o Teorema 1.1.1. Além disso, definiremos a aplicação de Poincaré para estudo da dinâmica de $X_{H_{\epsilon}}$, próximo a órbita $\Gamma_{E}$, sobre o nível de energia. Desta forma, teremos uma família de aplicações de Poincaré associadas a órbita $\Gamma_{E}$. Colocaremos algumas questões, relacionadas a essa família de aplicações, que irão conduzir o andamento do restante do texto.

No capítulo 4, estudaremos uma mudança de coordenadas para o estudo das Transformações de Poincaré associadas a órbita $\Gamma_{E}$, via a Forma Normal de Birkhoff. Neste ponto, é colocada a importância de saber se algum invariante de Birkhoff é não nulo. No capítulo 5, estudaremos o primeiro invariante de Birkhoff, para essa família de aplicações. Por meio deste estudo, provaremos o Teorema 1.1.2 e concluiremos que, em certas condições e em coordenadas convenientes, as aplicações de Poincaré, associadas a órbita $\Gamma_{E}$, correspondem a aplicações twist simpléticas exatas. Com o objetivo de provar o Teorema 1.1.3, no capítulo 6 , apresentaremos um critério para a análise de destruição de curvas rotacionais invariantes para aplicações twist e o capítulo 7 trata de expressar este critério nas coordenas da seção de Poincaré construída. Parte dos cálculos, envolvidos nessa parte, estão no Apêndice A. É importante destacar que cada curva rotacional invariante, pela aplicação de 
Poincaré, corresponde a um toro invariante, pelo fluxo hamiltoniano, sobre o nível de energia.

No capítulo 8, analisaremos, com mais detalhes, o critério de destruição de curvas rotacionais invariantes, estabelecido nas coordenadas da seção, e provamos o Teorema 1.1.3 e o Corolário 1.1.1. O texto contém um segundo apêndice que trata da análise da interseção entre variedades estável e instável, associadas a uma subvariedade invariante normalmente hiperbólica, introduzida no capítulo 2. Este apêndice tem como objetivo dar uma complementar as informações dinâmicas da família de sistemas hamiltonianos, introduzido em (1.1), dadas no capítulo 2.

Em [4] e [5], os autores estudaram fluxos geodésicos em grupos de Lie não compactos. A análise, nestes trabalhos, se reduzem ao estudo de um sistema hamiltoniano com características próximas ao estudado neste texto. O estudo destes dois trabalhos motivou algumas questões que são tratadas no texto. 


\section{Capítulo 2}

\section{Teoria de Sistemas Hamiltonianos.}

O objetivo, deste capítulo, é introduzir alguns conceitos (nomenclaturas) e resultados relacionados a teoria de Sistemas Hamiltonianos, que irão aparecer ao longo desse texto, assim como tratar de alguns exemplos, que podem auxiliar na compreensão do resultado do texto. Os leitores mais familiarizados podem passar, diretamente, ao próximo capítulo.

\subsection{Sistemas Hamiltonianos}

Suponhamos $H: \mathbb{R}^{n} \longrightarrow \mathbb{R}$, função de classe $C^{2}$. Associamos, a $H$, o seguinte campo vetorial:

$$
\begin{aligned}
X_{H}: \mathbb{R}^{n} & \longrightarrow \mathbb{R}^{n} \\
\left(q_{1}, \ldots, q_{n}, p_{1}, \ldots, p_{n}\right) & \mapsto\left(\frac{\partial H}{\partial p_{1}}, \ldots, \frac{\partial H}{\partial p_{n}},-\frac{\partial H}{\partial q_{1}}, \ldots,-\frac{\partial H}{\partial q_{n}}\right)
\end{aligned}
$$

Este campo é uma campo vetorial de classe $C^{1}$, chamado campo hamiltoniano associado a função $H$, sendo esta função chamada de função hamiltoniana. A equação de Hamilton associada à função $H$ consiste da seguinte equação diferencial ordinária:

$$
\left\{\begin{array}{l}
\dot{q}=\frac{\partial H(q, p)}{\partial p}, \\
\dot{p}=-\frac{\partial H(q, p)}{\partial q}
\end{array}\right.
$$


Portanto, uma solução da equação de Hamilton associada a $H$ consiste em uma trajetória do campo Hamiltoniano $X_{H}$. Poderíamos, da mesma forma, considerar funções $H$ dependendo do tempo. Ou seja, a função $H$ dependeria de uma variável real a mais $t, H=H(q, p, t)$. Nesse caso, o campo Hamiltoniano seria não-autônomo. Para o caso autônomo, a função $H$ é uma integral primeira do campo hamiltoniano $X_{H}$. Assim, os conjuntos de nível da função $H$, que são chamados níveis de energia do sistema, são invariantes pelo fluxo do campo $X_{H}$.

A Teoria de Sistemas Hamiltonianos surgiu como uma formulação alternativa para as Leis de Newton da Mecânica Clássica. Para ilustração disso, consideremos o movimento de uma partícula de massa $m$, no espaço $\mathbb{R}^{n}$, de coordenadas $\left(q_{1}, \ldots, q_{n}\right)$, submetida à ação de uma força conservativa $F$. Nesse caso, existe uma função diferenciável $V: \mathbb{R}^{n} \longrightarrow \mathbb{R}$, talque:

$$
F(q)=-\nabla V(q)
$$

A função $V$ é chamada energia potencial do sistema. A energia cinética é dada por:

$$
E_{\text {cin }}=\sum_{i=1}^{n} \frac{m_{i} \dot{q}_{i}^{2}}{2}
$$

A energia total é dada por $E_{T}=E_{c i n}+V$ e é uma quantidade conservada pelo sistema, ou seja, é uma integral primeira associada a equação de segunda ordem, que está associada à Lei de Newton:

$$
\ddot{q}(t)=-\frac{1}{m} \nabla V(q(t))
$$

O momento linear da partícula é definido como: $p=m \dot{q}$. Escrevendo a função energia nas variáveis posição e momento, obtemos:

$$
H(q, p)=\sum_{i=1}^{n} \frac{p_{i}^{2}}{2 m}+V(q)
$$

Então, a equação de Hamilton, associada à função $H=H(q, p)$, é dada por:

$$
\left\{\begin{array}{l}
\dot{q}_{i}=\frac{p_{i}}{m}, \\
\dot{p}_{i}=-\frac{\partial V}{\partial q_{i}}(q) .
\end{array}\right.
$$


Observemos que esta equação é equivalente à equação (1), diante da relação: $p=m \dot{q}$. De um modo geral, é mais conveniente tratar equações diferenciais de primeira ordem a equações diferenciais de segunda ordem.

Em Sistemas Hamiltonianos, o espaço $\mathbb{R}^{2 n}$, das variáveis $(q, p)$ é chamado espaço de fase do sistema. O espaço $\mathbb{R}^{n}$ das variáveis $q$ é chamado espaço de configuração dos sistema.

Exemplo 2.1.1 Hamiltoniano associado ao fluxo geodésico de uma métrica hiperbólica no semiplano $y>0$ :

$$
H\left(x, y, p_{x}, p_{y}\right)=\frac{y^{2}}{4}\left(p_{x}^{2}+p_{y}^{2}\right) .
$$

Este exemplo está contido em [19], que contém muitos outros exemplos interessantes. Outros exemplos podem ser encontrados em [3].

Exemplo 2.1.2 Consideremos a Hamiltoniana $H: \mathbb{R}^{2} \rightarrow \mathbb{R}$, dada por:

$$
H(x, y)=\frac{y^{2}}{2}-\cos x .
$$

Temos que a equação de Hamilton associada é dada por:

$$
\left\{\begin{array}{l}
x^{\prime}=y, \\
y^{\prime}=-\sin x .
\end{array}\right.
$$

$O$ retrato de fase do campo Hamiltoniano $X_{H}(x, y)=(y,-\sin x)$ é esboçado na Figura 1.

Os níveis de energia de $H$ corresponde aos conjuntos $S_{E}=\{(x, y)$ : $y= \pm \sqrt{2(E+\cos x)}\}$. Portanto, para $E \geqslant 1, S_{E}$ será a união de dois gráficos invariantes. Devemos notar que o campo $X_{H}$ possui as seguintes singularidades:

$$
\left\{Q_{n}=(2 \pi n, 0): n \in \mathbb{Z}\right\} \text { e }\left\{P_{n}=((2 n+1) \pi, 0): n \in \mathbb{Z}\right\}
$$

Os pontos $Q_{n}$ são elípticos, enquanto os pontos $P_{n}$ são hiperbólicos. Os pontos hiperbólicos estão no nível de energia $H=1$ e vale a seguinte relação entre as variedades invariantes de singularidades hiperbólicas de $X_{H}$ :

$$
W^{s}\left(P_{n}\right)=W^{u}\left(P_{n-1}\right), \forall n \in \mathbb{Z}
$$

Exemplo 2.1.3 Consideremos, agora, a seguinte Hamiltoniana, dependente do tempo e de um parâmetro $\epsilon, H_{\epsilon}: \mathbb{R}^{2} \times \mathbb{R} \rightarrow \mathbb{R}$, dada por: 


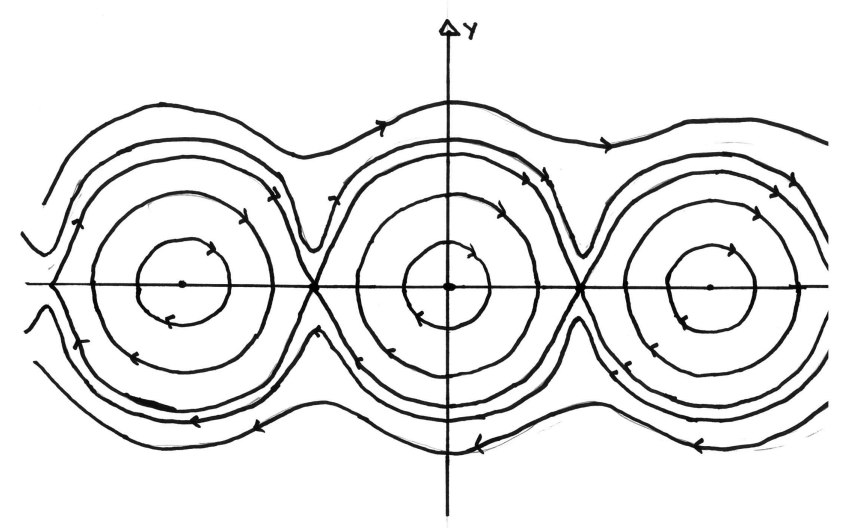

Figura 2.1: Retrato de Fase da equação diferencial do pêndulo.

$$
H_{\epsilon}((x, y), t)=\frac{y^{2}}{2}-\cos x+\epsilon x \sin \omega t
$$

Nesse caso, a equação de Hamilton associada é dada por:

$$
\left\{\begin{array}{l}
x^{\prime}=y, \\
y^{\prime}=-\sin x-\epsilon \sin \omega t .
\end{array}\right.
$$

Podemos analisar o campo Hamiltoniano $X_{H_{\epsilon}}$ como uma perturbação do campo Hamiltoniano do exemplo anterior, do pendulo, sendo o termo perturbativo dependente periodicamente do tempo. Em casos assim, em que a hamiltoniana é dependente do tempo, ela não será mais uma integral primeira da equação de Hamilton.

É possivel mostrar que para $\epsilon$ suficientemente pequeno, para todo $n \in \mathbb{Z}$, teremos uma singularidade do campo $P_{n}(\epsilon)$, dependendo diferenciavelmente do parâmetro $\epsilon$, tal que $P_{n}(0)$ coincide com as singularidades hiperbólicas do exemplo anterior. Tínhamos $W^{s}\left(P_{n}(0)\right)=W^{u}\left(P_{n+1}(0)\right)$. Porém, em [35], mostra-se que para $\epsilon \neq 0$, pequeno, as variedades invariantes deixarão de coincidir. Além disso, elas passam a se interceptarem transversalmente, ou seja, $W^{s}\left(P_{n}(\epsilon)\right) \pitchfork W^{u}\left(P_{n+1}(\epsilon)\right) \neq \emptyset$. Este sistema passa, então, a ter um comportamento caótico, conforme discutido em [35].

O próximo exemplo apresenta alguns resultados do sistema hamiltoniano analisado em [5] e [4]. Conforme comentado na seção 1 deste texto, este exemplo motivou algumas das questões deste trabalho. 
Exemplo 2.1.4 (Hamiltoniana de [4] e [5].) Os trabalhos [5] e [4] tratam do estudo de fluxos geodésicos em grupos de Lie não compactos, associados a métricas invariantes a esquerda. Em particular, considera-se o grupo de Lie $T_{4}$, das matrizes de ordem 4 e triangulares superiores, com o elemento 1 na diagonal principal. Em [5], há uma exposição sobre como a dinâmica de certos fluxos geodésicos, invariantes a esquerda, podem ser estudados por meio de uma sistema hamiltoniano no $\mathbb{R}^{4}$, via um processo de redução simplética. O sistema hamiltoniano obtido, neste processo, é dado pela seguinte hamiltoniana:

$$
2 H=x^{2}+\left(X^{2}-\frac{1}{2}\right)^{2}+y^{2}+\alpha^{2} Y^{2}+y^{4}-2 X^{2} Y^{2}
$$

Estamos utilizando as notações de [4] e [5], onde trabalha-se com coordenadas $(X, Y, x, y) \in \mathbb{R}^{4}$. Assim, a Hamiltoniana pode ser reescrita como:

$$
H=\frac{x^{2}+y^{2}}{2}+\frac{\alpha^{2} Y^{2}}{2}+\frac{Y^{4}}{2}-\frac{X^{2}}{2}+\frac{X^{4}}{2}-X^{2} Y^{2}
$$

Em [4] e [5], mostra-se a existência de um horseshoe para essa familia de sistemas hamiltonianos (a análise depende do parâmetro $\alpha$ ). Apresentaremos, a seguir, as principais etapas neste processo. Seguiremos por partes.

- Dado $\epsilon>0$, consideremos a família de aplicações $h_{\epsilon}: \mathbb{R}^{4} \rightarrow \mathbb{R}^{4}$, definidas por:

$$
h_{\epsilon}(X, Y, x, y)=\left(X, \frac{Y}{\sqrt{\epsilon}}, x, \frac{y}{\sqrt{\epsilon}}\right)
$$

Segue que $h_{\epsilon}$ conjuga o fluxo do campo $X_{H}$ ao fluxo do campo $F_{\epsilon}$, dado por:

$$
F_{\epsilon}(X, Y, x, y)=\left(x, y, X-2 X^{3}+2 \epsilon X Y^{2},-\alpha^{2} Y+2 Y X^{2}-2 \epsilon Y^{3}\right)
$$

Devemos notar que, apesar de a transformação $h_{\epsilon}$ não estar definida para $\epsilon=0$, o campo $F_{\epsilon}$ está bem definido para $\epsilon=0$ e é dado por:

$$
F_{0}(X, Y, x, y)=\left(x, y, X-2 X^{3},-\alpha^{2} Y+2 Y X^{2}\right)
$$

- Analisemos algumas propriedades do campo $F_{0}$. Consideremos a seguinte subvariedade:

$$
S=\left\{(X, Y, x, y) \in \mathbb{R}^{4}: X=x=0\right\}
$$


Esta é uma subvariedade invariante pelo fluxo do campo $F_{0}$. Além disso, $S$ é normalmente hiperbólica para o fluxo de $F_{0}$. Dessa forma, vamos determinar as variedades estável e instável (indicadas, respectivamente, por $W_{0}^{+}(S)$ e $W_{0}^{-}(S)$ ). Consideremos a função $h: \mathbb{R}^{4} \rightarrow \mathbb{R}^{4}$, dada por:

$$
h(X, Y, x, y)=x^{2}+\left(X^{2}-\frac{1}{2}\right)^{2}
$$

Essa função h é uma integral primeira do campo $F_{0}$. Além disso, por meio dessa função e analisando o comportamento do campo nas direções $(X, x)$, é possível verificar que:

$$
W_{0}^{+}(S)=W_{0}^{-}(S)=h^{-1}\left(\frac{1}{4}\right)
$$

- A subvariedade $S$ é, ainda, invariante pelo fluxo do campo $F_{\epsilon}$, para qualquer $\epsilon>0$. Além disso, $S$ é normalmente hiperbólica para o fluxo de $F_{\epsilon}$, para qualquer $\epsilon$.

- Sobre o conjunto $S \backslash W_{0}^{ \pm}(S)$, o fluxo de $F_{0}$ é dado por:

$$
\left\{\begin{array}{l}
X(t)= \pm \operatorname{sech}\left(t+t_{0}\right), x(t)=\mp \operatorname{tangh}\left(t+t_{0}\right) \operatorname{sech}\left(t+t_{0}\right) \\
Y(t)=c_{0} Y_{0}\left(t+t_{0}\right) c_{1} Y_{1}\left(t+t_{0}\right), y=\dot{Y}
\end{array}\right.
$$

Onde $\left\{Y_{0}, Y_{1}\right\}$ é uma base do espaço de soluções da seguinte equação diferencial ordinária:

$$
Y^{\prime \prime}+\left\{\alpha^{2}-2 \operatorname{sech}^{2}(t)\right\} Y
$$

Se $Y(0)=Y_{0, j}$ e $\dot{Y}(0)=\dot{Y}_{0, j}$, exige-se:

$$
W=Y_{0,0} \dot{Y}_{0,1}-Y_{0,1} \dot{Y}_{0,0} \neq 0
$$

- Para $\epsilon \neq 0$, suficientemente pequeno, temos as subvariedades estável e instável, relacionadas a $S, W_{\epsilon}^{+}(S)$ e $W_{\epsilon}^{-}(S)$. A análise da interseção entre essas variedades invariantes pode ser feita por meio da função de Melnikov, que mede a "€-separação" entre elas, podendo nos dar informação sobre interseção transversal entre essas variedades. A função de Melnikov pode ser vista como uma função definida sobre $W_{0}^{ \pm}(S) \backslash S$. Para caracterizar este conjunto, utilizamos as igualdades (2.2) e (2.3), $e$, assim, épossivel trabalhar com coordenadas $\left(t_{0}, c_{0}, c_{1}\right)$. Dessa forma, a função de Melnkov pode ser escrita como: 


$$
m\left(t_{0}, c_{0}, c_{1}\right)=m_{00} c_{0}^{2}+2 m_{01} c_{0} c_{1}+m_{11} c_{1}^{2}
$$

Onde: $m_{i j}=\int_{\mathbb{R}} \dot{q}(s) Y_{i}(s) Y_{j}(s) d s$ e $q(s)=2 \operatorname{sech}^{2}(s)$. Observemos que essa representação, da função de Melnikov, depende da escolha de uma base $\left\{Y_{0}, Y_{1}\right\}$ para o espaço de soluções da equação (2.3).

Portanto, nessas coordenadas, a função de Melnikov é uma forma quadrática.

Pontos onde ocorrem interseção transversal entre $W_{\epsilon}^{+}(S)$ e $W_{\epsilon}^{-}(S)$ estão relacionados a zeros não degenerados da função $m=m\left(t_{0}, c_{0}, c_{1}\right)$.

- Em [5], por meio da escolha da seguinte base, para o espaço de soluções de (2.3), $Y_{j}(0)=1-j$ e $\dot{Y}_{j}(0)=j$, mostra-se que se $\alpha \neq 1$, então há zeros não degenerados da função de Melnikov. Ou seja, há interseção transversal entre $W_{\epsilon}^{+}(S)$ e $W_{\epsilon}^{-}(S)$, para $\epsilon$ suficientemente pequeno.

Em [4], "por meio de uma escolha mais específica"de base $\left\{Y_{0}, Y_{1}\right\}$, mostra-se que vale a mesma conclusão para qualquer $\alpha \in \mathbb{R}$.

Portanto, o fluxo do campo $F_{\epsilon}$ tem um horseshoe e o mesmo vale para o campo hamiltoniano $X_{H}$. Assim, o fluxo de $X_{H}$ tem entropia topológica positiva.

Sistemas Hamiltonianos podem ser definidos em espaços mais gerais que os espaços euclidianos $\mathbb{R}^{2 n}$. Observemos que o campo Hamiltoniano $X_{H}$, associado à função $H: \mathbb{R}^{2 n} \longrightarrow \mathbb{R}$, pode ser obtido do campo gradiente de $H, \nabla H$, por meio de:

$$
X_{H}=J_{0} \nabla H, \text { onde: } J_{0}=\left(\begin{array}{cc}
0_{n} & \mathrm{Id} \\
-\mathrm{Id} & 0_{n}
\end{array}\right) .
$$

Observemos a relação direta entre o campo $X_{H}$ e a função $H$.

$$
d_{x} H(v)=\langle\nabla H(x) ; u\rangle=\left\langle-J_{0} X_{H}(x), v\right\rangle=\left\langle X_{H}(x) ; J_{0} v\right\rangle
$$

Diante disso, definimos a seguinte aplicação bilinear em $\mathbb{R}^{2 n}$ :

$$
\begin{gathered}
\Omega: \mathbb{R}^{2 n} \times \mathbb{R}^{2 n} \longrightarrow \mathbb{R} \\
(u, v) \longmapsto\left\langle u ; J_{0} v\right\rangle
\end{gathered}
$$

Segue que:

$$
d H=i_{X_{H}} \Omega
$$

Essa relação determina o campo $X_{H}$ por meio da aplicação bilinear $\Omega$. Essa análise nos permite estender a noção de sistemas hamiltonianos para espaços mais gerais: as variedades simpléticas. 


\subsection{Geometria Simplética}

Algumas propriedades, relacionadas a Sistemas Hamiltonianos, tais como autovalores de uma aplicação de Poincare de uma órbita periódica, podem ser melhor compreendidas num "contexto simplético". Uma ótima introdução a geometria simplética pode ser encontrada em [31]. Uma variedade simplética consiste de uma variedade diferenciável, na qual o espaço tangente em todo ponto possui uma estrutura de espaço vetorial simplético que varia diferenciavelmente com o ponto. Sendo assim, definiremos, inicialmente, um espaço vetorial simplético.

Definição 2.2.1 Um espaço vetorial simplético é um par $\left(V, \Omega_{0}\right)$, onde $V$ é um espaço vetorial real de dimensão $m$ e $\Omega_{0}: V \times V \longrightarrow \mathbb{R}$ é uma aplicação bilinear e anti-simétrica que satisfaz:

$$
\Omega_{0}^{*}: V \longrightarrow V^{*}
$$

Definida por $\Omega_{0}^{*}(v)=\Omega_{0}\left(v,,^{\circ}\right)$, é não degenerada, ou seja, é um isomorfismo.

Espaços vetoriais simplético possuem dimensão par. Para ser mais preciso, se $\left(V, \Omega_{0}\right)$ é um espaço vetorial simplético, então existe uma base de $V$, $\left\{e_{1}, \ldots, e_{n}, f_{1}, \ldots, f_{n}\right\}$, satisfazendo a seguinte condição:

$$
\Omega_{0}\left(e_{i}, f_{j}\right)=\delta_{i j} \text { e } \Omega_{0}\left(e_{i}, e_{j}\right)=0=\Omega_{0}\left(f_{i}, f_{j}\right), \forall i, j .
$$

Em tal base, a aplicação $\Omega_{0}$ se escreve da seguinte forma:

$$
\Omega_{0}(u, v)=\left\langle u, J_{0} v\right\rangle \text {, onde, } J_{0}=\left(\begin{array}{cc}
0_{n} & \mathrm{Id} \\
-\mathrm{Id} & 0_{n}
\end{array}\right) .
$$

Se $\left(V_{1}, \Omega_{1}\right)$ e $\left(V_{2}, \Omega_{2}\right)$ são espaços vetoriais simpléticos, então um simplectomorfismo linear, entre eles, consiste de um isomorfismo linear satisfazendo: $T^{*} \Omega_{2}=\Omega_{1}$. A existência da base acima implica que todo espaço vetorial simplético é simplectomorfo a $\left(\mathbb{R}^{2 n}, \Omega_{0}\right)$. O conjunto dos simplectomorfismos linear de um espaço vetorial simplético pode ser indentificado com:

$$
\operatorname{Sp}(n)=\left\{A \in G L(2 n, \mathbb{R}), A J_{0} A=J_{0}\right\}
$$

Trata-se de um grupo com a operação de multiplicação de matrizes. Mais a frente, quando falarmos de autovalores da aplicação de Poincaré de uma órbita periódica de um sistema Hamiltoniano, voltaremos a destacar algumas propriedades deste grupo. 
É importante destacar alguns tipos de subespaços vetoriais de um espaço vetorial simplético. Ao contrário de um produto interno, que quando restrito a um subespaço vetorial continua sendo um produto interno, a forma simplética pode deixar de ser uma forma simplética.

Sejam $(V, \Omega)$ um espaço vetorial simplético e $W \subseteq V$ um subespaço vetorial. Associado ao subespaço $W$, definimos o seguinte outro subespaço, chamado ortogonal simplético de $W$ :

$$
W^{\perp}=\{v \in V: \Omega(v, u)=0, \forall u \in W\}
$$

Assim, dizemos que:

- $W$ é isotrópico, se $W \subseteq W^{\perp}$;

- $W$ é coisotrópico, se $W^{\perp} \subseteq W$;

- $W$ é isotrópico, se $W=W^{\perp}$;

Não é difícil verificar que vale $\left(W^{\perp}\right)^{\perp}=W$ e também vale a seguinte relação:

$$
\operatorname{dim} V=\operatorname{dim} W+\operatorname{dim} W^{\perp}
$$

Diante dessa pequena introdução aos espaços vetoriais simpléticos, passemos às variedades simpléticas.

Definição 2.2.2 Uma variedade simplética é um par $(M, \omega)$, onde $M$ é uma variedade simplética e $\omega \in \Omega^{2}(M)$ é uma 2-forma fechada e não-degenerada.

Observemos que se $(M, \omega)$ é uma variedade simplética, então $\left(T_{p} M, \omega_{p}\right)$ é um espaço vetorial simplético para todo $p \in M$. Logo, a dimensão da variedade $M$ tem de ser par. Além disso, $M$ tem que ser orientável, pois $\omega^{n}$ define uma forma de volume sobre $M$.

Exemplo 2.2.1 $\left(\mathbb{R}^{2 n}, \omega_{0}\right)$ é uma variedade simplética, onde utilizando coordenadas $\left(q_{1}, \ldots, q_{n}, p_{1}, \ldots, p_{n}\right)$ para $\mathbb{R}^{2 n}, \omega_{0}$ é dado por:

$$
\omega_{0}=\sum_{i=1}^{n} d q_{i} \wedge d p_{i}
$$

Exemplo 2.2.2 Consideremos a 2-esfera $\mathbb{S}^{2} \subseteq \mathbb{R}^{3}$. Definimos:

$$
\omega_{p}(u, v)=\langle p ; u \times v\rangle,
$$


onde, $u, v \in T_{p} \mathbb{S}^{2}, p \in \mathbb{S}^{2}$. $\left(\mathbb{S}^{2}, \omega\right)$ é uma variedade simplética.

É importante destacar que a única esfera que admite uma estrutura simplética é a esfera de dimensão 2. Há restrições de natureza topológica (além da dimensão). Ver [21] para maiores detalhes.

Exemplo 2.2.3 (Fibrados Cotangentes)

Seja $M^{n}$ uma variedade e consideremos o seu fibrado cotangente $\pi: T^{*} M \longrightarrow$ $M$. Consideremos a 1-forma em $T^{*} M$ :

$$
\alpha_{(x, p)}(v)=p\left(d_{(x, p)} \pi(v)\right)
$$

Essa 1-forma é chamada 1-forma de Liouville. A 2-forma:

$$
\omega=-d \alpha
$$

define uma estrutura simplética em $T^{*} M$.

Localmente em $T^{*} M$, com coordenadas $\left(q_{1}, \ldots, q_{n}, p_{1}, \ldots, p_{n}\right)$, teremos:

$$
\alpha=\sum_{i=1}^{n} p_{i} d x_{i} \text { e } \omega_{0}=\sum_{i=1}^{n} d q_{i} \wedge d p_{i}
$$

Um fato importante sobre as variedades simpléticas é que, se duas delas possuem a mesma dimensão, então elas são, localmente, equivalentes. Para formalizar isso, vamos definir essa noção de equivalência.

Definição 2.2.3 Sejam $\left(M_{1}, \omega_{1}\right)$ e $\left(M_{2}, \omega_{2}\right)$ variedades simpléticas. Dizemos que um difeomorfismo $\varphi: M_{1} \longrightarrow M_{2}$ é um simplectomorfismo se $\varphi^{*} \omega_{2}=\omega_{1}$.

Observemos que se $\varphi: M_{1} \longrightarrow M_{2}$ é um simplectomorfismo, então sua diferencial $d_{p} \varphi: T_{p} M_{1} \longrightarrow T_{\varphi(p)} M_{2}$ é um simplectomorfismo linear.

O próximo teorema, chamado Teorema de Darboux, nos diz que quaisquer duas variedades simpléticas, com mesma dimensão, são localmente simplectomorfas.

Teorema 2.2.1 Sejam $\left(M^{2 n}, \omega\right)$ variedade simplética e $p \in M$. Existem $V_{p}$, vizinhança de $p$ em $M, V_{0}$, vizinhança da origem em $\mathbb{R}^{2 n}$ e um difeomorfismo $\varphi: V_{0} \longrightarrow V_{p}$ tal que:

$$
\varphi^{*} \omega=\omega_{0} \text {, onde } \omega_{0} \text { é a forma canônica. }
$$

Diante de uma estrutura simplética em uma variedade, podemos falar em sistemas Hamiltonianos na variedade. A definição é muito natural, tendo em vista a equação (2). 
Definição 2.2.4 Sejam $\left(M^{2 n}, \omega\right)$ variedade simplética e $H: M \longrightarrow \mathbb{R}$ uma função diferenciável. O Campo Hamiltoniano associado a função $H$, denotado por $X_{H}$, é definido pela seguinte relação:

$$
d H=i_{X_{H}} \omega .
$$

Observação 2.2.1 Observemos que, como $\omega$ é não-degenerada, existe e é único um campo vetorial definido por essa igualdade. O fato de $\omega$ ser fechada nos garante que o fluxo, associado ao campo $X_{H}$, é um simplectomorfismo em todos os instantes.

Se consideramos a variedade simplética $\left(\mathbb{R}^{2 n}, \omega_{0}\right)$, a definição acima coincide com a definição de campo Hamiltoniano dada no início da subseção anterior. Quando dizemos uma função Hamiltoniana definida num espaço $\mathbb{R}^{2 n}$, está implícito que a estrutura simplética é a canônica.

Vamos a alguns exemplos de Sistemas Hamiltonianos em variedades.

Exemplo 2.2.4 Sejam $\left(M^{n}, g\right)$ uma variedade riemanniana e $\pi: T^{*} M \longrightarrow$ $M$ seu fibrado cotangente com sua estrutura simplética ususal. Definimos:

$$
\begin{gathered}
H: T^{*} M \longrightarrow \mathbb{R} \\
(x, p) \longmapsto \frac{\|p\|_{x}}{2}
\end{gathered}
$$

Onde $\|p\|_{x}$ é a norma em $T_{x}^{*} M$, induzida por $g_{x}$. O sistema Hamiltoniano associado a $H$ representa o fluxo geodésico associado a métrica $g$. A projeção das trajetórias de $X_{H}$ em $M$ correspondem às geodésicas da métrica $g$.

Exemplo 2.2.5 Consideremos $\left(M^{n}, g\right)$ uma variedade riemanniana, $V$ : $M \longrightarrow \mathbb{R}$ uma função diferenciável e $\pi: T^{*} M \longrightarrow M$ o fibrado cotangente com a estrutura simplética usual. Definimos:

$$
\begin{aligned}
H & : T^{*} M \longrightarrow \mathbb{R} \\
(x, p) & \longmapsto \frac{\|p\|_{x}}{2}+V(x)
\end{aligned}
$$

Esse tipo de sistema representa uma generalização de Hamiltonianos da Mecânica Clássica.

Destacaremos alguns tipos de subvariedades de uma variedade simplética. Ao contrário do que ocorre em geometria riemanniana, onde uma métrica no espaço ambiente induz uma estrutura riemanniana na subvariedade, tornandoa riemanniana, uma subvariedade associada a uma variedade simplética pode não ser simplética (por exemplo, uma subvariedade de dimensão ímpar). Nesse sentido, é conveniente estabelecer algumas situações.

Supondo $\left(M^{2 n}, \omega\right)$ variedade simplética e $N \subseteq M$ uma subvariedade, seja $i: N \hookrightarrow M$ a inclusão.

Dizemos que: 
- $N$ é isotrópica, se:

$$
\forall p \in N, T_{p} N \text { é subespaço isotrópico de }\left(T_{p} M, \omega_{p}\right) \text {. }
$$

Nesse caso, $\operatorname{dim} N \leq \operatorname{dim} M$

- $N$ é coisotrópica, se

$$
\forall p \in N, T_{p} N \text { é subespaço coisotrópico de }\left(T_{p} M, \omega_{p}\right)
$$

Nesse caso, $\operatorname{dim} N \geq \operatorname{dim} M$

- $N$ é Lagrangiana, se:

$$
\forall p \in N, T_{p} N \text { é subespaço Lagrangiano de }\left(T_{p} M, \omega_{p}\right)
$$

Nesse caso, $\operatorname{dim} N=n$. Uma outra forma de expressar essa condição, seria dizendo que $i^{*} \omega=0$.

- $N$ é simplética, se:

$$
\forall p \in N, T_{p} N \text { é subespaço simplético de }\left(T_{p} M, \omega_{p}\right)
$$

Nesse caso, $\operatorname{dim} N$ é um número par. Outra forma de expressar essa condição, seria dizendo que $i^{*} \omega$ é uma forma simplética sobre $N$.

A seguir, dois exemplos importantes:

Exemplo 2.2.6 Sejam $\left(M^{2 n}, \omega\right)$ variedade simplética e $H: M \longrightarrow \mathbb{R}$ uma função diferenciável. Suponhamos $c \in \mathbb{R}$ valor regular de $H$. Segue que o nivel de energia $\Sigma_{c}=H^{-1}(c)$ é uma hiperfície de $M$ tal que:

$$
\forall p \in M, T_{p} \Sigma_{c}=\operatorname{Ker}\left(d_{p} H\right)
$$

Observemos que $X_{H}$ é tangente a $\Sigma_{c}$, pois $\Sigma_{c}$ é invariante pelo fluxo de $X_{H}$. Seja $S \subseteq \Sigma_{c}$ subvariedade de codimensão 2 em $M$, que é transversal ao campo $X_{H}$ em $\Sigma_{c}$. Então, S é uma subvariedade simplética de $(M, \omega)$. Ver [35], para maiores detalhes.

Exemplo 2.2.7 Consideremos $\left(T^{*} M, \omega\right)$ o fibrado cotangente de uma variedade diferenciável $M$, munido da estrutura simplética usual. Seja $\eta \in$ $\Omega^{1}(M)$. Associado a esta 1-forma $\eta$, temos uma subvariedade em $T^{*} M$, que corresponde ao gráfico de $\eta$ : 


$$
\Gamma_{\eta}=\left\{\left(x, \eta_{x}\right) \in T^{*} M: x \in M\right\}
$$

Temos que $\Gamma_{\eta}$ é subvariedade Lagrangiana de $\left(T^{*} M, \omega\right)$ se, e somente se, $d \eta=0$.

Pensemos no caso em que $\eta$ é exata. Assim, $\exists u: M \longrightarrow \mathbb{R}$ diferenciável tal que $\eta=d u$. Seja $H: T^{*} M \longrightarrow \mathbb{R}$ diferenciável. A subvariedade $\Gamma_{\eta} e ́$ invariante pelo fluxo do campo $X_{H}$ se, e somente se:

$$
H\left(x, d_{x} u\right)=\text { constante, } \forall x \in M
$$

Ou seja, u é solução da equação de Hamilton-Jacobi associada a H. Concluímos que subvariedades Lagrangiana exatas, que são invariantes pelo fluxo de um sistema Hamiltoniano, correspondem a uma solução da equação de Hamilton Jacobi do sistema.

\subsection{Análise de Órbitas Periódicas}

O estudo local do comportamento das trajetórias de um campo, próximos a uma órbita periódica, pode ser feito por meio de uma análise da transformação de Poincaré associada à órbita ${ }^{1}$. É importante analisar os autovalores da diferencial da transformação de Poincaré no correspondente ponto da órbita. Como a transformação de Poincaré pode ser escrita em termos do fluxo, existe uma relação entre os autovalores da diferencial da aplicação de Poincaré e da diferencial do fluxo. Vamos analisar um pouco essa situação, para o caso de Sistemas Hamiltonianos.

Sejam $\left(M^{2 n}, \omega\right)$ uma variedade simplética e $H: M \longrightarrow \mathbb{R}$ uma função diferenciável. Suponhamos $\varphi_{t}$ o fluxo do campo Hamiltoniano associado a $H, X_{H}$, e $p \in M$ um ponto periódico de $X_{H}$, com período $T>0$. Assim, conforme discutido na seção anterior, $\varphi_{T}: M \longrightarrow M$ é um simplectomorfismo e, sua diferencial, $d_{p} \varphi_{T}: T_{p} M \longrightarrow T_{p} M$ é um simplectomorfismo linear. É possível descrever algumas propriedades do espectro de simplectomorfismos lineares. Faremos essa análise num contexto mais geral.

Seja $\left(V^{2 n}, \Omega\right)$ um espaço vetorial simplético. Fixada uma base simplética para $(V, \Omega)$, como foi estabelecido acima, podemos identificar o conjunto dos simplectomorfismos lineares de $(V, \Omega)$ com o seguinte subconjunto de matrizes:

$$
S p(n)=\left\{A \in G L(2 n, \mathbb{R}): A^{T} J_{0} A=J_{0}\right\},
$$

\footnotetext{
${ }^{1} \operatorname{Ver}([33])$.
} 
onde a matriz $J_{0}$ é dada por:

$$
J_{0}=\left(\begin{array}{cc}
0_{n} & \mathrm{Id} \\
-\mathrm{Id} & 0_{n}
\end{array}\right) .
$$

Naturalmente, $S p(n)$ é um grupo com respeito a multiplicação matricial e se $A \in S p(n)$, então $\operatorname{det} A=1$.

A próxima proposição nos dá uma propriedade muito importante do espectro dessas matrizes.

Proposição 2.3.1 Sejam $A \in S p(n)$ e $\sigma(A)$ o seu espectro. Se $\lambda \in \sigma(A)$, então $\lambda^{-1}, \bar{\lambda}, \bar{\lambda}^{-1} \in \sigma(A)$. Além disso, $\lambda, \lambda^{-1}, \bar{\lambda}, \bar{\lambda}^{-1}$ possuem, todos, a mesma multiplicidade algébrica.

Portanto, os autovalores da diferencial do fluxo:

$$
d_{p} \varphi_{T}: T_{p} M \longrightarrow T_{p} M,
$$

satisfazem essa propriedade. Observemos, ainda, que $X_{H}(p)$ é autovetor de $d_{p} \varphi_{T}$, associado ao autovalor 1 . De fato, pela propriedade de fluxo, temos:

$$
\varphi_{s} \circ \varphi_{t}(p)=\varphi_{t} \circ \varphi_{s}(p), \forall s, t \in \mathbb{R} .
$$

Derivando com respeito a $s$, obtemos:

$$
d_{\varphi_{s}(p)} \varphi_{t}=X_{H}\left(\varphi_{s+t}(p)\right)
$$

Fazendo $s=0$ e $t=T$, conseguimos que:

$$
d_{p} \varphi_{T}\left(X_{H}(p)\right)=X_{H}(p) .
$$

Segue então a afirmação feita.

Seja $\Sigma$ uma seção local para o campo $X_{H}$ em p. Assim, $\Sigma$ é uma subvariedade de codimensão $1 \mathrm{em} M$, satisfazendo:

$$
T_{q} \Sigma \oplus\left\{X_{H}(p)\right\}=T_{q} M, \forall q \in \Sigma .
$$

Consideremos $\psi: \Sigma \longrightarrow \Sigma$, a aplicação de Poincaré associada. Assim, ela é escrita na forma:

$$
\psi(q)=\varphi(T(q), q)
$$

onde $T: \Sigma \rightarrow \mathbb{R}$ é uma função diferenciável, que corresponde ao "tempo de retorno". Analisemos a diferencial de $\psi$ em $p$.

É possível mostrar a seguinte relação entre os polinômios característicos: 


$$
\operatorname{det}\left(\lambda I d-d_{p} \varphi_{T}\right)=(1-\lambda) \operatorname{det}\left(\lambda I d-d_{p} \psi\right)
$$

Concluímos ${ }^{2}$ dessa relação que:

$$
\sigma\left(d_{p} \varphi T\right)=\{1\} \bigcup \sigma\left(d_{p} \psi\right)
$$

Vamos analisar, agora, essa situação no correspondente nível de energia. Suponhamos $H(p)=E$ e $W_{E}=H^{-1}(E)$ um nível regular de energia. Definimos:

$$
\Sigma_{E}=\Sigma \cap W_{E}
$$

Pela conservação de energia, $\Sigma_{E}$ é um subconjunto de $\Sigma$ invariante por $\psi$. Definimos então a sua restrição:

$$
\psi_{0}: \Sigma_{E} \longrightarrow \Sigma_{E}, \psi_{0}=\left.\psi\right|_{\Sigma_{E}}
$$

Observemos, ainda, que $\Sigma_{E}$ é uma subvariedade de codimensão 2 em $M$, que está contida em $W_{E}$, nível regular de energia, e $\Sigma_{E}$ é transversal ao campo Hamiltoniano $X_{H}$. Segue, pelo exemplo 2.2.6, que $\Sigma_{E}$ é uma subvariedade simplética de $\left(M^{2 n}, \omega\right)$.

Indicando por $i: \Sigma_{E} \hookrightarrow M$ a inclusão em $M$ e definindo $\omega_{E}=i^{*} \omega$, 2-forma em $\Sigma_{E}$, temos:

Proposição 2.3.2 Nas condições acima, a aplicação $\psi_{0}: \Sigma_{E} \longrightarrow \Sigma_{E}$ é difeomorfismo tal que: $\psi_{0}^{*} \omega_{E}=\omega_{E}$. Além disso, vale:

$$
\sigma\left(d_{p} \varphi_{T}\right)=\{1,1\} \cup \sigma\left(d_{p} \psi_{0}\right)
$$

Demonstração: $\operatorname{Ver}$ [35].

Ou seja, a restrição da aplicação de Poincaré ao nível de energia da órbita é um simplectomorfismo de $\left(\Sigma_{E}, \omega_{E}\right)$.

Esses resultados sobre autovalores para Aplicações de Poincaré, em $\mathbb{R}^{4}$, serão resumidos no próximo Lema, da maneira que será aplicada neste texto.

Lema 2.3.1 Suponhamos $H: \mathbb{R}^{4} \longrightarrow \mathbb{R}$ uma função diferenciável e $p \in \mathbb{R}^{4}$ ponto cuja órbita, por $X_{H}$, é periódica com período $T>0$. Então valem as seguintes afirmações:

- $\sigma\left(d_{p} \phi_{T}^{H}: T_{p} \mathbb{R}^{4} \longrightarrow T_{p} \mathbb{R}^{4}\right)=\left\{1,1, \lambda, \lambda^{-1}\right\}$.

\footnotetext{
${ }^{2} \sigma$ denota o espectro da aplicação
} 
- Sejam $\Sigma$ uma seção transversal para a órbita periódica de p, em seu nível de energia, e $\psi: \Sigma \longrightarrow \Sigma$ a correspondente Transformação de Poincaré. Então:

$$
\sigma\left(d_{p} \psi: T_{p} \Sigma \longrightarrow T_{p} \Sigma\right)=\left\{\lambda, \lambda^{-1}\right\}
$$

Demonstração: Destacaremos os argumentos importantes na demonstração desse lema. Uma demonstração completa das afirmações, contidas neste lema, podem ser encontradas em [35].

- A forma dos autovalores de um simplectomorfismo linear.

- 1 é autovalor de $d_{p} \phi_{T}^{H}: T_{p} \mathbb{R}^{4} \longrightarrow T_{p} \mathbb{R}^{4}$, associado ao autovetor $X_{H}(p)$.

- Se $\psi: \Sigma \longrightarrow \Sigma$ é a Transformação de Poincaré, sobre o nível de energia, então $\Sigma$ é uma subvariedade simplética de $\left(\mathbb{R}^{4}, \omega_{0}\right)$ e $\psi$ é um simplectomorfismo. Assim, $\sigma\left(d_{p} \psi: T_{p} \Sigma \longrightarrow T_{p} \Sigma\right)=\left\{\lambda, \lambda^{-1}\right\}$.

- Vale a relação:

$$
\sigma\left(d_{p} \phi_{T}^{H}\right)=\{1,1\} \cup\left\{d_{p} \psi\right\}
$$

O próximo exemplo nos mostra como o Lema 2.3.1 pode ser útil para o estudo da dinâmica próxima a uma órbita periódica, restrita ao seu nível de energia, no momento de caracterizar o tipo de órbita em questão.

Exemplo 2.3.1 No contexto do Lema anterior, suponhamos suponhamos $\Sigma$ seção para a órbita de p e $\Sigma_{E}$ a restrição da seção ao nível de energia. Assim, $\operatorname{dim} \Sigma=3$ e dim $\Sigma_{E}=2$. Temos que $\Sigma_{E}$ é uma subvariedade simplética de $M$. Próximo a $p \in \Sigma_{E}$, podemos escolher o sistema de coordenadas do Teorema de Darboux. Assim, nessas coordenadas, $\omega_{E}=d x \wedge d y$ e a aplicação de Poincaré, sobre o nível de energia, pode ser vista como um difeomorfismo, definido numa vizinhança da origem, que preserva área.

Utilizando o Lema anterior, teremos os autovalores de $d_{p} \varphi_{T}$ como sendo da forma $\left\{1,1, \lambda, \frac{1}{\lambda}\right\}$, onde $\left\{\lambda, \frac{1}{\lambda}\right\}$ serão os autovalores da restrição da aplicação de Poincaré, $d_{p} \psi_{0}$, ao nivel de energia.

Se $\lambda \in \mathbb{R}$, com $|\lambda| \neq 1$, então a órbita periódica é hiperbólica no nível de energia e, pelo Teorema de Hartman-Grobman, a derivada $d_{p} \psi_{0}$ determina $o$ comportamento dinâmico local. Se $\lambda \in \mathbb{S}^{1} \backslash\{-1,1\}$, então a órbita é elíptica. Nesse caso, a primeira derivada é insuficiente para descrever a dinâmica local e precisamos recorrer a derivadas de ordem superior. Posteriormente, voltaremos a explorar essa situação. 


\subsection{Aplicações Twist Simpléticas}

Existe um modelo discreto de sistemas dinâmicos para o estudo de certos sistemas hamiltonianos, trata-se das aplicações twist simpléticas. Analisaremos algumas propriedades dessas aplicações, quando definidas em $\mathbb{S}^{1} \times \mathbb{R}$ (tais resultados se estendem a aplicações definidas em $\mathbb{S}^{1} \times[a, b]$, ver [14] para maiores detalhes). As aplicações twist podem ser definidas em $\mathbb{T}^{n} \times \mathbb{R}^{n}$, que corresponde ao fibrado contangente do toro n-dimensional, ou até mesmo em fibrados cotangentes mais gerais (ver [14]). Podemos pensar que estas aplicações representam a aplicação fluxo, de certos sistemas Hamiltonianos, em um instante $t_{0}$. A recíproca é verdadeira. Dada uma aplicação twist simplética, é possível encontrar um sistema Hamiltoniano, em geral dependente do tempo, que a represente, no sentido de ela ser o fluxo hamiltoniano num certo instante de tempo.

No que segue, adotaremos as seguintes notações:

- $\mathbb{S}^{1}=\mathbb{R} \backslash \mathbb{Z}$ ou $\mathbb{S}^{1}=\mathbb{R} \backslash 2 \pi \mathbb{Z}$ e $\mathbb{A}=\mathbb{S}^{1} \times \mathbb{R}$.

- Um ponto $x \in \mathbb{A}$ é representado pelas seguintes coordenadas $x=$ $(\theta, r) \in \mathbb{S}^{1} \times \mathbb{R}$.

- Em $\mathbb{A}$, temos a estrutura simplética usual, vinda do fibrado cotangente de $\mathbb{S}^{1}$ :

$$
\lambda=r d \theta \text { e } \omega=-d \lambda=d \theta \wedge d r .
$$

- $p: \mathbb{R}^{2} \longrightarrow \mathbb{A}$ é o recobrimento universal de $\mathbb{A}$.

- $\pi: \mathbb{R}^{2} \longrightarrow \mathbb{R}$ e $\pi: \mathbb{A} \longrightarrow \mathbb{S}^{1}$ representam as projeções na primeira coordenada.

- $V(x)=\operatorname{Ker}\left(d_{x} \pi\right), \pi: \mathbb{A} \longrightarrow \mathbb{S}^{1}$. Trata-se do subespaço vertical de $T_{x} \mathbb{A}$. Podemos identificar: $V(x)=\{\theta\} \times \mathbb{R}, x=(\theta, r)$.

A seguir, a definição de aplicação twist simplética, em $\mathbb{S}^{1} \times \mathbb{R}$

Definição 2.4.1 Seja $f: \mathbb{S}^{1} \times \mathbb{R} \longrightarrow \mathbb{S}^{1} \times \mathbb{R}$ um difeomorfismo de classe $C^{1}$, satisfazendo as seguintes condiçôes:

1. f é isotópica a Id de $\mathbb{A}$.

2. (Condição Twist) Existe $\epsilon>0$ tal que:

$$
\forall x \in \mathbb{A}, \epsilon<d_{x}(\pi \circ f)(0,1)<\frac{1}{\epsilon}
$$


3. $f$ é um simplectomorfismo exato de $\mathbb{A}$.

Nesse caso, dizemos que $f$ é uma aplicação twist simplética sobre $\mathbb{A}$.

Em relação a esta definição, faremos algumas observações.

\section{Observação 2.4.1 Temos:}

1. A primeira condição significa que $f$ preserva orientação e os bordos de $\mathbb{A}$. Trata-se de uma condição muito natural para aplicações que provém de fluxos.

2. A terceira condição nos diz que existe uma função diferenciável $s$ : $\mathbb{A} \longrightarrow \mathbb{R}$ tal que:

$$
d s=f^{*} \lambda-\lambda
$$

3. É possivel encontrar $F: \mathbb{R}^{2} \longrightarrow \mathbb{R}^{2}$ difeomorfismo de classe $C^{1}$, que seja levantamento de $f, F(x, y)=(X, Y)$, satisfazendo as seguintes condições:

- F é isotópica a Id.

- Existe $\epsilon>0$ tal que:

$$
\epsilon<\frac{\partial X}{\partial x}(x, y)<\frac{1}{\epsilon}, \forall(x, y) \in \mathbb{R}^{2} .
$$

- Existe uma função $S: \mathbb{R}^{2} \longrightarrow \mathbb{R}$, diferenciável, tal que:

$$
F^{*} y d x-y d x=d S
$$

Assim, F é um simplectomorfismo exato de $\mathbb{R}^{2}$.

4. A condição twist pode ser vista, então, como uma mudança de coordenadas:

$$
\begin{aligned}
\psi_{F} & : \mathbb{R}^{2} \longrightarrow \mathbb{R}^{2} \\
(x, y) & \mapsto(x, X(x, y))
\end{aligned}
$$

Nessas coordenadas, a seguinte relação é estabelecida entre F e S:

$$
F(x, y)=(X, Y) \Leftrightarrow \frac{\partial S}{\partial X}=Y e \frac{\partial S}{\partial x}=-y
$$

Essa equivalência nos permite caracterizar as órbitas de F como pontos críticos de um certo funcional. Exploraremos essa relação mais a frente. 
Vejamos a alguns exemplos.

Exemplo 2.4.1 Seja $f: \mathbb{A} \longrightarrow \mathbb{A}$, dada por:

$$
f(\theta, r)=\left(\theta+\alpha+\alpha_{1} r, r\right)
$$

Onde $\alpha_{1} \neq 0$. Temos que $f$ é uma aplicação twist simplética. Observemos que $\mathbb{A}$ é folheado por círculos invariantes por $f$ :

$$
\mathbb{A}=\bigcup_{r \in \mathbb{R}} \mathbb{S}^{1} \times\{r\}
$$

Além disso, a dinâmica de $f$, sobre cada um desses círculos invariantes, é topologicamente conjugada a uma rotação em $\mathbb{S}^{1}$.

Exemplo 2.4.2 Seja $\epsilon>0$. Definimos:

$$
\begin{gathered}
f_{\epsilon}: \mathbb{A} \longrightarrow \mathbb{A} \\
(\theta, r) \mapsto\left(\theta+r+\frac{\epsilon}{2 \pi} \sin 2 \pi \theta, r+\frac{\epsilon}{2 \pi} \sin 2 \pi \theta\right)
\end{gathered}
$$

Para $\epsilon=0$, temos o exemplo anterior. A medida que $\epsilon$ cresce, aqueles círculos invariantes do exemplo anterior vão deixando de existir. Para $\epsilon>0$, suficientemente grande, nenhum círculo irá "sobreviver".

A seguir, enunciaremos um resultado que nos permitirá dar outros exemplos. Além disso, esse resultado estabelece uma relação entre aplicações twist simpléticas e sistemas Hamiltonianos no $\mathbb{R}^{2}$, conforme mencionado no início desta subseção.

Proposição 2.4.1 Suponhamos:

$$
\begin{gathered}
H: \mathbb{R}^{2} \times \mathbb{R} \longrightarrow \mathbb{R} \\
((x, y), t) \mapsto H_{t}(x, y)
\end{gathered}
$$

Função de classe $C^{2}$, com as seguintes propriedades:

1. $H_{t}$ é $2 \pi$-periódica em $x$ e periódica em $t$.

2. $\forall(x, y) \in \mathbb{R}^{2}, \frac{\partial^{2} H_{t}}{\partial y^{2}}(x, y)>0$.

3. Existe $K>0$, tal que:

$$
\sup _{\mathbb{R}^{2}}\left\|d X_{H_{t}}\right\|<K
$$


então, existe $\delta>0$ tal que se $t \in(0, \delta)$, então:

$$
\phi_{t}^{H}: \mathbb{R}^{2} \longrightarrow \mathbb{R}^{2}
$$

é o levantamento de uma aplicação twist simplética em $\mathbb{A}$.

Demonstração: Ver em [14].

Por meio dessa proposição, temos os seguintes exemplos:

Exemplo 2.4.3 Consideremos a Hamiltoniana do pêndulo, analisada no exemplo 2.1.2. Aplicando a proposição anterior, é possivel verificar que existe $\delta>0$, tal que para $t \in(0, \delta)$, o fluxo hamiltoniano, associado, no instante $t, \phi_{t}^{H}: \mathbb{R}^{2} \rightarrow \mathbb{R}^{2}$, será o levantamento de uma aplicação twist simplética $f: \mathbb{A} \rightarrow \mathbb{A}$, onde $\mathbb{A}=\mathbb{S}^{1} \times \mathbb{R}$. Projetando-se a análise feita, no exemplo 2.1.2, em $\mathbb{A}$, obtemos a situação esboçada na Figura 2.2

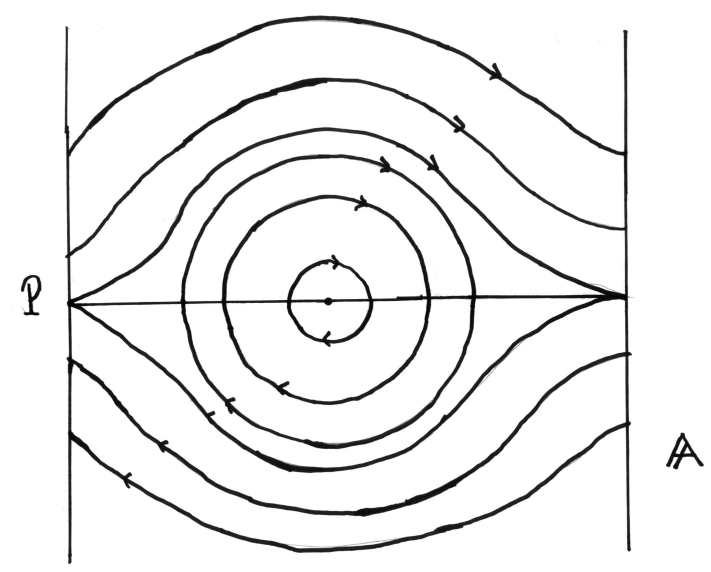

Figura 2.2: Curvas invariantes para a aplicação do exemplo 2.4.3.

Este exemplo mostra uma aplicação twist que possui muitas curvas invariantes. Devemos notar que o ponto $P$, que é a projeção dos pontos $P_{n}$ (ver exemplo 2.1.2), é um ponto fixo hiperbólico de $f$. Nesse caso, as variedades invariantes serão a união de duas curvas invariantes por $f$, que são gráficos Lipschtiz sobre $\mathbb{S}^{1}$. Estes gráficos são Lipschtiz, mas não são suaves.

Exemplo 2.4.4 Consideremos, agora, a Hamiltoniana do exemplo 2.1.3. Aplicando a proposição 2.4.1, existe $\delta>0$, tal que para $t \in(0, \delta)$, o fluxo hamiltoniano, associado, no instante $t, \phi_{t}^{H_{\epsilon}}: \mathbb{R}^{2} \rightarrow \mathbb{R}^{2}$, será o levantamento de uma aplicação twist simplética $f_{\epsilon}: \mathbb{A} \rightarrow \mathbb{A}$. Observemos que $f_{0}$ é aplicação 
analisada no exemplo anterior. Projetando-se em $\mathbb{A}$ a análise feita no exemplo 2.1.3, para cada $\epsilon \neq 0$, pequeno, teremos um ponto fixo hiperbólico $P_{\epsilon}$ de $f_{\epsilon}$. Nesse caso, as variedades invariantes associadas deixam de coincidir, como no caso $\epsilon=0$ e passam a se interceptar transversalmente, produzindo a existência de pontos homoclínicos transversais. Este sistema possui entropia topológica positiva.

Observação 2.4.2 Analisando os dois últimos exemplos, podemos dizer que a perturbação destruiu as curvas invariantes do exemplo 2.4.3, que correspondem às separatrizes do ponto fixo hiperbólico. A destruição destas curvas invariantes pela aplicação twist $f$, produziu um ponto homoclinico transversal. Essa situação em que temos uma aplicação twist $f_{\epsilon}=f_{0}+o(\epsilon)$, em que a perturbação destrói uma curva invariante para a aplicação $f_{0}$, produzindo um ponto homoclínico transversal, e, portanto, entropia topológica positiva, será explorada neste texto.

Como pode ser observado, por meio desses exemplos, "esses círculos invariantes" desempenham um papel importante no estudo da aplicações twist. Faremos, desse conceito, um guia para o estudo dessas aplicações.

Consideremos $f: \mathbb{A} \longrightarrow \mathbb{A}$ uma aplicação twist simplética e $F: \mathbb{R}^{2} \longrightarrow$ $\mathbb{R}^{2}$ levantamento de $f$. Para analisar as propriedades desses "círculos invariantes", temos a seguinte definição.

Definição 2.4.2 Uma curva rotacional invariante para a aplicação twist $f$ é um subconjunto $\Gamma \subseteq \mathbb{A}$, satisfazendo as seguintes propriedades:

1. Г é uma curva $C^{0}$, fechada e sem auto-interseções.

2. $\Gamma$ é invariante por $f$. Ou seja $f(\Gamma)=\Gamma$.

3. $\Gamma$ é homotopicamente não trivial.

Sobre a estrutura desses conjuntos, temos o seguinte resultado.

Proposição 2.4.2 Seja $\Gamma \subseteq \mathbb{A}$ uma curva rotacional invariante para $f$. Então, existe uma função $\psi: \mathbb{S}^{1} \longrightarrow \mathbb{R}$, função Lipschitz, tal que:

$$
\Gamma=\operatorname{Graf}(\psi)=\left\{(x, \psi(x)): x \in \mathbb{S}^{1}\right\}
$$

Demonstração: Ver [23].

Portanto, se $\Gamma \subseteq \mathbb{A}$ é uma curva rotacional invariante para $f$, então $\Gamma$ é um gráfico Lipschitz sobre sua projeção $\mathbb{S}^{1}$. Seguirá, então, que a projeção $\left.\pi\right|_{\Gamma}: \Gamma \longrightarrow \mathbb{S}^{1}$ é um homeomorfismo. Logo, como $\Gamma$ é invariante por $f$, a aplicação $\left.\pi\right|_{\Gamma}$ projeta a dinâmica de $f$ sobre $\mathbb{S}^{1}$. Sendo assim, torna-se natural tentar definir a noção de número de rotação de uma órbita. 
Definição 2.4.3 Seja $\left(x_{k}, y_{k}\right)_{k \in \mathbb{Z}}$ uma órbita de $F$. O número de rotação da órbita é definido pelo seguinte limite, quando existe:

$$
\lim _{k \longrightarrow \pm \infty} \frac{x_{k}-x_{0}}{k}
$$

Seja $g: \mathbb{S}^{1} \longrightarrow \mathbb{S}^{1}$, definido por:

$$
g=\left(\left.\pi\right|_{\Gamma}\right) \circ f \circ\left(\left.\pi\right|_{\Gamma}\right)^{-1}
$$

Temos que $g$ é um homeomorfismo de $\mathbb{S}^{1}$ que preserva orientação. Segue, então, que fica associado um número de rotação ao homeomorfismo $g$, ou melhor, fica associado um número de rotação às órbitas de $f$ em $\Gamma$. Utilizando a teoria de Poincaré para homeomorfismos de $\mathbb{S}^{1}$, temos uma boa descrição das possibilidades para a dinâmica de $f$ sobre uma curva rotacional invariante $\Gamma \subseteq \mathbb{A}$. Maiores detalhes podem ser encontrados em [16].

É importante destacar que o conjunto de todas as curvas rotacionais invariantes para a aplicação $f$, que denotaremos por $C(f)$, pode ser vazio, como foi observado no exemplo 2.4.2. Além disso, o conjunto $C(f)$ é um subconjunto fechado de $\mathbb{A}$, pois cada curva é um gráfico invariante Lipschitz, com constante Lipschitz limitada, dependendo da aplicação $f$ (ver [2]). Analisaremos algumas propriedades do complementar de $C(f)$ em $\mathbb{A}, \mathbb{U}(f)=\mathbb{A} \backslash C(f)$.

Uma curva $C \subseteq \mathbb{A}$, fechada, contínua e mergulhada em $\mathbb{A}$, é chamada curva rotacional quando é homotopicamente não trivial. Um subconjunto $A \subseteq \mathbb{A}$ é chamado sub anel essencial quando é homeomorfo a $\mathbb{A}$ e contém uma curva rotacional.

Em relação às componentes conexas de $\mathbb{U}(f)$, temos a seguinte proposição:

Proposição 2.4.3 Toda componente conexa de $\mathbb{U}(f)$ ou é um disco topológico limitado ou é um sub anel essencial. Além disso, temos:

1. Quando uma tal componente é um disco topológico $\mathbb{D}$, esse disco é periódico, isto é, existe $N \in \mathbb{N}$ tal que $f^{N}(\mathbb{D})=\mathbb{D}$.

2. No caso de uma componente conexa ser um sub anel essencial, o bordo dela será ou um gráfico invariante, ou $\mathbb{S}^{1} \times\{+\infty\}$ ou $\mathbb{S}^{1} \times\{-\infty\}$.

Demonstração: Ver [2].

No caso do exemplo 2.4.3, dado anteriormente, é simples observar, projetando o retrato de fase do pêndulo em $\mathbb{A}$, uma componente conexa do tipo disco. Componentes do tipo sub anel essencial podem ser observadas no exemplo 2.4.2. Muitos fenômenos dinâmicos interessantes de uma aplicação twist simplética ocorrem nas componentes conexas tipo sub anel essencial (componente anelar). 
Definição 2.4.4 Uma componente anelar de $\mathbb{U}(f)$ é chamada zona de instabilidade de $f$.

Em relação a essas regiões, temos:

Proposição 2.4.4 Seja $A \subseteq \mathbb{A}$, uma zona de instabilidade para $f$, tal que $\partial A=C_{-} \cup C_{+} . E_{n} t \tilde{a} O^{3}$ :

$$
W^{u}\left(C_{-}\right) \cap W^{s}\left(C_{+}\right) \bigcap A \neq \emptyset
$$

Demonstração: Ver em [6] e [20].

Um belo resultado sobre a dinâmica de uma aplicação twist, que relaciona curvas rotacionais invariantes com entropia topológica e será fundamental para concluirmos o Corolário 1.1.1, é apresentado a seguir.

Proposição 2.4.5 Suponhamos $f: \mathbb{S}^{1} \times[a, b] \rightarrow \mathbb{S}^{1} \times[a, b]$ uma aplicação twist, definida sobre o anel compacto, tal que a restrição de $f$ aos bordos tenha números de rotação $\rho_{0}$ e $\rho_{1}$, com $\rho_{0}<\rho_{1}$. Se $e^{4} h_{\text {top }}(f)=0$, então $f$ possui uma curva rotacional invariante, com número de rotação $\omega$, com $\omega \in\left(\rho_{0}, \rho_{1}\right)$.

Demonstração Ver em [1]. Há, também, uma discussão sobre este resultado em [14].

Observação 2.4.3 Em [1], estuda-se a dinâmica de uma aplicação twist $f$, em anéis, que formam zonas de instabilidade. Em particular, é possível construir um conjunto compacto invariante $K$, associado a certas órbitas (construídas a partir de sub e super soluções do princípio variacional), tal que $\left.f^{q}\right|_{K}$, para algum $q \in \mathbb{N}$, é semi-conjugada a um shift de Bernoulli.

\subsection{Teoria de Aubry Mather}

Como foi observado no exemplo 2.4.2, ao variarmos o parâmetro $\epsilon$, curvas rotacionais invariantes vão sendo destruídas. Para $\epsilon$ suficientemente grande, tais curvas deixam de existir. Poderíamos nos questionar se alguma propriedade relacionada a essas curvas ainda persiste no sistema, mesmo após sua destruição. Essa questão pode ser analisada através de um princípio variacional associado a aplicações twist. A perturbação do sistema pode destruir a curva rotacional como objeto geométrico do sistema, mas as propriedades

\footnotetext{
${ }^{3} W^{u}$ e $W^{s}$ denotam as variedades instável e estável associada ao respectivo conjunto.

${ }^{4} h_{t o p}(f)$ representa a entropia topológica da aplicação $f$.
} 
variacionais da mesma persistem. Dentro desse ponto de vista, poderíamos dizer que a curva não seria destruída, mas seria transformada em um outro tipo de conjunto, que também é um gráfico, porém pode-se mudar as propriedades topológicas e de regularidade das curvas rotacionais. Esses novos conjuntos são os chamados conjuntos de Aubry Mather. Faremos uma breve estudo sobre eles.

Iremos supor ao longo dessa seção que $f: \mathbb{A} \longrightarrow \mathbb{A}$ é uma aplicação twist simplética, com levantamento fixado $F: \mathbb{R}^{2} \longrightarrow \mathbb{R}^{2}$ e função geradora $S: \mathbb{R}^{2} \longrightarrow \mathbb{R}$.

Conforme foi mencionado, é possível associar um princípio variacional às órbitas de uma aplicação twist. Fixemos $m, n \in \mathbb{Z}$, com $m>n$, e $a, b \in \mathbb{R}$. Consideremos o seguinte conjunto:

$$
\Omega_{a, b}^{n, m}=\left\{\left(x_{n}, \ldots, x_{m}\right) \in \mathbb{R}^{m-n+1}: x_{n}=a \text { e } x_{m}=b\right\}
$$

Notemos que $\Omega_{a, b}^{n, m}$ pode ser identificado como conjunto de sequências finitas que conecta o ponto $a \in \mathbb{R}$, no instante $n$, ao ponto $b \in \mathbb{R}$, no instante $m$, como "um caminho discreto". A comparação com um espaço de curvas absolutamente contínuas para se definir a ação de um sistema Lagrangiano é muito instrutiva, como pode ser observado em [19] e [3]. Observemos ainda que podemos fazer a seguinte identificação $\Omega_{a, b}^{n, m} \approx \mathbb{R}^{m-n-1}$.

Definimos o seguinte funcional:

$$
W: \Omega_{a, b}^{n, m} \longrightarrow \mathbb{R}
$$

Dado por:

$$
W(x)=\sum_{i=n}^{m-1} S\left(x_{i}, x_{i+1}\right)
$$

A associação entre órbitas de $F$ e pontos críticos desse funcional é dada pela seguinte proposição:

Proposição 2.5.1 Um segmento $\left(x_{n}, \ldots, x_{m}\right) \in \Omega_{a, b}^{n, m}$ é a projeção de uma órbita $\left(x_{k}, y_{k}\right)_{k \in \mathbb{Z}}$ de $F$, satisfazendo $x_{n}=a$ e $x_{m}=b$ se, e somente se, é ponto crítico de $W$.

Isso nos permite falar em órbitas minimizantes, como aquelas órbitas em que qualquer segmento finito é minimizante para o respectivo funcional ação. Ou seja, é um ponto de mínimo do correspondente funcional $W$. Nesse sentido, temos a seguinte propriedade de curvas rotacionais invariantes.

Proposição 2.5.2 Seja $\Gamma \subseteq \mathbb{A}$ uma curva rotacional invariante para a aplicação $f$. Então, o levantamento de toda órbita de $\Gamma$ é minimizante. 
Essa propriedade de minimização das curvas rotacionais invariantes é uma propriedade interessante no sentido de não estar relacionada a métodos perturbativos.

É possível modificar esse princípio de pontos críticos, na proposição 2.5.1, de modo a se analisar apenas órbitas periódicas.

Se $\left(x_{k}\right)_{k \in \mathbb{Z}}$ é uma sequência de reais, então dizemos que $\left(x_{k}\right)_{k \in \mathbb{Z}}$ é tipo $(m, n)$, quando:

$$
x_{k+n}=x_{k}+m, \forall k \in \mathbb{Z} .
$$

Observemos que se $\left(x_{k}, y_{k}\right)_{k \in \mathbb{Z}}$ é uma órbita de $F$, que é o levantamento de uma órbita periódica de $f$, então existem $(m, n) \in \mathbb{Z} \times \mathbb{N}$, tal que:

$$
x_{k+n}=x_{k}+m \text { e } y_{k+n}=y_{k}, \forall k \in \mathbb{Z} .
$$

Nesse sentido, diremos que a órbita é tipo $(m, n)$. É possível mostrar, ainda, que órbitas periódicas tipo $(m, n)$ possuem número de rotação, segundo a definição 2.4.3, dado por $\frac{m}{n}$.

Indiquemos por $\Omega_{m, n}$ o espaço das sequências reais do tipo $(m, n)$. Observemos que um elemento $x \in \Omega_{m, n}$ fica completamente determinado por $n$ pontos, $\left(x_{1}, \ldots, x_{n}\right)$. Portanto, identificamos $\Omega_{m, n} \approx \mathbb{R}^{n}$. Definimos o seguinte funcional:

$$
W_{m, n}: \Omega_{m, n} \longrightarrow \mathbb{R}
$$

Dado por:

$$
W_{m, n}(x)=S\left(x_{n}, x_{1}+m\right)+\sum_{i=1}^{n-1} S\left(x_{i}, x_{i+1}\right) .
$$

Temos o seguinte princípio de pontos críticos:

Proposição 2.5.3 Um ponto $x \in \Omega_{m, n}$ é a projeção de uma órbita tipo $(m, n)$ para $F$ se, e somente se, é ponto crítico de $W_{m, n}$.

Observemos que se $x_{k}$ é ponto crítico de $W_{m, n}$, então, naturalmente, temos uma órbita periódica. De fato, teremos:

$y_{k+n}=-\frac{\partial S}{\partial x}\left(x_{k+n}, x_{k+1+n}\right)=-\frac{\partial S}{\partial x}\left(x_{k}+m, x_{k+1}+m\right)=-\frac{\partial S}{\partial x}\left(x_{k}, x_{k+1}\right)=y_{k}$.

Isso vale para todo $k \in \mathbb{Z}$.

Gostaríamos de analisar, agora, a existência de órbitas periódicas do tipo $(m, n)$, que sejam minimizantes. Para isso, faremos uso do seguinte lema auxiliar, que é uma consequência da condição twist. 
Lema 2.5.1 Vale o seguinte limite:

$$
\lim _{\|X-x\| \longrightarrow \infty} S(x, X)=+\infty .
$$

Consequência disso será o fato de que se $\left(y_{k}\right)_{k} \in\left\{y \in \Omega_{m, n}, y_{1} \in[0,1]\right\}$ e $\lim _{k \rightarrow \infty}\left\|y_{k}\right\|=+\infty$, então $\lim _{k \longrightarrow \infty} W_{m, n}\left(y_{k}\right)=+\infty$. Segue então que se $k>0$ é suficientemente grande, então $W_{m, n}^{-1}(-\infty, k] \bigcap\left\{y \in \Omega_{m, n}: y_{1} \in[0,1]\right\}$ é limitado e, por continuidade, é fechado. Logo é compacto e $W_{m, n}$ alcançará um mínimo neste conjunto. Com isso, esboçamos a ideia da prova de parte da seguinte proposição:

Proposição 2.5.4 Dados $(m, n) \in \mathbb{Z} \times \mathbb{N}, W_{m, n}: \Omega_{m, n} \longrightarrow \mathbb{R}$ possui um ponto de mínimo. Assim, existem órbitas minimizantes com todo número de rotação racional. Além disso, por um processo de limite, é possível concluir a existência de órbitas minimizantes com número de rotação irracional.

Assim, existem órbitas minimizantes com todo número de rotação real. Gostaríamos de analisar, num certo sentido, o conjunto formado por todas as órbitas, com um dado número de rotação, e que sejam minimizantes. Para melhor caracterizá-lo, vamos introduzir as seguintes definições.

Definição 2.5.1 Um subconjunto $M \subseteq \mathbb{R}^{2}$ é dito F-ordenado quando for invariante por $F$ e satisfizer a seguinte condição:

Se $z_{1}, z_{2} \in M, \operatorname{com} \pi_{1}\left(z_{1}\right)<\pi_{1}\left(z_{2}\right)$, então: $\pi_{1}\left(F\left(z_{1}\right)\right)<\pi_{1}\left(F\left(z_{2}\right)\right)$,

onde $\pi_{1}: \mathbb{R}^{2} \longrightarrow \mathbb{R}$ é a projeção na primeira coordenada. A seguir, a definição de conjunto de Aubry Mather.

Definição 2.5.2 Um conjunto de Aubry Mather para $F$ é um subconjunto $M \subseteq \mathbb{R}^{2}$ que é fechado, F-ordenado e invariante por translações inteiras.

Quando falarmos em conjuntos de Aubry Mather, estaremos nos referindo a eles como subconjuntos do $\mathbb{R}^{2}$ ou suas projeções em $\mathbb{S}^{1} \times \mathbb{R}$. A seguir, descreveremos algumas propriedades desses conjuntos.

Proposição 2.5.5 Seja $M \subseteq \mathbb{R}^{2}$ conjunto de Aubry Mather para F. Então:

1. Todas as órbitas de $M$ possuem o mesmo número de rotação.

2. $M$ é um gráfico Lipschtiz sobre sua projeção $\pi(M)$. 
3. A dinâmica de $f$ sobre $p(M)$ é topologicamente conjugada à dinâmica de um homeomorfismo $\mathrm{g}: \mathbb{S}^{1} \longrightarrow \mathbb{S}^{1}$, que preserva orientação, sobre um subconjunto fechado e invariante.

É possível construir diversos tipos de conjuntos de Aubry Mather para uma aplicação twist. Podemos dar o seguinte exemplo de conjuntos de Aubry Mather. Suponhamos $\alpha \in \mathbb{R}$. Consideremos o conjunto $M_{\alpha} \subseteq \mathbb{R}^{2}$ formado pelo fecho de todas as translações inteiras de órbitas minimizantes, com número de rotação $\alpha$. Assim, $M_{\alpha}$ é um conjunto de Aubry Mather. Observemos que uma curva rotacional invariante é um conjunto de Aubry Mather, formado a partir de órbitas minimizantes. A seguir, resumiremos os resultados desta seção, relacionando-os com os conjuntos de Aubry Mather.

Proposição 2.5.6 Seja $f: \mathbb{A} \longrightarrow \mathbb{A}$ aplicação twist simplética, com levantamento $F: \mathbb{R}^{2} \longrightarrow \mathbb{R}^{2}$. temos:

1. F possui órbitas minimizantes com todo número de rotação em $\mathbb{R}$.

2. Essas órbitas minimizantes estão contidas em certos conjuntos fechados e invariantes, os conjuntos de Aubry Mather, que são gráficos Lipschtiz sobre suas projeções.

3. A dinâmica de $f$, sobre esses conjuntos, é topologicamente conjugada à dinâmica de um homeomorfismo de $\mathbb{S}^{1}$, que preserva orientação, sobre um subconjunto fechado e invariante de $\mathbb{S}^{1}$.

Esse teorema nos permite analisar os tipos de dinâmicas de uma aplicação twist sobre um conjunto de Aubry Mather, como também as possíveis estruturas topológicas desses conjuntos. Suponhamos $M \subseteq \mathbb{R}^{2}$ conjunto de Aubry Mather, com número de rotação $\alpha \in \mathbb{R}$. Então $\alpha \in \mathbb{Q}$ ou $\alpha \notin \mathbb{Q}$. Assim, temos as possibilidades ${ }^{5}$ :

- $\alpha \in \mathbb{Q}$; Nesse caso, $p(M)$ ou é uma coleção de órbitas periódicas, com, possivelmente órbitas heteroclínicas, conectando duas delas, ou uma curva rotacional invariante com número de rotação racional.

- $\alpha \notin \mathbb{Q}$; Nesse caso, $p(M)$ ou é um conjunto de Cantor, com, possivelmente, órbitas homoclínicas em seus gaps ou é uma curva rotacional invariante com número de rotação irracional.

\footnotetext{
${ }^{5}$ Consequência de rsultados de dinâmica em $\mathbb{S}^{1}$. Ver [16].
} 
Sendo assim, ao destruirmos curvas rotacionais invariantes, como no exemplo 2.4.2, temos, ainda, um certo tipo de estrutura, a dos Conjuntos de Aubry Mather, que ainda mantém algumas relações com as curvas rotacionais invariantes. 


\section{Capítulo 3}

\section{Propriedades Dinâmicas de $X_{H_{\epsilon}}$}

O objetivo deste capítulo é provar o Teorema 1.1.1, que afirma que a órbita periódica $\Gamma_{E}$, de $X_{H_{\epsilon}}$, é elíptica em certos níveis de energia, quando $|\epsilon|$ é pequeno. Além disso, construiremos a seção transversal para a análise da correspondente Transformação de Poincaré, sobre o nível de energia e faremos algumas considerações sobre a dinâmica deste campo vetorial.

\subsection{Alguns resultados relacionados.}

Consideremos a seguinte Hamiltoniana, dependendo de uma parâmetro $\epsilon$, $H_{\epsilon}: \mathbb{R}^{4} \rightarrow \mathbb{R}$, dada pela igualdade (1.1). Em relação ao termo $R_{\epsilon}=$ $R_{\epsilon}\left(x_{1}, x_{2}\right)$, temos a seguinte observação.

Observação 3.1.1 Na maioria dos cálculos que faremos, é suficiente supor que $R_{\epsilon}\left(x_{1}, x_{2}\right)=\epsilon x_{1}^{2} x_{2}^{2}$. De fato, os outros termos se anulam nos cálculos que faremos.

Devido a esta observação, podemos supor que a função $H_{\epsilon}: \mathbb{R}^{4} \rightarrow \mathbb{R}$ tenha a seguinte forma:

$$
H_{\epsilon}\left(x_{1}, x_{2}, y_{1}, y_{2}\right)=\frac{y_{1}^{2}+y_{2}^{2}}{2}+\frac{\alpha^{2} x_{1}^{2}}{2}-\frac{\nu^{2} x_{2}^{2}}{2}+\frac{b_{1} x_{1}^{4}}{4}+\frac{b_{2} x_{2}^{4}}{4}-\epsilon x_{1}^{2} x_{2}^{2},
$$

onde $\alpha, \nu \neq 0$ e $b_{1}, b_{2}>0$. Temos o Campo Hamiltoniano:

$$
X_{H_{\epsilon}}=\left(y_{1}, y_{2},-\alpha^{2} x_{1}-b_{1} x_{1}^{3}+2 \epsilon x_{1} x_{2}^{2}, \nu^{2} x_{2}-b_{2} x_{2}^{3}+2 \epsilon x_{2} x_{1}^{2}\right) .
$$

E a equação de Hamilton: 


$$
\left\{\begin{array}{l}
x_{1}^{\prime}=y_{1} \\
x_{2}^{\prime}=y_{2} \\
y_{1}^{\prime}=-\alpha^{2} x_{1}-b_{1} x_{1}^{3}+2 \epsilon x_{1} x_{2}^{2} \\
y_{2}^{\prime}=\nu^{2} x_{2}-b_{2} x_{2}^{3}+2 \epsilon x_{2} x_{1}^{3}
\end{array}\right.
$$

Observemos que se $\epsilon=0$, então o comportamento desse sistema nas variáveis $\left(x_{1}, y_{1}\right)$ é independente do comportamento do sistema nas variáveis $\left(x_{2}, y_{2}\right)$. Dizemos, nessa situação, que o sistema está desacoplado. O comportamento do sistema nas variáveis $\left(x_{1}, y_{1}\right)$ é dado por um Hamiltoniano $H_{1}=H_{1}\left(x_{1}, y_{1}\right)$ e o comportamento do sistema nas variáveis $\left(x_{2}, y_{2}\right)$ é dado por um Hamiltoniano $H_{2}=H_{2}\left(x_{2}, y_{2}\right)$. $H_{1}$ e $H_{2}$ podem ser escritos como:

$$
\left\{\begin{array}{l}
H_{1}\left(x_{1}, y_{1}\right)=\frac{y_{1}^{2}}{2}+V_{1}\left(x_{1}\right)=\frac{y_{1}^{2}}{2}+\frac{\alpha^{2} x_{1}^{2}}{2}+\frac{b_{1} x_{1}^{4}}{4}, \\
H_{2}\left(x_{2}, y_{2}\right)=\frac{y_{2}^{2}}{2}+V_{2}\left(x_{2}\right) \frac{y_{2}^{2}}{2}-\frac{\nu^{2} x_{1}^{2}}{2}+\frac{b_{2} x_{1}^{4}}{4} .
\end{array}\right.
$$

Para $\epsilon \neq 0$, o sistema não está mais desacoplado, porém ainda vale:

Lema 3.1.1 Sejam $M_{i}=\left\{\left(x_{1}, x_{2}, y_{1}, y_{2}\right) \in \mathbb{R}^{4}:\left(x_{i}, y_{i}\right)=(0,0)\right\}$, onde $i=1$ ou $i=2$. Então valem:

- $M_{1}$ e $M_{2}$ são invariantes pelo fluxo do campo $X_{H_{\epsilon}}$;

- O fluxo de $X_{H_{\epsilon}}$ em $M_{1}$ é conjugado ao fluxo de $X_{H_{2}}$ e o fluxo de $X_{H_{\epsilon}}$ em $M_{2}$ é conjugado ao fluxo de $X_{H_{1}}$.

Demonstração: $O$ campo $X_{H_{\epsilon}}$ é tangente a $M_{1}$ e a $M_{2}$. Para o caso $M_{1}$, temos naturalmente um difeomorfismo $\pi_{2}: M_{1} \rightarrow \mathbb{R}^{2}$ e se $z \in M_{1}$, então $d_{z} \pi_{2}\left(X_{H_{\epsilon}}(z)\right)=X_{H_{2}}\left(\pi_{2}(z)\right)$. Segue destes fatos que $\pi_{2}$ é uma conjugação entre os fluxos dos dois campos. O caso $M_{2}$ é análogo.

Os retratos de fase dos campos bidimensionais $X_{H_{1}}$ e $X_{H_{2}}$ são esboçados nas Figura 3.1 e Figura 3.2, respectivamente.

Nesse ponto, podemos demonstrar o Lema 1.1.1, enunciado na introdução. Demonstração do Lema 1.1.1:Fixemos um nível de energia $E>0$ e seja $z_{0}=\left(0,0,0, y_{2}^{E}\right) \in \mathbb{R}^{4}$, onde $H_{2}\left(0, y_{2}^{E}\right)=E$. Pelo lema anterior, segue que a órbita de $z_{0}$, por $X_{H_{\epsilon}}$, é periódica, para todo $\epsilon \in \mathbb{R}$, e seu período não depende do parâmetro $\epsilon$. Além disso, esta órbita tem a seguinte forma:

$$
\phi_{t}^{H_{\epsilon}}\left(z_{0}\right)=\left(0, x_{2}(t), 0, y_{2}(t)\right)
$$




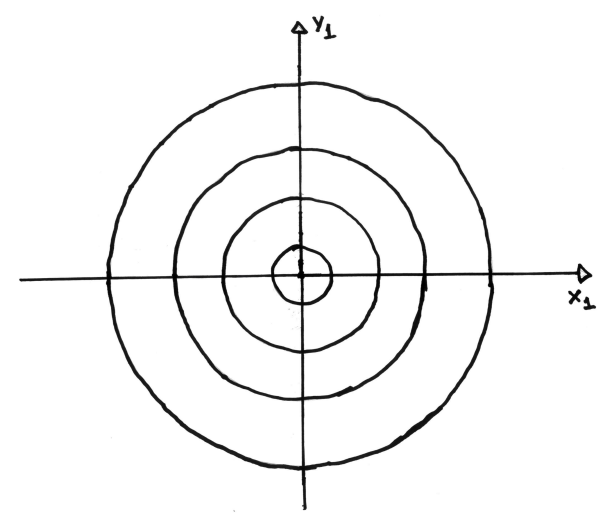

Figura 3.1: Retrato de Fase do sistema Hamiltoniano $X_{H_{1}}$

Onde $t \mapsto\left(x_{2}(t), y_{2}(t)\right)$ é uma órbita periódica de $X_{H_{2}}$, órbita com energia $E$. Nas condições em que fixamos o ponto $z_{0}$, o período de sua órbita pode ser visto como função da energia, no Sistema Hamiltoniano $H_{2}$. Isso conclui a demonstração do Lema 1.1.1.

Um dos objetivos, desta seção, é analisar como o tipo de órbita de $z_{0}$, em seu respectivo nível de energia (elíptica ou hiperbólica), depende do nível de energia $E$ e do parâmetro $\epsilon$. Nesse sentido, teremos:

Teorema 3.1.1 Suponhamos que o período $T$ da órbita de $z_{0}$ e o coeficiente $\alpha$ satisfaçam: $\alpha T \notin\{2 k \pi: k \in \mathbb{Z}\}$. Então existe $\epsilon_{0}>0$ tal que se $\epsilon \in$ $\left(-\epsilon_{0}, \epsilon_{0}\right)$, a órbita de $z_{0}$ é periódica elíptica em seu nível de energia.

A classificação do tipo de órbita periódica de $z_{0}$, em seu nível de energia, está relacionada aos autovalores da derivada $d_{z_{0}} \phi_{T}^{H_{\epsilon}}: T_{z_{0}} \mathbb{R}^{4} \longrightarrow T_{z_{0}} \mathbb{R}^{4}$. Por meio dessa relação, é possível reduzir a análise da classificação da órbita periódica de $z_{0}$, em seu nível de energia, a uma análise de uma equação diferencial unidimensional de segunda ordem. De fato, definindo $u_{\epsilon}: \mathbb{R} \longrightarrow \mathbb{R}$ por $^{1}$ :

$$
u_{\epsilon}(t)=-\alpha^{2}+2 \epsilon x_{2}^{2}(t)
$$

Consideremos a equação:

$$
x^{\prime \prime}=u_{\epsilon}(t) x
$$

Sejam $x_{1}=x_{1}(t)$ e $x_{3}=x_{3}(t)$ as soluções desta equação, caracterizadas pelas seguintes condições iniciais:

\footnotetext{
${ }^{1} x_{2}=x_{2}(t)$ é a correspondente coordenada (coordenada $x_{2}$ ) da órbita de $z_{0}$.
} 


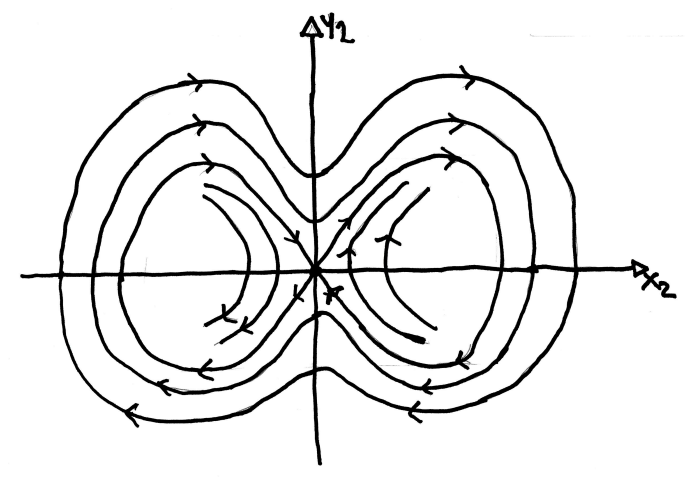

Figura 3.2: Retrato de Fase do sistema Hamiltoniano $X_{H_{2}}$. Nessa figura não está sendo detalhado o comportamento das trajetórias que não interceptam o eixo $y_{2}$, pois essas trajetórias não interferem na análise feita neste texto.

$$
\left\{\begin{array}{l}
x_{1}(0)=1, x_{1}^{\prime}(0)=0 ; \\
x_{3}(0)=0, x_{3}^{\prime}(0)=1 ;
\end{array}\right.
$$

A partir destas duas soluções específicas, definimos a seguinte função:

$$
\Phi_{\epsilon}(t)=\frac{x_{1}(t, \epsilon)+x_{3}^{\prime}(t, \epsilon)}{2}
$$

Por meio desta função $\Phi=\Phi_{\epsilon}(t)$, podemos classificar a órbita de $z_{0}$, em seu nível de energia:

Proposição 3.1.1 Se $\left(\Phi_{\epsilon}(t)\right)^{2} \in(0,1)$, então a órbita de $z_{0}$ é elíptica em seu nível de energia. Se $\left(\Phi_{\epsilon}(t)\right)^{2}>1$, então a órbita de $z_{0}$ é hiperbólica em seu nível de energia.

Na seção próxima seção, provaremos esta proposição. Mostraremos, a seguir, como ela implica o Teorema 3.1.1.

Demonstração de Teorema 3.1.1: A equação diferencial $x^{\prime \prime}=u_{\epsilon}(t) x$ depende diferenciavelmente do parâmetro $\epsilon$. Segue do Teorema da dependência de equações diferenciais de parâmetros (ver [33]), que as soluções $x=x(t, \epsilon)$ dependem diferenciavelmente do parâmetro $\epsilon$. Em particular, a função $\Phi=$ $\Phi(t, \epsilon)$ depende continuamente do parâmetro $\epsilon$. Calculemos, então, esta função em $\epsilon=0$. Nessa situação, temos a equação:

$$
x^{\prime \prime}=-\alpha^{2} x
$$

Pela definição das funções $x_{1}=x_{1}(t)$ e $x_{2}=x_{2}(t)$, temos: 


$$
x_{1}(t, 0)=\cos \alpha T \text { e } x_{2}(t, 0)=\frac{1}{\alpha} \sin \alpha T
$$

Logo, $\Phi_{0}(t)=\cos \alpha t$. Assim, $\Phi_{0}(T)=\cos \alpha T$. Pela hipótese do Teorema 1.1, temos que $\left(\Phi_{0}(T)\right)^{2} \in(0,1)$. Como a função $\Phi=\Phi_{\epsilon}(t)$ depende continuamente do parâmetro $\epsilon$, existe $\epsilon_{0}>0$, tal que se $\epsilon \in\left(-\epsilon_{0}, \epsilon_{0}\right)$, então $\left(\Phi_{\epsilon}(T)\right)^{2} \in(0,1)$. Por meio da Proposição 3.1.1, concluímos que a órbita de $z_{0}$ é elíptica em seu nível de energia.

Na próxima seção, provaremos a Proposição 3.1.1.

\subsection{Demonstração da Proposição 3.1.1}

O objetivo deta seção é provar a Proposição 3.1.1. Para isso, baseados no Lema 2.3.1, iremos estudar os autovalores da derivada do fluxo $d_{z_{0}} \phi_{T}^{H_{\epsilon}}$ : $T_{z_{0}} \mathbb{R}^{4} \longrightarrow T_{z_{0}} \mathbb{R}^{4}$. Seguiremos por partes.

1. O Polinômio Característico de $d_{z_{0}} \phi_{T}^{H_{\epsilon}}$.

O fluxo de $X_{H_{\epsilon}}$ tem a seguinte forma ${ }^{2}$ :

$$
\phi_{t}^{H_{\epsilon}}(z)=\left(X_{1}(t, z), X_{2}(t, z), Y_{1}(t, z), Y_{2}(t, z)\right)
$$

Segue que a derivada de $\phi_{t}^{H_{\epsilon}}$ pode ser, matricialmente, escrita por:

$$
d_{z} \phi_{t}^{H_{\epsilon}}=\left[\begin{array}{cccc}
\partial_{x_{1}} X_{1} & \partial_{x_{2}} X_{1} & \partial_{y_{1}} X_{1} & \partial_{y_{2}} X_{1} \\
\partial_{x_{1}} X_{2} & \partial_{x_{2}} X_{2} & \partial_{y_{1}} X_{2} & \partial_{y_{2}} X_{2} \\
\partial_{x_{1}} Y_{1} & \partial_{x_{2}} Y_{1} & \partial_{y_{1}} Y_{1} & \partial_{y_{2}} Y_{1} \\
\partial_{x_{1}} Y_{2} & \partial_{x_{2}} Y_{2} & \partial_{y_{1}} Y_{2} & \partial_{y_{2}} Y_{2}
\end{array}\right]
$$

Sendo que estas derivadas parciais estão sendo avaliadas em $(t, z)$. O polinômio característico de $d_{z_{0}} \phi_{T}^{H_{\epsilon}}$ é dado por:

$$
\operatorname{det}\left(\lambda I d-d_{z_{0}} \phi_{T}^{H_{\epsilon}}\right)=\operatorname{det}\left[\begin{array}{cccc}
\lambda-\partial_{x_{1}} X_{1} & -\partial_{x_{2}} X_{1} & -\partial_{y_{1}} X_{1} & -\partial_{y_{2}} X_{1} \\
-\partial_{x_{1}} X_{2} & \lambda-\partial_{x_{2}} X_{2} & -\partial_{y_{1}} X_{2} & -\partial_{y_{2}} X_{2} \\
-\partial_{x_{1}} Y_{1} & -\partial_{x_{2}} Y_{1} & \lambda-\partial_{y_{1}} Y_{1} & -\partial_{y_{2}} Y_{1} \\
-\partial_{x_{1}} Y_{2} & -\partial_{x_{2}} Y_{2} & -\partial_{y_{1}} Y_{2} & \lambda-\partial_{y_{2}} Y_{2}
\end{array}\right]
$$

\footnotetext{
${ }^{2}$ Por um momento, omitiremos a dependência do parâmetro $\epsilon$.
} 
Por meio de propriedades da função determinante (vista como função de seus vetores linha ou coluna), podemos escrever (trocando-se coluna 2 por coluna 3$)$ :

$$
\begin{gathered}
\operatorname{det}\left(\lambda I d-d_{z_{0}} \phi_{T}^{H_{\epsilon}}\right)=-\operatorname{det}\left[\begin{array}{cccc}
\lambda-\partial_{x_{1}} X_{1} & -\partial_{y_{1}} X_{1} & -\partial_{x_{2}} X_{1} & -\partial_{y_{2}} X_{1} \\
-\partial_{x_{1}} X_{2} & -\partial_{y_{1}} X_{2} & \lambda-\partial_{x_{2}} X_{2} & -\partial_{y_{2}} X_{2} \\
-\partial_{x_{1}} Y_{1} & \lambda-\partial_{y_{1}} Y_{1} & -\partial_{x_{2}} Y_{1} & -\partial_{y_{2}} Y_{1} \\
-\partial_{x_{1}} Y_{2} & -\partial_{y_{1}} Y_{2} & -\partial_{x_{2}} Y_{2} & \lambda-\partial_{y_{2}} Y_{2}
\end{array}\right] \\
=\operatorname{det}\left[\begin{array}{cccc}
\lambda-\partial_{x_{1}} X_{1} & -\partial_{y_{1}} X_{1} & -\partial_{x_{2}} X_{1} & -\partial_{y_{2}} X_{1} \\
-\partial_{x_{1}} Y_{1} & \lambda-\partial_{y_{1}} Y_{1} & -\partial_{x_{2}} Y_{1} & -\partial_{y_{2}} Y_{1} \\
-\partial_{x_{1}} X_{2} & -\partial_{y_{1}} X_{2} & \lambda-\partial_{x_{2}} X_{2} & -\partial_{y_{2}} X_{2} \\
-\partial_{x_{1}} Y_{2} & -\partial_{y_{1}} Y_{2} & -\partial_{x_{2}} Y_{2} & \lambda-\partial_{y_{2}} Y_{2}
\end{array}\right]
\end{gathered}
$$

Onde a última igualdade foi obtida trocando-se a linha 2 pela linha 3 . Essa matriz $4 \times 4$ pode ser representada, da forma usual, por blocos de matrizes $2 \times 2$. Dessa forma, podemos escrever:

$$
\operatorname{det}\left(\lambda I d-d_{z_{0}} \phi_{T}^{H_{\epsilon}}\right)=\operatorname{det}\left[\begin{array}{cc}
B_{1} & -B_{2} \\
-B_{3} & B_{4}
\end{array}\right]
$$

Diante dessa representação, temos:

Lema 3.2.1 A matriz $B_{2}$ é nula.

Demonstração: A matriz $B_{2}$ é dada por:

$$
B_{2}=\left[\begin{array}{cc}
\partial_{x_{2}} X_{1}\left(T, z_{0}\right) & \partial_{y_{2}} X_{1}\left(T, z_{0}\right) \\
\partial_{x_{2}} Y_{1}\left(T, z_{0}\right) & \partial_{y_{2}} Y_{1}\left(T, z_{0}\right)
\end{array}\right]
$$

Observemos que esta matriz corresponde a derivada da aplicação:

$$
\left(x_{2}, y_{2}\right) \mapsto\left(X_{1}\left(T, 0, x_{2}, 0, y_{2}\right), Y_{1}\left(T, 0, x_{2}, 0, y_{2}\right)\right)
$$

Avaliada em $\left(x_{2}, y_{2}\right)=\left(0, y_{2}^{E}\right)$. Como a subvariedade $M_{1}$, definida na seção 1, é invariante pelo fluxo de $X_{H_{\epsilon}}$, segue que esta aplicação é identicamente $(0,0)$. Portanto, $B_{2}=0_{2 \times 2}$. 
Com isso, obtemos os dois próximos corolários.

Corolário 3.2.1 O polinômio característico de $d_{z_{0}} \phi_{T}^{H_{\epsilon}}$ é dado por:

$$
\operatorname{det}\left(\lambda I d-d_{z_{0}} \phi_{T}^{H_{\epsilon}}\right)=\operatorname{det}\left(B_{1}\right) \operatorname{det}\left(B_{4}\right)
$$

Demonstração: Imediato a partir do lema anterior e da representação por blocos de matrizes $2 \times 2$ (igualdade 3.3).

Corolário 3.2.2 Os autovalores da seguinte matriz:

$$
\Delta=\left[\begin{array}{ll}
\partial_{x_{1}} X_{1}\left(T, z_{0}\right) & \partial_{y_{1}} X_{1}\left(T, z_{0}\right) \\
\partial_{x_{1}} Y_{1}\left(T, z_{0}\right) & \partial_{y_{1}} Y_{1}\left(T, z_{0}\right)
\end{array}\right]
$$

são autovalores de $d_{z_{0}} \phi_{T}^{H_{\epsilon}}: T_{z_{0}} \mathbb{R}^{4} \longrightarrow T_{z_{0}} \mathbb{R}^{4}$.

Demonstração: Devemos observar que os autovalores de $\Delta$ correspondem às raízes da equação $\operatorname{det}\left(B_{1}\right)=0$.

Observação 3.2.1 A ideia, na sequência, será analisar os autovalores dessa matriz $\Delta$, tendo em vista que eles são autovalores de $d_{z_{0}} \phi_{T}^{H_{\epsilon}}$ e que, pelo Lema 2.3.1, os autovalores de $d_{z_{0}} \phi_{T}^{H_{\epsilon}}$ são da forma $\left\{\lambda, \lambda^{-1}, 1,1\right\}$, onde $\left\{\lambda, \lambda^{-1}\right\}$ são autovalores da correspondente derivada da aplicação de Poincaré, no nível de energia. A partir destes, podemos classificar a órbita periódica sobre o nível de energia.

2. A Matriz $\Delta$.

Observemos que a matriz $\Delta$ corresponde a derivada da aplicação:

$$
\left(x_{1}, y_{1}\right) \mapsto\left(X_{1}\left(T, x_{1}, 0, y_{1}, y_{2}^{E}\right), Y_{1}\left(T, x_{1}, 0, y_{1}, y_{2}^{E}\right)\right)
$$

avaliada em $\left(x_{1}, y_{1}\right)=(0,0)$. Definimos as seguintes curvas auxiliares:

$$
z_{1}(t)=\frac{\partial \phi_{t}^{H_{\epsilon}}}{\partial x_{1}}\left(z_{0}\right) \text { e } z_{2}(t)=\frac{\partial \phi_{t}^{H_{\epsilon}}}{\partial y_{1}}\left(z_{0}\right)
$$

Então, definindo a matriz $A_{\epsilon}(t)=d X_{H_{\epsilon}}\left(\phi_{t}^{H_{\epsilon}}\left(z_{0}\right)\right)$, podemos caracterizar essas curvas e a matriz $\Delta$ por:

Lema 3.2.2 Pela definição das curvas $z_{1}$ e $z_{2}$, temos:

- $z_{1}$ e $z_{2}$ são soluções de: 


$$
\left\{\begin{array} { l } 
{ z _ { 1 } ^ { \prime } = A _ { \epsilon } ( t ) z _ { 1 } } \\
{ z _ { 1 } ( 0 ) = ( 1 , 0 , 0 , 0 ) }
\end{array} \quad e \left\{\begin{array}{l}
z_{2}^{\prime}=A_{\epsilon}(t) z_{2} \\
z_{2}(0)=(0,0,1,0)
\end{array}\right.\right.
$$

- Indicando por $\pi_{1}: \mathbb{R}^{4} \longrightarrow \mathbb{R}^{2}, \pi_{1}\left(x_{1}, x_{2}, y_{1}, y_{2}\right)=\left(x_{1}, y_{1}\right)$, a primeira coluna da matriz $\Delta$ é dada pelo vetor $\pi_{1}\left(z_{1}(T)\right)$ e a segunda coluna é dada pelo vetor $\pi_{1}\left(z_{2}(T)\right)$.

Demonstração: O primeiro item segue de propriedades da derivada da aplicação fluxo de uma equação diferencial ordinária (ver [33]) e o segundo segue da definição da matriz $\Delta$.

Devido a caracterização da matriz $\Delta$, por meio das soluções de $z^{\prime}=$ $A_{\epsilon}(t) z$, vamos analisar melhor esta equação. Observemos que, pela definição de $A_{\epsilon}$ e como a trajetória de $z_{0}$ é da forma $\phi_{t}^{H_{\epsilon}}\left(z_{0}\right)=\left(0, x_{2}(t), 0, y_{2}(t)\right)$, podemos escrever:

$$
A_{\epsilon}(t)=\left[\begin{array}{cccc}
0 & 0 & 1 & 0 \\
0 & 0 & 0 & 1 \\
-\alpha^{2}+2 \epsilon x_{2}^{2}(t) & 0 & 0 & 0 \\
0 & \nu^{2}-3 b_{2} x_{2}^{2}(t) & 0 & 0
\end{array}\right]
$$

Assim, por meio de blocos de matrizes $2 \times 2$, a matriz $A_{\epsilon}$ se escreve como:

$$
A_{\epsilon}(t)=\left[\begin{array}{cc}
0 & I d \\
U_{\epsilon}(t) & 0
\end{array}\right], \text { Onde } U_{\epsilon}(t)=\left[\begin{array}{cc}
u_{\epsilon}(t) & 0 \\
0 & v(t)
\end{array}\right]
$$

Com $u_{\epsilon}(t) \doteq-\alpha^{2}+2 \epsilon x_{2}^{2}(t)$ e $v(t) \doteq \nu^{2}-3 b_{2} x_{2}^{2}(t)$.

Suponhamos $z(t)=(x(t), y(t)) \in \mathbb{R}^{2} \times \mathbb{R}^{2}$ (ou seja, as curvas $x$ e $y$ estão no $\left.\mathbb{R}^{2}\right)$ solução de $z^{\prime}=A_{\epsilon}(t) z$. Então:

$$
z^{\prime}=\left[\begin{array}{l}
x^{\prime} \\
y^{\prime}
\end{array}\right]=\left[\begin{array}{cc}
0 & I d \\
U_{\epsilon}(t) & 0
\end{array}\right] \cdot\left[\begin{array}{l}
x \\
y
\end{array}\right]=\left[\begin{array}{c}
y \\
U_{\epsilon}(t) x
\end{array}\right]
$$

Logo:

$$
\left\{\begin{array}{l}
x^{\prime}=y \\
y^{\prime}=U_{\epsilon}(t) x
\end{array}\right.
$$


Portanto, a equação se reduz a uma equação diferencial ordinária, em $\mathbb{R}^{2}$, de segunda ordem:

$$
x^{\prime \prime}=U_{\epsilon}(t) x
$$

Procedendo da mesma forma, escrevendo a solução desta equação como $x(t)=\left(x_{1}(t), x_{3}(t)\right) \in \mathbb{R} \times \mathbb{R}$, obtemos:

$$
x^{\prime}=\left[\begin{array}{l}
x_{1}^{\prime \prime} \\
x_{3}^{\prime \prime}
\end{array}\right]=\left[\begin{array}{cc}
u_{\epsilon}(t) & 0 \\
0 & v(t)
\end{array}\right] \cdot\left[\begin{array}{l}
x_{1} \\
x_{3}
\end{array}\right]=\left[\begin{array}{c}
u_{\epsilon}(t) x_{1} \\
v(t) x_{3}
\end{array}\right]
$$

Logo:

$$
\left\{\begin{array}{l}
x_{1}^{\prime \prime}=u_{\epsilon}(t) x_{1} \\
x_{3}^{\prime \prime}=v(t) x_{3}
\end{array}\right.
$$

Tendo em vista essas simplificações da equação $z^{\prime}=A_{\epsilon}(t) z$ e as condições iniciais no Lema 3.2.2, podemos concluir:

- A curva $z_{1}=z_{1}(t)$ tem a forma $z_{1}(t)=\left(x_{1}(t), x_{3}(t), x_{1}^{\prime}(t), x_{3}^{\prime}(t)\right)$, onde:

$$
\left\{\begin{array}{l}
x_{1}^{\prime \prime}=u_{\epsilon}(t) x_{1} \\
x_{1}(0)=1 \\
x_{1}^{\prime}(0)=0
\end{array}\right.
$$

- A curva $z_{2}=z_{2}(t)$ tem a forma $z_{2}(t)=\left(x_{1}(t), x_{3}(t), x_{1}^{\prime}(t), x_{3}^{\prime}(t)\right)$, onde:

$$
\left\{\begin{array}{l}
x_{1}^{\prime \prime}=u_{\epsilon}(t) x_{1} \\
x_{1}(0)=0 \\
x_{1}^{\prime}(0)=1
\end{array}\right.
$$

Baseado nessas informações, podemos representar a matriz $\Delta$ da seguinte forma:

Proposição 3.2.1 Seja $M=M(t)$ a matriz dada por:

$$
M(t)=\left[\begin{array}{ll}
x_{1}(t) & x_{3}(t) \\
x_{1}^{\prime}(t) & x_{3}^{\prime}(t)
\end{array}\right]
$$

Onde: 


$$
\left\{\begin{array} { l } 
{ x _ { 1 } ^ { \prime \prime } = u _ { \epsilon } ( t ) x _ { 1 } } \\
{ x _ { 1 } ( 0 ) = 1 ; } \\
{ x _ { 1 } ^ { \prime } ( 0 ) = 0 ; }
\end{array} \quad e \left\{\begin{array}{l}
x_{3}^{\prime \prime}=u_{\epsilon}(t) x_{3} \\
x_{3}(0)=0 ; \\
x_{3}^{\prime}(0)=1 ;
\end{array}\right.\right.
$$

Então: $\Delta=M(T)$.

Demonstração: Segue da análise feita acima e do Lema 3.2.2.

Essa caracterização da matriz $\Delta$ nos permite escrever, para o seu polinômio característico:

Corolário 3.2.3 O polinômio característico da matriz $\Delta$ tem a seguinte forma:

$$
p_{\Delta}(\lambda)=\lambda^{2}-2 \Phi \lambda+1, \text { onde: } \Phi=\frac{x_{1}(T)+x_{3}^{\prime}(T)}{2} .
$$

Demonstração: Observemos que a matriz $M=M(t)$ é matriz fundamental da seguinte equação:

$$
X^{\prime}=\left[\begin{array}{cc}
0 & 1 \\
u_{\epsilon}(t) & 0
\end{array}\right] X
$$

Logo, pela Fórmula de Liouville (ver [33]), o determinante de $M(t)$ é dado por:

$$
\operatorname{det}(M(t))=\operatorname{det}(M(0)) e^{0}=\operatorname{det}(M(0))=1, \forall t \in \mathbb{R}
$$

Como $\Delta=M(T)$, o polinômio característico de $\Delta$ tem a seguinte forma:

$$
p_{\Delta}(\lambda)=\lambda^{2}-2 \Phi \lambda+1 \text {, onde: } 2 \Phi \text { é o traço de } M(T) \text {. }
$$

Segue o resultado.

Observação 3.2.2 Convém ressaltar que esse número $\Phi$ pode ser visto como função de $T$ (período da órbita) e do parâmetro $\epsilon$. De fato, as soluções $x_{1}=x_{1}(t)$ e $x_{3}=x_{3}(t)$ dependem do parâmetro $\epsilon$. Assim:

$$
\Phi=\frac{x_{1}(T, \epsilon)+x_{3}^{\prime}(T, \epsilon)}{2}
$$


Assim, escreveremos $\Phi=\Phi_{\epsilon}(T)$ para representar este número, da forma como ele aparece no enunciado da Proposição 3.1.1.

Diante dessas propriedades, vamos demonstrar a Proposição 3.1.1.

Demonstração da Proposição 3.1.1: Suponhamos $\lambda_{1}$ e $\lambda_{2}$ autovalores de $\Delta$. Então:

$$
\lambda_{1}=\Phi_{\epsilon}(T)+\sqrt{\Phi_{\epsilon}^{2}(T)-1} \text { e } \lambda_{2}=\Phi_{\epsilon}(T)-\sqrt{\Phi_{\epsilon}^{2}(T)-1}
$$

Temos: $\lambda_{1} \cdot \lambda_{2}=1$. Consideremos as situações:

- $\Phi_{\epsilon}^{2}(T) \in(0,1)$. Nesse caso, $\lambda_{1}$ e $\lambda_{2}$ têm parte imaginária não nula. Segue que $\lambda_{2}=\overline{\lambda_{1}}$. Assim, $\left|\lambda_{1}\right|,\left|\lambda_{2}\right|=1$. Obtemos que:

$$
\lambda_{1}, \lambda_{2} \in\{z \in \mathbb{C}:|z|=1\} \backslash\{-1,1\} .
$$

Como os autovalores de $\Delta$ são autovalores de $d_{z_{0}} \phi_{T}^{H_{\epsilon}}$ (Corolário 3.2.2) e tendo em vista o Lema 2.3.1, concluímos que $\lambda_{1}$ e $\lambda_{2}$ são autovalores da Transformação de Poincaré, sobre o nível de energia, da órbita de $z_{0}$. Segue que a órbita é elíptica em seu nível de energia.

- $\Phi_{\epsilon}^{2}(T)>1$. Nesse caso, $\lambda_{1}$ e $\lambda_{2}$ são reais, com módulos diferentes de 1 . Por um argumento análogo ao caso anterior, concluímos que a órbita de $z_{0}$ é hiperbólica no seu nivel de energia.

Concluímos assim a demonstração da Proposição 3.1.1.

Uma vez provado o Teorema 3.1.1, indicaremos, a seguir, as principais etapas para se concluir a demonstração do Teorema 1.1.1.

Demonstração do Teorema 1.1.1: Pelo Teorema 3.1.1, para completarmos a demonstração do Teorema 1.1.1, resta a análise da órbita hiperbólica $\Gamma_{E}^{\prime}$. Definimos a órbita $\Gamma_{E}^{\prime}$ como sendo a órbita no plano invariante $M_{2}$, com energia $E$ no sistema hamiltoniano bidimensional $X_{H_{1}}$. Procedendo de forma análoga ao que foi feito em relação a órbita elíptica, para $|\epsilon|$ pequeno, termos que $\Gamma_{E}^{\prime}$ é hiperbólica em seu nível de energia. As órbitas $\Gamma_{E}$ e $\Gamma_{E}^{\prime}$ estão linkadas no nível de energia $H_{\epsilon}^{-1}(E)$.

\subsection{Construção da Transformação de Poin- caré}

Nesta seção, construiremos a Transformação de Poincaré para a análise da dinâmica próxima a órbita periódica elíptica $\Gamma_{E}$, no nível de energia. Analisando a dinâmica da aplicação de Poincaré, para o caso $\epsilon=0$, colocaremos, 
na sequência, algumas questões que irão nos conduzir às demonstrações dos Teoremas 1.1.2 e 1.1.3.

Mostramos, então, que todo dado $E>0$, existe $\epsilon_{0}=\epsilon_{0}(E)>0$, tal que, quando $|\epsilon|<\epsilon_{0}$, a órbita $\Gamma_{E}$, construída no Lema 1.1.1, é elíptica no nível de energia. Na sequência, construiremos uma seção transversal para o estudo da Transformação de Poincaré, restrita ao nível de energia. Para isso, consideremos o seguinte conjunto:

$$
\Sigma=\left\{\left(x_{1}, x_{2}, y_{1}, y_{2}\right) \in \mathbb{R}^{4}: x_{2}=0\right\} .
$$

Vamos decompor este conjunto, considerando os seguintes subconjuntos:

$$
\begin{aligned}
& \Sigma^{+}=\left\{\left(x_{1}, x_{2}, y_{1}, y_{2}\right) \in \mathbb{R}^{4}: x_{2}=0 \text { e } y_{2}>0\right\} \\
& \Sigma^{0}=\left\{\left(x_{1}, x_{2}, y_{1}, y_{2}\right) \in \mathbb{R}^{4}: x_{2}=0 \text { e } y_{2}=0\right\} \\
& \Sigma^{-}=\left\{\left(x_{1}, x_{2}, y_{1}, y_{2}\right) \in \mathbb{R}^{4}: x_{2}=0 \text { e } y_{2}<0\right\}
\end{aligned}
$$

O conjunto $\Sigma^{0}=M_{2}$ (conjunto introduzido anteriormente), que é uma subvariedade invariante pelo fluxo de $X_{H_{\epsilon}}$. Os conjunto $\Sigma^{+}$e $\Sigma^{-}$são seções transversais a órbita $\Gamma_{E}$ (seções transversais em $\mathbb{R}^{4}$ ). Restringindo essas seções transversais ao nível de energia, obtemos seções sobre o nível de energia. Tendo em vista isso, definimos os seguintes conjuntos:

$$
\Sigma_{E}=\Sigma \cap H_{\epsilon}^{-1}(E) \text { e } \Sigma_{E}^{+,-, 0}=\Sigma^{+,-, 0} \cap H_{\epsilon}^{-1}(E) .
$$

Segue que o conjunto $\Sigma_{E}$ pode ser escrito como:

$$
\Sigma_{E}=\Sigma_{E}^{+} \cup \Sigma_{E}^{0} \cup \Sigma_{E}^{-} .
$$

Assim, $\Sigma_{E}^{+}$e $\Sigma_{E}^{-}$são seções transversais para a órbita $\Gamma_{E}$, sobre o nível de energia, e $\Sigma_{E}^{0}$ é uma órbita periódica de $X_{H_{\epsilon}}$, com energia $E$ (esta órbita corresponde a uma órbita periódica do sistema hamiltoniano bidimensional $X_{H_{1}}$, com energia $E$ ).

Notemos que se $\left(x_{1}, x_{2}, y_{1}, y_{2}\right) \in \Sigma$, então:

$$
x_{2}=0 \text { e } \frac{y_{1}^{2}+y_{2}^{2}}{2}+\frac{\alpha^{2} x_{1}^{2}}{2}+\frac{b_{1} x_{1}^{4}}{4}=E .
$$

Segue que $\Sigma_{E}$ é uma superfície, contida no nível de energia, que é difeomorfa a esfera bidimensional $\mathbb{S}^{2}$. Um esboço, desta situação, é indicado na próxima figura (Figura 3.3).

Os conjuntos $\Sigma_{E}^{+}$e $\Sigma_{E}^{-}$, seções transversais para a órbita $\Gamma_{E}$, correspondem aos hemisférios da esfera. O equador $\Sigma_{E}^{0}$ é uma órbita periódica, que corresponde ao bordo das seções transversais $\Sigma_{E}^{+}$e $\Sigma_{E}^{-}$. Para o estudo da Transformação de Poincaré da órbita $\Gamma_{E}$, restrito ao nível de energia, utilizaremos a seção $\Sigma_{E}^{+}$. Esta é uma, contida no nível de energia, que é a imagem da aplicação: 


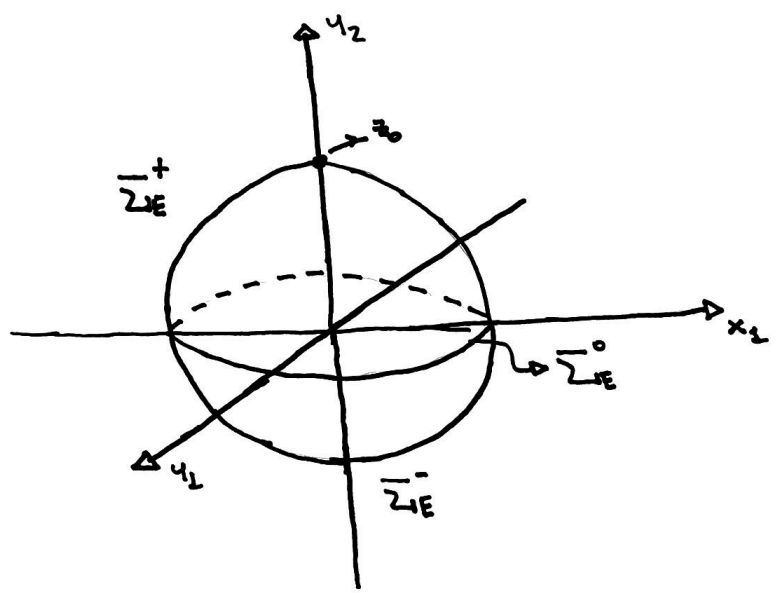

Figura 3.3: A esfera $\Sigma_{E}$.

$$
\begin{gathered}
\bar{z}: \Omega \longrightarrow \mathbb{R}^{4} \\
\left(x_{1}, y_{1}\right) \mapsto\left(x_{1}, 0, y_{1}, \bar{y}\left(x_{1}, y_{1}\right)\right) .
\end{gathered}
$$

Onde $\Omega$ é uma vizinhança de $(0,0) \in \mathbb{R}^{2}$, dada por:

$$
\Omega \doteq\left\{\left(x_{1}, y_{1}\right) \in \mathbb{R}^{2}: \frac{y_{1}^{2}}{2}+\frac{\alpha^{2} x_{1}^{2}}{2}+\frac{b_{1} x_{1}^{4}}{4}<E\right\} .
$$

E a aplicação $\bar{y}=\bar{y}\left(x_{1}, y_{1}\right)$ é dada por:

$$
\bar{y}\left(x_{1}, y_{1}\right)=\sqrt{2\left\{E-\left(\frac{y_{1}^{2}}{2}+\frac{\alpha^{2} x_{1}^{2}}{2}+\frac{b_{1} x_{1}^{4}}{4}\right)\right\}} .
$$

Observemos que $\Omega$ é um subconjunto do $\mathbb{R}^{2}$, que é invariante pelo fluxo do campo hamiltoniano bidimensional $X_{H_{1}}$. Este conjunto $\Omega$ pode ser decomposto como uma união de órbitas periódicas de $X_{H_{1}}$, órbitas com energia menor que $E$. A superfície $\Sigma_{E}^{+}$, seção transversal para a órbita $\Gamma_{E}$ de $X_{H_{\epsilon}}$, sobre o nível de energia $E$, não depende do parâmetro $\epsilon$.

Na sequência, analisaremos o comportamento da correspondente Transformação de Poincaré, segundo o parâmetro $\epsilon$ ser nulo ou não.

- Caso $\epsilon=0$. Vamos analisar a Transformação de Poincaré da órbita periódica $\Gamma_{E}$, de $X_{H_{\epsilon}}$, no caso em que $\epsilon=0$, e no caso em que $\alpha T \neq$ 
$2 k \pi, \forall k \in \mathbb{Z}$. Nessas condições, a Transformação de Poincaré está definida na seção $\Sigma_{E}^{+}$(definida na seção inteira), e seu estudo pode ser feito através de um simplectomorfismo $\psi_{0}: \Omega \rightarrow \Omega$, definido no sistema de coordenadas da seção $\Sigma_{E}^{+}$. Temos que $((0,0)) \in \Omega$ é um ponto fixo elíptico de $\psi_{0}$.

A dinâmica da aplicação de Poincaré $\psi_{0}$ é simples. O seu domínio $\Omega$ é folheado por curvas fechadas e invariantes por $\psi_{0}$, que são as mesmas curvas fechadas que aparecem na Figura 3 (por conservação de energia). Observemos que tais curvas correspondem a órbitas periódicas do campo bidimensional $X_{H_{1}}$. As órbitas de $\psi_{0}$ sobre cada uma dessas curvas ou são periódicas ou são densas sobre a curva. De fato, dado um ponto $\left(x_{1}, y_{1}\right) \in \Omega$, temos duas órbitas periódicas associadas: a órbita de $\left(x_{1}, y_{1}\right)$ por $X_{H_{1}}$ e a órbita de $\left(0, \bar{y}\left(x_{1}, y_{1}\right)\right)$ (onde $\bar{y}$ é definido em (3.5)) por $X_{H_{2}}$. Indicando por $T_{1}$ o período da órbita periódica de $X_{H_{1}}$ e por $T_{2}$ o período da órbita periódica de $X_{H_{2}}$, se $\frac{T_{2}}{T_{1}} \in \mathbb{Q}$, então a órbita de $\left(x_{1}, y_{1}\right)$ por $\psi_{0}$ é periódica; caso contrário, esta órbita será densa sobre a curva.

- Caso $\epsilon \neq 0$. Vamos supor $E>0$, com $\alpha T \neq 2 k \pi, \forall k \in \mathbb{Z}$, e $\Gamma_{E}$ a órbita dada pelo Lema 1.1.1. Fixemos $\epsilon$, com $|\epsilon|<\epsilon_{0}$, com $\epsilon \neq 0$, onde $\epsilon_{0}$ é dado pelo Teorema 1.1.1. Nessa situação, $\Gamma_{E}$ é órbita de $X_{H_{\epsilon}}$, elíptica em seu nível de energia. Ao contrário do caso $\epsilon=0$, não podemos garantir que a Transformação de Poincaré esteja definida na seção toda $\Sigma_{E}^{+}$. Indicaremos a correspondente Transformação de Poincaré por $\psi_{\epsilon}: \Omega_{\epsilon} \rightarrow \Omega_{\epsilon}$, onde $\Omega_{\epsilon} \subseteq \Omega$ é uma vizinhança de $(0,0)$, sendo este um ponto fixo elíptico.

\subsection{Algumas Questões}

Pela descrição feita acima, temos que $\psi_{0}: \Omega \rightarrow \Omega$ é um simplectomorfismo suave, cuja origem é um ponto fixo elíptico. O domínio $\Omega$ é folheado por curvas fechadas invariantes, sobre as quais a dinâmica de $\psi_{0}$ é, relativamente, simples de descrever. Por outro lado, temos $\psi_{\epsilon}: \Omega_{\epsilon} \rightarrow \Omega_{\epsilon}, \epsilon \neq 0$, que são simplectomorfismos cuja origem também é um ponto fixo elíptico. Iremos analisar a família de aplicações $\psi_{\epsilon}$ como uma perturbação da aplicação $\psi_{0}$. Gostaríamos de analisar como as propriedades descritas acima para $\psi_{0}$ (domínio folheado por curvas fechadas invariantes) se comportam diante dessa perturbação. $\mathrm{Ou}$ seja:

- Alguma dessas curvas fechadas invariantes persiste para a aplicação $\psi_{\epsilon}$, $\operatorname{com} \epsilon \neq 0$ ? 
- Que tipo de dinâmica irá surgir para $\epsilon \neq 0$ ? Se as curvas forem destruídas, que tipo de estrutura surgirá no sistema?

Observemos que para o caso $\epsilon=0$, uma vizinhança da órbita $\Gamma_{E}$, no nível de energia $E$, é folheada por toros bidimensinais invariantes. Portanto, questionar a persistência das curvas invariantes de $\psi_{0}$ é questionar a persistência desses toros invariantes para $\epsilon \neq 0$. No próximo capítulo, analisaremos uma mudança de coordenadas que nos permitirá colocar essas questões de uma forma "mais tratável". 


\section{Capítulo 4}

\section{Mudança Conveniente de Coordenadas.}

O Teorema 1.1.1 nos dá uma condição para que a órbita $\Gamma_{E}$, de $X_{H_{\epsilon}}$, seja elíptica no nível de energia, para $|\epsilon|$ pequeno. Nesse caso, o estudo da correspondente Transformação de Poincaré é feito através de um difeomorfismo, que preserva área, definido numa vizinhança de $(0,0) \in \mathbb{R}^{2}$, cuja origem é um ponto fixo elíptico. Neste capítulo, iremos estudar uma mudança conveniente de coordenadas para essa situação, baseada na Forma Normal de Birkhoff, e veremos como ficam, nessas novas coordenadas, algumas das questões colocadas nos Teoremas 1.1.2 e 1.1.3.

\subsection{A Forma Normal de Birkhoff}

Suponhamos $V_{0} \subseteq \mathbb{R}^{2}$, vizinhança de $(0,0) \in \mathbb{R}^{2}$, e $f: V_{0} \longrightarrow V_{0}$ um difeomorfismo de classe $C^{\infty}$, que preserva área e cuja origem é um ponto fixo, isto é, $f(0,0)=(0,0)$. Sejam $\lambda_{1}, \lambda_{2} \in \mathbb{C}$ autovalores de $d f(0,0)$. Como $f$ preserva área, temos $\lambda_{1} \cdot \lambda_{2}=1$. Ou seja, os autovalores de $d f(0,0)$ são da forma $\lambda, \frac{1}{\lambda}$, com $\lambda \in \mathbb{C}$.

Se $\lambda \in \mathbb{R} \backslash\{1,-1\}$, então a origem é um ponto fixo hiperbólico de $f$. Nesse caso, o teorema de Hartman-Grobman nos diz que, localmente, próximo a origem, as órbitas de $f$ se comportam como as órbitas de $d f(0,0)$, o sistema linearizado de $f$. Portanto, a derivada de $f$, na origem $(0,0) \in \mathbb{R}^{2}$, determina o comportamento da dinâmica próxima ao ponto fixo.

Uma outra possibilidade é que o número $\lambda \in \mathbb{C}$ seja da forma $\lambda=e^{i \alpha}$. Nesse caso, se $\lambda^{2} \neq 1$, então o ponto fixo é um ponto fixo elíptico. Nessa situação, ao contrário do que ocorre no caso hiperbólico, a primeira derivada é insuficiente para descrever o comportamento dinâmico local e precisamos 


\section{CAPÍTULO 4. MUDANÇA CONVENIENTE DE COORDENADAS.}

recorrer a derivadas de ordem superior. É possível associar uma Forma Normal a $f$, próximo ao ponto fixo, dentro de certas condições, que é a chamada Forma Normal de Birkhoff (ver [23] e [14]). Tal forma normal depende dos jatos de ordem superior de $f$ em $(0,0)$ e é descrita abaixo.

Teorema 4.1.1 (Forma Normal de Birkhof) Suponhamos $f:\left(\mathbb{R}^{2}, 0\right) \longrightarrow$ $\left(\mathbb{R}^{2}, 0\right)$ um simplectomorfismo suave, cuja origem é um ponto fixo e $\lambda=e^{2 \pi i \alpha}$ $e \bar{\lambda}$ são os autovalores de df(0). É satisfeita a seguinte hipótese sobre o autovalor $\lambda$ :

$$
\exists q \in \mathbb{N} \text { tal que } \lambda^{n} \neq 1, \forall n \in\{1,2, \ldots, q\}
$$

Então existe um difeomorfismo $h, C^{\infty}$, que preserva área, tal que em coordenadas complexas, $z=x+i y, \bar{z}=x-i y$, tenhamos:

$$
\begin{gathered}
h \circ f \circ h^{-1}(z)=\lambda z e^{2 \pi i P(z \bar{z})}+o\left(|z|^{q-1}\right), \\
\text { onde: } P(x)=\sum_{i=1}^{m} c_{i} x^{i} \text {, sendo que } 2 m+1<q .
\end{gathered}
$$

Cada coeficiente $c_{i}$ do polinômio $P$, que aparece na expressão da forma normal, é chamado invariante de Birkhoff.

Em relação a este teorema, temos as seguintes observações:

Observação 4.1.1 Notemos que:

1. Escrevendo a expressão obtida na Forma Normal de Birkhoff em coordenadas polares $(\theta, r)$, conseguimos uma aplicação $\tilde{f}$ da forma:

$$
\tilde{f}=(\theta, r) \mapsto\left(\theta+\alpha+P\left(r^{2}\right), r\right)+o\left(|r|^{q-1}\right)=(\Theta, R) .
$$

2. Segue que diminuindo $U$, se necessário, existe um difeomorfismo $G_{1}$ : $U \backslash\{(0,0)\} \longrightarrow \mathbb{S}^{1} \times(0, b)$, para algum $b>0$, tal que $G_{1} \circ f=\tilde{f} \circ G_{1}$, onde $\tilde{f}$ é dada acima. É importante ressaltar que vale:

$$
G_{1}^{*}(r d \theta \wedge d r)=d x \wedge d y
$$

3. Sendo $\tilde{f}(\theta, r)=(\Theta, R)$ e se algum invariante de Birkhoff for não nulo, então:

$$
\frac{\partial \Theta}{\partial r}(\theta, r) \neq 0, \text { para } r>0 \text { suficientemente pequeno. }
$$


Ou seja, a aplicação $\tilde{f}$ satisfaz uma condição twist.

4. Seria interessante que $\tilde{f}: \mathbb{S}^{1} \times(0, b) \longrightarrow \mathbb{S}^{1} \times(0, b)$ preservasse a 2forma simplética $d \theta \wedge d r$. Notemos que ela preserva $r d \theta \wedge d r$. Para contornar esse problema, faremos uma outra mudança de coordenadas. Definimos:

$$
G_{2}: \mathbb{S}^{1} \times(0, b) \longrightarrow \mathbb{S}^{1} \times(0, a), G_{2}(\theta, r)=\left(\theta, \frac{r^{2}}{2}\right)=(\Theta, R)
$$

Observemos $^{1}$ que $G_{2}^{*}(d \Theta \wedge d R)=r d \theta \wedge d r$.

Seja $\bar{f}=G_{2} \circ \tilde{f} \circ G_{2}^{-1}$.

5. Observemos que $\bar{f}$ satisfaz a condição twist se, e somente se, $\tilde{f}$ satisfaz.

6. Seja $G: U \backslash\{(0,0)\} \longrightarrow \mathbb{S}^{1} \times(0, a), G=G_{2} \circ G_{1}$. Se alguma invariante de Birkhoff for não nulo, então $G$ conjuga $f$ em $U$ a $\bar{f} \mathrm{em}$ $\mathbb{S}^{1} \times(0, a)$, com $\bar{f}$ satisfazendo uma condição twist. Devemos notar que: $G_{2}^{*}(R d \Theta)=\frac{r^{2}}{2} d \theta$. Além disso, vale que se $\lambda=R d \Theta$, obtemos, portanto ${ }^{2}, G^{*} \lambda=\mu$, onde:

$$
\mu=\frac{x}{2} d y-\frac{y}{2} d x
$$

Observemos que $f^{*} \mu-\mu$ é exata, pois $f$ preserva área e $U$ é contrátil. Logo, $\bar{f}^{*} \lambda-\lambda$ é exata em $\mathbb{S}^{1} \times(0, a)$. Portanto, $G$ conjuga $f$ a uma aplicação twist simplética exata (se algum invariante for não nulo).

7. A ideia para essa segunda mudança de coordenadas aparece em [8] e, em dimensões maiores, em [Y].

Um importante resultado relacionado a uma aplicação $f: U \rightarrow U$, satisfazendo as condições do Teorema da Forma Normal de Birkhoff, é apresentado a seguir. O resultado se refere a estabilidade do ponto fixo em questão. É uma versão do resultado conhecido como Teorema do Twist de Moser (ver [23] para maiores detalhes).

Teorema 4.1.2 Seja $f: U \rightarrow U$ uma aplicação satisfazendo as condições do Teorema da Forma Normal de Birkhoff. Se o polinômio associado (dado no teorema anterior) for não nulo, então dado qualquer vizinhança $V \subseteq U$ de $(0,0) \in U$, existe uma curva fechada $\gamma$, contida em $V$, homotopicamente não

\footnotetext{
${ }^{1}$ Por abuso de notação, indicamos as coordenadas de $\mathbb{S}^{1} \times(0 ; a)$ por $(\Theta, R)$
}

${ }^{2} G_{1}^{*}\left(\frac{r^{2}}{2} d \theta\right)=\mu$ 
trivial em $V \backslash\{(0,0)\}$, tal que $f(\gamma)=\gamma$. Ou seja, se algum dos invariantes de Birkhoff, associado a forma normal, é não nulo, então exitem curvas fechadas invariantes por $f$, homotopicamente não triviais, arbitrariamente próxima ao ponto fixo.

Demonstração: $\operatorname{Ver}$ [23].

\subsection{Aplicando o Teorema 4.1.1 às Aplicações de Poincaré}

Vamos aplicar o Teorema 4.1.1 a Transformação de Poincaré de $\Gamma_{E}$, sobre o nível de energia. No nosso caso de interesse, apresentado na seção anterior, temos uma família de simpectomorfismos $\psi_{\epsilon}: \Omega_{\epsilon} \rightarrow \Omega_{\epsilon}$, onde $\Omega_{\epsilon}$ é uma pequena vizinhança da origem. Temos que a origem é um ponto fixo elíptico, sendo os autovalores, para o caso $\epsilon=0$, dados por $e^{i \alpha T}$ e $e^{-i \alpha T}$. Para que a Forma Normal de Birkhoff esteja bem definida para essa aplicação $\psi_{0}$, é suficiente impor a seguinte condição. Escrevendo $\lambda=e^{i \alpha T}$, suponhamos que:

$$
\lambda^{j} \neq 1, \text { para } j=1,2,3,4 .
$$

Nessas condições, o Teorema 4.1 .1 pode ser aplicado a Transformação $\psi_{0}: \Omega \rightarrow \Omega$. O parâmetro $q$, do enunciado do Teorema 4.1.1, pode ser tomado como $q=4$. Notemos ainda que essa condição é equivalente a:

$$
\cos j \alpha T+i \sin j \alpha T \neq 1, \forall j \in\{1,2,3,4\} .
$$

Ou seja, $j \alpha T \neq 2 k \pi, \forall k \in \mathbb{Z}$, onde $j=1,2,3,4$.

Baseado nisso definimos o seguinte conjunto de valores de energia, que aparece no enunciado dos Teoremas 1.1.2 e 1.1.3.

Notação: Consideremos $J \subseteq(0,+\infty)$ definido como:

$$
J \doteq\left\{E \in(0,+\infty): \alpha T \neq 2 k \pi, k \pi, \frac{k \pi}{2}, \frac{2 k \pi}{3}: k \in \mathbb{Z}\right\} .
$$

Com isso, obtemos o próximo lema.

Lema 4.2.1 Suponhamos $E \in J$. Então existe $\epsilon_{0}=\epsilon_{0}(E)>0$, tal que se $|\epsilon|<\epsilon_{0}$, então a Forma Normal de Birkhoff da aplicação $\psi_{\epsilon}: \Omega_{\epsilon} \rightarrow \Omega_{\epsilon}$ está bem definida.

Demonstração: Observemos que para $|\epsilon|$ suficientemente pequeno, (0,0) é um ponto fixo elíptico de $\psi_{\epsilon}$. Se $E \in J$, pela argumentação feita acima, a Forma Normal de Birkhoff está definida para $\psi_{0}$. Como os autovalores de $\psi_{\epsilon}$ variam continuamente com o parâmetro $\epsilon$, segue que diminuindo $\epsilon$, se necessário, temos que se $E \in J$, então a Forma Normal de Birkhoff de $\psi_{\epsilon}$ também esta definida. 
Em relação ao domínio das aplicações $\psi_{\epsilon}: \Omega_{\epsilon} \rightarrow \Omega_{\epsilon}$, faremos a seguinte consideração.

Observação 4.2.1 Como muitas das questões que faremos serão analisadas numa pequena vizinhança de $(0,0)$, iremos supor que as Transformações de Poincaré, $\psi_{\epsilon}$, estejam definidas num mesmo domínio, que indicaremos por $\Omega$, obtido diminuindo o domínio da aplicação $\psi_{0}$.

\subsection{Algumas Questões em coordenadas twist.}

Portanto, diante da condição $E \in J$ e da observação 4.2.1, a Forma Normal de Birkhoff fica bem definida para a família de aplicações $\psi_{\epsilon}: \Omega \rightarrow \Omega$, quando $\epsilon$ é suficientemente pequeno. Assim, temos uma boa caracterização de suas expressões, quando escritas em coordenadas polares. Tendo em vista as observações feitas após o Tereoma 4.1.1, seria interessante dizer se algum invariante de Birkhoff, dessas aplicações, é nulo ou não. Isso não é algo tão simples, pois, como foi comentado, eles dependem dos jatos de ordem superior da aplicação. No caso do primeiro invariante de Birkhoff, ele depende do jato de ordem 3. Suponhamos, além de $E \in J$, que algum dos invariantes de Birkhoff seja não nulo. Vamos analisar como ficariam as questões levantadas no capítulo anterior (última seção do capítulo anterior). Teremos, nessas condições, que $\psi_{\epsilon}$ é conjugada a uma aplicação twist simplética exata $\varphi_{\epsilon}$. Vimos que o domínio da aplicação $\psi_{0}$ é folheado por curvas fechadas invariantes. Isso, no contexto da teoria de aplicações twist, pode ser formulado para a aplicação correspondente $\varphi_{0}$, dizendo-se que $\varphi_{0}$ é uma aplicação twist integrável: seu domínio é folheado por curvas rotacionais invariantes, cuja dinâmica, sobre elas, é conjugada a uma rotação rígida. Dessa forma, colocaríamos as questões do capítulo anterior, dentro da teoria de aplicações twist, da seguinte maneira:

- Para $\epsilon \neq 0$, a aplicação twist $\varphi_{\epsilon}$ é integrável? Possui curvas rotacionais invariantes?

- Se não for integrável, que propriedades teríamos para $\varphi_{\epsilon}$ ?

O objetivo deste trabalho será mostrar que para $\epsilon \neq 0$, pequeno, surgem zonas de instabilidade "se acumulando em $r=0$ ", produzindo entropia topológica positiva para as aplicações $\varphi_{\epsilon}, \epsilon \neq 0$, pequeno. Se provarmos essas afirmações, teremos provado o Teorema 1.1.3.

Diante das colocações feitas nessa seção, é fundamental saber se algum dos invariantes de Birkhoff é não nulo. Analisaremos essa questão para o primeiro 


\section{CAPÍTULO 4. MUDANÇA CONVENIENTE DE COORDENADAS.}

invariante de Birkhoff, isto é, o coeficiente $c_{1}$ do polinômio apresentado no Teorema da Forma Normal de Birkhoff. Esse será o assunto do próximo capítulo. 


\section{Capítulo 5}

\section{O Primeiro Invariante de Birkhoff}

O objetivo deste capítulo é analisar o primeiro invariante de Birkhoff para a família de aplicações ${ }^{1} \psi_{\epsilon}: \Omega \rightarrow \Omega$. Nesse sentido, estabeleceremos uma fórmula para este invariante, correspondente ao caso $\epsilon=0$, como uma função do nível de energia. Além disso, neste capítulo, provaremos o Teorema 1.1.2.

\subsection{Fórmula para o Primeiro Invariante de Birkhoff.}

Apresentaremos uma expressão (uma fórmula) para o cálculo do primeiro invariante, baseado em [10].

Suponhamos $f: U \longrightarrow U$ difeomorfismo que preserva área, como no enunciado do teorema da forma normal, com $f(0,0)=(0,0)$, e seja $\mu$ autovalor de $d f(0)$, que será escrito como:

$$
\mu=\cos \gamma+i \sin \gamma=e^{i \gamma}
$$

A expansão em série de Taylor de $f$ em $(0,0) \in U$, nas variáveis $(x, y)$ nos dá:

$$
\begin{aligned}
& f(x, y)=\left(a_{10} x+a_{01} y+a_{20} x^{2}+\ldots+a_{03} x^{3}, b_{10} x+b_{01} y+b_{20} x^{2}+\ldots+b_{03} y^{3}\right)+ \\
& +o\left(\|(x, y)\|^{4}\right) .
\end{aligned}
$$

\footnotetext{
${ }^{1}$ Quando escrevemos $\psi_{\epsilon}: \Omega \rightarrow \Omega$, estamos assumindo a situação da observação 4.2.1.
} 
Em coordenadas complexas conjugadas, é possível escrever:

$$
f(z, \bar{z})=\mu\left(z+c_{20} z^{2}+c_{11} z \bar{z}+\ldots+c_{03} \bar{z}^{3}\right)+o\left(|z|^{4}\right) .
$$

Assumiremos que:

$$
\mu^{j} \neq 1,2,3,4
$$

Proposição 5.1.1 Nessas condições, o primeiro invariante de Birkhoff pode ser escrito em termos dos coeficientes da expansão de $f$ em coordenadas complexas conjugadas $(z, \bar{z})$ :

$$
\tau=\operatorname{Im}\left(c_{21}\right)+\frac{\sin \gamma}{\cos \gamma-1}\left(3\left|c_{20}\right|^{2}+\frac{2 \cos \gamma-1}{2 \cos \gamma+1}\left|c_{02}\right|^{2}\right),
$$

onde $\operatorname{Im}\left(c_{21}\right)$ denota a parte imaginária do coeficiente $c_{21}$.

Demonstração: Ver [22] e [10].

Os coeficientes da expansão em coordenadas complexas conjugadas dependem dos coeficientes $\left(a_{i, j}\right)_{i, j}$ e $\left(b_{i, j}\right)_{i, j}$ da expansão em variáveis reais $(x, y)$. Observemos que os coeficientes $\left(a_{i j}\right)$ e $\left(b_{i j}\right)$, para $i+j=1$, são os coeficientes da matriz $d f(0)$. Como $f$ preserva área:

$$
a_{10} b_{01}-a_{01} b_{10}=1 .
$$

Para o caso em que $a_{10}=b_{01}$, teremos $a_{10}^{2}-a_{01} b_{10}=1$ (que é a situação da aplicação $\left.\psi_{0}\right)$. Utilizando essas relações, mostra-se ${ }^{2}$ que:

- $\operatorname{Im}\left(c_{21}\right)=\frac{a_{10}}{8}\left(-a_{21}+3 \frac{b_{10}}{a_{10}} a_{03}-3 \frac{a_{01}}{b_{10}} b_{30}+b_{12}\right)-\frac{b_{10}}{8}\left(a_{12}-3 \frac{a_{01}}{b_{10}} a_{30}-\right.$ $\left.\frac{a_{01}}{b_{10}} b_{21}+3 b_{03}\right)$.

- $\left|c_{20}\right|^{2}=\frac{1}{16} \sqrt{\frac{-a_{01}}{b_{10}}}\left(\frac{b_{10}}{a_{01}} a_{02}+a_{20}+b_{11}\right)^{2}+\frac{1}{16} \sqrt{\frac{-b_{10}}{a_{01}}}\left(\frac{a_{01}}{b_{10}} b_{20}+b_{02}+a_{11}\right)^{2}$.

- $\left|c_{20}\right|^{2}=\frac{1}{16} \sqrt{\frac{-a_{01}}{b_{10}}}\left(\frac{b_{10}}{a_{01}} a_{02}+a_{20}-b_{11}\right)^{2}+\frac{1}{16} \sqrt{\frac{-b_{10}}{a_{01}}}\left(\frac{a_{01}}{b_{10}} b_{20}+b_{02}-a_{11}\right)^{2}$.

Essas igualdades e a proposição anterior mostram que é possível determinar o primeiro invariante de Birkhoff em termos dos coeficientes da expansão em série de Taylor nas variáveis $(x, y)$. Portanto, precisaremos determinar o jato de ordem 3 das aplicações $\psi_{\epsilon}: \Omega \rightarrow \Omega$, e a partir disso calcular o invariante de Birkhoff, utilizando a fórmula dada acima. É o que faremos na próxima seção.

${ }^{2} \operatorname{Ver}[10]$. 


\subsection{A Aplicação $\psi_{0}$ de uma forma conveni- ente.}

Objetivo, dessa seção, é escrever a aplicação $\psi_{0}: \Omega \rightarrow \Omega$ de uma forma que nos permita analisar o correspondente primeiro invariante de Birkhoff, segundo a fórmula estabelecida acima.

Estamos indicando as coordenadas da seção $\Sigma_{E}^{+}$por $(x, y)$ (ao contrário de $\left.\left(x_{1}, y_{1}\right)\right)$. No caso $\epsilon=0$, o sistema está desacoplado. Segue que o comportamento do sistema é determinado pelos sistemas hamiltonianos bidimensionais, $H_{1}$ e $H_{2}$, definidos em (3.1). Indicando $\phi=\phi_{t}^{H_{1}}$ o fluxo do sistema hamiltoniano bidimensional $X_{H_{1}}$ e $T_{0}=T_{0}(x, y)$ a função tempo de retorno, para o caso $\epsilon=0$, da aplicação de Poincaré, em termos das coordenadas $(x, y) \in \Omega$, da seção escolhida ${ }^{3}$. Conforme vem sendo feito, indicaremos período, da órbita periódica $\Gamma_{E}$, por $T$. Portanto, $T=T_{0}(0,0)$.

A aplicação $\psi_{0}: \Omega \rightarrow \Omega$ pode ser escrita da seguinte maneira:

$$
\psi_{0}(x, y)=\phi\left(T_{0}(x, y), x, y\right)
$$

Iremos escrever a função $T_{0}=T_{0}(x, y)$ de uma maneira conveniente. Seja $P_{2}:(0,+\infty) \longrightarrow \mathbb{R}$, a função que associa a cada nível de energia, $E_{2}>0$, o período da órbita do sistema Hamiltoniano $X_{H_{2}}$ com energia $E_{2}$. Se $t \mapsto$ $\left(\tilde{x}_{2}(t), \tilde{y}_{2}(t)\right)$ indica esta essa órbita, então temos:

$$
\frac{\tilde{y}^{2}(t)}{2}+V_{2}\left(\tilde{x}_{2}(t)\right)=E_{2}
$$

Conforme discutido em [3] e [19], por exemplo, a função $P_{2}=P_{2}\left(T_{2}\right)$ pode ser dada em termos da seguinte integral elíptica:

$$
P_{2}\left(E_{2}\right)=4 \int_{0}^{x\left(E_{2}\right)} \frac{d x_{2}}{\sqrt{2\left(E_{2}-V_{2}\left(x_{2}\right)\right)}} .
$$

Onde $x\left(E_{2}\right)$ representa a raiz real e positiva de $V_{2}\left(x_{2}\right)-E_{2}=0$. Além disso, essa função $P_{2}$ é diferenciável, como função da energia $E_{2}$.

Observemos que se $(x, y) \in \Omega$, a órbita correspondente do Sistema Hamiltoniano $X_{H_{2}}$ passará pelo ponto $(0, \bar{y}(x, y))$, onde $\bar{y}=\bar{y}(x, y)$ é dada pela equação (3.5). O tempo de retorno da órbita correspondente a $(x, y)$, na seção, é o período da órbita de $(0, \bar{y}(x, y))$ (pela construção da seção transversal). Notemos, ainda, que a energia dessa órbita no sistema hamiltoniano $X_{\mathrm{H}_{2}}$ é dada por:

\footnotetext{
${ }^{3}$ Conforme vem sendo destacado no texto, $T$ é período da órbita periódica $\Gamma_{E} ; T_{0}$ é uma função.
} 


$$
E_{2}=\frac{\bar{y}^{2}(x, y)}{2}
$$

Seguirá que vale:

$$
T_{0}(x, y)=P_{2}\left(\frac{\bar{y}^{2}(x, y)}{2}\right)=P_{2}\left(E-V_{1}(x)-\frac{y^{2}}{2}\right) .
$$

Sendo $E$ a energia da órbita periódica $\Gamma_{E}$. Em relação a função $T_{0}=$ $T_{0}(x, y)$, temos o próximo lema.

Lema 5.2.1 Valem as seguintes igualdades:

- $\frac{\partial^{2} T_{0}}{\partial x \partial y}(0,0)=\frac{\partial T_{0}}{\partial x}(0,0)=\frac{\partial T_{0}}{\partial y}(0,0)=0$;

- $\frac{\partial^{2} T_{0}}{\partial x^{2}}(0,0)=-\alpha^{2} P_{2}^{\prime}(E)$;

- $\frac{\partial^{2} T_{0}}{\partial y^{2}}(0,0)=-P_{2}^{\prime}(E)$;

Onde $E$ é a energia da órbita periódica $\Gamma_{E}$. Além disso, as derivadas de ordem 1 , em $(0,0)$, são nulas.

Demonstração: Segue da igualdade:

$$
T_{0}(x, y)=P_{2}\left(\frac{\bar{y}^{2}(x, y)}{2}\right)=P_{2}\left(E-V_{1}(x)-\frac{y^{2}}{2}\right) .
$$

Derivando, aplicando a regra da cadeia, e calculando em $(x, y)=(0,0)$.

Diante disso, obtemos uma forma conveniente de escrever $T_{0}=T_{0}(x, y)$.

Corolário 5.2.1 Podemos escrever:

$$
T_{0}(x, y)=T+e(x, y),
$$

onde $T=T_{0}(0,0)$ é o período da órbita periódica $\Gamma_{E}$ e a aplicação e $=e(x, y)$ tem a seguinte forma:

$$
e(x, y)=t_{20} x^{2}+t_{02} y^{2}+r_{e}(x, y)
$$

sendo a função $r_{e}=r_{e}(x, y)$ uma função diferenciável que se anula em $(x, y)=(0,0)$, assim como suas derivadas até ordem 2.

O nosso objetivo será analisar o jato de ordem 3 de $\psi_{0}$ em $(0,0)$, tendo em vista a discussão feita, no capítulo anterior, sobre a Forma Normal de Birkhoff. Indicaremos o jato de ordem 3 de $f$ em $(0,0)$ por $J^{3} f(x, y)$. Assim, as componentes de $J^{3} f(x, y)$ são polinômios homogêneos, em duas variáveis $x$ e $y$, de grau 3.

O próximo resultado fará uma descrição de $J^{3} \psi_{0}$. 
Proposição 5.2.1 Vale a seguinte igualdade:

$$
J^{3} \psi_{0}(x, y)=J^{3} \phi_{T}(x, y)+P(x, y),
$$

onde $P=P(x, y)$ é a aplicação polinomial dado por:

$$
P(x, y)=\left(t_{20} x^{2}+t_{02} y^{2}\right)\left(d_{(0,0)}\left(X_{H_{1}} \circ \phi_{T}\right)(x, y)\right) .
$$

A aplicação $\phi_{T}$ é o fluxo hamiltoniano do sistema bidimensional $X_{H_{1}}$ no instante $t=T$, onde $T$ é o período da órbita $\Gamma_{E}$.

Demonstração: Observemos que:

$$
\psi_{0}(x, y)=\phi(T+e(x, y), x, y)
$$

Mas em relação ao fluxo $\phi$, podemos escrever:

$$
\phi(T+t, x, y)=\phi_{T}(x, y)+t X_{H_{1}}\left(\phi_{T}(x, y)\right)+r(t, x, y) .
$$

Onde $r=r(t, x, y)$ se anula em $t=0$, assim como suas derivadas de ordem 1, em relação a variável $t$.

Fazendo $t=e(x, y)$, obtemos:

$$
\psi_{0}(x, y)=\phi_{T}(x, y)+e(x, y) X_{H_{1}}\left(\phi_{T}(x, y)\right)+r_{1}(x, y) .
$$

Onde $r_{1}(x, y)=r(e(x, y), x, y)$. A partir daqui, seguiremos por partes.

1. A função $r_{1}=r_{1}(x, y)$ não contribui com $J^{3} \psi_{0}(x, y)$, pois $r_{1}$ se anula em $(0,0)$, assim como suas derivadas até terceira ordem.

2. Analisemos o termo $X_{H_{1}}\left(\phi_{T}(x, y)\right)$. Podemos escrever:

$$
X_{H_{1}}\left(\phi_{T}(x, y)\right)=d_{(0,0)}\left(X_{H_{1}} \circ \phi_{T}\right)(x, y)+r_{2}(x, y),
$$

onde a função $r_{2}=r_{2}(x, y)$ se anula em $(x, y)=(0,0)$, assim como suas derivadas de ordem 1. Segue que:

$$
e(x, y) X_{H_{1}}\left(\phi_{T_{0}}(x, y)\right)=e(x, y) d_{(0,0)}\left(X_{H_{1}} \circ \phi_{T_{0}}\right)(x, y)+e(x, y) r_{2}(x, y) .
$$

Afirmação: O termo e $(x, y) r_{2}(x, y)$ não contribui com $J^{3} \psi_{0}(x, y)$, ou melhor, a função e $(x, y) r_{2}(x, y)$ se anula em $(0,0)$, assim como suas derivadas até terceira ordem.

Diante dessa afirmação, concluímos que o termo de e $(x, y) X_{H_{1}}\left(\phi_{T_{0}}(x, y)\right)$, que contribui com o $J^{3} \psi_{0}(x, y)$, é dado por:

$$
P(x, y)=\left(t_{20} x^{2}+t_{02} y^{2}\right)\left(d_{(0,0)}\left(X_{H_{1}} \circ \phi_{T_{0}}\right)(x, y)\right) .
$$


Pelo que foi destacado nos itens 1 e 2, concluímos que:

$$
J^{3} \psi_{0}(x, y)=J^{3} \phi_{T}(x, y)+P(x, y) .
$$

Concluímos, assim, a demonstração da proposição.

Observação 5.2.1 Devemos observar que esta proposição nos diz que o jato de ordem 3 de $\psi_{0}$ em $(0,0)$ coincide com o jato de ordem 3 da aplicação $\phi_{T}$, em $(0,0)$, a menos da aplicação polinomial, homogênea de grau $3, P=$ $P(x, y)$. Assim, o polinômio $P=P(x, y)$ detecta a influência do sistema hamiltoniano $X_{\mathrm{H}_{2}}$ no primeiro invariante de Birkhoff.

Vamos, agora, escrever a expressão "completa" da aplicação $P=P(x, y)$ obtido na proposição anterior. Temos:

$$
\begin{gathered}
d_{(0,0)}\left(X_{H_{1}} \circ \phi_{T}\right)(x, y)=\left[\begin{array}{cc}
0 & 1 \\
-\alpha^{2} & 0
\end{array}\right]\left[\begin{array}{cc}
\cos T \alpha & \frac{1}{\alpha} \sin T \alpha \\
-\alpha \sin T \alpha & \cos T \alpha
\end{array}\right]\left[\begin{array}{l}
x \\
y
\end{array}\right]= \\
=\left[\begin{array}{cc}
-\alpha \sin T \alpha & \cos T \alpha \\
-\alpha^{2} \cos T \alpha & -\alpha \sin T \alpha
\end{array}\right]\left[\begin{array}{l}
x \\
y
\end{array}\right]=\left[\begin{array}{c}
-\alpha x \sin T \alpha+y \cos T \alpha \\
-\alpha^{2} x \cos T \alpha-\alpha y \sin T \alpha
\end{array}\right] .
\end{gathered}
$$

Logo, o polinômio $P=P(x, y)$ é dado por:

$$
P(x, y)=\left(t_{20} x^{2}+t_{02} y^{2}\right)\left[\begin{array}{c}
-\alpha x \sin T \alpha+y \cos T \alpha \\
-\alpha^{2} x \cos T \alpha-\alpha y \sin T \alpha
\end{array}\right] .
$$

Segue que o polinômio $P=P(x, y)$ pode ser escrito da forma:

$$
P(x, y)=\left(P_{1}(x, y), P_{2}(x, y)\right)
$$

Onde:

$$
\left\{\begin{array}{l}
P_{1}(x, y)=\left(-\alpha t_{20} \sin T \alpha\right) x^{3}+\left(t_{20} \cos T \alpha\right) x^{2} y+\left(-\alpha t_{02} \sin T \alpha\right) x y^{2}+\left(t_{02} \cos T \alpha\right) y^{3} . \\
P_{2}(x, y)=\left(-\alpha^{2} t_{20} \cos T \alpha\right) x^{3}+\left(-\alpha t_{20} \sin T \alpha\right) x^{2} y+\left(-\alpha^{2} t_{02} \cos T \alpha\right) x y^{2}+\left(-\alpha t_{20} \sin T \alpha\right) y^{3}
\end{array}\right.
$$

Por fim, para termos em mãos elementos suficientes para a análise do primeiro invariante de Birkhoff para as aplicações de Poincaré, temos o seguinte resultado auxiliar e o próximo corolário. 
Lema 5.2.2 As derivadas de ordem 2 da aplicação $\phi_{T}$ são todas nulas em $(x, y)=(0,0)$.

Demonstração: Segue do procedimento usual para o cálculo das derivadas do aplicação fluxo, relacionado a uma equação diferencial ordinária, conforme discutido em [33].

Corolário 5.2.2 O primeiro invariante de Birkhoff correspondente a aplicação de Poincaré $\psi_{0}$ é dado por:

$$
\tau=\frac{a_{10}}{8}\left(-a_{21}+3 \frac{b_{10}}{a_{10}} a_{03}-3 \frac{a_{01}}{b_{10}} b_{30}+b_{12}\right)-\frac{b_{10}}{8}\left(a_{12}-3 \frac{a_{01}}{b_{10}} a_{30}-\frac{a_{01}}{b_{10}} b_{21}+3 b_{03}\right),
$$

onde os coeficientes $a_{i, j}$ e $b_{i, j}$ são os coeficientes que aparecem na expressão de $J^{3} \psi_{0}(x, y)$.

Demonstração: Segue do fato de que $J^{3} \psi_{0}(x, y)=J^{3} \phi_{T}(x, y)+P(x, y)$ $e$ do fato de as derivadas de ordem 2 de $\psi_{0}$ serem as derivadas de ordem 2 de $\phi_{T}$, em $(0,0)$. Logo, todas são nulas, pelo lema anterior. Portanto, levando em consideração a fórmula que apresentamos, na seção anterior, para o cálculo do primeiro invariante, concluímos que o primeiro invariante para a aplicação $\psi_{0}$ é dado pela expressão do enunciado.

Vamos a análise do primeiro invariante para $\psi_{0}$.

\subsection{Análise do Primeiro Invariante de Birkhoff para $\psi_{0}$}

O primeiro invariante de Birkhoff correspondente a aplicação $\psi_{0}$ é dado pela corolário 5.2.2. Nessa expressão, podemos notar que o primeiro invariante depende linearmente das derivadas de terceira ordem de $\psi_{0}$ em $(x, y)=(0,0)$. Por outro lado, pela proposição 5.2, temos que as derivadas de terceira ordem de $\psi_{0}$, em $(0,0)$, são a soma da correspondente derivada da aplicação $\phi_{T}$ com "o elemento correspondente" do polinômio $P=P(x, y)$. Portanto, podemos pensar que o primeiro invariante de Birkhoff $\tau$, associado a aplicação $\psi_{0}$, é a soma de dois termos $\tau=\tau_{1}+\tau_{2}$, onde $\tau_{1}$ é obtido por meio das derivadas de terceira ordem de $\phi_{T}$ e $\tau_{2}$ é obtido por meio dos coeficientes do polinômio $P$.

Observação 5.3.1 O coeficiente $\tau_{1}$ é obtido por meio da igualdade dada no corolário 5.2.2, tomando-se os elementos correspondentes a derivadas de terceira ordem como nos elementos de $\phi_{T}$ e $\tau_{2}$ tomando-se os elementos de terceira ordem como nos elementos do polinômio P. Como a dependência do primeiro invariante em relação às derivadas de terceira ordem é linear, teremos $\tau=\tau_{1}+\tau_{2}$. 
Vamos, a seguir, calcular o termo $\tau_{2}$. Para este cálculo, teremos:

$$
\left[\begin{array}{ll}
a_{10} & a_{01} \\
b_{10} & b_{01}
\end{array}\right]=\left[\begin{array}{cc}
\cos T \alpha & \frac{1}{\alpha} \sin T \alpha \\
-\alpha \sin T \alpha & \cos T \alpha
\end{array}\right] .
$$

Segue então que:

- $\frac{b_{10}}{a_{01}}=\frac{-\alpha \sin T \alpha}{\frac{1}{\alpha} \sin T \alpha}=-\alpha^{2}$;

- $\frac{a_{01}}{b_{10}}=\frac{-1}{\alpha^{2}}$;

Os elementos correspondentes aos termos de terceira ordem estão na igualdade (5.1). Segue que:

- $\frac{a_{10}}{8}\left(-a_{21}+3 \frac{b_{10}}{a_{10}} a_{03}-3 \frac{a_{01}}{b_{10}} b_{30}+b_{12}\right)=\frac{\cos T \alpha}{8}\left\{-t_{20} \cos T \alpha-3 \alpha^{2} t_{02} \cos T \alpha-\right.$ $\left.3 \frac{1}{\alpha^{2}} \alpha^{2} \cos T \alpha-\alpha^{2} t_{02} \cos T \alpha\right\}=$

$$
=\frac{\cos ^{2} T \alpha}{8}\left\{-t_{20}-3 \alpha^{2} t_{02}-3 t_{20}-\alpha^{2} t_{02}\right\}=\frac{-\cos ^{2} T \alpha}{2}\left\{t_{20}+\alpha^{2} t_{02}\right\} .
$$

Por outro lado, teremos:

- $\frac{b_{10}}{8}\left(a_{12}-3 \frac{a_{01}}{b_{10}} a_{30}-\frac{a_{01}}{b_{10}} b_{21}+3 b_{03}\right)=$

$$
\begin{aligned}
& =\frac{-\alpha \sin T \alpha}{8}\left\{-\alpha t_{02} \sin T \alpha-\frac{3}{\alpha^{2}} \alpha t_{20} \sin T \alpha-\frac{1}{\alpha^{2}} \alpha t_{20} \sin T \alpha-3 \alpha t_{02} \sin T \alpha\right\} \\
& =\frac{-\alpha \sin ^{2} T \alpha}{8}\left\{-\alpha t_{02}-\frac{3}{\alpha} t_{20}-\frac{t_{20}}{\alpha}-3 \alpha t_{02}\right\} \\
& =-\frac{\sin ^{2} T \alpha}{8}\left\{-\alpha^{2} t_{02}-3 t_{20}-t_{20}-3 \alpha^{2} t_{02}\right\} \\
& =\frac{\sin ^{2} T \alpha}{2}\left\{\alpha^{2} t_{02}+t_{20}\right\}
\end{aligned}
$$

Segue então que:

$$
\begin{aligned}
& \tau_{2}=-\frac{\cos ^{2} T \alpha}{2}\left\{t_{20}+\alpha^{2} T_{02}\right\}-\frac{\sin ^{2} T \alpha}{2}\left\{\alpha^{2} t_{02}+t_{20}\right\} \\
& =\left(\cos ^{2} T \alpha+\sin ^{2} T \alpha\right) \frac{\left(-\alpha^{2} t_{02}-t_{20}\right)}{2} \\
& =-\frac{1}{2}\left(\alpha^{2} t_{02}+t_{20}\right)
\end{aligned}
$$

Utilizando a definição da função $e=e(x, y)$, de onde temos os coeficientes $t_{20}$ e $t_{02}$, obtemos: 


$$
t_{20}=-\frac{\alpha^{2} P_{2}^{\prime}(E)}{2} \text { e } t_{02}=-\frac{P_{2}^{\prime}(E)}{2}
$$

Portanto:

$$
\tau_{2}=-\frac{1}{2} \frac{\left(-\alpha^{2} P_{2}^{\prime}(E)-\alpha^{2} P_{2}^{\prime}(E)\right)}{2}=\frac{1}{2} \frac{2 \alpha^{2} P_{2}^{\prime}(E)}{2}=\frac{\alpha^{2} P_{2}^{\prime}(E)}{2} .
$$

Com isso, conseguimos uma expressão para o coeficiente $\tau_{2}$. Para o coeficiente $\tau$, obtemos o seguinte corolário.

Corolário 5.3.1 O primeiro invariante de Birkhoff, associado a aplicação $\psi_{0}$, é dado por:

$$
\tau=\tau_{1}+\frac{\alpha^{2} P_{2}^{\prime}(E)}{2}
$$

onde $\tau_{1}$ é calculado a partir das derivadas de terceira ordem da aplicação $\phi_{T}$, conforme mencionado na observação 5.3.1.

Demonstração: Segue dos cálculos feitos acima.

Notemos que o coeficiente $\tau_{1}$ depende das derivadas, até ordem 3 , de $\phi_{T}$ em $(0,0)$. Como o período $T=T(E)$ é uma função da energia, podemos considerar o coeficiente $\tau_{1}$ como função da energia e escrever $\tau_{1}=\tau_{1}(E)$. Devido a isso, obtemos o seguinte corolário.

Definição 5.3.1 Tendo em vista que o primeiro invariante de Birkhoff, da aplicação $\psi_{0}$, pode ser escrito como uma função da energia, definimos a seguinte função:

$$
I_{B}: J \longrightarrow \mathbb{R}
$$

Onde $I_{B}(E)$ é o primeiro invariante de Birkhoff da aplicação $\psi_{0}$.

Lembremos que a aplicação $P_{2}=P_{2}\left(E_{2}\right)$ associa a cada nível de energia $E_{2}>0$, do sistema Hamiltoniano $X_{H_{2}}$, o período da respectiva órbita periódica com energia $E_{2}$. Devemos notar que ao fazer a energia $E_{2}$ tender a 0 , a correspondente órbita de $X_{H_{2}}$ passa pelo eixo das ordenadas cada vez mais próximo da singularidade $(0,0)$ do campo $X_{H_{2}}$. Esta singularidade, por sua vez, é uma singularidade hiperbólica. Baseado nessa propriedade, é possível mostrar que:

Lema 5.3.1 Vale o seguinte limite, para a derivada da função $P_{2}$ :

$$
\lim _{E_{2} \rightarrow 0} P_{2}^{\prime}\left(E_{2}\right)=-\infty
$$


Demonstração: Nessas condições, o período, da órbita periódica, é inversamente proporcional a energia da órbita (algo como 1/ $E_{2}$ ).

Diante desse lema e da definição 5.3.1, teremos a próxima proposição que descreve o primeiro invariante de Birkhoff, para a aplicação $\psi_{0}$, quando a energia $E$ tende a zero

Proposição 5.3.1 Se o nível de energia $E$ da órbita periódica $\Gamma_{E}$ for suficientemente pequeno, então o primeiro invariante de Birkhoff, correspondente a aplicação $\psi_{0}$, será não nulo. Além disso, se o valor do parâmetro $\epsilon$ também for suficientemente pequeno, então o primeiro invariante de Birkhoff, correspondente às aplicações $\psi_{\epsilon}$, para $\epsilon$ pequeno, será não nulo.

Demonstração: Para a aplicação $\psi_{0}$, o primeiro invariante será não nulo, pelo lema anterior e corolário 5.3.1. Como o primeiro invariante depende das derivadas até terceira ordem, para $\epsilon$ suficientemente pequeno, as derivadas de terceira ordem de $\psi_{\epsilon}$, em $(0,0)$, estarão próximas das derivadas de $\psi_{0}$. Sendo o invariante não nulo para $\psi_{0}$, então vale o mesmo para $\psi_{\epsilon}$, quando $\epsilon$ for suficientemente pequeno.

Neste ponto, podemos demonstrar o Teorema 1.1.2.

Demonstração do Teorema 1.1.2: Seja $I_{B}: J \longrightarrow \mathbb{R}$ a função, que associa a cada nivel de energia $E$, o primeiro invariante de Birkhoff $I_{B}(E)$, correspondente a aplicação $\psi_{0}$ (definição 5.3.1), e suponhamos que $E \in J$ e que $I_{B}(E) \neq 0$. O primeiro invariante de Birkhoff, correspondente a $\psi_{\epsilon}$, depende das derivadas até terceira ordem $\psi_{\epsilon}$ em $(0,0)$ e estas variam continuamente com o parâmetro $\epsilon$. Logo, sendo $I_{B}(E) \neq 0$, existe $\epsilon_{0}=\epsilon_{0}(E)>0$, tal que se $|\epsilon|<\epsilon_{0}$, então o primeiro invariante de Birkhoff, correspondente a $\psi_{\epsilon}$, é não nulo. Segue do teorema 4.1.2, que existem curvas fechadas e invariantes, por $\psi_{\epsilon}$, que se acumulam em $(0,0)$, quando $|\epsilon|<\epsilon_{0}$. Estas curvas fechadas invariantes, analisada ao longo da órbita periódica $\Gamma_{E}$, de $X_{H_{\epsilon}}$, correspondem a toros invariantes se acumulando em $\Gamma_{E}$.

No próximo capítulo, com o objetivo de criar ferramentas para provar o Teorema 1.1.3, apresentaremos um critério perturbativo para a análise da persistência de curvas rotacionais invariantes para aplicações twist. 


\section{Capítulo 6}

\section{Destruição de Curvas Invariantes.}

Objetivo deste capítulo é apresentar um método (perturbativo, chamado Critério de Melnikov) para a destruição de curvas rotacionais invariantes para aplicações twist, para que, no próximo capítulo, com base neste critério, possamos dar uma condição de destruição de toros invariantes nas coordenadas da seção de Poincaré $\Sigma_{E}^{+}$. As principais referências utilizadas, neste capítulo, foram [28] e [29].

\subsection{Critério de Melnikov}

Seja $f: \mathbb{S}^{1} \times(a, b) \longrightarrow \mathbb{S}^{1} \times(a, b)$ um difeomorfismo satisfazendo a condição twist:

$$
\text { Se } f(\theta, r)=(\Theta, R) \text {, então } \frac{\partial \Theta}{\partial r}(\theta, r) \neq 0, \forall(\theta, r) \in \mathbb{S}^{1} \times(a, b) \text {. }
$$

Iremos fazer, a respeito de $f$, as seguintes hipóteses:

- $f$ satisfaz uma certa rigidez nos bordos de $\mathbb{S}^{1} \times(a, b)$. Indicando por $F: \mathbb{R} \times(a, b) \longrightarrow \mathbb{R} \times(a, b)$ um levantamento de $f$, então existem números reais $\omega_{-}<\omega_{+}$, satisfazendo:

$$
F(x, a)=\left(x+\omega_{-}, a\right) \text { e } F(x, b)=\left(x+\omega_{+}, b\right) .
$$

Assim, a aplicação $f$ pode ser estendida aos bordos de $\mathbb{S}^{1} \times(a, b)$, de modo que seja uma rotação rígida.

- Fazendo $\lambda=r d \theta$, existe $s: \mathbb{S}^{1} \times(a, b) \longrightarrow \mathbb{R}$, tal que: 


$$
d s=f^{*} \lambda-\lambda .
$$

Nesse caso, se $F: \mathbb{R} \times(a, b) \rightarrow \mathbb{R} \times(a, b)$ é um levantamento de $f$, então podemos associar um princípio variacional às órbitas de $F$. Se $F(x, y)=(X, Y)$, então existe uma função $S: \mathbb{R}^{2} \rightarrow \mathbb{R}$, tal que $d S=$ $Y d X-y d x$. Por outro lado, em consequência da condição twist, $B$ : $\mathbb{R}^{2} \rightarrow \mathbb{R}^{2}$, definida por $B(x, y)=(x, X(x, y))$ é um difeomorfismo sobre sua imagem (aplicando-se o Teorema da Função Inversa). Definimos $h(x, X)=S\left(B^{-1}(x, X)\right)$. Dessa forma, teremos:

$$
F(x, y)=(X, Y) \Longleftrightarrow y=-\frac{\partial h}{\partial x}(x, X) \text { e } Y=\frac{\partial h}{\partial X}(x, X)
$$

Dessas relações, obtemos o princípio variacional, conforme discutido na parte introdutória desse texto.

Observação 6.1.1 A função $h=h(x, X)$ será chamada de princípio variacional. Temos as funções geradoras $s$ e $S$ (no anel e no recobrimento do anel, respectivamente). As relações entre essas funções (função geradora para o simplectomorfismo e princípio variacional) estão muito bem descritas em [14], por exemplo.

Diante dessas condições, $f$ é uma aplicação twist simplética exata no anel $\mathbb{S}^{1} \times(a, b)$, cujas órbitas satisfazem a um princípio variacional. Vamos supor que tenhamos uma perturbação dessa aplicação $f$ por aplicações twist simpléticas exatas, em $\mathbb{S}^{1} \times(a, b)$, da forma $f_{\epsilon}=f+o(\epsilon)$. Iremos supor, também, que exista $\Gamma \subseteq \mathbb{S}^{1} \times(a, b)$, curva rotacional invariante (que indicaremos, abreviadamente, por CRI) para $f$, que seja $(m, n)$-ressonante. Assim, toda órbita de $f$ sobre o subconjunto invariante $\Gamma$ é $n$-periódica e possui número de rotação $\frac{m}{n}$. Gostaríamos de avaliar se esta curva rotacional invariante persiste ou não sob a perturbação $f_{\epsilon}=f+o(\epsilon)$. Apresentaremos um método para analisar esta questão, baseado em [28]. Daremos, a seguir, uma definição formal dessa noção de persistência.

Definição 6.1.1 Dizemos que a curva $\Gamma \subseteq \mathbb{S}^{1} \times(a, b)$, curva rotacional invariante para $f$, persiste sob a perturbação $f_{\epsilon}=f+o(\epsilon)$, se $f_{\epsilon}$ possui, para todo $\epsilon$ suficientemente pequeno, uma curva rotacional invariante $\Gamma_{\epsilon} \subseteq$ $\mathbb{S}^{1} \times(a, b)$, que (também) é $(m, n)$-ressonante e satisfaça:

$$
\Gamma_{\epsilon}=\Gamma+o(\epsilon)
$$


Sendo $\Gamma$ uma curva rotacional invariante para $f$, segue, do teorema de Birkhoff, que existe uma função $v: \mathbb{S}^{1} \longrightarrow(a, b)$, tal que $\Gamma=\operatorname{graf}(v)$. Além disso, este teorema nos diz que essa função é, no mínimo, Lipschitz; no entanto, iremos supor aqui que $v$ seja suave. Naturalmente, temos o levantamento de $v, \tilde{v}: \mathbb{R} \longrightarrow(a, b)$, cujo gráfico é invariante por $F$, levantamento de $f$. Ao fazermos a perturbação $f_{\epsilon}=f+o(\epsilon)$, num certo sentido falando, a curva $\Gamma$ dará origem a duas outras novas curvas, que indicaremos por $\Gamma_{\epsilon}$ e $\Gamma_{\epsilon}^{*}$. A curva $\Gamma_{\epsilon}$ representaria a curva $\Gamma$, dentro do sistema perturbado, e $\Gamma_{\epsilon}^{*}$ representaria $f_{\epsilon}^{n}\left(\Gamma_{\epsilon}\right)$. Iremos formalizar esses comentários no próximo lema. Seguirá que obter persistência da curva rotacional $\Gamma$ significará a igualdade $\Gamma_{\epsilon}=\Gamma_{\epsilon}^{*}$ (no entanto, não há razão, a priori, para valer essa igualdade). Vamos ao lema.

Lema 6.1.1 Supondo que estejamos nas condições descritas acima, se $|\epsilon|$ for suficientemente pequeno, então existem funções:

$$
v_{\epsilon}, v_{\epsilon}^{*}: \mathbb{S}^{1} \longrightarrow(a, b),
$$

satisfazendo as seguintes condições:

1. $v_{\epsilon}(x)=v(x)+o(\epsilon)$ e $v_{\epsilon}^{*}(x)=v(x)+o(\epsilon), \forall x \in \mathbb{S}^{1}$.

2. $f_{\epsilon}^{n}\left(x, v_{\epsilon}(x)\right)=\left(x, v_{\epsilon}^{*}(x)\right)$.

Indicamos $\Gamma_{\epsilon}=\operatorname{graf}\left(v_{\epsilon}\right)$ e $\Gamma_{\epsilon}^{*}=\operatorname{graf}\left(v_{\epsilon}^{*}\right)$.

Demonstração: $\operatorname{Ver}$ [28].

Analisando-se a construção da prova desse lema em [28], é imediato o seguinte corolário.

Corolário 6.1.1 Nas condições acima, a curva $\Gamma$ persiste sob a perturbação $f_{\epsilon}=f+o(\epsilon)$ se, e somente se, $\Gamma_{\epsilon}=\Gamma_{\epsilon}^{*}$. Ou seja, as funções $v_{\epsilon}$ e $v_{\epsilon}^{*}$ coincidem.

Portanto, para a análise da persistência da curva $\Gamma$, é útil quantificar a separação entre os gráficos das funções $v_{\epsilon} \mathrm{e} v_{\epsilon}^{*}$. Iremos apresentar um critério, nesse sentido, que será resumido no próximo corolário.

Consideremos os levantamentos, dos gráficos, $\tilde{v}_{\epsilon}, \tilde{v}_{\epsilon}^{*}: \mathbb{R} \longrightarrow(a, b)$. Podemos definir uma função $\tilde{L}_{\epsilon}: \mathbb{R} \longrightarrow \mathbb{R}$ tal que:

$$
\tilde{L}_{\epsilon}^{\prime}(x)=\tilde{v}_{\epsilon}^{*}(x)-\tilde{v}_{\epsilon}(x), \forall x \in \mathbb{R} .
$$

Assim, temos uma função $L_{\epsilon}: \mathbb{S}^{1} \longrightarrow \mathbb{R}$, tal que $L_{\epsilon}^{\prime}=v_{\epsilon}^{*}-v_{\epsilon}$.

Analisando-se a expansão de $L_{\epsilon}$, com respeito ao parâmetro $\epsilon$, temos:

$$
L_{\epsilon}=L_{0}+\epsilon L_{1}+o\left(\epsilon^{2}\right) .
$$


Logo, como $L_{0}^{\prime}(x)=v_{0}^{*}(x)-v_{0}(x)=0, \forall x \in \mathbb{S}^{1}$, ou seja, a função $L_{0}$ é constante, assim:

$$
L_{\epsilon}^{\prime}(x)=\epsilon L_{1}^{\prime}(x)+o\left(\epsilon^{2}\right)=v_{\epsilon}^{*}(x)-v_{\epsilon}(x) .
$$

Segue então que se existe $x_{0} \in \mathbb{S}^{1}$ tal que $L_{1}^{\prime}\left(x_{0}\right) \neq 0$, então, para $\epsilon$ suficientemente pequeno, podemos garantir que $v_{\epsilon}^{*}\left(x_{0}\right)-v_{\epsilon}\left(x_{0}\right) \neq 0$. Ou seja, $\Gamma_{\epsilon} \neq \Gamma_{\epsilon}^{*}$ e, com isso, a curva rotacional invariante $\Gamma$ não persiste com a perturbação $f_{\epsilon}=f+o(\epsilon)$. Essa argumentação está resumida no próximo corolário.

Corolário 6.1.2 Se a função $L_{1}: \mathbb{S}^{1} \longrightarrow \mathbb{R}$ não é uma função constante, então a curva $\Gamma$ não persiste sob a perturbação $f_{\epsilon}=f+o(\epsilon)$.

A questão imediata que surge é como expressar essa tal função $L_{1}$. O fato é que ela se relaciona com as funções $h_{\epsilon}$, associadas a cada função $f_{\epsilon}$. Com o intuito de expressar esta relação, consideremos, a seguir, a expansão em série de Taylor de $h_{\epsilon}$, com respeito ao parâmetro $\epsilon$. Temos:

$$
h_{\epsilon}=h_{0}+\epsilon h_{1}+o\left(\epsilon^{2}\right) .
$$

O termo $L_{1}$ se relaciona com o termo $h_{1}$, da expansão de $h_{\epsilon}$. Este é o conteúdo da próxima proposição.

Proposição 6.1.1 A função $\tilde{L}_{\epsilon}: \mathbb{R} \rightarrow \mathbb{R}$ é dada por:

$$
\tilde{L}_{\epsilon}(x)=\sum_{j=0}^{n-1} h_{\epsilon}\left(\bar{x}_{j}(x, \epsilon), \bar{x}_{j+1}(x, \epsilon), \text { onde } \bar{x}_{j}(x, \epsilon)=\pi_{1}\left(F_{0}^{j}(x, \tilde{v}(x))\right) .\right.
$$

Estamos indicando $\pi_{1}: \mathbb{R}^{2} \rightarrow \mathbb{R}$ a projeção na primeira coordenada.

Demonstração: $\operatorname{Ver}$ [28].

Diante dessa expressão relacionando $\tilde{L}_{\epsilon}$ e o princípio variacional $h_{\epsilon}$, obtemos:

Corolário 6.1.3 Vale a igualdade:

$$
\tilde{L}_{1}(x)=\sum_{j=0}^{n-1} h_{1}\left(x_{j}, x_{j+1}\right), \text { onde } x_{j}=\pi\left(F^{j}(x, \tilde{v}(x))\right) .
$$

Demonstração: $\operatorname{Ver}$ [28]. 


\subsection{Critério de Melnikov Atraves da Função Geradora.}

Como foi ressaltado no início da seção anterior, o princípio variacional $h$ está relacionado à função geradora $S$, via a mudança de coordenadas induzida pela condição twist. Na seção anterior a função $L_{1}$ foi escrita em termos do princípio variacional mas para os propósitos deste texto, é mais conveniente escrever a função $L_{1}$ em termos das funções geradoras no anel. Isso é feito na próxima proposição, cuja demonstração está em [29] e iremos reproduzi-la aqui.

Proposição 6.2.1 Seja $\tilde{L}_{1}: \mathbb{R} \rightarrow \mathbb{R}$ a função correspondente ao potencial de Melnikov, como indicado acima. Então vale a igualdade ${ }^{1}$ :

$$
\tilde{L}_{1}(x)=\sum_{j=0}^{n-1}\left\{S_{1}\left(x_{j}, y_{j}\right)-\tilde{\lambda}_{F_{0}\left(x_{j}, y_{j}\right)}\left(F_{1}\left(x_{j}, y_{j}\right)\right)\right\},
$$

onde $\left(x_{j}, y_{j}\right)=F^{j}(x, \tilde{v}(x))$ e $\tilde{\lambda}=y d x$.

Demonstração: Observemos que se $F_{\epsilon}(x, y)=\left(X_{\epsilon}, Y_{\epsilon}\right)$, então:

$$
h_{\epsilon}\left(x, X_{\epsilon}(x, y)\right)=S_{\epsilon}(x, y) .
$$

Derivando esta igualdade com respeito a $\epsilon$ e calculando em $\epsilon=0$, obtemos:

$$
h_{1}\left(x, X_{0}(x, y)\right)+\frac{\partial h_{0}}{\partial X}\left(x, X_{0}(x, y)\right) X_{1}(x, y)=S_{1}(x, y) .
$$

Mas observando que $\frac{\partial h_{0}}{\partial X}\left(x, X_{0}(x, y)\right)=Y_{0}(x, y)$, podemos escrever:

$$
h_{1}\left(x, X_{0}(x, y)\right)=S_{1}(x, y)-Y_{0}(x, y) X_{1}(x, y) .
$$

Analisando o último termo desta igualdade, podemos notar que:

$$
\tilde{\lambda}_{F_{0}(x, y)}\left(F_{1}(x, y)\right)=\tilde{\lambda}_{\left(X_{0}, Y_{0}\right)}\left(X_{1}, Y_{1}\right)=Y_{0}(x, y) X_{1}(x, y) .
$$

Diante dessa igualdade, segue que:

$$
\tilde{L}_{1}(x)=\sum_{j=0}^{n-1}\left\{S_{1}\left(x_{j}, y_{j}\right)-\tilde{\lambda}_{F_{0}\left(x_{j}, y_{j}\right)}\left(F_{1}\left(x_{j}, y_{j}\right)\right)\right\} .
$$

Com isso, concluímos a demonstração.

\footnotetext{
${ }^{1}$ Ver a próxima observação, refrente ao termo $\tilde{\lambda}_{F_{0}(x, y)}\left(F_{1}(x, y)\right)$.
} 
Observação 6.2.1 Observemos que o termo $\tilde{\lambda}_{F_{0}\left(x_{j}, y_{j}\right)}\left(F_{1}\left(x_{j}, y_{j}\right)\right)$, que aparece na proposição anterior, não corresponde a um pullback da 1-forma $\tilde{\lambda}$. Além disso, é conveniente analisar o termo relacionado a $F_{1}$ como um campo de vetores.

Com isso, conseguimos expressar $\tilde{L}_{1}$ em termos da função $S_{1}$. Para a aplicação que faremos, seria ainda mais interessante ter a igualdade da proposição anterior em $\mathbb{S}^{1} \times(a, b)$. Faremos isso baseado nas relações entre as funções $S$ e $s$, que estão descritas em [14].

Proposição 6.2.2 A projeção $L_{1}: \mathbb{S}^{1} \rightarrow \mathbb{R}$, da função $\tilde{L}_{1}$ da proposição anterior, é dada por:

$$
L_{1}(\theta)=\sum_{j=0}^{n-1}\left\{s_{1}\left(\theta_{j}, r_{j}\right)-\lambda_{f_{0}\left(\theta_{j}, r_{j}\right)}\left(f_{1}\left(\theta_{j}, r_{j}\right)\right)\right\}
$$

Onde $\left(\theta_{j}, r_{j}\right)=\varphi^{j}(\theta, v(\theta)), 0 \leq j \leq n-1$.

Demonstração: Seja $\left(x_{j}, y_{j}\right)_{j}$ o levantamento da órbita de $(\theta, v(\theta))$. Se $p: \mathbb{R} \times(a, b) \rightarrow \mathbb{S}^{1} \times(a, b)$ é o recobrimento, façamos $p\left(x_{j}, y_{j}\right)=\left(\theta_{j}, r_{j}\right)$. Assim, segue que:

$$
S_{1}\left(x_{j}, y_{j}\right)=s_{1}\left(p\left(x_{j}, y_{j}\right)\right)=s_{1}\left(\theta_{j}, r_{j}\right) .
$$

Por outro lado, observemos que:

$$
\begin{aligned}
& \lambda_{f_{0}\left(\theta_{j}, r_{j}\right)}\left(f_{1}\left(\theta_{j}, r_{j}\right)\right)=\lambda_{f_{0}\left(p\left(x_{j}, y_{j}\right)\right)}\left(f_{1}\left(p\left(x_{j}, y_{j}\right)\right)\right)=\lambda_{p\left(F_{0}\left(x_{j}, y_{j}\right)\right)}\left(\left.\frac{\partial}{\partial \epsilon}\right|_{\epsilon=0} f_{\epsilon}\left(p\left(x_{j}, y_{j}\right)\right)\right)= \\
& =\lambda_{p\left(F_{0}\left(x_{j}, y_{j}\right)\right)}\left(\left.\frac{\partial}{\partial \epsilon}\right|_{\epsilon=0} p\left(F_{\epsilon}\left(x_{j}, y_{j}\right)\right)\right)=\lambda_{p\left(F_{0}\left(x_{j}, y_{j}\right)\right)}\left(d_{F_{0}\left(x_{j}, y_{j}\right)} p\left(F_{1}\left(x_{j}, y_{j}\right)\right)=\right. \\
& =\left(p^{*} \lambda\right)_{F_{0}\left(x_{j}, y_{j}\right)}\left(F_{1}\left(x_{j}, y_{j}\right)\right) .
\end{aligned}
$$

Logo, utilizando a proposição anterior:

$$
\tilde{L}_{1}(x)=\sum_{j=0}^{n-1}\left\{s_{1}\left(\theta_{j}, r_{j}\right)-\lambda_{f_{0}\left(\theta_{j}, r_{j}\right)}\left(f_{1}\left(\theta_{j}, r_{j}\right)\right)\right\}=L_{1}(\theta)=L_{1}(p(x)) .
$$

Indicando por $p$ o recobrimento de $\mathbb{S}^{1}$. Segue o resultado. 


\section{Capítulo 7}

\section{A Função de Melnikov na Seção}

Objetivo deste capítulo é expressar a condição de Melnikov, sobre destruição de curvas rotacionais invariantes, nas coordenadas da seção $\Sigma_{E}^{+}$, tendo em vista que as aplicações de Poincaré são conjugadas a aplicações twist, conforme discutido no Capítulo 4. Por meio disso, estabeleceremos uma condição de destruição de toros invariantes em termos de elementos do campo $X_{H_{\epsilon}}$.

\subsection{Algumas considerações iniciais.}

Como as funções que irão aparecer, na sequência do texto, dependem do parâmetro $\epsilon$ e teremos que derivar, em muitas situações, em relação ao parâmetro $\epsilon$ e calcular em $\epsilon=0$, fixaremos, a princípio, a seguinte notação. Se $\xi=\xi_{\epsilon}(z)$ é uma função diferenciável que depende de um parâmetro $\epsilon$, então indicaremos por $\xi_{1} \doteq \xi_{1}(z)=\left.\frac{\partial}{\partial \epsilon}\right|_{\epsilon=0} \xi_{\epsilon}(z)$.

Fixemos $E \in J$ e suponhamos que $I_{B}(E) \neq 0$. Nesse caso, existe $\epsilon_{0}=\epsilon_{0}(E)>0$, tal que se $|\epsilon|<\epsilon_{0}$, então $\Gamma_{E}$ é uma órbita periódica, de $X_{H_{\epsilon}}$, elíptica em seu nível de energia. Além disso, indicando por $\psi_{\epsilon}: \Omega_{\epsilon} \rightarrow \Omega_{\epsilon}$ a correspondente Transformação de Poincaré, nas coordenadas da seção $\Sigma_{E}^{+}$, podemos fazer uso da Forma Normal de Birkhoff, para o estudo dessas aplicações em coordenadas polares. Segue, pelo Teorema 4.1.2, que existem curvas invariantes por $\psi_{\epsilon}$, em $\Omega_{\epsilon} \backslash\{(0,0)\}$, que se acumulam em $(0,0)$. Logo, podemos delimitar, para cada $\psi_{\epsilon}$, regiões anulares invariantes, arbitrariamente próximas a $(0,0)$ (regiões entre curvas invariantes).

Indicando por $\bar{\Omega}_{\epsilon} \subseteq \Omega_{\epsilon} \backslash\{(0,0)\}$ uma região anular invariante por $\psi_{\epsilon}$, pelo resultado do Capítulo 4 , temos a mudança de coordenadas $G: \bar{\Omega}_{\epsilon} \rightarrow \overline{\mathbb{A}}_{\epsilon}$, que transforma $\psi_{\epsilon}: \bar{\Omega}_{\epsilon} \rightarrow \bar{\Omega}_{\epsilon}$ em uma transformação twist simplética exata $\varphi_{\epsilon}: \overline{\mathbb{A}}_{\epsilon} \rightarrow \overline{\mathbb{A}}_{\epsilon}$, sendo $\overline{\mathbb{A}}_{\epsilon}$ uma região anular invariante em $\mathbb{S}^{1} \times(0,+\infty)$, que depende do parâmetro $\epsilon$. Cada curva invariante por $\psi_{\epsilon}$, em $\Omega_{\epsilon} \backslash\{(0,0)\}$ 
corresponde a uma curva rotacional invariante por $\varphi_{\epsilon}$. Portanto, temos, associado a ela, um número de rotação. Para o caso $\epsilon=0$, o domínio $\Omega_{0} \backslash\{(0,0)\}$ é folheado por curvas invariantes e, portanto, $\varphi_{0}$ é uma aplicação twist integrável.

Fixemos $\Gamma \subseteq \Omega_{0} \backslash\{(0,0)\}$ uma curva invariante por $\psi_{0}$, com número de rotação racional $\frac{m}{n}$. A imagem de $\Gamma$, por $G, \tilde{\Gamma}=G(\Gamma) \subseteq \mathbb{A}_{0}$, é uma curva rotacional invariante por $\varphi_{0}: \mathbb{A}_{0} \rightarrow \mathbb{A}_{0}$, que é $(m, n)$-ressonante. Como há uma ordenação de curvas invariantes, segundo o seu número de rotação, podemos delimitar uma região anular invariante $\bar{\Omega}_{0} \subseteq \Omega_{0} \backslash\{(0,0)\}$, cujos bordos sejam curvas invariantes com número de rotação diofantino (curvas do tipo KAM, ver [14]) e tal que $\Gamma$ esteja contida no interior de $\bar{\Omega}_{0}$. Para $\epsilon \neq 0$, suficientemente pequeno, as curvas invariantes que formam o bordo de $\bar{\Omega}_{0}$ persistem, dando origem a uma região anular invariante por $\psi_{\epsilon}, \bar{\Omega}_{\epsilon} \subseteq$ $\Omega_{\epsilon} \backslash\{(0,0)\}$, cujos bordos são curvas invariantes com os mesmos números de rotação das curvas invariantes por $\psi_{0}$, que formam o bordo de $\bar{\Omega}_{0}$ e tal que a curva $\Gamma$ está contida no interior de $\bar{\Omega}_{\epsilon}$.

Gostaríamos de avaliar a persistência dessa curva invariante $\Gamma$ (ou da curva $\tilde{\Gamma}=G(\Gamma)$, em coordenadas twist). Ao projetarmos a situação descrita no parágrafo anterior em $\mathbb{S}^{1} \times(0,+\infty)$, via a mudança de coordenadas $G$, não temos, exatamente, a situação apresentada na exposição do critério de Melnikov, no capítulo anterior, pois, nessa nova situação, o domínio da aplicação twist varia com o parâmetro $\epsilon$. No entanto, o critério de Melnikov ainda é válido, conforme estabelecido na próxima proposição.

Proposição 7.1.1 Consideremos a situação que vem sendo descrita nessa seção. Temos a familia de aplicações twist simplética exata $\varphi_{\epsilon}: \overline{\mathbb{A}}_{\epsilon} \rightarrow \overline{\mathbb{A}}_{\epsilon}$, com as respectivas funções geradoras $s_{\epsilon}: \overline{\mathbb{A}}_{\epsilon} \rightarrow \mathbb{R}$, sendo $G\left(\bar{\Omega}_{\epsilon}\right)=\overline{\mathbb{A}}_{\epsilon}$ e $\tilde{\Gamma}=$ $G(\Gamma)$ a curva $(m, n)$-ressonante, para $\varphi_{0}$ fixada, que é gráfico de $v: \mathbb{S}^{1} \rightarrow \mathbb{R}$. Nessa situação, consideremos a função $L_{1}: \mathbb{S}^{1} \rightarrow \mathbb{R}$, definida por:

$$
\bar{L}_{1}(\theta)=\sum_{j=0}^{n-1}\left\{s_{1}\left(\theta_{j}, r_{j}\right)-\lambda_{\varphi_{0}\left(\theta_{j}, r_{j}\right)}\left(\varphi_{1}\left(\theta_{j}, r_{j}\right)\right)\right\},
$$

onde $\left(\theta_{j}, r_{j}\right)=\varphi_{0}^{j}(\theta, v(\theta))$. Se a função $\bar{L}_{1}$ não é uma função constante, então a curva $\tilde{\Gamma}$ não persiste sob a pertubação $\varphi_{\epsilon}=\varphi_{0}+o(\epsilon)$.

Demonstração: A demonstração dessa proposição pode ser feita através dos mesmos passos que foram feitos para provar o critério de Melnikov, feito no capitulo anterior, observando que a demonstração depende apenas da perturbação $f_{\epsilon}=f_{0}+o(\epsilon)$ sobre a curva $(m, n)$-ressonante, em que a análise é feita. É importante observar que, na situação deste capítulo, temos a curva $(m, n)$-ressonante contida no interior dos anéis compactos $\overline{\mathbb{A}}_{\epsilon}$. Devido a esse 
fato, todos os passos da demonstração do critério de Melnikov, feitos no capitulo anterior, podem ser adaptados.

Observação 7.1.1 Ao longo deste texto, iremos supor que a curva $(m, n)$ ressonante $\Gamma$ é fixada suficientemente próxima do ponto fixo, tendo em vista que a construção da região anular invariante pode ser feita, arbitrariamente, próxima ao ponto fixo, partindo de uma curva ressonante que esteja suficientemente próxima ao ponto fixo.

Portanto, a persistência da curva rotacional $\tilde{\Gamma}$ (ou, equivalentemente, da curva invariante $\Gamma \subseteq \Omega_{0} \backslash\{(0,0)\}$ por $\left.\psi_{0}\right)$, sob a perturbação $\varphi_{\epsilon}=\varphi_{0}+o(\epsilon)$ pode ser analisada por meio da função $\bar{L}_{1}: \mathbb{S}^{1} \rightarrow \mathbb{R}$, dada por:

$$
\bar{L}_{1}(\theta)=\sum_{j=0}^{n-1}\left\{s_{1}\left(\theta_{j}, r_{j}\right)-\lambda_{f_{0}\left(\theta_{j}, r_{j}\right)}\left(f_{1}\left(\theta_{j}, r_{j}\right)\right)\right\}
$$

onde $\left(\theta_{j}, r_{j}\right)=\varphi_{0}^{j}(\theta, v(\theta)), 0 \leq j \leq n-1$.

Vamos analisar, separadamente, os dois termos do somatório que compõe a expressão de $\bar{L}_{1}$. Sendo assim, definiremos: $\bar{M}_{1}: \mathbb{S}^{1} \rightarrow \mathbb{R}$ e $\bar{N}_{1}: \mathbb{S}^{1} \rightarrow \mathbb{R}$, dados por:

$$
\bar{M}_{1}(\theta)=\sum_{j=0}^{n-1} s_{1}\left(\theta_{j}, r_{j}\right) \text { e } \bar{N}_{1}(\theta)=\sum_{j=0}^{n-1} \lambda_{f_{0}\left(\theta_{j}, r_{j}\right)}\left(f_{1}\left(\theta_{j}, r_{j}\right)\right)
$$

Dessa forma: $\bar{L}_{1}(\theta)=\bar{M}_{1}(\theta)-\bar{N}_{1}(\theta)$. Iremos trazer as expressões de $\bar{M}_{1}$ e $\bar{N}_{1}$ para as coordenadas em $\Omega$.

\subsection{Análise do termo $\bar{M}_{1}$.}

Vamos indicar $\bar{M}_{\epsilon}(\theta)=\sum_{j=0}^{n-1} s_{\epsilon}\left(\theta_{j}, r_{j}\right)$. Fixemos $z \in \Omega_{0}$ e $\theta_{z} \in \mathbb{S}^{1}$, tal que $G(z)=\left(\theta_{z}, v\left(\theta_{z}\right)\right)$

Lema 7.2.1 Vale a igualdade:

$$
\bar{M}_{\epsilon}\left(\theta_{z}\right)=\sum_{j}\left(s_{\epsilon} \circ G \circ \psi_{0}^{-1}\right)\left(\psi_{0}^{j}(z)\right) .
$$

Demonstração: Pela definição de $\bar{M}_{\epsilon}$ : 


$$
\bar{M}_{\epsilon}\left(\theta_{z}\right)=\sum_{j} s_{\epsilon}\left(\varphi_{0}^{j}\left(\theta_{z}, v\left(\theta_{z}\right)\right)\right)=\sum_{j=0}^{n-1} s_{\epsilon}\left(\varphi_{0}^{j}(G(z))\right) .
$$

Como $G$ é uma conjugação entre $\psi_{\epsilon}$ e $\varphi_{\epsilon}$, teremos:

$$
\bar{M}_{\epsilon}\left(\theta_{z}\right)=\sum_{j} s_{\epsilon}\left(G\left(\psi_{0}^{j}(z)\right)\right) .
$$

Mas sendo esta soma, uma soma sobre uma órbita periódica, conseguimos:

$$
\bar{M}_{\epsilon}\left(\theta_{z}\right)=\sum_{j}\left(s_{\epsilon} \circ G \circ \psi_{0}^{-1}\right)\left(\psi_{0}^{j}(z)\right) .
$$

Segue o resultado.

Dessa forma, relacionamos $\bar{M}_{\epsilon}$, de uma soma em órbitas de $\varphi_{0}$ a uma soma sobre órbitas de $\psi_{0}$.

Seja $f_{\epsilon}: \Omega \rightarrow \Omega$, definida por $f_{\epsilon}=\psi_{\epsilon} \circ \psi_{0}^{-1}$. Observemos que $f_{\epsilon}$ é um simplectomorfismo, com $f_{\epsilon}(0,0)=(0,0), \forall \epsilon$ e $\psi_{\epsilon}=f_{\epsilon} \circ \psi_{0}$. A seguir, provaremos duas igualdades que nos serão auxiliares.

Lema 7.2.2 Valem $^{1}$ as seguintes igualdades:

- $d\left(s_{\epsilon} \circ G\right)=\psi_{\epsilon}^{*} \mu-\mu$;

- $d\left(s_{\epsilon} \circ G \circ \psi_{0}^{-1}\right)=f_{\epsilon}^{*} \mu-\left(\psi_{0}^{-1}\right)^{*} \mu$;

Demonstração: Segue que:

$$
d\left(s_{\epsilon} \circ G\right)=G^{*} d s_{\epsilon}=G^{*}\left(\varphi_{\epsilon}^{*} \lambda-\lambda\right)=\psi_{\epsilon}^{*} G^{*} \lambda-G^{*} \lambda=\psi_{\epsilon}^{*} \mu-\mu .
$$

Por outro lado, temos:

$$
d\left(s_{\epsilon} \circ G \circ \psi_{0}^{-1}\right)=\left(\psi_{0}^{-1}\right)^{*} d\left(s_{\epsilon} \circ G\right)=\left(\psi_{0}^{-1}\right)^{*}\left(\psi_{0}^{*} f_{\epsilon}^{*} \mu-\mu\right)=f_{\epsilon}^{*} \mu-\left(\psi_{0}^{*}\right) \mu .
$$

Seguem as duas igualdades do enunciado.

Essas igualdades, juntamente com o teorema fundamental do cálculo, nos permite escrever:

Proposição 7.2.1 Vale a igualdade:

$$
\bar{M}_{\epsilon}\left(\theta_{z}\right)=\sum_{j=0}^{n-1} \int_{\gamma_{j}} d\left(s_{\epsilon} \circ G \circ \psi_{0}^{-1}\right)+n\left(s_{\epsilon} \circ G \circ \psi_{0}^{-1}\right)(z),
$$

\footnotetext{
${ }^{1} \mathrm{~A} 1$-forma $\mu$ foi definida na seção 4 , assim como a 1-forma $\lambda$.
} 
onde, $\gamma_{j}:\left[0, T_{j}\right] \longrightarrow \bar{\Omega}_{0}$, tal que $\gamma_{j}(0)=z$ e $\gamma_{j}\left(T_{j}\right)=\psi_{0}^{j}(z)$.

Demonstração: Observemos que:

$$
\begin{aligned}
& \bar{M}_{\epsilon}\left(\theta_{z}\right)=\sum_{j}\left(s_{\epsilon} \circ G \circ \psi_{0}^{-1}\right)\left(\psi_{0}^{j}(z)\right)= \\
& \quad=\sum_{j}\left\{\left(s_{\epsilon} \circ G \circ \psi_{0}^{-1}\right)\left(\psi_{0}^{j}(z)\right)-\left(s_{\epsilon} \circ G \circ \psi_{0}^{-1}(z)\right)\right\}+n s_{\epsilon} \circ G \circ \psi_{0}^{-1}(z) \\
& \quad=\sum_{j} \int_{\gamma_{j}} d\left(s_{\epsilon} \circ G \circ \psi_{0}^{-1}\right)+n\left(s_{\epsilon} \circ G \circ \psi_{0}^{-1}\right)(z) .
\end{aligned}
$$

Obtemos, assim, a igualdade do enunciado.

Corolário 7.2.1 Segue, da proposição anterior, que:

$$
\bar{M}_{\epsilon}(z)=\int_{\gamma_{j}}\left(f_{\epsilon}^{*} \mu-\left(\psi_{0}^{-1}\right)^{*} \mu\right)+n s_{\epsilon} \circ G \circ \psi_{0}^{-1}(z) .
$$

Demonstração: Segue dos últimos lemas e da proposição anterior.

Observação 7.2.1 Faremos algumas considerações, neste ponto.

1. Nessa última igualdade do corolário, desprezaremos o termo $\left(\psi_{0}^{-1}\right)^{*} \mu$. Faremos isso porque derivaremos esta igualdade com respeito ao parâmetro $\epsilon$ e este termo não depende de $\epsilon$.

2. Como foi observado no início desta seção, os pontos da órbita de $\psi_{0}$, nessas expressões, estão sobre $\Gamma=G^{-1}(\bar{\Gamma})$, sendo $\Gamma$ uma órbita do Sistema Hamiltoniano bidimensional $X_{H_{1}}$. Devido a essa propriedade, podemos tomar as curvas $\gamma_{j}$, na proposição 7.2.1, como sendo o segmento de órbita de $X_{H_{1}}$, que conecta os pontos $\gamma_{j}(0)=z$ a $\gamma_{j}\left(T_{j}\right)=\psi_{0}^{j}(z)$. Portanto, faremos $\gamma_{j}(t)=\phi_{t}(z)$, com $t \in\left[0, T_{j}\right]$ e $\phi=\phi_{t}^{H_{1}}$.

3. Observemos que devido a estrutura das aplicações de primeiro retorno que estamos analisando, o tempo $T_{j}$ não depende do ponto $z$, depende, apenas, da curva que o contém. Estes tempos de retorno $T_{j}$ serão os mesmos para todo ponto da curva $\Gamma$. Além disso, indicando por $T_{1}$ o período de $\Gamma$, por $X_{H_{1}}$, e por $T_{2}$ o período da órbita corresponde de $X_{H_{2}}$, temos que $T_{j}=j T_{2}$.

Tendo em vistas estas observações, escreveremos:

$$
\bar{M}_{\epsilon}\left(\theta_{z}\right)=\sum_{j=0}^{n-1} \int_{0}^{T_{j}} f_{\epsilon}^{*}(\mu)_{\gamma_{j}(t)}\left(\gamma_{j}^{\prime}(t)\right) d t+n\left(s_{\epsilon} \circ G \circ \psi_{0}^{-1}\right)(z) .
$$


Notemos, ainda, que definindo uma função $g=g_{\epsilon}(x, y)$ como:

$$
g_{\epsilon}(x, y)=\left\{f_{\epsilon}^{*} \mu\right\}_{(x, y)}\left(X_{H_{1}}(x, y)\right) .
$$

Poderemos escrever:

$$
\bar{M}_{\epsilon}\left(\theta_{z}\right)=\sum_{j=0}^{n-1} \int_{0}^{T_{j}} g_{\epsilon}\left(\gamma_{j}(t)\right) d t+n\left(s_{\epsilon} \circ G \circ \psi_{0}^{-1}\right)(z)
$$

O ponto $z$ está sobre a curva $\Gamma$, que é uma trajetória do campo hamiltoniano $X_{H_{1}}$. Fixando um ponto $z_{0} \in \Gamma$, podemos escrever qualquer outro ponto $z \in \Gamma$ como $z=\phi_{s}\left(z_{0}\right)$. Esta observação nos sugere introduzir um parâmetro $s$ para avaliar a igualdade acima. Definimos $M_{\epsilon}(s): \mathbb{R} \rightarrow \mathbb{R}$, dada por:

$$
M_{\epsilon}(s)=\sum_{j=0}^{n-1} \int_{0}^{T_{j}} g_{\epsilon}\left(\phi_{t+s}\left(z_{0}\right)\right) d t+n\left(s_{\epsilon} \circ G \circ \psi_{0}^{-1}\right)\left(\phi_{s}\left(z_{0}\right)\right) .
$$

Observação 7.2.2 Devemos notar que se $\phi_{s}\left(z_{0}\right)=z$ e $G(z)=\left(\theta_{z}, v\left(\theta_{z}\right)\right)$, então $\bar{M}_{\epsilon}\left(\theta_{z}\right)=M_{\epsilon}(s)$. Dessa forma, é possível avaliar o termo, da função de Melnikov, através da função $M_{\epsilon}=M_{\epsilon}(s)$.

Por fim, provaremos duas últimas igualdades relacionadas a essa representação $M_{\epsilon}=M_{\epsilon}(s)$.

Proposição 7.2.2 Vale as seguintes igualdades:

- $M_{1}(s)=\sum_{j=0}^{n-1} \int_{0}^{T_{j}} g_{1}\left(\phi_{t+s}\left(z_{0}\right)\right) d t+n\left(s_{1} \circ G \circ \psi_{0}^{-1}\right)\left(\phi_{s}\left(z_{0}\right)\right)$;

- $M_{1}^{\prime}(s)=\sum_{j=0}^{n-1} g_{1}\left(\phi_{T_{j}+s}\left(z_{0}\right)\right)$

Demonstração: A primeira igualdade é imediata. Observemos que na segunda igualdade, temos:

$$
M_{1}^{\prime}(s)=\sum_{j=0}^{n-1} \int_{0}^{T_{j}} \frac{\partial}{\partial s} g_{1}\left(\phi_{s}\left(\phi_{t}\left(z_{0}\right)\right)\right) d t+\frac{\partial}{\partial s} n\left(s_{1} \circ G \circ \psi_{0}^{-1}\right)\left(\psi_{s}\left(z_{0}\right)\right) .
$$

Vamos dividir a análise nos dois termos do somatório.

1. Temos: 


$$
\begin{aligned}
& \int_{0}^{T_{j}} \frac{\partial}{\partial s} g_{1}\left(\phi_{s}\left(\phi_{t}(z 0)\right)\right) d t=\int_{0}^{T_{j}} d_{\phi_{s+t}\left(z_{0}\right)} g_{1}\left(\frac{\partial}{\partial s}\left(\phi_{s}\left(\phi_{t}\left(z_{0}\right)\right)\right)\right) d t= \\
= & \int_{0}^{T_{j}} d_{\phi_{s+t}\left(z_{0}\right)} g_{1}\left(F\left(\phi_{s+t}\left(z_{0}\right)\right)\right) d t \\
= & \int_{0}^{T_{j}} d_{\phi_{s+t}\left(z_{0}\right)} g_{1}\left(\frac{\partial}{\partial t}\left(\phi_{t}\left(\phi_{s}\left(z_{0}\right)\right)\right)\right) d t \\
= & \int_{0}^{T_{j}} \frac{\partial}{\partial t} g_{1}\left(\phi_{t}\left(\phi_{s}\left(z_{0}\right)\right)\right) d t \\
= & g_{1}\left(\phi_{T_{j}}\left(\phi_{s}\left(z_{0}\right)\right)\right)-g_{1}\left(\phi_{s}\left(z_{0}\right)\right) .
\end{aligned}
$$

Voltando ao somatório, obtemos:

$$
\begin{aligned}
& M_{1}^{\prime}(s)=\sum_{j}\left\{g_{1}\left(\phi_{T_{j}}\left(\phi_{s}\left(z_{0}\right)\right)-g_{1}\left(\phi_{s}\left(z_{0}\right)\right)\right\}+n \frac{\partial}{\partial s}\left(s_{1} \circ G \circ \psi_{0}^{-1}\right)\left(\phi_{s}\left(z_{0}\right)\right)=\right. \\
& =\sum_{j} g_{1}\left(\phi_{T_{j}}\left(\phi_{s}\left(z_{0}\right)\right)\right)+n\left\{\frac{\partial}{\partial s}\left(s_{1} \circ G \circ \psi_{0}^{-1}\right)\left(\phi_{s}\left(z_{0}\right)\right)-g_{1}\left(\phi_{s}\left(z_{0}\right)\right)\right\} .
\end{aligned}
$$

2. Vamos analisar o termo entre chaves.

$$
\begin{aligned}
& \quad \frac{\partial}{\partial s}\left(s_{1} \circ G \circ \psi_{0}^{-1}\right)\left(\phi_{s}\left(z_{0}\right)\right)=\left.\frac{\partial}{\partial s} \frac{\partial}{\partial \epsilon}\right|_{\epsilon=0}\left(s_{\epsilon} \circ G \circ \psi_{0}^{-1}\right)\left(\phi_{s}\left(z_{0}\right)\right) \\
& =\left.\frac{\partial}{\partial \epsilon}\right|_{\epsilon=0} \frac{\partial}{\partial s}\left(s_{\epsilon} \circ G \circ \psi_{0}^{-1}\right)\left(\phi_{s}\left(z_{0}\right)\right)= \\
& =\left.\frac{\partial}{\partial \epsilon}\right|_{\epsilon=0} d_{\phi_{s}\left(z_{0}\right)}\left(s_{\epsilon} \circ G \circ \psi_{0}^{-1}\right)\left(X_{H_{1}}\left(\phi_{s}\left(z_{0}\right)\right)\right) .
\end{aligned}
$$

Mas, nesse ponto, devemos lembrar que:

$d\left(s_{\epsilon} \circ G \circ \psi_{0}^{-1}\right)=f_{\epsilon}^{*} \mu-\left(\psi_{0}^{-1}\right)^{*} \mu$.

Logo:

$$
\begin{aligned}
& \frac{\partial}{\partial s}\left(s_{1} \circ G \circ \psi_{0}^{-1}\right)\left(\phi_{s}\left(z_{0}\right)\right)=\left.\frac{\partial}{\partial \epsilon}\right|_{\epsilon=0}\left(f_{\epsilon}^{*} \mu\right)_{\phi_{s}\left(\theta_{0}\right)}\left(X_{H_{1}}\left(\phi_{s}\left(z_{0}\right)\right)\right)= \\
& =\left.\frac{\partial}{\partial \epsilon}\right|_{\epsilon=0} g_{\epsilon}\left(\phi_{s}\left(z_{0}\right)\right)
\end{aligned}
$$


$=g_{1}\left(\phi_{s}\left(z_{0}\right)\right)$.

Isso nos mostra que o termo entre chaves, acima, é nulo.

Segue o resultado.

Na sequência, faremos o mesmo tipo de análise para o termo $\bar{N}_{1}$.

\subsection{Análise do termo $\bar{N}_{1}$}

Consideremos a função $\xi(x, y)=\bar{\xi}(G(x, y))$, onde $\bar{\xi}(\theta, r)=\lambda_{\varphi_{0}(\theta, r)}\left(\varphi_{1}(\theta, r)\right)$. Teremos:

Lema 7.3.1 Se $f_{\epsilon}$ representa a aplicação, definida na seção anterior, $f_{\epsilon} \doteq \psi_{\epsilon} \circ$ $\psi_{0}^{-1}$, então Vale a igualdade:

$$
\xi(x, y)=\mu_{\psi_{0}(x, y)}\left(f_{1}\left(\psi_{0}(x, y)\right)\right) .
$$

Demonstração: Observemos que:

$$
\xi(x, y)=\lambda_{\varphi_{0}(G(x, y))}\left(\varphi_{1}(G(x, y))\right) .
$$

Seja $\tilde{f}_{\epsilon}$, tal que $\varphi_{\epsilon}=\tilde{f}_{\epsilon} \circ \varphi_{0}$. Então:

$$
\begin{aligned}
\varphi_{1} \circ G=\left.\frac{\partial}{\partial \epsilon}\right|_{\epsilon=0} \tilde{f}_{\epsilon} \circ \varphi_{0} \circ G & =\left.\frac{\partial}{\partial \epsilon}\right|_{\epsilon=0} \tilde{f}_{\epsilon} \circ G \circ \psi_{0}=\left.\frac{\partial}{\partial \epsilon}\right|_{\epsilon=0} G \circ f_{\epsilon} \circ \psi_{0}= \\
& =\left.\frac{\partial}{\partial \epsilon}\right|_{\epsilon=0} G \circ \psi_{\epsilon}
\end{aligned}
$$

Logo: $\psi_{1}(G(x, y))=d_{\psi_{0}(x, y)} G\left(f_{1}\left(\psi_{0}(x, y)\right)\right)$.

Portanto:

$$
\begin{aligned}
& \xi(x, y)=\lambda_{G\left(\psi_{0}(x, y)\right)}\left(d_{\psi_{0}(x, y)} G\left(f_{1}\left(\psi_{0}(x, y)\right)\right)=\left(G^{*} \lambda\right)_{\psi_{0}(x, y)}\left(f_{1}\left(\psi_{0}(x, y)\right)\right)\right. \\
& =\mu_{\psi_{0}(x, y)}\left(f_{1}\left(\psi_{0}(x, y)\right)\right)
\end{aligned}
$$

Com isso, obtemos a igualdade desejada.

Seja $z \in \Gamma$, tal que $G(z)=\left(\theta_{z}, v\left(\theta_{z}\right)\right)$. Então:

$$
\bar{N}_{1}\left(\theta_{z}\right)=\sum_{j=0}^{n-1} \bar{\xi}\left(\theta_{j}, r_{j}\right)=\sum_{j=0}^{n-1} \bar{\xi}\left(G\left(\psi_{0}^{j}(z)\right)\right) \doteq N_{1}(z) .
$$

Segue que: 


$$
N_{1}(z)=\sum_{j=0}^{n-1} \mu_{\psi_{0}\left(x_{j}, y_{j}\right)}\left(f_{1}\left(\psi_{0}\left(x_{j}, y_{j}\right)\right)\right), \text { sendo }\left(x_{j}, y_{j}\right)=\psi_{0}^{j}(z) .
$$

Se definirmos $K(x, y)=\mu(x, y)\left(f_{1}(x, y)\right)$, então :

$$
N_{1}(z)=\sum_{j=0}^{n-1} K\left(x_{j}, y_{j}\right)
$$

Utilizaremos o mesmo argumento que foi utilizado na subseção anterior, para introduzir um parâmetro $s$ na função $M_{\epsilon}=M_{\epsilon}(z)$. Fixemos um ponto $z_{0} \in \Gamma$ e seja $s \in \mathbb{R}$, tal que $z=\phi_{s}\left(z_{0}\right)$, onde $\phi_{s}$ é o fluxo do campo hamiltoniano bidimensional $X_{H_{1}}$. Escrevermos:

$$
\left(x_{j}, y_{j}\right)=\phi_{s+T_{j}}\left(z_{0}\right) \text {, onde } z=\phi_{s}\left(z_{0}\right) .
$$

Portanto, poderemos avaliar o termo $N_{1}=N_{1}(z)$, através da função $N_{1}: \mathbb{R} \rightarrow \mathbb{R}$, dada por:

$$
N_{1}(s)=\sum_{j=0}^{n-1} K\left(\phi_{s+T_{j}}\left(z_{0}\right)\right) .
$$

A derivada dessa função, com respeito a $s$, é dada por:

$$
N_{1}^{\prime}(s)=\sum_{j=0}^{n-1} d_{\phi_{s+T_{j}}\left(z_{0}\right)} K\left(X_{H_{1}}\left(\phi_{T_{j}+s}\left(z_{0}\right)\right)\right) .
$$

A partir dessa representação para as funções $\bar{M}_{1}$ e $\bar{N}_{1}$, voltaremos a análise da função $\bar{L}_{1}$, para que algumas ideias fiquem mais claras.

\subsection{Algumas observações sobre a função $\bar{L}_{1}$}

Vimos que o potencial de Melnikov pode ser calculado por meio de:

$$
\bar{L}_{1}(\theta)=\bar{M}_{1}(\theta)-\bar{N}_{1}(\theta) .
$$

Representamos essas funções, $\bar{M}_{1}$ e $\bar{N}_{1}$, em $\Omega$, cujo domínio é $\mathbb{R}$, a partir de um parâmetro para descrever a curva $\Gamma$, obtendo funções $M_{1}=M_{1}(s)$ e $N_{1}=N_{1}(s)$. Dessa forma, consideramos a nova função:

$$
L_{1}(s)=M_{1}(s)-N_{1}(s) .
$$

A relação entre $L_{1}$ e $\bar{L}_{1}$ se dá da seguinte forma. Se $G\left(\phi_{s}\left(z_{0}\right)\right)=\left(\theta_{s}, v\left(\theta_{s}\right)\right)$, então: 


$$
L_{1}(s)=\bar{L}_{1}\left(\theta_{s}\right)
$$

Portanto poderemos avaliar se $\bar{L}_{1}$ é constante por meio de uma análise de $L_{1}$. Suponhamos, por exemplo, que exista $s_{0} \in \mathbb{R}$, tal que $L_{1}^{\prime}\left(s_{0}\right) \neq 0$. Então, existem $s_{1}, s_{2} \in \mathbb{R}$, próximos de $s_{0}$, tal que $L_{1}\left(s_{1}\right) \neq$ $L_{1}\left(s_{2}\right)$. Portanto, $\bar{L}_{1}\left(\theta_{s_{1}}\right) \neq \bar{L}_{1}\left(\theta_{s_{2}}\right)$ (ou seja, a função $\bar{L}_{1}$ não é constante) Tentaremos avaliar a derivada de $L_{1}$ :

$$
L_{1}^{\prime}(s)=M_{1}^{\prime}(s)-N_{1}^{\prime}(s)
$$

Pela análise feita nos termos $M_{1}$ e $N_{1}$, teremos, então:

$$
\begin{aligned}
& L_{1}^{\prime}(s)=\sum_{j=0}^{n-1}\left\{g_{1}\left(\phi\left(T_{j}+s, z_{0}\right)\right)-d_{\phi\left(T_{j}+s, z_{0}\right)} K\left(X_{H_{1}}\left(\phi\left(T_{j}+s, z_{0}\right)\right)\right)\right\}, \text { onde: } \\
& \text { - } g_{1}=\left.\frac{\partial}{\partial \epsilon}\right|_{\epsilon=0} g_{\epsilon}, \text { sendo } g_{\epsilon}(x, y)=\left(f_{\epsilon}^{*} \mu\right)_{(x, y)}\left(X_{H_{1}}(x, y)\right) . \\
& \text { - } K(x, y)=\mu_{(x, y)}\left(f_{1}(x, y)\right) .
\end{aligned}
$$

Com o objetivo de dar uma melhor expressão para essa função $L_{1}^{\prime}$ (que está no conteúdo do próximo corolário), observemos que o termo correspondente à função $K$, que corresponde ao segundo termo dentro do somatório, também pode ser expresso como uma derivada em $\epsilon$. Seja $K_{\epsilon}(x, y)=$ $\mu_{(x, y)}\left(f_{\epsilon}(x, y)\right)$. Logo $K_{1}=K$ (utilizando a notação fixada). Daí:

$$
\begin{aligned}
& d_{\phi\left(T_{j}+s, z_{0}\right)} K\left(X_{H_{1}}\left(\phi\left(T_{j}+s, z_{0}\right)\right)\right)=\frac{\partial}{\partial s} K_{1}\left(\phi\left(T_{j}+s, z_{0}\right)\right)= \\
& \left.\frac{\partial}{\partial s} \frac{\partial}{\partial \epsilon}\right|_{\epsilon=0} K_{\epsilon}\left(\phi\left(T_{j}+s, z_{0}\right)\right)=\left.\frac{\partial}{\partial \epsilon}\right|_{\epsilon=0} d_{\phi\left(T_{j}+s, z_{0}\right)} K_{\epsilon}\left(X_{H_{1}}\left(\phi\left(T_{j}+s, z_{0}\right)\right)\right) .
\end{aligned}
$$

Logo, com isso obtemos a seguinte igualdade para $L_{1}^{\prime}(s)$ :

$$
L_{1}^{\prime}(s)=\left.\frac{\partial}{\partial \epsilon}\right|_{\epsilon=0} \sum_{j=0}^{n-1}\left\{g_{\epsilon}\left(\phi\left(T_{j}+s, z_{0}\right)\right)-d_{\phi\left(T_{j}+s, z_{0}\right)} K_{\epsilon}\left(X_{H_{1}}\left(\phi\left(T_{j}+s, z_{0}\right)\right)\right)\right\} .
$$

Notemos ainda que a função dentro do somatório pode ser escrita como:

$$
g_{\epsilon}(x, y)-d_{(x, y)} K_{\epsilon}\left(X_{H_{1}}(x, y)\right)=\left(f_{\epsilon}^{*} \mu-d K_{\epsilon}\right)_{(x, y)}\left(X_{H_{1}}(x, y)\right) .
$$

Diante dessas manipulações algébricas, resumiremos a representação obtida para a função $L_{1}^{\prime}(s)$ no próximo corolário.

Corolário 7.4.1 Definindo $A_{\epsilon}(x, y)=\left(f_{\epsilon}^{*} \mu-d K_{\epsilon}\right)_{(x, y)}\left(X_{H_{1}}(x, y)\right)$, obtemos: 


$$
L_{1}^{\prime}(s)=\sum_{j=0}^{n-1} A_{1}\left(\phi\left(T_{j}+s, z_{0}\right)\right) .
$$

Demonstração: Segue dos cálculos feitos acima.

Portanto, se existir $s_{0} \in \mathbb{R}$ tal que $L_{1}^{\prime}\left(s_{0}\right) \neq 0$, então $\bar{L}_{1}$ não é constante e teremos, então, que a curva rotacional invariante $\bar{\Gamma}$ não persisti sob a perturbação. É fundamental notar que o somatório que aparece na expressão de $L_{1}^{\prime}$, no último corolário, é uma soma sobre órbitas de $\psi_{0}$. De fato, através do parâmetro $s$, escolhemos um ponto sobre a curva $\Gamma$; ao variar o índice $j$ do somatório, percorremos a órbita do ponto fixado (por meio de $s$ ) por $\psi_{0}$. Essa construção foi possível devido ao fato de que todo ponto sobre a curva $\Gamma$ possui tempo de retorno, pela aplicação de Poincaré $\psi_{0}$, iguais. $\mathrm{Na}$ sequência do texto, iremos avaliar essa função $A_{1}$, que aparece no corolário anterior.

\subsection{Análise da Função $A_{1}$.}

Vamos analisar melhor esta função $A_{1}$. Conforme foi visto, teremos:

$$
A_{\epsilon}(x, y)=\left(f_{\epsilon}^{*} \mu-d K_{\epsilon}\right)\left(X_{H_{1}}(x, y)\right) .
$$

Escrevermos $f_{\epsilon}(x, y)=\left(X_{\epsilon}(x, y), Y_{\epsilon}(x, y)\right)$. Assim: $K_{\epsilon}(x, y)=\mu_{(x, y)}\left(f_{\epsilon}(x, y)\right)=$ $\mu_{(x, y)}\left(X_{\epsilon}, Y_{\epsilon}\right)$.

Passemos as expressões das 1-formas, que aparecem na expressão de $A_{\epsilon}$, para coordenadas. Seguimos por partes.

1. $f_{\epsilon}^{*} \mu$.

Observemos que calculando essa 1-forma num vetor $v \in T_{(x, y)} \mathbb{R}^{2}$, temos:

$$
\begin{aligned}
& \left(f_{\epsilon}^{*} \mu\right)_{(x, y)}(v)=\mu_{f_{\epsilon}(x, y)}\left(d_{(x, y)} f_{\epsilon}(v)\right)=\mu_{\left(X_{\epsilon}, Y_{\epsilon}\right)}\left(d_{(x, y)} f_{\epsilon}(v)\right)= \\
& =\frac{X_{\epsilon}}{2} d \pi_{2}\left(\left(d_{(x, y)} f_{\epsilon}(v)\right)\right)-\frac{Y_{\epsilon}}{2} d \pi_{1}\left(\left(d_{(x, y)} f_{\epsilon}(v)\right)\right) .
\end{aligned}
$$

Onde, as aplicações $\pi_{i}, i=1$ ou $i=2$, representam as projeções canônicas. Segue que:

$$
\left(f_{\epsilon}^{*} \mu\right)_{(x, y)}(v)=\frac{X_{\epsilon}}{2} d_{(x, y)} Y_{\epsilon}(v)-\frac{Y_{\epsilon}}{2} d_{(x, y)} X_{\epsilon}(v)=
$$


Portanto, podemos escrever

$$
\left(f_{\epsilon}^{*} \mu\right)_{(x, y)}=\left(\frac{X_{\epsilon}}{2} \frac{\partial Y_{\epsilon}}{\partial x}-\frac{Y_{\epsilon}}{2} \frac{\partial X_{\epsilon}}{\partial x}\right) d x+\left(\frac{X_{\epsilon}}{2} \frac{\partial Y_{\epsilon}}{\partial y}-\frac{Y_{\epsilon}}{2} \frac{\partial X_{\epsilon}}{\partial y}\right) d y
$$

2. $d K_{\epsilon}$

Faremos o mesmo com $d K_{\epsilon}$. Como $K_{\epsilon}(x, y)=\mu_{(x, y)}\left(X_{\epsilon}, Y_{\epsilon}\right)$, temos:

$$
K_{\epsilon}(x, y)=\frac{x}{2} d y\left(X_{\epsilon}, Y_{\epsilon}\right)-\frac{y}{2} d x\left(X_{\epsilon}, Y_{\epsilon}\right)=\frac{x}{2} Y_{\epsilon}-\frac{y}{2} X_{\epsilon}
$$

Segue então que:

$$
\frac{\partial K_{\epsilon}}{\partial x}=\frac{Y_{\epsilon}}{2}+\frac{x}{2} \frac{\partial Y_{\epsilon}}{\partial x}-\frac{y}{2} \frac{\partial X_{\epsilon}}{\partial x} \mathrm{e} \frac{\partial K_{\epsilon}}{\partial y}=\frac{x}{2} \frac{\partial Y_{\epsilon}}{\partial y}-\frac{y}{2} \frac{X_{\epsilon}}{\partial y}-\frac{X_{\epsilon}}{2}
$$

Portanto, podemos escrever, em relação à base $\{d x, d y\}$ :

$$
d K_{\epsilon}=\left(\frac{x}{2} \frac{\partial Y_{\epsilon}}{\partial x}-\frac{y}{2} \frac{\partial X_{\epsilon}}{\partial x}+\frac{Y_{\epsilon}}{2}\right) d x+\left(\frac{x}{2} \frac{\partial Y_{\epsilon}}{\partial y}-\frac{y}{2} \frac{\partial X_{\epsilon}}{\partial y}-\frac{X_{\epsilon}}{2}\right) d y
$$

3. $\left(f_{\epsilon}^{*} \mu-d K_{\epsilon}\right)$

Juntando as duas expressões obtidas, podemos escrever:

$$
\begin{array}{r}
\left(f_{\epsilon}^{*} \mu-d K_{\epsilon}\right)=B_{\epsilon}(x, y) d x+C_{\epsilon}(x, y) d y, \text { onde: } \\
\left\{\begin{array}{l}
B_{\epsilon}(x, y)=\frac{X_{\epsilon}}{2} \frac{\partial Y_{\epsilon}}{\partial x}-\frac{Y_{\epsilon}}{2} \frac{\partial X_{\epsilon}}{\partial x}-\frac{x}{2} \frac{\partial Y_{\epsilon}}{\partial x}+\frac{y}{2} \frac{\partial X_{\epsilon}}{\partial x}-\frac{Y_{\epsilon}}{2} \\
C_{\epsilon}(x, y)=\frac{X_{\epsilon}}{2} \frac{\partial Y_{\epsilon}}{\partial y}-\frac{Y_{\epsilon}}{2} \frac{\partial X_{\epsilon}}{\partial y}-\frac{x}{2} \frac{\partial Y_{\epsilon}}{\partial y}+\frac{y}{2} \frac{\partial X_{\epsilon}}{\partial y}-\frac{X_{\epsilon}}{2}
\end{array}\right.
\end{array}
$$

Com isso, obtemos uma boa representação para a 1-forma que aparece na expressão da função $A_{\epsilon}$.

Vamos, agora, avaliar as derivadas, em relação ao parâmetro $\epsilon$, das funções obtidas $B_{\epsilon}$ e $C_{\epsilon}$. Antes disso, faremos uma observação.

Observação 7.5.1 Escrevendo $f_{\epsilon}(x, y)=\left(X_{\epsilon}(x, y), Y_{\epsilon}(x, y)\right)$, devemos notar que:

$$
X_{\epsilon}(0,0)=0 \text { e } Y_{\epsilon}(0,0)=0, \forall \epsilon .
$$


Isso é consequência de $\psi_{\epsilon}(0,0)=(0,0), \forall \epsilon$ (ou seja, a órbita periódica é a mesma, quando variamos o parâmetro). Portanto:

$$
X_{1}(0,0)=0 \text { e } Y_{1}(0,0)=0
$$

Por outro lado, devemos notar que $f_{0}=I d$ (é a identidade). Logo:

$$
X_{0}(x, y)=x \text { e } Y_{0}(x, y)=y
$$

Essas relações serão importantes nos próximos cálculos.

Vamos aos cálculos de $B_{1}$ e $C_{1}$. Seguiremos, por partes.

1. Cálculo da função $B_{1}$.

Temos:

$$
B_{\epsilon}(x, y)=\frac{X_{\epsilon}}{2} \frac{\partial Y_{\epsilon}}{\partial x}-\frac{Y_{\epsilon}}{2} \frac{\partial X_{\epsilon}}{\partial x}-\frac{x}{2} \frac{\partial Y_{\epsilon}}{\partial x}+\frac{y}{2} \frac{\partial X_{\epsilon}}{\partial x}-\frac{Y_{\epsilon}}{2}
$$

Vamos, a seguir, derivar, em relação a $\epsilon$, cada termo que aparece na soma dessa expressão:

- $\left.\frac{\partial}{\partial \epsilon}\right|_{\epsilon=0}\left(\frac{X_{\epsilon}}{2} \frac{\partial Y_{\epsilon}}{\partial x}\right)=\frac{X_{1}}{2} \frac{\partial Y_{0}}{\partial x}+\frac{X_{0}}{2} \frac{\partial Y_{1}}{\partial x}=\frac{x}{2} \frac{\partial Y_{1}}{\partial x}$.

- $\left.\frac{\partial}{\partial \epsilon}\right|_{\epsilon=0}\left(\frac{Y_{\epsilon}}{2} \frac{\partial X_{\epsilon}}{\partial x}\right)=\frac{Y_{1}}{2} \frac{\partial X_{0}}{\partial x}+\frac{Y_{0}}{2} \frac{\partial X_{1}}{\partial x}=\frac{Y_{1}}{2}+\frac{y}{2} \frac{\partial X_{1}}{\partial x}$.

- $\left.\frac{\partial}{\partial \epsilon}\right|_{\epsilon=0}\left(\frac{x}{2} \frac{\partial Y_{\epsilon}}{\partial x}\right)=\frac{x}{2} \frac{\partial Y_{1}}{\partial x}$.

- $\left.\frac{\partial}{\partial \epsilon}\right|_{\epsilon=0}\left(\frac{y}{2} \frac{\partial X_{\epsilon}}{\partial x}\right)=\frac{y}{2} \frac{\partial X_{1}}{\partial x}$.

- $\left.\frac{\partial}{\partial \epsilon}\right|_{\epsilon=0}\left(\frac{Y_{\epsilon}}{2}\right)=\frac{Y_{1}}{2}$.

Tendo em vista os sinais na expressão, acima, de $B_{\epsilon}$, e essas derivadas com respeito a $\epsilon$, concluímos que:

$$
B_{1}(x, y)=-\frac{Y_{1}}{2}-\frac{Y_{1}}{2}=-Y_{1}(x, y) .
$$

2. Cálculo da função $C_{1}$.

Esquematizaremos o cálculo de $C_{1}$ da mesma forma que fizemos com $B_{1}$. Temos: 


$$
C_{\epsilon}(x, y)=\frac{X_{\epsilon}}{2} \frac{\partial Y_{\epsilon}}{\partial y}-\frac{Y_{\epsilon}}{2} \frac{\partial X_{\epsilon}}{\partial y}-\frac{x}{2} \frac{\partial Y_{\epsilon}}{\partial y}+\frac{y}{2} \frac{\partial X_{\epsilon}}{\partial y}-\frac{X_{\epsilon}}{2}
$$

Vamos derivar, em relação a $\epsilon$, cada termo que aparece nessa expressão:

$$
\begin{aligned}
& \text { - }\left.\frac{\partial}{\partial \epsilon}\right|_{\epsilon=0}\left(\frac{X_{\epsilon}}{2} \frac{\partial Y_{\epsilon}}{\partial y}\right)=\frac{X_{1}}{2} \frac{\partial Y_{0}}{\partial y}+\frac{X_{0}}{2} \frac{\partial Y_{1}}{\partial y}=\frac{X_{1}}{2}+\frac{x}{2} \frac{\partial Y_{1}}{\partial y} . \\
& \text { - }\left.\frac{\partial}{\partial \epsilon}\right|_{\epsilon=0}\left(\frac{Y_{\epsilon}}{2} \frac{\partial X_{\epsilon}}{\partial y}\right)=\frac{Y_{1}}{2} \frac{\partial X_{0}}{\partial y}+\frac{Y_{0}}{2} \frac{\partial X_{1}}{\partial y}=\frac{y}{2} \frac{\partial X_{1}}{\partial y} . \\
& \text { - }\left.\frac{\partial}{\partial \epsilon}\right|_{\epsilon=0}\left(\frac{x}{2} \frac{\partial Y_{\epsilon}}{\partial y}\right)=\frac{x}{2} \frac{\partial Y_{1}}{\partial y} . \\
& \text { - }\left.\frac{\partial}{\partial \epsilon}\right|_{\epsilon=0}\left(\frac{y}{2} \frac{\partial X_{\epsilon}}{\partial y}\right)=\frac{y}{2} \frac{\partial X_{1}}{\partial y} . \\
& \text { - }\left.\frac{\partial}{\partial \epsilon}\right|_{\epsilon=0}\left(\frac{X_{\epsilon}}{2}\right)=\frac{X_{1}}{2} .
\end{aligned}
$$

Tendo em vista os sinais na expressão de $C_{\epsilon}$ e essas derivadas, obtemos:

$$
C_{1}(x, y)=\frac{X_{1}}{2}+\frac{X_{1}}{2}=X_{1}(x, y)
$$

Diante dessa representação de $B_{1}$ e $C_{1}$, conseguimos a seguinte igualdade para $A_{1}=A_{1}(x, y)$.

Proposição 7.5.1 Se $V$ é o potencial ${ }^{2}$ do Sistema Hamiltoniano $H_{1}$ (que foi indicado, na seção 3, por $\left.V_{1}\right)$, temos:

$$
A_{1}(x, y)=-y Y_{1}(x, y)-V^{\prime}(x) X_{1}(x, y) .
$$

Demonstração: Observemos que:

$$
A_{\epsilon}(x, y)=\left(f_{\epsilon}^{*} \mu-d K_{\epsilon}\right)_{(x, y)}\left(X_{H_{1}}(x, y)\right)
$$

Como $X_{H_{1}}(x, y)=\left(y,-V^{\prime}(x)\right) e\left(f_{\epsilon}^{*} \mu-d K_{\epsilon}\right)=B_{\epsilon}(x, y) d x+C_{\epsilon}(x, y) d y$, temos:

$$
A_{\epsilon}(x, y)=B_{\epsilon} d x\left(y,-V^{\prime}(x)\right)+C_{\epsilon} d y\left(y,-V^{\prime}(x)\right)=y B_{\epsilon}(x, y)-V^{\prime}(x) C_{\epsilon}(x, y) .
$$

Derivando, com respeito a $\epsilon$, esta igualdade:

\footnotetext{
${ }^{2}$ Iremos indicá-lo por $V$ e não por $V_{1}$, conforme introduzido no texto. Faremos assim, para não confundir com a notação que representa a derivada com relação ao parâmetro $\epsilon$.
} 


$$
A_{1}(x, y)=y B_{1}(x, y)-V^{\prime}(x) C_{1}(x, y) .
$$

Utilizando as igualdades obtidas nos cálculos acima, $B_{1}=-Y_{1}$ e $C_{1}=X_{1}$, conseguimos:

$$
A_{1}(x, y)=-y Y_{1}(x, y)-V^{\prime}(x) X_{1}(x, y) .
$$

Obtemos, assim, a igualdade desejada.

Portanto, essa função $A_{1}(x, y)=-y Y_{1}(x, y)-V^{\prime}(x) X_{1}(x, y)$ que deveremos avaliar a soma sobre as órbitas de $\psi_{0}$ em $\Gamma$, conforme vem sendo discutido nesta seção. Tendo em vista que essa curva $\Gamma$ é tomada próxima a origem $^{3}(0,0) \in \Gamma$ (ou seja, $\Gamma$ próxima ao ponto fixo), vamos avaliar, agora, como essa função $A_{1}$ se comporta próximo ao ponto fixo, $(0,0) \in \Omega$, ou seja, iremos calcular $A_{1}$ e suas primeiras derivadas em $(0,0)$ (essas informações estão resumidas na próxima proposição).

Observemos que $A_{1}(0,0)=0$. Além disso:

$$
\left\{\begin{array}{l}
\frac{\partial A_{1}}{\partial x}=-y \frac{\partial Y_{1}}{\partial x}-V^{\prime \prime} X_{1}-V^{\prime} \frac{\partial X_{1}}{\partial x} \\
\frac{\partial A_{1}}{\partial y}=-Y_{1}-y \frac{\partial Y_{1}}{\partial y}-V^{\prime} \frac{\partial X_{1}}{\partial y} .
\end{array}\right.
$$

Temos ainda que $X_{1}(0,0)=0$ e $Y_{1}(0,0)=0$. Logo:

$$
\frac{\partial A_{1}}{\partial x}(0,0)=0 \text { e } \frac{\partial A_{1}}{\partial y}(0,0)=0 .
$$

Vamos avaliar, agora, as derivadas de ordem 2 de $A_{1}$ em $(0,0)$. Observemos que a partir das igualdades (7.3), obtemos:

- $\frac{\partial^{2} A_{1}}{\partial x^{2}}(0,0)=-V^{\prime \prime}(0) \frac{\partial X_{1}}{\partial x}(0,0)-V^{\prime \prime} \frac{\partial X_{1}}{\partial x}(0,0)=-2 \alpha^{2} \frac{\partial X_{1}}{\partial x}(0,0)$.

- $\frac{\partial^{2} A_{1}}{\partial y^{2}}(0,0)=-\frac{\partial Y_{1}}{\partial y}(0,0)-\frac{\partial Y_{1}}{\partial y}(0,0)=-2 \frac{\partial Y_{1}}{\partial y}(0,0)$.

- $\frac{\partial^{2} A_{1}}{\partial x \partial y}(0,0)=-\frac{\partial Y_{1}}{\partial x}(0,0)-V^{\prime \prime}(0) \frac{\partial X_{1}}{\partial y}(0,0)=-\frac{\partial Y_{1}}{\partial x}-\alpha^{2} \frac{\partial X_{1}}{\partial y}$.

Portanto, "as primeiras derivadas" da função $A_{1}$ que podem ser não nulas em $(0,0)$ são as derivadas de ordem 2 e estão relacionadas às derivadas de ordem 1 das funções $X_{1}$ e $Y_{1}$ em $(0,0)$. Essas funções, ou suas derivadas, estão relacionadas às propriedades dinâmicas do campo $X_{H_{\epsilon}}$, conforme analisaremos na próxima seção. Resumiremos o comportamento local, provado nos argumentos acima, da função $A_{1}$, na próxima proposição.

${ }^{3}$ Ver a observação 7.1.1. 
Proposição 7.5.2 Temos que a função $A_{1}$ se anula em $(0,0)$, juntamente com suas derivadas de ordem 1. Suas derivadas de ordem 2 são dadas por:

- $\frac{\partial^{2} A_{1}}{\partial x^{2}}(0,0)=-2 \alpha^{2} \frac{\partial X_{1}}{\partial x}(0,0)$.

- $\frac{\partial^{2} A_{1}}{\partial y^{2}}(0,0)=-2 \frac{\partial Y_{1}}{\partial y}(0,0)$.

- $\frac{\partial^{2} A_{1}}{\partial x \partial y}(0,0)=-\frac{\partial Y_{1}}{\partial x}-\alpha^{2} \frac{\partial X_{1}}{\partial y}$

Observação 7.5.2 Diante da proposição anterior e dos comentários relacionados, podemos escrever, então, $A_{1}(x, y)=Q(x, y)+R(x, y)$, onde $Q=$ $Q(x, y)$ é a forma quadrática associada a hessiana de $A_{1}$ em $(0,0)$ e $R=$ $R(x, y)$ é o termo de resto. Assim, teremos:

$$
\lim _{(x, y) \rightarrow(0,0)} \frac{R(x, y)}{\|(x, y)\|^{2}}=0
$$

Portanto, em uma vizinhança de $(0,0) \in \mathbb{R}^{2}$ suficientemente pequena, o termo $R=R(x, y)$ contribui pouco com o valor de $A_{1}$, ou melhor, o termo $Q=Q(x, y)$ predomina. Estamos interessados em avaliar a soma da função $A_{1}$ sobre órbitas da aplicação $\psi_{0}$, órbitas que estão sobre a curva invariante $\Gamma$. Se a curva invariante $\Gamma$ estiver suficientemente próxima a origem $(0,0) \in \Omega$, então o valor do termo $Q$, sobre pontos da órbitas da função $\psi_{0}$, estará "muito próxima"do respectivo valor da soma da função $A_{1}$. Essa é a ideia para fazer a análise, que será feita mais a frente.

\subsection{As Derivadas da Função $A_{1}$.}

Tendo em vista a observação 7.5.2, temos que calcular as derivadas de segunda ordem da função $A_{1}$ em $(0,0)$. Isso nos permite trabalhar com a forma quadrática $Q=Q(x, y)$. Esses cálculos estão relacionados ao fluxo do campo Hamiltoniano $X_{H_{\epsilon}}$. Os resultados finais de tais cálculos estão na próxima proposição. Uma exposição completa destes cálculos está no Apêndice A.

Proposição 7.6.1 Indicando a órbita $\Gamma_{E}$, como no Lema 1.1.1, por $\Gamma_{E}(t)=$ $\left(0, x_{2}(t), 0, y_{2}(t)\right)$, sendo $T$ o seu período. As derivadas parciais da função $A_{1}$, de segunda ordem, são: 
- $\frac{\partial^{2} A_{1}}{\partial x \partial y}(0,0)=2 \int_{0}^{T} x_{2}^{2}(s) \cos \{2 \alpha(T-s)\} d s$

- $\frac{\partial^{2} A_{1}}{\partial y^{2}}(0,0)=\frac{2}{\alpha} \int_{0}^{T} x_{2}^{2}(s) \sin \{2 \alpha(T-s)\} d s$;

- $\frac{\partial^{2} A_{1}}{\partial x^{2}}(0,0)=2 \alpha \int_{0}^{T} x_{2}^{2}(s) \sin \{2 \alpha(T-s)\} d s$

Observemos que as derivadas de segunda ordem de $A_{1}$, em $(0,0)$, dependem, basicamente, do parâmetro $\alpha$ e de integrais sobre a órbita periódica $\Gamma_{E}$.

Nesse ponto, podemos escrever a função $Q=Q(x, y)$, que foi descrita na observação 7.5.2, por:

$$
Q(x, y)=a x^{2}+2 c x y-b y^{2}
$$

Onde:

$$
a=\frac{\partial^{2} A_{1}}{\partial x^{2}}(0,0), c=\frac{\partial^{2} A_{1}}{\partial x \partial y}(0,0) \text { e } b=-\frac{\partial^{2} A_{1}}{\partial y^{2}}(0,0)
$$

$\mathrm{Na}$ próxima seção, trataremos de melhorar a expressão da função $L_{1}^{\prime}=$ $L_{1}^{\prime}(s)$, escrevendo explicitamente as soluções do Sistema Hamiltoniano bidimensional $H_{1}$. 


\section{Capítulo 8}

\section{Destruição de Curvas Invariantes.}

O objetivo deste capítulo é provar o Teorema 1.1.3, a partir da condição de Melnikov que foi estabelecida, no capítulo anterior, nas coordenadas da seção de Poincaré $\Sigma_{E}^{+}$.

\subsection{A condição de Melnikov a partir das soluções explícitas de $X_{H_{1}}$.}

Como vem sendo destacado no texto, gostaríamos de avaliar a funçãoํㅜㄹ

$$
L \doteq L_{1}^{\prime}: \mathbb{R} \rightarrow \mathbb{R}
$$

Dada por: $L(s)=\sum_{j=0}^{n-1} A_{1}\left(\phi\left(s+T_{j}\right), z_{0}\right)$,

onde $z_{0} \in \Gamma$, $z_{0}$ está fixado e $\Gamma$ é a curva $(m, n)$-ressonante, a qual queremos avaliar se persiste ou não, e $\phi=\phi(t, z)$ é o fluxo do Campo Hamiltoniano bidimensional $X_{H_{1}}$.

Vale ressaltar que indicando por $T_{1}$ o período da órbita de $\Gamma$ (órbita de $X_{H_{1}}$ ) e por $T_{2}$ o correspondente período no sistema hamiltoniano $H_{2}$, teremos:

$$
\frac{T_{2}}{T_{1}}=\frac{m}{n}
$$

onde $m, n \in \mathbb{N}$ são primos entre si. Dessa forma, teremos, na expressão da função $L=L(s), T_{j}=j T_{2}$.

\footnotetext{
${ }^{1}$ Ao longo dessa seção, indicaremos a função $L_{1}^{\prime}$ simplesmente por $L$
} 
Se a função $L=L(s)$ não for identicamente nula, então a curva $\Gamma$ não persiste sob a perturbação (no sentido da definição (6.1.1)). "Para uma melhor análise dessa questão", iremos, nessa seção, "explicitar" o fluxo $s \mapsto$ $\phi\left(s, z_{0}\right)$, que corresponderá a uma parametrização da curva $\Gamma$. A equação de Hamilton, associada a Hamiltoniana $H_{1}$, é dada por:

$$
\left\{\begin{array}{l}
q^{\prime}=p \\
p^{\prime}=-\alpha^{2} q-2 q^{3}
\end{array}\right.
$$

(Estamos supondo, sem perda de generalidade, que o coeficiente $b_{1}=1$.)

Sendo $\Gamma=H_{1}^{-1}\left(E_{1}\right)$, temos:

$$
\frac{p^{2}}{2}+\frac{\alpha^{2} q^{2}}{2}+\frac{q^{4}}{2}=E_{1}
$$

Segue então que:

$$
\frac{d q}{d t}=\sqrt{2 E_{1}-\alpha^{2} q^{2}-q^{4}}
$$

No entanto, considerando o polinômio dentro da raíz, podemos escrever:

$$
2 E_{1}-\alpha^{2} x^{2}-x^{4}=\left(r_{1}-x^{2}\right)\left(r_{2}+x^{2}\right)
$$

Onde $r_{1}$ e $r_{2}$ são dados por:

$$
\left\{\begin{array}{l}
r_{1}=\frac{-\alpha^{2}+\sqrt{\alpha^{4}+8 E_{1}}}{2} \\
r_{2}=\frac{\alpha^{2}+\sqrt{\alpha^{4}+8 E_{1}}}{2}
\end{array}\right.
$$

Teremos a situação indicada na figura 8.1.

Além disso:

$$
\int_{t}^{\frac{T_{1}}{4}} d t=\int_{q}^{\sqrt{r_{1}}} \frac{d x}{\sqrt{\left(r_{1}-x^{2}\right)\left(r_{2}+x^{2}\right)}}
$$

Sendo $q \in\left[0, \sqrt{r_{1}}\right]$ a coordenada no instante $t, t \in\left[0, \frac{T_{1}}{4}\right]$.

A integral do lado direito é chamada integral elíptica (ver ([17])). Podemos expressar esse tipo de integral a partir das chamadas funções elípticas de Jacobi (suas inversas). Integrais, como a apresentada acima, satisfazem:

$$
\int_{x}^{b} \frac{d t}{\sqrt{\left(b^{2}-t^{2}\right)\left(a^{2}+t^{2}\right)}}=\frac{1}{\sqrt{a^{2}+b^{2}}} c n^{-1}\left(\frac{x}{b}\right)
$$




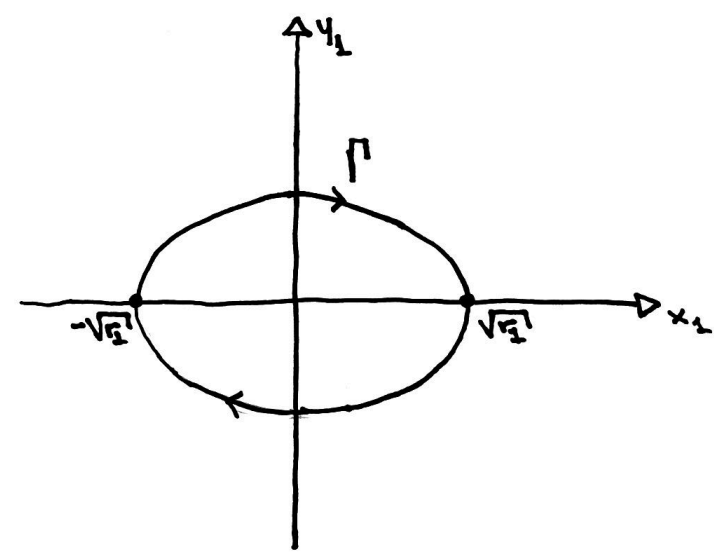

Figura 8.1: Representação da curva $\Gamma$.

onde cn é a função cosseno elíptico e o módulo da função cosseno elíptico, contido nessa expressão, é dado por:

$$
k=\frac{b}{\sqrt{a^{2}+b^{2}}}
$$

Essas informações estão em [17]. No nosso caso, teremos $r_{1}=b^{2}$ e $r_{2}=a^{2}$. Logo:

$$
\frac{T_{1}}{4}-t=\frac{1}{\sqrt{r_{1}+r_{2}}} c n^{-1}\left(\frac{q}{\sqrt{r_{1}}}\right)
$$

Onde o módulo da função elíptica é dado por:

$$
k=\sqrt{\frac{r_{1}}{r_{1}+r_{2}}}
$$

Portanto:

$$
c n^{-1}\left(\frac{q}{\sqrt{r_{1}}}\right)=\sqrt{r_{1}+r_{2}}\left(\frac{T_{1}}{4}-t\right) \Longrightarrow q(t)=\sqrt{r_{1}} c n\left(\sqrt{r_{1}+r_{2}}\left(\frac{T_{1}}{4}-t\right)\right)
$$

Isso nos sugere poder expressar as soluções da equação de Hamilton associada a função $H_{1}$ por meio das funções elípticas de Jacobi. Verificaremos, 
a seguir, que a função $q: \mathbb{R} \longrightarrow \mathbb{R}$, dada por $q(t)=\sqrt{r_{1}} c n\left(\sqrt{r_{1}+r_{2}} t\right)$, onde o módulo da função elíptica é dado por $k=\sqrt{\frac{r_{1}}{r_{1}+r_{2}}}$, é solução da seguinte equação:

$$
\left\{\begin{array}{l}
x^{\prime \prime}=-\alpha^{2} x-2 x^{3} \\
x(0)=\sqrt{r_{1}} \\
x^{\prime}(0)=0
\end{array}\right.
$$

As funções elípticas de Jacobi estão relacionadas a certas equações diferenciais. Em relação a isso, temos:

Lema 8.1.1 Seja $y(x)=c n(x)$, onde cn é o cosseno elíptico, associado ao módulo k. Então vale:

$$
\frac{d^{2} y}{d x^{2}}=\left(2 k^{2}-1\right) y-2 k^{2} y^{3}
$$

Demonstração: $\operatorname{Ver}([17])$.

Diante desse lema, obtemos o seguinte corolário:

Corolário 8.1.1 A função $q: \mathbb{R} \rightarrow \mathbb{R}$, dada por:

$$
q(t)=\sqrt{r_{1}} \operatorname{cn}\left(\sqrt{r_{1}+r_{2}} t\right) \text {, com módulo } k=\sqrt{\frac{r_{1}}{r_{1}+r_{2}}}
$$

é solução de (8.4). Onde $r_{1}$ e $r_{2}$ são dados em (8.2).

Demonstração: Observemos que pela definição da função $q=q(t)$, teremos:

$$
\frac{q(t)}{\sqrt{r_{1}}}=c n\left(\sqrt{r_{1}+r_{2}} t\right) \Longrightarrow \frac{q^{\prime}(t)}{\sqrt{r_{1}}}=\left(\sqrt{r_{1}+r_{2}}\right) c n^{\prime}\left(\sqrt{r_{1}+r_{2}} t\right) .
$$

Derivando mais uma vez, obtemos:

$$
\frac{q^{\prime \prime}(t)}{\sqrt{r_{1}}}=\left(r_{1}+r_{2}\right) c n^{\prime \prime}\left(\left(\sqrt{r_{1}+r_{2}}\right) t\right)
$$

Por outro lado, teremos:

$$
r_{1}-r_{2}=\alpha^{2} \Longrightarrow r_{2}=r_{1}+\alpha^{2} .
$$

Segue que:

$$
2 k^{2}-1=\frac{2 r_{1}}{r_{1}+r_{2}}-1=\frac{2 r_{1}-r_{1}-r_{2}}{r_{1}+r_{2}}=\frac{r_{1}-r_{2}}{r_{1}+r_{2}} .
$$

Obtemos então: 


$$
2 k^{2}-1=\frac{-\alpha^{2}}{r_{1}+r_{2}} e 2 k^{2}=\frac{2 r_{1}}{r_{1}+r_{2}} .
$$

Substituindo na igualdade que nos dá $q^{\prime \prime}$, obtemos:

$$
\frac{q^{\prime \prime}(t)}{\sqrt{r_{1}}}=\left(r_{1}+r_{2}\right)\left(\frac{-\alpha^{2}}{\sqrt{r_{1}}} \frac{q(t)}{r_{1}+r_{2}}-\frac{2 r_{1}}{\sqrt{r_{1}}} \frac{q^{3}(t)}{r_{1}+r_{2}}\right)=\frac{-\alpha^{2} q(t)}{\sqrt{r_{1}}}-\frac{2}{\sqrt{r_{1}}} q^{3}(t) .
$$

Portanto:

$$
q^{\prime \prime}(t)=-\alpha^{2} q(t)-2 q^{3}(t)
$$

Devemos notar ainda que $q(0)=\sqrt{r_{1}}$. Além disso, vale:

$$
q^{\prime}(t)=-\left(\sqrt{r_{1}^{2}+r_{1} r_{2}}\right) \operatorname{sn}\left(\left(\sqrt{r_{1}+r_{2}}\right) t\right) d n\left(\left(\sqrt{r_{1}+r_{2}}\right) t\right)
$$

Logo, $q^{\prime}(0)=0$. Obtemos, assim, que $q=q(t)$ é solução de (8.4).

Definimos, a partir deste corolário, a seguinte curva:

$$
z: \mathbb{R} \rightarrow \mathbb{R}^{2}, z(t)=(q(t), p(t))
$$

Onde as curvas $q=q(t)$ e $p=p(t)$ são dados por:

$$
\left\{\begin{array}{l}
q(t)=\sqrt{r_{1}} \operatorname{cn}\left(\sqrt{\left(r_{1}+r_{2}\right)} t\right) \\
p(t)=-\left(\sqrt{r_{1}^{2}+r_{1} r_{2}}\right) \operatorname{sn}\left(\left(\sqrt{r_{1}+r_{2}}\right) t\right) d n\left(\left(\sqrt{r_{1}+r_{2}}\right) t\right)
\end{array}\right.
$$

Diante dessa definição, obtemos:

Corolário 8.1.2 Se $z_{0}=\left(\sqrt{r_{1}}, 0\right)$, então:

$$
\phi\left(s, z_{0}\right)=z(s) .
$$

Onde $\phi$ é o fluxo do campo hamiltoniano $X_{H_{1}}$.

Portanto, fazendo, na expressão de $L=L(s), z_{0}=\left(\sqrt{r_{1}}, 0\right)$, obtemos:

$$
L(s)=\sum_{j=0}^{n-1} A_{1}\left(z\left(s+j \cdot T_{2}\right)\right)
$$

Nesse caso, a curva $s \mapsto \phi\left(s, z_{0}\right)$, associada à expressão de $L=L(s)$ está explícita, expressa através das funções elípticas de Jacobi.

A função $A_{1}=A_{1}(x, y)$ é da forma $A_{1}(x, y)=Q(x, y)+R(x, y)$, sendo a função $Q$ da forma: 


$$
Q(x, y)=a x^{2}+2 c x y-b y^{2}
$$

Onde os coeficientes são dados em (7.4). A função $R=R(x, y)$, por sua vez, satisfaz:

$$
\lim _{(x, y) \rightarrow(0,0)} \frac{R(x, y)}{\|(x, y)\|^{2}}=0
$$

Definiremos as funções $\mu_{1}: \mathbb{R} \rightarrow \mathbb{R}$ e $\mu_{2}: \mathbb{R} \rightarrow \mathbb{R}$, dadas por:

$$
\mu_{1}(s)=Q(z(s)) \text { e } \mu_{2}(s)=R(z(s)) .
$$

Assim:

$$
L(s)=\sum_{j=0}^{n-1}\left\{\mu_{1}\left(s+j \cdot T_{2}\right)+\mu_{2}\left(s+j \cdot T_{2}\right)\right\}
$$

Gostaríamos de mostrar que a função $L=L(s)$ não é identicamente nula. Em particular, se $L$ não for uma função constante, então teremos que $L$ não é identicamente nula. Uma técnica, para analisar esse tipo de soma, vem da análise complexa. De fato, as funções $u \mapsto c n(u), u \mapsto s n(u)$ e $u \mapsto d n(u)$ (assim como as demais funções elípticas) se estendem, analiticamente, a valores do plano complexos; a partir disso, podemos pensar num mesmo tipo de extensão para a função $L$. Essas funções, vistas como funções definidas no plano complexo, serão funções meromorfas duplamente periódicas. Uma análise das singularidades complexas desse tipo de função pode nos levar a concluir se elas são constantes ou não. Seguiremos nessa direção. Os argumentos utilizados aqui são adaptações dos argumentos feitos em [9]. Na sequência do texto, vamos analisar o termo do somatório relacionado a função $\mu_{1}$, considerando as funções elípticas definidas no plano complexo, e tentaremos estudar as singularidades da função obtida.

\subsection{Análise do termo relacionado a $\mu_{1}$}

Como foi definido acima, a função $\mu_{1}=\mu_{1}(s)$ é dada por:

$$
\mu_{1}(s)=Q(z(s)) \text {, onde } Q(x, y)=a x^{2}+2 c x y-b y^{2}
$$

Os coeficientes $a, b$ e $c$ são dados em (7.4). Em particular, teremos:

$$
a=2 \alpha \int_{0}^{T} x_{2}^{2}(s) \sin \{2 \alpha(T-s)\} d s \mathrm{e}
$$




$$
b=\frac{2}{\alpha} \int_{0}^{T} x_{2}^{2}(s) \sin \{2 \alpha(T-s)\} d s
$$

Trabalharemos com a seguinte hipótese, relacionada ao nível de energia:

Hipótese 8.2.1 Vamos supor que coeficiente $\alpha$ e o nível de energia, que é utilizado na construção da transformação de Poincaré, são tais que a seguinte condição é satisfeita:

$$
\int_{0}^{T} x_{2}^{2}(s) \sin \{2 \alpha(T-s)\} d s \neq 0
$$

Devemos destacar que, fixado o coeficiente $\alpha$ da Hamiltoniana, essa é uma hipótese sobre o nível de energia, no qual está sendo feita a análise. Observemos, ainda, que esta hipótese corresponde a hipótese do item 2, feita no enunciado do Teorema 1.1.3. Esta hipótese está relacionada, portanto, aos coeficientes da forma quadrática $Q$. Daqui em diante, neste capítulo, trabalharemos com esta hipótese. .

Observemos que, diante da Hipótese 8.2.1, os coeficientes $a$ e $b$ são não nulos. Assim diante dessa hipótese, vale a relação:

$$
\alpha b=\frac{a}{\alpha} \Longrightarrow a=\alpha^{2} b
$$

Portanto, substituindo na expressão de $Q=Q(x, y)$, conseguimos:

$$
Q(x, y)=\alpha^{2} b x^{2}+2 c x y-b y^{2}
$$

No entanto, estamos interessados na expressão da função $\mu_{1}$, na qual a função $Q$ aparece sendo calculada em valores $(x, y) \in \Gamma=H_{1}^{-1}\left(E_{1}\right)$. Logo, $(x, y)$ devem satisfazer:

$$
\frac{y^{2}}{2}+\frac{\alpha^{2} x^{2}}{2}+\frac{x^{4}}{2}=E_{1} \Longrightarrow y^{2}=2 E_{1}-\alpha^{2} x^{2}-x^{4}
$$

Substituindo esta relação na expressão de $Q$, obtemos:

$$
\begin{aligned}
& \left.Q\right|_{\Gamma}(x, y)=\alpha^{2} b x^{2}+2 c x y-b\left(2 E_{1}-\alpha^{2} x^{2}-x^{4}\right)= \\
& =\alpha^{2} b x^{2}+2 c x y-2 b E_{1}+b \alpha^{2} x^{2}+b x^{4}= \\
& =b x^{4}+2 b \alpha^{2} x^{2}+2 c x y-2 b E_{1} .
\end{aligned}
$$

Sendo $\mu_{1}(s)=Q(z(s))$, onde $z=(s)=(q(s), p(s)), q$ e $p$ dados em (8.5), obtemos então: 


$$
\mu_{1}(s)=b q^{4}(s)+2 \alpha^{2} b q^{2}(s)+2 c q(s) p(s)-2 b E_{1}
$$

Omitiremos o termo constante $2 b E_{1}$ dessa expressão (esse termo não interfere na análise). Escreveremos, para efeitos de simplificação nas expressões que serão manipuladas:

$$
\omega(s)=\left(\sqrt{r_{1}+r_{2}}\right) \cdot s
$$

Utilizando as expressões de $q$ e $p$, dadas em (8.5), obtemos:

$$
\mu_{1}(s)=b r_{1}^{2} c n^{4}(\omega(s))+2 \alpha^{2} b r_{1} c n^{2}(\omega(s))-c r_{1}\left(\sqrt{r_{1}+r_{2}}\right)(2 s n(\omega(s)) c n(\omega(s)) d n(\omega(s))) .
$$

Mas vale, entre as funções elípticas, a seguinte identidade, que está associada ao termo entre parênteses, na expressão acima:

$$
\operatorname{sn}(2 \omega(s))=\frac{2 \operatorname{sn}(\omega(s)) c n(\omega(s)) d n(\omega(s))}{1-k^{2} s n^{4}(\omega(s))} .
$$

Logo:

$$
\operatorname{sn}(2 \omega(s))\left(1-k^{2} s n^{4} \omega(s)\right)=2 \operatorname{sn}(\omega(s)) \operatorname{cn}(\omega(s)) d n(\omega(s)) .
$$

Substituindo na expressão de $\mu_{1}=\mu_{1}(s)$, obtemos:

$$
\begin{aligned}
& \mu_{1}(s)=b r_{1}^{2} c n^{4}(\omega(s))+2 \alpha^{2} b r_{1} c n^{2}(\omega(s))-c r_{1}\left(\sqrt{r_{1}+r_{2}}\right)(\operatorname{sn}(2 \omega(s)) \\
& \left(1-k^{2} s n^{4} \omega(s)\right)= \\
& =b r_{1}^{2} c n^{4}(\omega(s))+2 \alpha^{2} b r_{1} c n^{2}(\omega(s))-c r_{1}\left(\sqrt{r_{1}+r_{2}}\right) \operatorname{sn}(2 \omega(s))+ \\
& +c k^{2} r_{1}\left(\sqrt{r_{1}+r_{2}}\right) \operatorname{sn}(2 \omega(s)) s n^{4}(\omega(s)) .
\end{aligned}
$$

Portanto, obtemos a seguinte expressão para a função $\mu_{1}$ :

$$
\begin{aligned}
& \mu_{1}(s)=b r_{1}^{2} c n^{4}(\omega(s))+2 \alpha^{2} b r_{1} c n^{2}(\omega(s))-c r_{1}\left(\sqrt{r_{1}+r_{2}}\right) \operatorname{sn}(2 \omega(s))+ \\
& +c k^{2} r_{1}\left(\sqrt{r_{1}+r_{2}}\right) \operatorname{sn}(2 \omega(s)) s n^{4}(\omega(s)) .
\end{aligned}
$$

Como as funções $x \mapsto c n(x)$ e $x \mapsto s n(x)$ se estendem analiticamente a valores do plano complexo, a função $\mu_{1}$ se estenderá, também, a valores do plano complexo. Assim, teremos a função $\mu_{1}=\mu_{1}(z)$ :

$$
\mu_{1}(z)=b r_{1}^{2} c n^{4}(\omega(z))+2 \alpha^{2} b r_{1} c n^{2}(\omega(z))-c r_{1}\left(\sqrt{r_{1}+r_{2}}\right) \operatorname{sn}(2 \omega(z))+
$$




$$
+c k^{2} r_{1}\left(\sqrt{r_{1}+r_{2}}\right) \operatorname{sn}(2 \omega(z)) s n^{4}(\omega(z)) .
$$

As funções $z \mapsto c n(z)$ e $z \mapsto s n(z)$ são funções meromorfas duplamente periódicas. Seus períodos são, respectivamente, $4 K$ e $2 K+2 i K^{\prime}$ e $4 K$ e $2 i K^{\prime}$. Seguirá que as funções $z \mapsto c n(\omega(z))$ e $z \mapsto s n(\omega(z))$ possuem, respectivamente, períodos:

$$
\frac{4 K}{\sqrt{r_{1}+r_{2}}} \text { e } \frac{2 K+2 i K^{\prime}}{\sqrt{r_{1}+r_{2}}} \text { e } \frac{4 K}{\sqrt{r_{1}+r_{2}}} \text { e } \frac{2 i K^{\prime}}{\sqrt{r_{1}+r_{2}}} .
$$

Podemos notar que $4 K$ e $4 i K^{\prime}$ são períodos em comum das funções $c n$ e sn (são períodos em comum das funções $c n$, sn e $d n$; por isso, trabalharemos com eles). Portanto, a função $\mu_{1}$, estendida ao plano complexo, é uma função meromorfa, duplamente periódica, com períodos:

$$
\frac{4 K}{\sqrt{r_{1}+r_{2}}} \mathrm{e} \frac{4 i K^{\prime}}{\sqrt{r_{1}+r_{2}}}
$$

Em relação a essas constantes, temos a próxima observação.

Observação 8.2.1 Utilizando a forma da integral elíptica, apresentada nessa seção, teremos que a constante $K$ é dada por:

$$
\frac{K}{a^{2}+b^{2}}=\int_{0}^{b} \frac{d t}{\sqrt{\left(b^{2}-t^{2}\right)\left(a^{2}+t^{2}\right)}} .
$$

Assim, para o nosso caso, teremos:

$$
\frac{K}{r_{1}+r_{2}}=\int_{0}^{\sqrt{r_{1}}} \frac{d x}{\sqrt{\left(r_{1}-x^{2}\right)\left(r_{2}+x^{2}\right)}}
$$

Portanto, a constante $K$ está relacionada ao período da correspondente órbita do Sistema Hamiltoniano $H_{1}$. Teremos:

$$
\frac{4 K}{\sqrt{r_{1}+r_{2}}}=T_{1}
$$

A constante $K^{\prime}$ está relacionada ao módulo complementar $k^{\prime}$. Mais detalhes podem ser consultados em ([17]).

Resumindo, a função $\mu_{1}$, estendida ao plano complexo, é uma função meromorfa duplamente periódica. A próxima subseção se propõe a estudar as possíveis singularidades desta função. 


\subsection{As singularidades da função $\mu_{1}$}

Temos então:

$$
\begin{aligned}
& \mu_{1}(z)=b r_{1}^{2} c n^{4}(\omega(z))+2 \alpha^{2} b r_{1} c n^{2}(\omega(z))-c r_{1}\left(\sqrt{r_{1}+r_{2}}\right) \operatorname{sn}(2 \omega(z))+ \\
& +c k^{2} r_{1}\left(\sqrt{r_{1}+r_{2}}\right) \operatorname{sn}(2 \omega(z)) s n^{4}(\omega(z)) .
\end{aligned}
$$

Para fazer uma análise das possíveis singularidades da função $\mu_{1}$, definiremos as funções auxiliares:

- $A(z)=b r_{1}^{2} c n^{4}(\omega(z))+2 \alpha^{2} b r_{1} c n^{2}(\omega(z))$

- $B(z)=c k^{2} r_{1}\left(\sqrt{r_{1}+r_{2}}\right) \operatorname{sn}(2 \omega(z)) s n^{4}(\omega(z))$.

- $C(z)=c r_{1}\left(\sqrt{r_{1}+r_{2}}\right) \operatorname{sn}(2 \omega(z))$.

Dessa forma, teremos: $\mu_{1}(z)=A(z)+B(z)-C(z)$. Portanto, os candidatos às singularidades da função $\mu_{1}$ devem ser singularidades da função $A$, ou da função $B$, ou da função $C$. Iremos determinar as singularidades das funções $A, B$ e $C$ e, na medida do possível, os coeficientes de suas respectivas séries de Laurent, para, a partir disso, fazer o mesmo em relação a função $\mu_{1}$. Seguiremos por partes.

I- $\mathbf{A}$ função $\mathbf{A}=\mathbf{A}(\mathbf{z})$

Temos que a função $A$ é dada por:

$$
A(z)=b r_{1}^{2} c n^{4}(\omega(z))+2 \alpha^{2} b r_{1} c n^{2}(\omega(z)) .
$$

Seguirá que suas possíveis singularidades provém da função cosseno elíptico, $z \mapsto c n(z)$. Em relação às singularidades dessa função, temos:

Lema 8.3.1 Seja $y(z)=c n(z)$ a função cosseno elíptico, associado ao módulo $k$. Suas singularidades são $i K^{\prime}$ e $2 K+i K^{\prime}$ (ver a observação a seguir), que são polos simples, com resíduos dados, respectivamente, por $\frac{-i}{k} e \frac{i}{k}$.

Demonstração: $\operatorname{Ver}([17])$.

Em relação a esta afirmação, é importante destacar que a periodicidade dupla está sendo levada em consideração. Em relação a isso, temos a seguinte observação.

Observação 8.3.1 A situação deste lema pode ser analisada no paralelogramo com vértices nos seguintes pontos $-2 K, 2 K, 4 K+2 i K^{\prime}$ e $2 i K^{\prime}$. Todas as outras singularidades são equivalentes às singularidades descritas no lema, devido a periodicidade dupla. 
Sejam $z_{0}, z_{1} \in \mathbb{C}$ tais que $\omega\left(z_{0}\right)=i K^{\prime}$ e $\omega\left(z_{1}\right)=2 k+i K^{\prime}$. Assim, teremos:

$$
z_{0}=\frac{i K^{\prime}}{\sqrt{r_{1}+r_{2}}} \text { e } z_{1}=\frac{2 K+i K^{\prime}}{\sqrt{r_{1}+r_{2}}} .
$$

Segue, então, que $z_{0}$ e $z_{1}$ são singularidades da função $z \mapsto c n(\omega(z))$. A situação é resumida no próximo lema.

Lema 8.3.2 Os pontos:

$$
z_{0}=\frac{i K^{\prime}}{\sqrt{r_{1}+r_{2}}} e z_{1}=\frac{2 K+i K^{\prime}}{\sqrt{r_{1}+r_{2}}} .
$$

são polos de ordem 1 da função $z \mapsto c n(\omega(z))$. Os resíduos são, respectivamente, dados por: $\frac{-i}{k \sqrt{r_{1}+r_{2}}} e \frac{i}{k \sqrt{r_{1}+r_{2}}}$.

Observação 8.3.2 A situação é análoga à feita na observação referente ao lema anterior.

Diante dessas informações, levando-se em consideração as potências de $z \mapsto c n(\omega(z))$ e os coeficientes que estão na expressão da função $A=A(z)$, obtemos o próximo corolário.

Corolário 8.3.1 As singularidades da função $A=A(z)$ são:

$$
z_{0}=\frac{i K^{\prime}}{\sqrt{r_{1}+r_{2}}} \text { e } z_{1}=\frac{2 K+i K^{\prime}}{\sqrt{r_{1}+r_{2}}} .
$$

Temos ainda:

- $z_{0}$ é um polo de ordem 4 da função $A=A(z)$. A série de Laurent de A, centrada em $z_{0}$, é da forma:

$$
A(z)=\sum_{m=1}^{4} \frac{b_{m}}{\left(z-z_{0}\right)^{m}}+\sum_{n=0}^{\infty} a_{n}\left(z-z_{0}\right)^{n} .
$$

Com o coeficiente $b_{4}$, dado por:

$$
b_{4}=b r_{1}^{2}\left(\frac{-i}{k \sqrt{r_{1}+r_{2}}}\right)^{4}=b .
$$

- $z_{1}$ é um polo de ordem 4 da função $A=A(z)$. A sua série de Laurente, centrada em $z_{1}$, tem a forma de (8.6). Onde o coeficiente $b_{4}$ é dado por: 


$$
b_{4}=b r_{1}^{2}\left(\frac{i}{k \sqrt{r_{1}+r_{2}}}\right)^{4}=b
$$

Notemos que, devido ao módulo das funções elípticas ser dado em (8.3), teremos $b_{4}=b$. De fato:

$$
\begin{aligned}
& b_{4}=b r_{1}^{2}\left(\frac{ \pm i}{k \sqrt{r_{1}+r_{2}}}\right)^{4}=b r_{1}^{2}\left(\frac{ \pm i}{k \sqrt{r_{1}+r_{2}}}\right)^{2} \cdot\left(\frac{ \pm i}{k \sqrt{r_{1}+r_{2}}}\right)^{2}= \\
& =b r_{1}^{2}\left(\frac{-1}{k^{2}\left(r_{1}+r_{2}\right)}\right)\left(\frac{-1}{k^{2}\left(r_{1}+r_{2}\right)}\right)=b r_{1}^{2}\left(\frac{1}{\frac{r_{1}}{r_{1}+r_{2}}\left(r_{1}+r_{2}\right)}\right)\left(\frac{1}{\frac{r_{1}}{r_{1}+r_{2}}\left(r_{1}+r_{2}\right)}\right)= \\
& =b r_{1}^{2} \frac{1}{r_{1}^{2}}=b .
\end{aligned}
$$

O coeficiente $b$ é dado em (7.4). Temos a seguinte observação.

Observação 8.3.3 É importante notar que não há dependência entre coeficiente $b_{4}$ e a curva $\Gamma,(m, n)$-ressonante, que está sendo feita a análise. $O$ coeficiente $b$ depende do parâmetro $\alpha$ (na Hamiltoniana $H_{1}$ ) e da órbita periódica usada para a construção da aplicação de Poincaré. Posteriormente, voltaremos a destacar esta propriedades.

II- A Função $\mathrm{C}=\mathrm{C}(\mathrm{z})$.

A função $C=C(z)$ é dada por:

$$
C(z)=c r_{1}\left(\sqrt{r_{1}+r_{2}}\right) \operatorname{sn}(2 \omega(z)) .
$$

Segue que as possíveis singularidades da função $C$ estão relacionadas às singularidades da função $z \mapsto \operatorname{sn}(\omega(z))$. Em relação a isso, teremos:

Lema 8.3.3 Seja $y(z)=\operatorname{sn}(z)$, a função seno elíptico, associada ao módulo $k$. Suas singularidades são $i K^{\prime}$ e $2 K+i K^{\prime}$ (ver a observação a seguir), que são polos simples. Seus resíduos são dados, respectivamente, por $\frac{1}{k} e \frac{-1}{k}$.

É importante destacar "o fator "periodicidade, contido na afirmação desse lema, assim como fizemos com a função $z \mapsto c n(z)$.

Observação 8.3.4 A situação descrita no lema anterior pode ser analisada no paralelogramo com vértices $-K, 3 K, 3 K+2 i K^{\prime} e-K+2 i K^{\prime}$. Todas as outras singularidades serão equivalentes às singularidades descritas no lema, devido a periodicidade dupla. 
Sejam $z_{0}, z_{1} \in \mathbb{C}$, tais que $2 \omega\left(z_{0}\right)=i K^{\prime}$ e $2 \omega\left(z_{1}\right)=2 k+i K^{\prime}$. Então:

$$
z_{0}=\frac{i K^{\prime}}{2 \sqrt{r_{1}+r_{2}}} \text { e } z_{1}=\frac{2 K+i K^{\prime}}{2 \sqrt{r_{1}+r_{2}}}
$$

Seguirá que $z_{0}$ e $z_{1}$ são singularidades da função $z \mapsto s n(2 \omega(z))$. Outras informações são descritas no próximo lema.

Lema 8.3.4 Os pontos:

$$
z_{0}=\frac{i K^{\prime}}{2 \sqrt{r_{1}+r_{2}}} e z_{1}=\frac{2 K+i K^{\prime}}{2 \sqrt{r_{1}+r_{2}}}
$$

são singularidades ${ }^{2}$ da função $z \mapsto s n(2 \omega(z))$. Estas são polos de ordem 1 . Seus resíduos são dados, respectivamente, por:

$$
\frac{1}{2 k \sqrt{r_{1}+r_{2}}} e \frac{-1}{2 k \sqrt{r_{1}+r_{2}}} .
$$

Diante deste lema, podemos descrever as singularidades da função $C$.

Corolário 8.3.2 As singularidades da função $C=C(z)$ são dadas por:

$$
z_{0}=\frac{i K^{\prime}}{2 \sqrt{r_{1}+r_{2}}} e z_{1}=\frac{2 K+i K^{\prime}}{2 \sqrt{r_{1}+r_{2}}}
$$

Além disso, temos:

- $z_{0}$ é um polo de ordem 1 de $C$. O resíduo, associado, é dado por:

$$
b_{1}=c r_{1}\left(\sqrt{r_{1}+r_{2}}\right) \frac{1}{2 k \sqrt{r_{1}+r_{2}}}=\frac{c r_{1}}{2 k}
$$

- $z_{1}$ é polo de ordem 1 da função $C=C(z)$. O resíduo, associado, é dado por:

$$
b_{1}=-c r_{1}\left(\sqrt{r_{1}+r_{2}}\right) \frac{1}{2 k \sqrt{r_{1}+r_{2}}}=\frac{-c r_{1}}{2 k} .
$$

Com esse corolário, temos a descrição das singularidades da função $C$.

III- A função $\mathbf{B}=\mathbf{B}(\mathrm{z})$.

A função $B=B(z)$ é dada por:

\footnotetext{
${ }^{2}$ Devemos destacar que estas informações estão sempre condicionadas a periodicidade dupla
} 


$$
B(z)=c r_{1}\left(\sqrt{r_{1}+r_{2}}\right) k^{2} \operatorname{sn}(2 \omega(s)) s n^{4}(\omega(s)) .
$$

Observemos, então, que podemos escrever a função $B=B(z)$ da seguinte maneira:

$$
B(z)=C(z) \cdot\left(k^{2} s n^{4}(\omega(z))\right)
$$

Portanto, tanto as singularidades da função $C$, quanto as singularidades da função $z \mapsto k^{4} s n^{4}(\omega(z))$ serão candidatas a singularidades da função $B$. As singularidades de $C$ já foram estudadas. A seguir, caracterizaremos as singularidades da função $z \mapsto k^{2} s n^{4}(\omega(z))$.

Lema 8.3.5 Os pontos:

$$
z_{0}=\frac{i K^{\prime}}{\sqrt{r_{1}+r_{2}}} \text { e } z_{1}=\frac{2 K+i K^{\prime}}{\sqrt{r_{1}+r_{2}}} .
$$

são singularidades ${ }^{3}$ da função $z \mapsto k^{2} s n^{4}(\omega(z))$. Estes são polos de ordem 4. Os coeficientes da correspondentes série de Laurent são, respectivamente, dados por:

$$
\begin{aligned}
& \text { 1. } k^{2}\left(\frac{1}{k}\right)^{4}=k^{2} \frac{1}{k^{4}}=\frac{1}{k^{2}} ; \\
& \text { 2. } k^{2}\left(\frac{-1}{k}\right)^{4}=k^{2} \frac{1}{k^{4}}=\frac{1}{k^{2}} \text {. }
\end{aligned}
$$

Diante desse lema, teremos os seguintes candidatos a singularidades da função $B$ :

- Singularidades de $C=C(z)$ são $\frac{i K^{\prime}}{2 \sqrt{r_{1}+r_{2}}}$ e $\frac{2 K+i K^{\prime}}{2 \sqrt{r_{1}+r_{2}}}$ são polos de ordem 1, com coeficientes, respectivamente, dados por: $\frac{c r_{1}}{2 k}$ e $\frac{c r_{1}}{2 k}$, conforme discutido anteriormente.

- Singularidades de $z \mapsto k^{2} s n^{4}(\omega(z))$ são $\frac{i K^{\prime}}{\sqrt{r_{1}+r_{2}}}$ e $\frac{2 K+i K^{\prime}}{\sqrt{r_{1}+r_{2}}}$ são polos de ordem 4, com, respectivamente, coeficientes: $\frac{1}{k^{2}} \mathrm{e} \frac{1}{k^{2}}$.

Observemos que, calculando a função $z \mapsto k^{2} s n^{4}(\omega(z))$ nas singularidades da função $C$, obtemos:

\footnotetext{
${ }^{3}$ Devemos sempre considerar essa afirmação, a menos de periodicidade dupla.
} 


$$
k^{2} s n^{4}\left(\omega\left(\frac{i K^{\prime}}{2 \sqrt{r_{1}+r_{2}}}\right)\right)=k^{2} s n^{4}\left(\frac{i K^{\prime}}{2}\right)=k^{2}\left(\frac{i}{\sqrt{k}}\right)^{4}=k^{2} \frac{1}{k^{2}}=1 .
$$

Da mesma forma, teremos que:

$$
k^{2} s n^{4}\left(\omega\left(\frac{2 K+i K^{\prime}}{2 \sqrt{r_{1}+r_{2}}}\right)\right)=k^{2} \operatorname{sn}^{4}\left(\frac{2 K+i K^{\prime}}{2}\right)=k^{2} s n^{4}\left(K+\frac{i K^{\prime}}{2}\right) .
$$

Devemos notar que vale a seguinte relação entre funções elípticas (ver $([17]))$ :

$$
\operatorname{sn}\left(K+\frac{i K^{\prime}}{2}\right)=\operatorname{sn}\left(\frac{-i K^{\prime}}{2}+\left(K+i K^{\prime}\right)\right)=\frac{1}{k} \frac{d n\left(\frac{-i K^{\prime}}{2}\right)}{\operatorname{cn}\left(\frac{-i K^{\prime}}{2}\right)}=\frac{1}{k} \frac{\sqrt{1+k}}{\frac{\sqrt{1+k}}{\sqrt{k}}}=\frac{1}{\sqrt{k}} .
$$

Logo:

$$
k^{2} s n^{4}\left(\omega\left(\frac{2 K+i K^{\prime}}{2 \sqrt{r_{1}+r_{2}}}\right)\right)=k^{2}\left(\frac{1}{\sqrt{k}}\right)^{4}=1 .
$$

Resumindo, calculando a função $z \mapsto k^{2} s n^{4}(\omega(z))$ nas singularidades da função $C$, obtemos:

$$
k^{2} \operatorname{sn}^{4}\left(\omega\left(\frac{i K^{\prime}}{2 \sqrt{r_{1}+r_{2}}}\right)\right)=1 \text { e } k^{2} s n^{4}\left(\omega\left(\frac{2 K+i K^{\prime}}{2 \sqrt{r_{1}+r_{2}}}\right)\right)=1
$$

Portanto, a análise das singularidades da função $B$, em relação às singularidades da função $C$, pode ser feita na situação do próximo lema.

Lema 8.3.6 Sejam $^{4} f: A\left(z_{0}, 0, \delta\right) \rightarrow \mathbb{C}$ e $g: B_{\delta}\left(z_{0}\right) \rightarrow \mathbb{C}$ funções holomorfas. Definimos $h: A\left(z_{0}, 0, \delta\right) \rightarrow \mathbb{C}$ por $h(z)=f(z) g(z)$. Se $z_{0}$ é um polo de ordem 1 de $f$, com resíduo $\zeta$, e $g\left(z_{0}\right) \neq 0$, então $z_{0}$ é um polo de ordem 1 da função $h$, com resíduo $\zeta \cdot g\left(z_{0}\right)$.

Demonstração: Como $z_{0}$ é polo de ordem 1 da função $f$, com resíduo $\zeta$, teremos:

$$
\lim _{z \rightarrow z_{0}}\left(z-z_{0}\right) f(z)=\zeta
$$

Observemos que:

$$
\left(z-z_{0}\right) h(z)=\left(z-z_{0}\right) f(z) g(z)=\left\{\left(z-z_{0}\right) f(z)\right\} g(z) .
$$

Segue então:

$$
\lim _{z \rightarrow z_{0}}\left(z-z_{0}\right) h(z)=\zeta \cdot g\left(z_{0}\right) \neq 0
$$

\footnotetext{
${ }^{4}$ Notações usuais de análise complexa. Ver [32].
} 
Isso conclui a afirmação do lema.

Este lema nos permite caracterizar as singularidades de $C$ como singularidades da função $B$.

Corolário 8.3.3 Em relação a função $B=B(z)$, vale::

- Os pontos $z_{0}=\frac{i K^{\prime}}{2 \sqrt{r_{1}+r_{2}}}$ e $z_{1}=\frac{2 K+i K^{\prime}}{2 \sqrt{r_{1}+r_{2}}}$ são singularidades de $B$;

- $z_{0}$ é um polo de ordem 1 , com resíduo $\frac{c r_{1}}{2 k}$;

- $z_{1}$ é um polo de ordem 1 , com resíduo $\frac{c r_{1}}{2 k}$.

Observação 8.3.5 Notemos que estes são, exatamente, os polos da função $C$ (Ou seja, os polos são os mesmos e os resíduos são os mesmos.)

Devemos avaliar, agora, as possibilidades em relação as singularidades da função $z \mapsto k^{2} s n^{4}(\omega(z))$. Calculando a função $C$ nas singularidades da função $z \mapsto k^{2} s n^{4}(\omega(z))$, obtemos:

$$
\begin{aligned}
& C\left(\frac{i K^{\prime}}{\sqrt{r_{1}+r_{2}}}\right)=c r_{1}\left(\sqrt{r_{1}+r_{2}}\right) \operatorname{sn}\left(2\left(\sqrt{r_{1}+r_{2}}\right) \frac{i K^{\prime}}{r_{1}+r_{2}}\right)=c r_{1}\left(r_{1}+r_{2}\right) \operatorname{sn}\left(2 i K^{\prime}\right) \\
& =c r_{1}\left(\sqrt{r_{1}+r_{2}}\right) \operatorname{sn}\left(0+2 K^{\prime} i\right)=0
\end{aligned}
$$

Fazendo o mesmo em relação a outra singularidade:

$$
\begin{aligned}
& C\left(\frac{2 K+2 i K^{\prime}}{\sqrt{r_{1}+r_{2}}}\right)=c r_{1}\left(\sqrt{r_{1}+r_{2}}\right) \operatorname{sn}\left(2\left(\sqrt{r_{1}+r_{2}}\right) \frac{2 K+2 i K^{\prime}}{\sqrt{r_{1}+r_{2}}}\right)= \\
& =c r_{1}\left(\sqrt{r_{1}+r_{2}}\right) \operatorname{sn}\left(4 K+2 i K^{\prime}\right)=0 .
\end{aligned}
$$

Portanto, o argumento, que foi usado na situação anterior, não pode ser utilizado aqui. Uma observação fundamental, que pode ser encontrada em ([17]), é que os zeros das funções elípticas de Jacobi são todos simples. Em relação a essa segunda situação, em que as singularidades de uma função são zeros da outra, temos:

Lema 8.3.7 Suponhamos $f: A\left(z_{0}, 0, \delta\right) \rightarrow \mathbb{C}$ e $g: B_{\delta}\left(z_{0}\right) \rightarrow \mathbb{C}$ holomorfas, sendo $z_{0}$ um polo de ordem 4 de $f$ e $z_{0}$ um zero simples da função $g$. Definimos $h: A\left(z_{0}, 0, \delta\right) \rightarrow \mathbb{C}$, por $h(z)=f(z) g(z)$. Então $z_{0}$ é um polo de ordem 3 da função $h$.

Demonstração: Como $z_{0}$ é polo de ordem 4 de $f, \exists \lambda \in \mathbb{C} \backslash\{0\}$ tal que: 


$$
\lim _{z \rightarrow z_{0}}\left(z-z_{0}\right)^{4} f(z)=\lambda
$$

Sendo $z_{0}$ um zero de ordem 1 de g, podemos escrever:

$$
g(z)=\left(z-z_{0}\right) g_{1}(z) \text {, com } g_{1}\left(z_{0}\right) \neq 0 .
$$

Segue, então, que:

$$
\begin{aligned}
& \left(z-z_{0}\right)^{3} h(z)=\left(z-z_{0}\right)^{3} f(z) g(z)=\left(z-z_{0}\right)^{3} f(z)\left(z-z_{0}\right) g_{1}(z) \\
& =\left(z-z_{0}\right)^{4} f(z) g_{1}(z) .
\end{aligned}
$$

Logo:

$$
\lim _{z \rightarrow z_{0}}\left(z-z_{0}\right)^{3} h(z)=\lim _{z \rightarrow z_{0}}\left(z-z_{0}\right)^{4} f(z) g_{1}(z)=\lambda g_{1}\left(z_{0}\right) \neq 0
$$

Segue que $z_{0}$ é polo de ordem 3 de $h$.

Corolário 8.3.4 Os pontos $z_{0}=\frac{i K^{\prime}}{\sqrt{r_{1}+r_{2}}}$ e $z_{1}=\frac{2 K+i K^{\prime}}{\sqrt{r_{1}+r_{2}}}$ são polos de ordem 3 da função $B=B(z)$.

Observação 8.3.6 Devemos notar que estes mesmos pontos são também polos de ordem 4 da função $A=A(z)$.

A situação, referente a "todas" as singularidades da função $B=B(z)$ é resumida no próximo corolário.

Corolário 8.3.5 A função $B=B(z)$ possui as seguintes singularidades:

- $z_{1}=\frac{i K^{\prime}}{2 \sqrt{r_{1}+r_{2}}}$ e $z_{2}=\frac{2 K+i K^{\prime}}{2 \sqrt{r_{1}+r_{2}}}$.

Estes são polos de ordem 1, com residuos, respectivamente, dados por $\frac{c r_{1}}{2 k} e \frac{c r_{1}}{2 k}$.

- $z_{3}=\frac{i K^{\prime}}{\sqrt{r_{1}+r_{2}}}$ e $z_{4}=\frac{2 K+i K^{\prime}}{\sqrt{r_{1}+r_{2}}}$.

Estes são polos de ordem 3 de $B$.

Com este último corolário, concluímos a análise das singularidades de $B$. Iremos voltar a análise da função $\mu_{1}=\mu_{1}(z)$. 
IV-Sobre as singularidades da função $\mu_{1}$.

A função $\mu_{1}$ é dada por $\mu_{1}(z)=A(z)+B(z)-C(z)$. Diante da análise feita, os candidatos a singularidades da função $\mu_{1}$ são singularidades das funções $A, B$ e $C$, que são descritas nos corolários 8.3.1, 8.3.5 e 8.3.2. Portanto, serão os pontos $z_{1}, z_{2}, z_{3}$ e $z_{4}$, dados no corolário 8.3.5. Observemos que $z_{1}$ e $z_{2}$ são singularidades das funções $B$ e $C$. Ambas são polos de ordem 1 e possuem os mesmos resíduos. Ao fazermos " $B-C$ " na expressão de $\mu_{1}$, estas singularidades são removidas. Ou seja, elas são singularidades removíveis da função $\mu_{1}$. Por outro lado, os pontos $z_{3}$ e $z_{4}$ são polos das funções $A$ e $B$. No entanto, são polos de ordem 4 da função $A$ e polos de ordem 3 da função $B$. Logo, os pontos $z_{3}$ e $z_{4}$ são polos de ordem 4 da função $\mu_{1}$. Essas informações estão na próxima proposição.

Proposição 8.3.1 A função $\mu_{1}$ possui as seguintes singularidades:

$$
z_{1}=\frac{i K^{\prime}}{\sqrt{r_{1}+r_{2}}} \text { e } z_{2}=\frac{2 K+i K^{\prime}}{\sqrt{r_{1}+r_{2}}}
$$

Além disso, temos:

- $z_{1}$ é polo de ordem 4 da função $\mu_{1}$. O coeficiente associado, na série de Laurent de $\mu_{1}$, centrada em $z_{1}$, é $b_{4}=b$;

- $z_{2}$ é polo de ordem 4 da função $\mu_{1}$. O coeficiente associado, na série de Laurent de $\mu_{1}$, centrada em $z_{2}$, é $b_{4}=b$;

A seguir analisaremos "a contribuição" de $\mu_{1}$ para o somatório, relacionado a expressão de Melnikov.

\subsection{A Contribuição de $\mu_{1}$ ao Somatório.}

A soma que estamos interessados em analisar é dada por:

$$
L(s)=\sum_{j=0}^{n-1} \mu\left(s+j \cdot T_{2}\right) .
$$

Essa expressão depende inteiramente da curva $(m, n)$-ressonante que estamos analisando. Se essa expressão não for identicamente nula, então a curva em questão não persiste sob a perturbação do sistema (conforme estabelecido na definição (6.1.1). A função $\mu$ é da forma $\mu=\mu_{1}+\mu_{2}$. Consideremos a parcela da soma de $L$, relacionada a função $\mu_{1}$. Ou seja, definimos $L^{1}: \mathbb{R} \rightarrow \mathbb{R}$ dada por: 


$$
L^{1}(s)=\sum_{j=0}^{n-1} \mu_{1}\left(s+j \cdot T_{2}\right) .
$$

Nesta subseção, vamos mostrar como as informações da proposição 8.3.1, a respeito da função $\mu_{1}$, nos permite concluir que a função $L^{1}$ não é constante ${ }^{5}$; em particular, $L^{1}$ não é identicamente nula.

Primeiramente, analisemos a periodicidade da função $L^{1}$.

Lema 8.4.1 A função $L^{1}: \mathbb{R} \rightarrow \mathbb{R}$ possui períodos $T_{2}$ e $T_{1}=\frac{4 K}{\sqrt{r_{1}+r_{2}}}$.

Demonstração: A função $s \mapsto \mu_{1}(s)$ está associada a orbita periódica do sistema hamiltoniano $X_{H_{1}}$, que tem período $T_{1}$. Portanto, $T_{1}$ é um período de $L^{1}$. Analisemos a situação referente a $T_{2}$.

Observemos que:

$$
\frac{T_{2}}{T_{1}}=\frac{m}{n} \Longrightarrow n T_{2}=m T_{1}
$$

Diante disso, teremos:

$$
\mu_{1}\left(s+n T_{2}\right)=\mu_{1}\left(s+m T_{1}\right)=\mu_{1}(s) .
$$

Portanto, na expressão de $L^{1}=L^{1}(s)$, conseguimos:

$$
\begin{aligned}
& L^{1}\left(s+T_{2}\right)=\sum_{j=0}^{n-1} \mu_{1}\left(s+j T_{2}+T_{2}\right)=\sum_{j=0}^{n-1} \mu_{1}\left(s+(j+1) T_{2}\right)= \\
& =\sum_{j=1}^{n} \mu_{1}\left(s+j T_{2}\right)=\sum_{j=1}^{n-1} \mu_{1}\left(s+j T_{2}\right)+\left(\mu_{1}\left(s+n T_{2}\right)\right)= \\
& =\sum_{j=0}^{n-1} \mu_{1}\left(s+j T_{2}\right)=L^{1}(s) .
\end{aligned}
$$

Isso mostra que $T_{2}$ também é um período da função $L^{1}$. Isso conclui o lema.

Sendo $T_{1}$ e $T_{2}$ períodos de $L^{1}$, temos que qualquer combinação linear entre eles, com coeficientes em $\mathbb{Z}$, também será um período. Iremos focar na combinação que nos fornece o período $T_{1}=\frac{4 K}{\sqrt{r_{1}+r_{2}}}$.

Como a função $\mu_{1}$ se estende analiticamente, ao plano complexo, a uma função meromorfa, e como se trata de uma soma finita, a função $L^{1}$ também se estende analiticamente a uma função meromorfa. Obtemos assim uma função:

\footnotetext{
${ }^{5}$ Sendo assim, restaria analisar a interferência do termo $\mu_{2}$ nessa situação.
} 


$$
L^{1}(z)=\sum_{j=0}^{n-1} \mu_{1}\left(z+j \cdot T_{2}\right) .
$$

Segue, das propriedades de $\mu_{1}$ e do lema anterior, que essa extensão é duplamente periódica, com períodos:

$$
\frac{4 K}{\sqrt{r_{1}+r_{2}}} \text { e } \frac{4 K^{\prime} i}{\sqrt{r_{1}+r_{2}}} .
$$

Portanto, a extensão de $L^{1}=L^{1}(z)$ é uma função elíptica. Analisemos possíveis singularidades para essa extensão $L^{1}=L^{1}(z)$. Candidatos naturais seriam os pontos $z_{1}$ e $z_{2}$, descritos na proposição 8.3.1, por se tratarem de singularidades de da função $\mu_{1}=\mu_{1}(z)$. O próximo lema irá nos dizer se o ponto $z_{1}$ é singularidade do $j$-ésimo termo da soma, $z \mapsto \mu_{1}\left(z+j T_{2}\right)$. Essas expressões estão relacionadas a curva $(m, n)$-ressonante.

Lema 8.4.2 Se $n$ é impar, então $z_{1}$ é singularidade de $z \mapsto \mu_{1}\left(z+j T_{2}\right)$ apenas se $j=0$. Se $n$ é par, então $z_{1}$ é singularidade de $z \mapsto \mu_{1}\left(z+j T_{2}\right)$ para $j=0$ e $j=\frac{n}{2}$.

Demonstração: Utilizando a proposição 8.3.1, teremos que $z_{1}$ será singularidade de $z \mapsto \mu_{1}\left(z+j T_{2}\right)$ nas seguintes situações:

$$
\begin{aligned}
& \text { 1. } z_{1}+j \cdot T_{2} \in z_{1}+\frac{4 K}{\sqrt{r_{1}+r_{2}}} \mathbb{Z}+\frac{4 K^{\prime} i}{\sqrt{r_{1}+r_{2}}} \mathbb{Z} ; \\
& \text { 2. } z_{1}+j \cdot T_{2} \in z_{2}+\frac{4 K}{\sqrt{r_{1}+r_{2}}} \mathbb{Z}+\frac{4 K^{\prime} i}{\sqrt{r_{1}+r_{2}}} \mathbb{Z}
\end{aligned}
$$

Analisemos separadamente cada uma dessas situações.

1. Observemos que essa situação ocorre se, e somente se, a seguinte condição é satisfeita:

$$
j T_{2} \in \frac{4 K}{\sqrt{r_{1}+r_{2}}} \mathbb{Z}
$$

Mas como $T_{2}=\frac{m}{n} \frac{4 K}{\sqrt{r_{1}+r_{2}}}$, teremos:

$$
j T_{2} \in \frac{4 K}{\sqrt{r_{1}+r_{2}}} \mathbb{Z} \Leftrightarrow j m \in n \mathbb{Z} .
$$

Como $0 \leq j<n$ e $m$ e $n$ são primos entre si, essa equivalência ocorre apenas se $j=0$. Observemos que essa análise independe de $n$ ser par ou impar. 
2. Essa condição ocorre se, e somente se, a seguinte condição é satisfeita:

$$
j T_{2} \in \frac{1}{2} \frac{4 K}{\sqrt{r_{1}+r_{2}}}+\frac{4 K}{\sqrt{r_{1}+r_{2}}} \mathbb{Z} .
$$

Uma vez que vale a seguinte relação:

$$
z_{2}-z_{1}=\frac{2 K}{\sqrt{r_{1}+r_{2}}}=\frac{1}{2} \frac{4 K}{\sqrt{r_{1}+r_{2}}} .
$$

Utilizando que $T_{2}=\frac{m}{n} \frac{4 K}{\sqrt{r_{1}+r_{2}}}$, obtemos que a condição acima é equivalente a:

$$
j \frac{m}{n} \in \frac{1}{2}+\mathbb{Z}
$$

Observemos que essa condição não é satisfeita para $j=0$. Caso ela seja satisfeita, deverá existir $p_{j} \in \mathbb{Z}$, tal que:

$$
j \frac{m}{n}=\frac{1}{2}+p_{j}=\frac{1+2 p_{j}}{2} \Longrightarrow 2 j m=n\left(1+2 p_{j}\right) .
$$

Mas sendo $m$ e $n$ primos entre si, tem de existir $\lambda_{j} \in \mathbb{N}$ tal que $2 j=$ $\lambda_{j} n$. Observemos que:

$$
j<n \Longrightarrow \lambda_{j} n=2 j<2 n \therefore \lambda_{j}<2 \text {. }
$$

Assim, $\lambda_{j}=0$ ou $\lambda_{j}=1$. O primeiro caso não nos interessa. Logo, $\lambda_{j}=1$, ou seja, $j=\frac{n}{2}$. Essa condição, evidentemente, ocorre apenas se $n$ for um número par.

Concluímos, assim, as afirmações do lema.

A próxima proposição mostrará que a função elíptica $L^{1}=L^{1}(z)$ possui $z_{1}$ como uma singularidade.

Proposição 8.4.1 A extensão analítica de $L^{1}(z)=\sum_{j=0}^{n-1} \mu_{1}\left(z+j T_{2}\right)$ possui um polo de ordem $4 \mathrm{em} z_{1}$. Além disso, se $n$ é par, então o coeficiente na série de Laurent associado é dado por $2 b$. Se $n$ é ímpar, então este coeficiente é $b$ (onde b é dado por (7.4)).

Demonstração: Faremos, separadamente, essa análise nos casos $n$ par e $n$ impar. 
- $n$ impar;

Se $n$ é ímpar, então, de acordo com o lema anterior, $z_{1}$ será singularidade de $z \mapsto \mu_{1}\left(z+j T_{2}\right)$ apenas para $j=0$. Logo:

$$
L^{1}\left(z_{1}+u\right)=\sum_{j=0}^{n-1} \mu_{1}\left(z_{1}+u+j T_{2}\right)=\mu_{1}\left(z_{1}+u\right)+\sum_{j=1}^{n-1} \mu_{1}\left(z_{1}+u+j T_{2}\right) .
$$

Utilizando a proposição (8.3.1) e o lema anterior, obtemos:

$$
L^{1}\left(z_{1}+u\right)=\frac{b}{u^{4}}+\frac{b_{3}}{u^{3}}+\frac{b_{2}}{u^{2}}+\frac{b_{1}}{u}+\ldots
$$

Como $b \neq 0$, segue que $z_{1}$ é um polo de ordem 4 da função $L^{1}=L^{1}(z)$.

- $n$ par;

Para o caso $n$ par, $z_{1}$ será singularidade de $z \mapsto \mu_{1}\left(z+j T_{2}\right)$ nos casos $j=0$ e $j=\frac{n}{2}$. Logo:

$$
\begin{aligned}
& L^{1}\left(z_{1}+u\right)=\sum_{j=0}^{n-1} \mu_{1}\left(z_{1}+u+j T_{2}\right)=\mu_{1}\left(z_{1}+u\right)+\mu_{1}\left(z_{1}+u+\frac{n}{2} T_{2}\right)+ \\
& \sum_{j=1, j \neq \frac{n}{2}}^{n-1} \mu_{1}\left(z_{1}+u+j T_{2}\right) .
\end{aligned}
$$

Pelo lema anterior, existem $p_{1}, p_{2} \in \mathbb{Z}$ tal que:

$$
z_{1}+\frac{n}{2} T_{2}=z_{2}+\frac{4 K p_{1}}{\sqrt{r_{1}+r_{2}}}+\frac{4 p_{2} K^{\prime} i}{\sqrt{r_{1}+r_{2}}}
$$

Assim:

$$
\mu_{1}\left(z_{1}+u+\frac{n}{2} T_{2}\right)=\mu_{1}\left(z_{2}+\frac{4 K p_{1}}{\sqrt{r_{1}+r_{2}}}+\frac{4 p_{2} K^{\prime} i}{\sqrt{r_{1}+r_{2}}}+u\right)=\mu_{1}\left(z_{2}+u\right),
$$

onde a última igualdade se deve a periodicidade da função $\mu_{1}$. Obtemos, através da soma anterior, então:

$$
L^{1}\left(z_{1}+u\right)=\mu_{1}\left(z_{1}+u\right)+\mu_{1}\left(z_{2}+u\right)+\sum_{j=1, j \neq \frac{n}{2}}^{n-1} \mu_{1}\left(z_{1}+u+j T_{2}\right) .
$$

Utilizando a proposição (8.3.1) e o lema anterior, obtemos: 


$$
L^{1}\left(z_{1}+u\right)=\frac{b}{u^{4}}+\frac{b}{u^{4}}+\sum_{j=1}^{3} \frac{c_{j}}{u^{j}}+\ldots
$$

Portanto:

$$
L^{1}\left(z_{1}+u\right)=\frac{2 b}{u^{4}}+\sum_{j=1}^{3} \frac{c_{j}}{u^{j}}+\ldots
$$

Como $b \neq 0$, segue que $z_{1}$ é polo de ordem 4 de $L^{1}=L^{1}(z)$.

Segue o resultado.

Com essas informações, obtemos o seguinte corolário.

Corolário 8.4.1 A função $L^{1}: \mathbb{R} \rightarrow \mathbb{R}$ não é uma função constante. Em particular, não é identicamente nula.

Demonstração: Segue do fato de que sua extensão analítica ao plano complexo é uma função elíptica com um polo de ordem 4, conforme descrito no lema anterior.

Diante disso, pela construção que vem sendo feita, é conveniente colocar o resultado do corolário anterior em termos das coordenadas $(x, y) \in \Omega$ da seção.

Corolário 8.4.2 Existe uma órbita $\left(x_{i}, y_{i}\right)_{0 \leq i \leq n-1}$ de $\psi_{0}$, sobre a curva $(m, n)$ ressonante, $\Gamma$, tal que:

$$
\sum_{i=0}^{n-1} Q\left(x_{i}, y_{i}\right) \neq 0
$$

Além disso, podemos supor, sem perda de generalidade, que $Q\left(x_{i}, y_{i}\right) \neq 0, \forall i$.

$\mathrm{Na}$ sequência do texto, mostraremos como o último corolário e as informações a respeito das funções ${ }^{6} Q=Q(x, y)$ e $R=R(x, y)$ nos permitem concluir que a função $L=L(s)$ não é identicamente nula.

${ }^{6}$ Ver a observação 7.5.2. 


\subsection{Conclusão a respeito da destruição de cur- vas invariantes}

Pela a análise que vem sendo feita, para concluir que a curva $\Gamma$ não persiste sob a perturbação, que estamos analisando, é suficiente mostrar que:

$$
\text { Existe uma órbita }\left(x_{j}, y_{j}\right) \text { de } \psi_{0} \text {, tal que } \sum_{j=0}^{n-1} A_{1}\left(x_{j}, y_{j}\right) \neq 0 \text {. }
$$

Sendo $A_{1}(x, y)=Q(x, y)+R(x, y)$, o corolário 8.4 .2 nos fornece uma órbita candidata a verificar esta desigualdade. Fixaremos, então, tal órbita $\left(x_{i}, y_{i}\right)_{0 \leq i \leq n-1}$. A análise é baseada na observação 7.5.2. Em relação a forma quadrática $Q$, temos uma outra observação importante.

Observação 8.5.1 A forma quadrática $Q$ possui zeros. No que segue, como os pontos da órbita fixada não são zeros de $Q$, na análise que faremos, podemos supor que $Q$ não se anula. Assim, se $Z \subseteq \mathbb{R}^{2}$ representa os pontos que anula $Q$, trabalharemos no aberto $\mathbb{R}^{2} \backslash Z$.

Lema 8.5.1 Vale o seguinte limite ${ }^{7}$ :

$$
\lim _{(x, y) \rightarrow(0,0)} \frac{|R(x, y)|}{|Q(x, y)|}=0
$$

Demonstração: Observemos que:

$$
\frac{|R(x, y)|}{\|(x, y)\|^{2}}=\frac{|R(x, y)|}{|Q(x, y)|} \frac{|Q(x, y)|}{\|(x, y)\|^{2}} .
$$

Ao fazermos $(x, y) \rightarrow(0,0)$, na igualdade acima, o termo da esquerda vai a zero e o termo $\frac{|Q(x, y)|}{\|(x, y)\|^{2}}$ é limitado. Segue que $\frac{|R(x, y)|}{|Q(x, y)|}$ tende a zero, quando $(x, y) \rightarrow(0,0)$.

Com as informações deste lema, temos o seguinte resultado.

Lema 8.5.2 Dado $\epsilon_{1} \in(0,1)$, se a curva $\Gamma$ estiver suficientemente próxima de $(0,0)$, então vale a seguinte estimativa para a soma da função $A_{1}$ sobre a órbita $\left(x_{i}, y_{i}\right)_{0 \leq i \leq n-1}$, fixada acima:

$$
\left(1-\epsilon_{1}\right) \sum_{i=0}^{n-1} Q\left(x_{i}, y_{i}\right)<\sum_{i=0}^{n-1} A_{1}\left(x_{i}, y_{i}\right)<\left(1+\epsilon_{1}\right) \sum_{i=0}^{n-1} Q\left(x_{i}, y_{i}\right) .
$$

\footnotetext{
${ }^{7}$ Tendo em vista a observação anterior.
} 


\subsection{CONCLUSÃO A RESPEITO DA DESTRUIÇÃO DE CURVAS INVARIANTES117}

Demonstração: Pelo lema anterior, dado $\epsilon_{1} \in(0,1)$, existe $\delta>0$, tal que:

$$
\|(x, y)\|<\delta \Longrightarrow-\epsilon_{1}|Q(x, y)|<R(x, y)<\epsilon_{1}|Q(x, y)| .
$$

Podemos assumir que a curva $(m, n)$-ressonante está contida na bola $B_{\delta}(0,0)$. Portanto, esta desigualdade valerá sobre os pontos da órbita $\left(x_{i}, y_{i}\right)_{0 \leq i \leq n-1}$. Mas como $A_{1}(x, y)=Q(x, y)+R(x, y)$, teremos:

$\sum_{i} A_{1}\left(x_{i}, y_{i}\right)=\sum_{i}\left\{Q\left(x_{i}, y_{i}\right)+R\left(x_{i}, y_{i}\right)\right\}>\sum_{i}\left\{Q\left(x_{i}, y_{i}\right)-\epsilon_{1}\left|Q\left(x_{i}, y_{i}\right)\right|\right\}$.

O termo geral do somatório do lado direito desta desigualdade pode ser analisado separadamente, segundo o sinal de $\left|Q\left(x_{i}, y_{i}\right)\right|$. Indicaremos índice $j$ para o caso de $Q$ ser positivo e $k$ para ocaso de $Q$ ser negativo. Logo:

$$
\begin{aligned}
& \sum_{i} A_{1}\left(x_{i}, y_{i}\right)>\sum_{j}\left\{Q\left(x_{j}, y_{j}\right)-\epsilon_{1} Q\left(x_{j}, y_{j}\right)\right\}+\sum_{k}\left\{Q\left(x_{k}, y_{k}\right)+\epsilon_{1} Q\left(x_{k}, y_{k}\right)\right\}= \\
& =\left(1-\epsilon_{1}\right) \sum_{j} Q\left(x_{j}, y_{j}\right)+\left(1+\epsilon_{1}\right) \sum_{k} Q\left(x_{k}, y_{k}\right)> \\
& >\left(1-\epsilon_{1}\right) \sum_{j} Q\left(x_{j}, y_{j}\right)+\left(1-\epsilon_{1}\right) \sum_{k} Q\left(x_{k}, y_{k}\right)= \\
& =\left(1-\epsilon_{1}\right) \sum_{i=0}^{n-1} Q\left(x_{i}, y_{i}\right) .
\end{aligned}
$$

Desta forma, conseguimos uma das desigualdades afirmadas no lema. O processo de obtenção da outra desigualdade é, inteiramente, análogo. Segue $o$ resultado.

Por meio desse último lema, obtemos o seguinte resultado fundamental.

Proposição 8.5.1 Em relação a órbita $\left(x_{i}, y_{i}\right)_{0 \leq i \leq n-1}$, temos:

$$
\sum_{i=0}^{n-1} A_{1}\left(x_{i}, y_{i}\right) \neq 0 .
$$

Portanto, a curva $\Gamma$, que é $(m, n)$-ressonante, não persiste sob a perturbação. Demonstração: Segue do corolário 8.4.2 e do lema anterior.

${ }^{8}$ Ver a observação 7.1.1. 
Diante desse corolário, obtemos:

Demonstração do Teorema 1.1.3: A partir do último corolário, temos que para toda curva $\Gamma$, que é $(m, n)$-ressonante e está suficientemente próxima de $(0,0) \in \Omega_{0}$, existe uma órbita $\left(x_{i}, y_{i}\right)_{0 \leqslant i \leqslant n-1}$ da aplicação $\psi_{0}$, em $\Gamma$, tal que:

$$
\sum_{i=0}^{n-1} A_{1}\left(x_{i}, y_{i}\right) \neq 0
$$

Segue da Proposição da discussão feita no Capítulo 7, que a curva $\Gamma$ não persiste sob a perturbação $\psi_{\epsilon}=\psi_{0}+o(\epsilon)$. A não persistência dessas curvas $(m, n)$-ressonantes, arbitrariamente próximas ao ponto fixo $(0,0) \in$ $\Omega_{0}$, fazem surgir regiões de instabilidade, para $\psi_{\epsilon}, \epsilon$ pequeno, se acumulando em $(0,0) \in \Omega_{\epsilon}$. Analisando-se esta situação ao longo da órbita periódica $\Gamma_{E}$, concluímos a demonstração do Teorema 1.1.3.

Em relação ao Corolário 1.1.1, temos:

Demonstração do Corolário 1.1.1: Quando uma curva $(m, n)$-ressoante é destruída, surge uma região anular invariante por $\psi_{\epsilon}$, sem curvas invariantes em seu interior, conjugada a uma aplicação twist, para $\epsilon$ suficientemente pequeno (ou seja, regiões de instabilidade). A demonstração deste corolário segue do resultado de [1]. Veja a Observação 2.4.3.

Na próxima seção, iremos discutir a dependência desses resultados em relação ao nível de energia.

\subsection{Algumas Observações Finais}

Diante da última seção, concluímos as demonstrações dos resultados enunciados no Capítulo 1. É importante destacar que as hipóteses feitas nos Teoremas 1.1.1, 1.1.2 e 1.1.3 são hipóteses sobre níveis de energia do sistema hamiltoniano definido em (1.1). De fato, para cada nível $E>0$, no Lema 1.1.1, fixamos uma órbita periódica $\Gamma_{E}$ de $X_{H_{\epsilon}}$, para todo $\epsilon$, com energia $E$ e período $T=T(E)$. Se $\alpha \cdot T(E)$ não for múltiplo inteiro de $2 \pi$, então, para $|\epsilon|$ pequeno, $\Gamma_{E}$ é uma órbita periódica de $X_{H_{\epsilon}}$, que é elíptica em seu nível de energia.

Excluindo-se mais alguns níveis de energia (isso é feito impondo-se $E \in$ $J)$, conseguimos que a Forma Normal de Birkhoff, para esta órbita $\Gamma_{E}$, esteja definida, para $|\epsilon|$ pequeno, e, dessa forma, o primeiro invariante de Birkhoff, para o caso $\epsilon=0$, pode ser visto como uma função da energia. Portanto, temos definida a função $I_{B}: J \rightarrow \mathbb{R}$, que associa a cada nível $E$, o primeiro invariante de Birkhoff, para o caso $\epsilon=0, I_{B}(E)$. Se $I_{B}(E) \neq 0$, então o primeiro invariante de $\Gamma_{E}$, órbita de $X_{H_{\epsilon}}$, é não nulo, para $|\epsilon|$ pequeno. Em 
consequência, obtemos toros invariantes pelo fluxo do campo $X_{H_{\epsilon}}$, com energia $E$, que se acumulam em $\Gamma_{E}$. Esse é o conteúdo do Teorema 1.1.2. Pela Proposição 5.3.1, temos que $\lim _{E \rightarrow 0} I_{B}(E)=-\infty$, devido a influência da singularidade sela-centro, localizada no nível zero de energia. Com isso, obtemos "muitos níveis de energia "próximos ao nível zero, satisfazendo as hipóteses dos Teoremas 1.1.1 e 1.1.2.

A hipótese 2 do teorema 1.1.3 também pode ser vista como uma condição sobre o nível de energia $E \in J$. De fato, podemos escrevê-la como:

$$
\int_{0}^{T(E)} x_{2}^{2}(s, E) \sin \{2 \alpha(T(E)-s)\} d s \neq 0 .
$$

A partir dela, mostramos a existência de zonas de instabilidade, que se acumulam na órbita elíptica $\Gamma_{E}$ de $X_{H_{\epsilon}}$, para $|\epsilon|$ pequeno. Portanto, em relação ao sistema hamiltoniano definido em (1.1), temos que os resultados apresentados dependem de condições sobre o nível de energia e que existem níveis de energia, próximos ao nível zero, que satisfazem nossas condições.

A existência da órbita periódica hiperbólica $\Gamma_{E}^{\prime}$ nos remete a uma situação interessante, ainda a ser explorada, descrita em nos trabalhos [12], [13] e [11]. De fato, em [13], mostra-se um resultado de entropia topológica positiva, para sistemas hamiltonianos no $\mathbb{R}^{4}$, com uma singularidade sela-centro, no nível zero de energia, satisfazendo uma condição de convexidade neste nível de energia (ver [30] para uma definição de convexidade). A partir de um sistema de folheação transversal (ver [11] e [12] para uma definição) e por meio da análise próxima a uma órbita hiperbólica, que, no sistema definido em (1.1), coincide com a órbita $\Gamma_{E}^{\prime}$, mostra-se o resultado de entropia topológica positiva. Portanto, se estabelecermos um resultado de convexidade para o sistema (1.1), válido para $|\epsilon|$ pequeno, poderíamos obter uma figura com as duas órbitas, $\Gamma_{E}$ e $\Gamma_{E}^{\prime}$ linkadas, com o comportamento próximo a $\Gamma_{E}^{\prime}$ descrito em [13], e o comportamento próximo a órbita elíptica $\Gamma_{E}$, descrito neste texto. Há um método para demonstrar esta condição de convexidade. Tal método, assim como as definições e propriedades envolvidas, são descritas em [30] (ver também [15]).

Uma outra situação possível, a ser explorada, consiste numa descrição mais detalhada da dinâmica das aplicações de Poincaré nas regiões de instabilidade, dadas pelo Teorema 1.1.3. De fato, o comportamento genérico de difeomorfismos analíticos do plano, que preservam área, tendo um ponto fixo elíptico, foi estudado por Zehnder, em [34] (ver também [35]). Poderíamos nos questionar se as zonas de instabilidade, descritas no Teorema 1.1.3, se comportam como na situação genérica de Zehnder ou o quanto se aproximam dessa situação genérica. Trata-se de uma situação natural a ser explorada. 


\section{Apêndice A}

\section{As derivadas do campo $f_{1}$.}

Neste apêndice, trataremos de alguns cálculos envolvendo as derivadas do fluxo do campo $X_{H_{\epsilon}}$. Estamos admitindo o contexto do início da seção 8. O objetivo é demonstrar as igualdades da proposição 7.6.1. Alguns fatos sobre propriedades de fluxos de equações diferenciais ordinárias, assim como propriedades da derivada do fluxo em relação a parâmetros e condições iniciais podem ser consultados em [33].

\section{A.1 Reescrevendo as Transformações de Poin- caré}

Temos, conforme foi descrito no texto, a família de transformações de Poincaré, nas coordenadas da seção, $\psi_{\epsilon}: \Omega \rightarrow \Omega$, com $f_{\epsilon}=\psi_{\epsilon} \circ \psi_{0}^{-1}$. Vimos, também, que as derivadas de ordem 2 do termo de Melnikov, descritas nas coordenadas da seção (a função $A_{1}$ ), podem ser escritas em termos das derivadas de $f_{1}=\left.\frac{\partial}{\partial \epsilon}\right|_{\epsilon=0} f_{\epsilon}$ (proposição 7.5.2.) Reescreveremos as transformações $\psi_{\epsilon}$ de maneira conveniente, de modo que possamos relacionar as derivadas do campo $f_{1}$ com o fluxo Hamiltoniano do campo $X_{H_{\epsilon}}$. Para isso, vamos reescrever as transformações de Poincaré em termos dos correspondentes fluxos.

Analisaremos as transformações como aplicações em $\mathbb{R}^{4}$ e, em seguida, passaremos para as coordenadas da seção. Consideremos:

- $T_{\epsilon}=T+R_{\epsilon}$; onde $T_{\epsilon}$ é a aplicação tempo de retorno para o sistema $X_{H_{\epsilon}}, T$ é o período da órbita periódica. Assim ${ }^{1}, R_{\epsilon}$ é a aplicação que nos dá a diferença entre o tempo de retorno e o período da órbita.

\footnotetext{
${ }^{1}$ Por abuso de notação, usaremos, apenas neste apêndice, $R_{\epsilon}$ para representar a aplicação que nos dá a diferença do tempo de retorno em relação ao período.
} 
- $T_{0}=T+R_{0}$; onde $T_{0}$ é a aplicação tempo de retorno para o sistema $X_{H_{0}}, T$ é o período da órbita e $R_{0}$ é a aplicação que indica a diferença entre o tempo de retorno e o período da órbita periódica.

- Essas funções estão definidas sobre a seção e são diferenciáveis.

Se $z \in \Sigma_{E}^{+}$, então teremos as aplicações de Poincaré:

$$
\varphi_{\epsilon}(z)=\phi^{H_{\epsilon}}\left(T_{\epsilon}(z), z\right) \text { e } \varphi_{0}(z)=\phi^{H_{0}}\left(T_{0}(z), z\right) .
$$

Assim, $\varphi_{\epsilon}$ e $\varphi_{0}$ representam $\psi_{\epsilon}$ e $\psi_{0}$ escritas em termos dos seus respectivos fluxos Hamiltonianos no $\mathbb{R}^{4}$.

Faremos expansões do fluxo, em relação a varável tempo e em relação a variável posição. Assim, seguiremos por partes.

1. Em relação a variável $t$, em $t=T$.

$$
\phi^{H_{\epsilon}}(t+T, z)=\phi^{H_{\epsilon}}(T, z)+t X_{H_{\epsilon}}\left(\phi^{H_{\epsilon}}(z)\right)+r_{\epsilon, 1}(t, z) .
$$

Fazendo $t=R_{\epsilon}(z)$, obtemos:

$$
\varphi_{\epsilon}(z)=\phi^{H_{\epsilon}}(T, z)+R_{\epsilon}(z) X_{H_{\epsilon}}\left(\phi_{T}^{H_{\epsilon}}(z)\right)+r_{\epsilon, 1}(z) .
$$

Procedendo da mesma forma para o caso $\epsilon=0$, conseguimos:

$$
\varphi_{0}(z)=\phi^{H_{0}}(T, z)+R_{0}(z) X_{H_{0}}\left(\phi_{T}^{H_{0}}(z)\right)+r_{0,1}(z) .
$$

Nessas expressões, faremos as expansões dos termos $\phi_{T}^{H_{\epsilon}}, \phi_{T}^{H_{0}}, X_{H_{\epsilon}} \circ \phi_{T}^{H_{\epsilon}}$ e $X_{H_{0}} \circ \phi_{T}^{H_{0}}$ em $z=z_{0}$, onde $z_{0}$ é o ponto periódico.

2. $\phi_{T}^{H_{\epsilon}}(z)$ e $\phi_{T}^{H_{0}}(z)$

Teremos:

$$
\phi_{T}^{H_{\epsilon}}(z)=\phi_{T}^{H_{\epsilon}}\left(z_{0}\right)+d_{z_{0}} \phi_{\bar{T}}^{H_{\epsilon}}\left(z-z_{0}\right)+\tilde{r}_{1, \epsilon}(z) .
$$

Da mesma forma para $\epsilon=0$ :

$$
\phi_{T}^{H_{0}}(z)=\phi_{T}^{H_{0}}\left(z_{0}\right)+d_{z_{0}} \phi_{\bar{T}}^{H_{0}}\left(z-z_{0}\right)+\tilde{r}_{1,0}(z) .
$$


3. $X_{H_{\epsilon}} \circ \phi_{T}^{H_{\epsilon}}(z)$ e $X_{H_{0}} \circ \phi_{T}^{H_{0}}(z)$

Teremos:

$$
\begin{aligned}
& X_{H_{\epsilon}}\left(\phi_{T}^{H_{\epsilon}}(z)\right)=X_{H_{\epsilon}}\left(\phi_{T}^{H_{\epsilon}}\left(z_{0}\right)\right)+d_{z_{0}}\left(X_{H_{\epsilon}} \circ \phi_{T}^{H_{\epsilon}}\right)\left(z-z_{0}\right)+s_{1, \epsilon}(z)= \\
& =X_{H_{0}}\left(\phi_{T}^{H_{\epsilon}}\left(z_{0}\right)\right)+d_{z_{0}}\left(X_{H_{\epsilon}} \circ \phi_{T}^{H_{\epsilon}}\right)\left(z-z_{0}\right)+s_{1, \epsilon}(z) .
\end{aligned}
$$

Procedendo de maneira análoga para o caso $\epsilon=0$, obtemos:

$$
X_{H_{0}}\left(\phi_{T}^{H_{0}}(z)\right)=X_{H_{0}}\left(\phi_{T}^{H_{0}}\left(z_{0}\right)\right)+d_{z_{0}}\left(X_{H_{0}} \circ \phi_{T}^{H_{0}}\right)\left(z-z_{0}\right)+s_{1,0}(z) .
$$

Essas expressões nos permitem escrever:

$$
\begin{aligned}
& \varphi_{\epsilon}(z)=z_{0}+d_{z_{0}} \phi_{T}^{H_{\epsilon}}\left(z-z_{0}\right)+ \\
& +R_{\epsilon}(z)\left\{X_{H_{0}}\left(z_{0}\right)+d_{z_{0}} X_{H_{0}}\left(d_{z_{0}} \phi_{T}^{H_{\epsilon}}\left(z-z_{0}\right)\right)\right\}+Q_{\epsilon}(z) .
\end{aligned}
$$

e

$$
\begin{aligned}
& \varphi_{0}(z)=z_{0}+d_{z_{0}} \phi_{T}^{H_{0}}\left(z-z_{0}\right)+ \\
& +R_{0}(z)\left\{X_{H_{0}}\left(z_{0}\right)+d_{z_{0}} X_{H_{0}}\left(d_{z_{0}} \phi_{T}^{H_{0}}\left(z-z_{0}\right)\right)\right\}+Q_{0}(z) .
\end{aligned}
$$

Onde:

$$
\left\{\begin{array}{l}
Q_{\epsilon}(z)=\tilde{r}_{1, \epsilon}(z)+r_{1, \epsilon}(z)+R_{\epsilon}(z) s_{1, \epsilon}(z), \\
Q_{0}(z)=\tilde{r}_{1,0}(z)+r_{1,0}(z)+R_{0}(z) s_{1,0}(z) .
\end{array}\right.
$$

Portanto, conseguimos:

$$
\begin{aligned}
& \varphi_{\epsilon}(z)-\varphi_{0}(z)=\left(d_{z_{0}} \phi_{T}^{H_{\epsilon}}-d_{z_{0}} \phi_{T}^{H_{0}}\right)\left(z-z_{0}\right)+\left(R_{\epsilon}(z)-R_{0}(z)\right) X_{H_{0}}\left(z_{0}\right)+ \\
& +R_{\epsilon}(z) d_{z_{0}} X_{H_{0}}\left(d_{z_{0}} \phi_{T}^{H_{\epsilon}}\left(z-z_{0}\right)\right)-R_{0}(z) d_{z_{0}} X_{H_{0}}\left(d_{z_{0}} \phi_{T}^{H_{0}}\left(z-z_{0}\right)\right)+ \\
& \left.+Q_{\epsilon}(z)-Q_{0}(z) \doteq G_{\epsilon}(z) \text { (definição de } G_{\epsilon}\right) .
\end{aligned}
$$

Segue que:

$$
\varphi_{\epsilon}(z)=\varphi_{0}(z)+G_{\epsilon}(z) .
$$


Vamos, agora, relacionar esta igualdade com as expressões das transformações de Poincaré, quando escritas no sistema de coordenadas $(x, y) \in \Omega$ da seção. Observemos que a seção pode ser dada por:

$$
\begin{gathered}
\bar{z}: \Omega \rightarrow \mathbb{R}^{4} \\
(x, y) \mapsto(x, 0, y, \bar{y}(x, y)),
\end{gathered}
$$

onde $\bar{y}$ é dado pela igualdade (3.5).

Teremos em coordenadas:

$$
\psi_{\epsilon}=\Pi \circ \varphi_{\epsilon} \circ \bar{z}
$$

onde $\Pi: \mathbb{R}^{4} \rightarrow \mathbb{R}$, é a projeção $\Pi\left(x_{1}, x_{2}, y_{1}, y_{2}\right)=\left(x_{1}, y_{1}\right)$. Segue que:

$$
\begin{aligned}
& \psi_{\epsilon}(x, y)=\Pi\left(\varphi_{\epsilon}(\bar{z}(x, y))\right)=\Pi\left(\varphi_{0}(\bar{z}(x, y))+\Pi\left(G_{\epsilon}(\bar{z}(x, y))\right)=\right. \\
& =\psi_{0}(x, y)+\Pi\left(G_{\epsilon}(\bar{z}(x, y))\right) .
\end{aligned}
$$

Baseado nessa igualdade, definimos $\bar{G}_{\epsilon}(x, y)=\Pi\left(G_{\epsilon}(\bar{z}(x, y))\right)$. Assim:

$$
\psi_{\epsilon}(x, y)=\psi_{0}(x, y)+\bar{G}_{\epsilon}(x, y) .
$$

O objetivo, conforme foi observado, seria relacionar as derivadas de $f_{1}$ com as propriedades do campo $X_{H_{\epsilon}}$. Observemos que quando escrevemos $\psi_{\epsilon}(x, y)=\psi_{0}(x, y)+\bar{G}_{\epsilon}(x, y)$, as propriedades de $X_{H_{\epsilon}}$ aparecem explicitamente no termo $\bar{G}_{\epsilon}$, através de $G_{\epsilon}=G_{\epsilon}(z)$. A próxima proposição relaciona $d_{(0,0)} f_{1} \operatorname{com} \bar{G}_{\epsilon}$.

Proposição A.1.1 Vale a seguinte igualdade:

$$
d f_{1}(0,0)=d \bar{G}_{1}(0,0) \circ d \psi_{0}^{-1}(0,0)
$$

Demonstração: Sendo $\psi_{\epsilon}=f_{\epsilon} \circ \psi_{0}$, derivando esta igualdade com respeito a variável $\epsilon$ e calculando $e m \epsilon=0$, obtemos:

$$
\psi_{1}=f_{1} \circ \psi_{0} .
$$

Por outro lado, temos que $\psi_{\epsilon}=\psi_{0}+\bar{G}_{\epsilon}$. Derivando com respeito a variável $\epsilon$ e calculando em $\epsilon=0$, obtemos:

$$
\psi_{1}=\bar{G}_{1} .
$$

Igualando essas duas igualdades, obtemos: $\bar{G}_{1}=f_{1} \circ \psi_{0}$. Derivando essa igualdade e calculando em $(x, y)=(0,0)$, conseguimos: $d f_{1}(0,0)=$ $d \bar{G}_{1}(0,0) \circ d \psi_{0}^{-1}(0,0)$. Segue o resultado. 
Gostaríamos de calcular $d_{(0,0)} f_{1}$ por meio desta igualdade. O cálculo de $\left(d_{(0,0)} \psi_{0}\right)^{-1}$ é, relativamente, simples. Vamos analisar melhor a expressão de $\bar{G}_{\epsilon}$, visando o cálculo de $d_{(0,0)} \bar{G}_{1}$.

Teremos:

$$
\bar{G}_{\epsilon}(x, y)=\Pi\left(G_{\epsilon}(\bar{z}(x, y))\right) .
$$

Sendo que:

$$
\begin{aligned}
& G_{\epsilon}(z)=\left(d_{z_{0}} \phi_{T}^{H_{\epsilon}}-d_{z_{0}} \phi_{T}^{H_{0}}\right)\left(z-z_{0}\right)+\left(R_{\epsilon}(z)-R_{0}(z)\right) X_{H_{0}}\left(z_{0}\right)+ \\
& +R_{\epsilon}(z) d_{z_{0}} X_{H_{0}}\left(d_{z_{0}} \phi_{T}^{H_{\epsilon}}\left(z-z_{0}\right)\right)-R_{0}(z) d_{z_{0}} X_{H_{0}}\left(d_{z_{0}} \phi_{T}^{H_{0}}\left(z-z_{0}\right)\right)+ \\
& +Q_{\epsilon}(z)-Q_{0}(z) .
\end{aligned}
$$

Nessa igualdade, objetivando cálculos que virão a frente, indicaremos as transformações lineares, que aparecem nessa igualdade, por suas respectivas representações matriciais. Indicaremos $d_{z_{0}} \phi_{T}^{H_{\epsilon}}=\Phi_{\epsilon}(T)$ e $C=d_{z_{0}} X_{H_{0}}$. Tendo em vista que a aplicação П, que é uma projeção, também está em sua forma matricial. Assim, teremos:

$$
\bar{G}_{\epsilon}(x, y)=\Pi\left(\Phi_{\epsilon}(T)-\Phi_{0}(T)\right)\left(\bar{z}(x, y)-z_{0}\right)+R_{\epsilon}(\bar{z}(x, y)) \Pi C \Phi_{\epsilon}(T)(\bar{z}(x, y)-
$$
$\left.z_{0}\right)-R_{0}(\bar{z}(x, y)) \Pi C \Phi_{0}(T)\left(\bar{z}(x, y)-z_{0}\right)+\Pi\left(Q_{\epsilon}(\bar{z}(x, y))-Q_{0}(\bar{z}(x, y))\right.$.

Derivando com respeito a $\epsilon$, obtemos:

$$
\begin{aligned}
& \bar{G}_{1}(x, y)=\Pi \Phi_{1}(T)\left(\bar{z}(x, y)-z_{0}\right)+R_{1}(\bar{z}(x, y)) \Pi C \Phi_{0}(T)\left(\bar{z}(x, y)-z_{0}\right)+ \\
& +R_{0}(\bar{z}(x, y)) \Pi C \Phi_{1}(T)\left(\bar{z}(x, y)-z_{0}\right)+\Pi\left(Q_{1}(\bar{z}(x, y))\right) . \\
& \text { O próximo lema irá analisar o último termo: }
\end{aligned}
$$

Lema A.1.1 Vale a igualdade:

$$
d_{(0,0)}\left(\Pi \circ Q_{1} \circ \bar{z}\right)=0 .
$$

Demonstração: Segue da definição da função $Q_{1}$.

Como nosso objetivo é calcular $d \bar{G}_{1}(0,0)$, desprezaremos o último termo $\Pi\left(Q_{1}(\bar{z}(x, y))\right)$, uma vez que esse termo, pelo lema anterior, não irá contribuir com $d_{(0,0)} \bar{G}_{1}$. Portanto:

$$
\bar{G}_{1}(x, y)=\Pi \Phi_{1}(T)\left(\bar{z}(x, y)-z_{0}\right)+R_{1}(\bar{z}(x, y)) \Pi C \Phi_{0}(T)\left(\bar{z}(x, y)-z_{0}\right)+
$$


$+R_{0}(\bar{z}(x, y)) \Pi C \Phi_{1}(T)\left(\bar{z}(x, y)-z_{0}\right)$

Nessa expressão, conforme foi mencionado, temos as matrizes $\Phi_{\epsilon}(T)$, que consiste na representação matricial de $d_{z_{0}} \phi_{t}^{H_{\epsilon}}$, em $t=T$. A matriz $C$ consiste na representação de $d_{z_{0}} X_{H_{0}}$. Devemos notar que $d_{z_{0}} X_{H_{0}}$ depende apenas de coeficientes da Hamiltoniana $H_{0}$. Assim, num certo sentido, no nosso contexto, é uma matriz constante. De fato, como $X_{H_{0}}\left(x_{1}, x_{2}, y_{1}, y_{2}\right)=$ $\left(y_{1}, y_{2},-V_{1}^{\prime}\left(x_{1}\right),-V_{2}^{\prime}\left(x_{2}\right)\right)$ e $z_{0}=\left(0,0,0, y_{2}^{E}\right)$, temos:

$$
d_{z_{0}} X_{H_{0}}=\left[\begin{array}{cccc}
0 & 0 & 1 & 0 \\
0 & 0 & 0 & 1 \\
-\alpha^{2} & 0 & 0 & 0 \\
0 & -a_{2} & 0 & 0
\end{array}\right]=C
$$

A projeção $\Pi: \mathbb{R}^{4} \longrightarrow \mathbb{R}^{2}, \Pi\left(x_{1}, x_{2}, y_{1}, y_{2}\right)=\left(x_{1}, y_{1}\right)$ é indicada, em notação matricial, pela matriz $\Pi$. Assim:

$$
\Pi=\left[\begin{array}{llll}
1 & 0 & 0 & 0 \\
0 & 0 & 1 & 0
\end{array}\right]
$$

Vamos avaliar a matriz $\Phi_{\epsilon}(T)$.

\section{A.2 As Matrizes $\Phi_{\epsilon}(t)$}

A transformação linear $d_{z_{0}} \phi_{\bar{T}}^{H_{\epsilon}}$ é a solução da seguinte equação diferencial matricial:

$$
\left\{\begin{array}{l}
X^{\prime}=A_{\epsilon}(t) X, \\
X(0)=I d .
\end{array}\right.
$$

Onde $A_{\epsilon}(t)=d X_{H_{\epsilon}}\left(\phi_{t}^{H_{\epsilon}}\left(z_{0}\right)\right)$. Indicando a órbita periódica de $z_{0}$ por $\left(0, x_{2}(t), 0, y_{2}(t)\right)$, teremos então:

$$
A_{\epsilon}(t)=\left[\begin{array}{cccc}
0 & 0 & 1 & 0 \\
0 & 0 & 0 & 1 \\
-\alpha^{2}-2 \epsilon x_{2}^{2}(t) & 0 & 0 & 0 \\
0 & -a_{2}-3 b_{2} x_{2}^{2}(t) & 0 & 0
\end{array}\right] .
$$

Estamos indicando a solução da equação (A.2) acima por $\Phi_{\epsilon}(t)$. Assim, $\Phi_{\epsilon}(T)=d_{z_{0}} \phi_{T}^{H_{\epsilon}}$.

Temos que as entradas de $A_{\epsilon}(t)$ que dependem de $\epsilon$ e $t$ serão indicadas por:

$$
\left\{\begin{array}{l}
u_{\epsilon}(t)=-\alpha^{2}-2 \epsilon x_{2}^{2}(t), \\
v(t)=-a_{2}-3 b_{2} x_{2}^{2}(t) .
\end{array}\right.
$$


Com isso, podemos reescrever a matriz $A_{\epsilon}(t)$ de forma simplificada:

$$
A_{\epsilon}(t)=\left[\begin{array}{cccc}
0 & 0 & 1 & 0 \\
0 & 0 & 0 & 1 \\
u_{\epsilon}(t) & 0 & 0 & 0 \\
0 & v(t) & 0 & 0
\end{array}\right]
$$

Representaremos esta matriz por blocos de matrizes $2 \times 2$. Logo:

$$
A_{\epsilon}(t)=\left[\begin{array}{cc}
\mathbb{O} & \mathbb{I} \\
U_{\epsilon}(t) & \mathbb{O}
\end{array}\right]
$$

Indiquemos, também, a matriz $\Phi_{\epsilon}(t)$, solução de (A.2), por blocos de matrizes $2 \times 2$. Logo:

$$
\Phi_{\epsilon}(t)=\left[\begin{array}{cc}
X & Y \\
Z & W
\end{array}\right]
$$

Omitindo a dependência das variáveis $\epsilon$ e $t$ nos blocos $2 \times 2$. Como $\Phi_{\epsilon}(t)$ é solução de (A.2), teremos:

$$
\begin{gathered}
{\left[\begin{array}{cc}
X^{\prime} & Y^{\prime} \\
Z^{\prime} & W^{\prime}
\end{array}\right]=\Phi_{\epsilon}^{\prime}(t)=A_{\epsilon}(t) \Phi_{\epsilon}(t)=\left[\begin{array}{cc}
\mathbb{O} & \mathbb{I} \\
U_{\epsilon}(t) & \mathbb{O}
\end{array}\right]\left[\begin{array}{cc}
X & Y \\
Z & W
\end{array}\right]} \\
=\left[\begin{array}{cc}
Z & W \\
U_{\epsilon}(t) X & U_{\epsilon}(t) Y
\end{array}\right] .
\end{gathered}
$$

Portanto, tendo em vista essas igualdades e as condições iniciais, o sistema se torna:

- $X^{\prime}=Z, X(0)=\mathbb{I}$.

- $Y^{\prime}=W, Y(0)=\mathbb{O}$.

- $Z^{\prime}=U_{\epsilon}(t) X, Z(0)=\mathbb{O}$.

- $W^{\prime}=U_{\epsilon}(t) Y, W(0)=\mathbb{I}$.

Essas equações se reduzem às seguintes equações diferenciais matriciais de ordem 2:

$$
\left\{\begin{array}{l}
X^{\prime \prime}=U_{\epsilon}(t) X \\
X(0)=\mathbb{I} \\
X^{\prime}(0)=\mathbb{O}
\end{array}\right.
$$


Da mesma forma em $Y$ :

$$
\left\{\begin{array}{l}
Y^{\prime \prime}=U_{\epsilon}(t) Y \\
Y(0)=\mathbb{O} \\
Y^{\prime}(0)=\mathbb{I}
\end{array}\right.
$$

Analisemos, separadamente, cada uma das equações (A.4) e (A.5).

1. Equação (A.4)

Vamos supor que a solução da equação (A.4) seja da forma:

$$
X(t)=\left[\begin{array}{ll}
x(t) & y(t) \\
z(t) & w(t)
\end{array}\right]
$$

Teremos, então:

$$
\begin{aligned}
{\left[\begin{array}{ll}
x^{\prime \prime} & y^{\prime \prime} \\
z^{\prime \prime} & z^{\prime \prime}
\end{array}\right]=X^{\prime \prime}=U_{\epsilon}(t) X } & =\left[\begin{array}{cc}
u_{\epsilon}(t) & 0 \\
0 & v(t)
\end{array}\right]\left[\begin{array}{cc}
x & y \\
z & w
\end{array}\right]= \\
& =\left[\begin{array}{cc}
u_{\epsilon}(t) x & u_{\epsilon}(t) y \\
v(t) z & v(t) w
\end{array}\right] .
\end{aligned}
$$

Por esta igualdade e pelas condições iniciais, podemos reduzir às equações unidimensionais de segunda ordem:

- $x^{\prime \prime}=u_{\epsilon}(t) x, x(0)=1$ e $x^{\prime}(0)=0$;

- $y^{\prime \prime}=u_{\epsilon}(t) y, y(0)=0$ e $y^{\prime}(0)=0 \Longrightarrow y \equiv 0$.

- $z^{\prime \prime}=v(t) z, z(0)=0$ e $z^{\prime}(0)=0 \Longrightarrow z \equiv 0$.

- $w^{\prime \prime}=v(t) w, w(0)=1$ e $w^{\prime}(0)=0$.

Concluímos, então, que a matriz, $2 \times 2, X=X(t)$ é da forma:

$$
X(t)=\left[\begin{array}{cc}
x_{1}(t) & 0 \\
0 & w_{1}(t)
\end{array}\right]
$$

Onde:

$$
\left\{\begin{array}{l}
x_{1}^{\prime \prime}=u_{\epsilon}(t) x_{1} \\
x_{1}(0)=1 \\
x_{1}^{\prime}(0)=0
\end{array}\right.
$$


E, da mesma forma para $w_{1}=w_{1}(t)$ :

$$
\left\{\begin{array}{l}
w_{1}^{\prime \prime}=v(t) w_{1} \\
w_{1}(0)=1 \\
w_{1}^{\prime}(0)=0
\end{array}\right.
$$

Passemos para a equação (A.5)

2. Equação (A.5)

Utilizando a mesma ideia para a equação (A.5), escreveremos:

$$
Y(t)=\left[\begin{array}{cc}
x & y \\
z & w
\end{array}\right]
$$

Segue que a matriz, $2 \times 2, Y=Y(t)$ satisfaz:

$$
\begin{aligned}
{\left[\begin{array}{ll}
x^{\prime \prime} & y^{\prime \prime} \\
z^{\prime \prime} & z^{\prime \prime}
\end{array}\right]=Y^{\prime \prime}=U_{\epsilon}(y) Y } & =\left[\begin{array}{cc}
u_{\epsilon}(t) & 0 \\
0 & v(t)
\end{array}\right]\left[\begin{array}{cc}
x & y \\
z & w
\end{array}\right]= \\
& =\left[\begin{array}{cc}
u_{\epsilon}(t) x & u_{\epsilon}(t) y \\
v(t) z & v(t) w
\end{array}\right] .
\end{aligned}
$$

Dessa igualdade e das condições iniciais, reduzimos às seguintes equações unidimensionais de segunda ordem:

- $x^{\prime \prime}=u_{\epsilon}(t) x, x(0)=0$ e $x^{\prime}(0)=1$;

- $y^{\prime \prime}=u_{\epsilon}(t) y, y(0)=0$ e $y^{\prime}(0)=0 \Longrightarrow y \equiv 0$.

- $z^{\prime \prime}=v(t) z, z(0)=0$ e $z^{\prime}(0)=0 \Longrightarrow z \equiv 0$.

- $w^{\prime \prime}=v(t) w, w(0)=0$ e $w^{\prime}(0)=1$.

Logo, a matriz, $2 \times 2, Y=Y(t)$ é da forma:

$$
X(t)=\left[\begin{array}{cc}
x_{2}(t) & 0 \\
0 & w_{2}(t)
\end{array}\right] .
$$


Onde:

$$
\left\{\begin{array}{l}
x_{2}^{\prime \prime}=u_{\epsilon}(t) x_{2} \\
x_{2}(0)=0 \\
x_{2}^{\prime}(0)=1
\end{array}\right.
$$

E, da mesma forma para $w=w(t)$ :

$$
\left\{\begin{array}{l}
w_{2}^{\prime \prime}=v(t) w_{2} \\
w_{2}(0)=0 \\
w_{2}^{\prime}(0)=1
\end{array}\right.
$$

Segue dessa análise das matrizes, $2 \times 2, X$ e $Y$, e tendo em vista que os blocos da matriz $\Phi_{\epsilon}(t)$ satisfazem: $X^{\prime}=W$ e $Y^{\prime}=W$, obtemos que:

$$
\Phi_{\epsilon}(t)=\left[\begin{array}{cc}
X & Y \\
X^{\prime} & Y^{\prime}
\end{array}\right]=\left[\begin{array}{cccc}
x_{1}(t) & 0 & x_{2}(t) & 0 \\
0 & w_{1}(t) & 0 & w_{2}(t) \\
x_{1}^{\prime}(t) & 0 & x_{2}^{\prime}(t) & 0 \\
0 & w_{1}^{\prime}(t) & 0 & w_{2}^{\prime}(t)
\end{array}\right] .
$$

Calculando essa matriz em $t=T$, obtemos $\Phi_{\epsilon}(T)$ :

$$
\Phi_{\epsilon}(\bar{T})=\left[\begin{array}{cccc}
a_{11} & 0 & b_{11} & 0 \\
0 & a_{22} & 0 & b_{22} \\
c_{11} & 0 & d_{11} & 0 \\
0 & c_{22} & 0 & d_{22}
\end{array}\right]
$$

Nesse ponto, é fundamental notar que as soluções das equações (A.7) e (A.9) não dependem do parâmetro $\epsilon$, pois as equações diferenciais, que as definem, não envolvem o parâmetro $\epsilon$ (o parâmetro $\epsilon$ aparece em $u_{\epsilon}(t)$ e não em $v(t))$. Logo, a matriz $\Phi_{1}(T)$ terá a seguinte forma:

$$
\Phi_{1}(T)=\left[\begin{array}{cccc}
a_{11}^{1} & 0 & b_{11}^{1} & 0 \\
0 & 0 & 0 & 0 \\
c_{11}^{1} & 0 & d_{11}^{1} & 0 \\
0 & 0 & 0 & 0
\end{array}\right]
$$

Por fim, indicaremos a matriz $\Phi_{0}(T)$ por:

$$
\Phi_{0}(T)=\left[\begin{array}{cccc}
a_{11}^{0} & 0 & b_{11}^{0} & 0 \\
0 & a_{22}^{0} & 0 & b_{22}^{0} \\
c_{11}^{0} & 0 & d_{11}^{0} & 0 \\
0 & c_{22}^{0} & 0 & d_{22}^{0}
\end{array}\right]
$$


Devemos destacar que os coeficientes $a_{11}^{1}, b_{11}^{1}, c_{11}^{1}$ e $d_{11}^{1}$, que aparecem na matriz $\Phi_{1}(T)$ são caracterizados por:

$$
\begin{cases}a_{11}^{1}=\left.\frac{\partial}{\partial \epsilon}\right|_{\epsilon=0} x_{1}(\bar{T}, \epsilon), & c_{11}^{1}=\left.\frac{\partial}{\partial \epsilon}\right|_{\epsilon=0} x_{1}^{\prime}(\bar{T}, \epsilon) \\ b_{11}^{1}=\left.\frac{\partial}{\partial \epsilon}\right|_{\epsilon=0} x_{2}(\bar{T}, \epsilon), & d_{11}^{1}=\left.\frac{\partial}{\partial \epsilon}\right|_{\epsilon=0} x_{2}^{\prime}(\bar{T}, \epsilon)\end{cases}
$$

Essas representações e simplificações matriciais nos permite uma melhor visualização das contas, para o cálculo de $d \bar{G}_{1}$.

\section{A.3 Voltando a derivada de $\bar{G}_{1}$.}

A expressão de $\bar{G}_{1}=\bar{G}_{1}(x, y)$ é dada por:

$$
\begin{aligned}
& \bar{G}_{1}(x, y)=\Pi \Phi_{1}(T)\left(\bar{z}(x, y)-z_{0}\right)+R_{1}(\bar{z}(x, y)) \Pi C \Phi_{0}(T)\left(\bar{z}(x, y)-z_{0}\right)+ \\
& +R_{0}(\bar{z}(x, y)) \Pi C \Phi_{1}(T)\left(\bar{z}(x, y)-z_{0}\right)
\end{aligned}
$$

Analisaremos, separadamente, cada uma dos termos que compõe a soma dessa expressão. Assim, definimos as funções:

$$
\left\{\begin{array}{l}
\alpha_{1}(x, y)=\Pi \Phi_{1}(\bar{T})\left(\bar{z}(x, y)-z_{0}\right), \\
\alpha_{2}(x, y)=R_{1}(\bar{z}(x, y)) \Pi C \Phi_{0}(\bar{T})\left(\bar{z}(x, y)-z_{0}\right), \\
\alpha_{3}(x, y)=R_{0}(\bar{z}(x, y)) \Pi C \Phi_{1}(\bar{T})\left(\bar{z}(x, y)-z_{0}\right) .
\end{array}\right.
$$

Segue que:

$$
\bar{G}_{1}(x, y)=\alpha_{1}(x, y)+\alpha_{2}(x, y)+\alpha_{3}(x, y) .
$$

Devemos notar que, pela definição da seção transversal $\Sigma_{E}^{+}$, em que estamos trabalhando, que é definida, como uma superfície, via a aplicação $\bar{z}$ (equação (3.4)), teremos:

$$
\frac{\partial \bar{z}}{\partial x}(0,0)=e_{1} \text { e } \frac{\partial \bar{z}}{\partial y}(0,0)=e_{3}
$$

Onde $\left\{e_{1}, e_{2}, e_{3}, e_{4}\right\}$ representam os vetores da base canônica do $\mathbb{R}^{4}$.

Vamos aos cálculos das derivadas:

1. O termo $\alpha_{1}$.

Temos: 


$$
\begin{aligned}
& \frac{\partial \alpha_{1}}{\partial x}(0,0)=\Pi \Phi_{1}(\bar{T})\left(\frac{\partial \bar{z}}{\partial x}(0,0)\right)=\Pi \Phi_{1}(\bar{T})\left(e_{1}\right)=\Pi\left(a_{11}^{1}, 0, c_{11}^{1}, 0\right)= \\
& =\left(a_{11}^{1}, c_{11}^{1}\right) .
\end{aligned}
$$

Da mesma forma em relação a derivada com respeito a variável $y$ :

$$
\frac{\partial \alpha_{1}}{\partial y}(0,0)=\Pi \Phi_{1}(T)\left(e_{3}\right)=\Pi\left(b_{11}^{1}, 0, d_{11}^{1}, 0\right)=\left(b_{11}^{1}, d_{11}^{1}\right) .
$$

2. O termo $\alpha_{2}$.

Temos:

$$
\frac{\partial \alpha_{2}}{\partial x}(0,0)=R_{1}\left(z_{0}\right) \Pi C \Phi_{0}(T)\left(e_{1}\right) \text { e } \frac{\partial \alpha_{2}}{\partial y}(0,0)=R_{1}\left(z_{0}\right) \Pi C \Phi_{0}(T)\left(e_{3}\right) .
$$

Devemos observar que $R_{\epsilon}\left(z_{0}\right)=0$, para todo $\epsilon$. De fato, a função $R_{\epsilon}$ representa a diferença entre o período da órbita periódica e o tempo de retorno da aplicação de Poincaré. Assim, como a órbita periódica é a mesma, $\forall \epsilon$, temos $R_{\epsilon}\left(z_{0}\right)=0, \forall \epsilon$. Logo, $R_{1}\left(z_{0}\right)=0$. Segue que:

$$
\frac{\partial \alpha_{2}}{\partial x}(0,0)=0 \text { e } \frac{\partial \alpha_{2}}{\partial y}(0,0)=0
$$

3. O termo $\alpha_{3}$.

As derivadas de $\alpha_{3}$ são calculadas da mesma forma que as derivadas da função $\alpha_{2}$. Teremos:

$$
\frac{\partial \alpha_{3}}{\partial x}(0,0)=0 \text { e } \frac{\partial \alpha_{3}}{\partial y}(0,0)=0
$$

Concluímos então que:

$$
\frac{\partial \bar{G}_{1}}{\partial x}(0,0)=\frac{\partial \alpha_{1}}{\partial x}(0,0) \text { e } \frac{\partial \bar{G}_{1}}{\partial y}(0,0)=\frac{\partial \alpha_{1}}{\partial y}(0,0) .
$$

Portanto, obtemos a derivada $d \bar{G}_{1}(0,0)$.

Proposição A.3.1 A matriz da derivada $d \bar{G}_{1}(0,0)$ é dada por:

$$
d \bar{G}_{1}(0,0)=\left[\begin{array}{ll}
a_{11}^{1} & b_{11}^{1} \\
c_{11}^{1} & d_{11}^{1}
\end{array}\right]
$$


Onde os coeficientes são dados pela equação (A.10).

Estamos interessados em calcular a derivada da função $f_{1}$ em $(0,0)$. Esta, por sua vez, está relacionada a derivada $d \bar{G}_{1}(0,0)$. Diante da proposição anterior, fica a questão de avaliar melhor os coeficientes da equação (A.10). Faremos isso a seguir.

\section{A.4 Análise dos Coeficientes da Equação A.10.}

Faremos a análise dos coeficientes da equação A.10 por partes.

1. Coeficientes $a_{11}^{1}$ e $c_{11}^{1}$.

Temos:

$$
a_{11}^{1}=\left.\frac{\partial}{\partial \epsilon}\right|_{\epsilon=0} x_{1}(T, \epsilon) \text { e } c_{11}^{1}=\left.\frac{\partial}{\partial \epsilon}\right|_{\epsilon=0} x_{1}^{\prime}(T, \epsilon) .
$$

Onde a curva $x_{1}=x_{1}(t, \epsilon)$ é solução da equação (A.6). Transformando esta equação, que é de ordem 2 , numa equação linear de primeira ordem por meio de $x_{1}=x$ e $x_{1}^{\prime}=y$, obtemos:

$$
\left\{\begin{array}{l}
x^{\prime}=y, x(0)=1 \\
y^{\prime}=u_{\epsilon}(t), y(0)=0
\end{array} .\right.
$$

Podemos escrever esta equação de forma matricial por:

$$
\left\{\begin{array}{l}
z^{\prime}=A_{\epsilon}(t) z \\
z(0)=e_{1}
\end{array}\right.
$$

Onde: $A_{\epsilon}(t)=\left[\begin{array}{cc}0 & 1 \\ u_{\epsilon}(t) & 0\end{array}\right]$.

Segue, então, que:

$$
\left(a_{11}^{1}, c_{11}^{1}\right)=\left.\frac{\partial}{\partial \epsilon}\right|_{\epsilon=0} z(T, \epsilon)
$$

Portanto, para determinarmos o vetor $\left(a_{11}^{1}, c_{11}^{1}\right)$, primeira coluna da matriz $d \bar{G}_{1}(0,0)$, precisaremos avaliar a derivada em relação ao parâmetro $\epsilon$ da solução da equação (A.11), calculada no instante $t=T$. Sendo assim, definimos: 


$$
c_{1}(t)=\left.\frac{\partial}{\partial \epsilon}\right|_{\epsilon=0} z(t, \epsilon) .
$$

Lembremos que a curva $t \mapsto c_{1}(t)$ satisfaz:

$$
\begin{aligned}
& c_{1}^{\prime}(t)=\left.\frac{\partial}{\partial t} \frac{\partial}{\partial \epsilon}\right|_{\epsilon=0} z(t, \epsilon)=\left.\frac{\partial}{\partial \epsilon}\right|_{\epsilon=0} \frac{\partial}{\partial t} z(t, \epsilon)=\left.\frac{\partial}{\partial \epsilon}\right|_{\epsilon=0} A_{\epsilon}(t) z(t, \epsilon) \\
& =A_{1}(t) z(t, 0)+\left.A_{0}(t) \frac{\partial}{\partial \epsilon}\right|_{\epsilon=0} z(t, \epsilon)= \\
& =A_{0}(t) c_{1}(t)+A_{1}(t) z(t, 0) .
\end{aligned}
$$

Observação A.4.1 Devemos fazer algumas observações:

- Observemos que $u_{\epsilon}(t)=-\alpha^{2}-2 \epsilon x_{2}^{2}(t)$. Logo, fazendo $\epsilon=0$, obtemos:

$$
A_{0}(t)=\left[\begin{array}{cc}
0 & 1 \\
-\alpha^{2} & 0
\end{array}\right]=A .
$$

Ou seja, a matriz $A_{0}(t)$ é uma matriz constante que indicaremos por $A$.

- Observemos também que:

$$
A_{1}(t)=\left[\begin{array}{cc}
0 & 0 \\
-2 x_{2}^{2}(t) & 0
\end{array}\right] .
$$

Assim, a expressão para a matriz $A_{1}(t)$ depende basicamente da órbita periódica.

- A curva $t \mapsto z(t, 0)$ é solução de:

$$
\left\{\begin{array}{l}
z^{\prime}=A z, \\
z(0)=e_{1}
\end{array} .\right.
$$

Logo, podemos escrever $z(t, 0)=e^{t A} e_{1}$ (na forma exponencial de matriz).

Tendo em vista essas observações e definindo $b_{1}(t)=A_{1}(t) e^{t A} e_{1}$, segue que a $t \mapsto c_{1}(t)$ satisfaz:

$$
\left\{\begin{array}{l}
c_{1}^{\prime}=A c_{1}+b_{1}(t) \\
c_{1}(0)=(0,0)
\end{array}\right.
$$

Pela Fórmula de Variação dos Parâmetros: 


$$
c_{1}(t)=e^{t A}\left(\int_{0}^{t} e^{-s A} b_{1}(s) d s\right)
$$

Segue então:

$$
\left(a_{11}^{1}, c_{11}^{1}\right)=e^{T A}\left(\int_{0}^{T} e^{-s A} b_{1}(s) d s\right)
$$

\section{Coeficientes $b_{11}^{1}$ e $d_{11}^{1}$}

Temos:

$$
b_{11}^{1}=\left.\frac{\partial}{\partial \epsilon}\right|_{\epsilon=0} x_{2}(T, \epsilon) \text { e } d_{11}^{1}=\left.\frac{\partial}{\partial \epsilon}\right|_{\epsilon=0} x_{2}^{\prime}(T, \epsilon)
$$

Onde a curva $x_{2}=x_{2}(t, \epsilon)$ é solução da equação (A.8). Transformando esta equação, que é de ordem 2 , numa equação linear de primeira ordem por meio de $x_{2}=x$ e $x_{2}^{\prime}=y$, obtemos:

$$
\left\{\begin{array}{l}
z^{\prime}=A_{\epsilon}(t) z \\
z(0)=e_{2}
\end{array}\right.
$$

Teremos:

$$
\left(b_{11}^{1}, d_{11}^{1}\right)=\left.\frac{\partial}{\partial \epsilon}\right|_{\epsilon=0} z(T, \epsilon)
$$

Onde $z=z(t, \epsilon)$ é solução de (A.13).

Procedendo de maneira análoga a que foi feita no cálculo dos coeficientes $a_{11}^{1}$ e $c_{11}^{1}$, definindo:

$$
c_{2}(t)=\left.\frac{\partial}{\partial \epsilon}\right|_{\epsilon=0} z(t, \epsilon)
$$

Seguirá que $t \mapsto c_{2}(t)$ é solução de:

$$
\left\{\begin{array}{l}
c_{2}^{\prime}=A c_{2}+b_{2}(t), \\
c_{2}(0)=(0,0)
\end{array}\right.
$$

Onde:

$$
b_{2}(t)=A_{1}(t) e^{t A} e_{2}
$$

Segue da Fórmula de Variação dos Parâmetros: 


$$
c_{2}(t)=e^{t A}\left(\int_{0}^{t} e^{-s A} b_{2}(s) d s\right)
$$

Portanto:

$$
\left(b_{11}^{1}, d_{11}^{1}\right)=e^{T A}\left(\int_{0}^{T} e^{-s A} b_{2}(s) d s\right)
$$

Analisaremos esses coeficientes a partir das igualdades obtidas em (A.12) e (A.14).

Vamos a seguir calcular os elementos envolvidos nas igualdades (A.12) e (A.14). Temos:

\section{A exponencial da matriz $A$}

Sendo $A=A_{0}(t)$, teremos:

$$
A=\left[\begin{array}{cc}
0 & 1 \\
-\alpha^{2} & 0
\end{array}\right]
$$

Com isso, teremos o polinômio característico da matriz $A$, dado por $p_{A}(x)=x^{2}+\alpha^{2}$. Assim, os autovalores de $A$ são $\{i \alpha,-i \alpha\}$. Segue, do processo de obtenção da forma de Jordan da matriz $A$, que vale $A=Q J Q^{-1}$, onde:

$$
Q=\left[\begin{array}{ll}
1 & 0 \\
0 & \alpha
\end{array}\right] \text { e } J=\left[\begin{array}{cc}
0 & \alpha \\
-\alpha & 0
\end{array}\right]
$$

Segue das propriedades da exponencial de matrizes que vale:

$$
e^{t A}=\left[\begin{array}{ll}
1 & 0 \\
0 & \alpha
\end{array}\right]\left[\begin{array}{cc}
\cos t \alpha & \sin t \alpha \\
-\sin t \alpha & \cos t \alpha
\end{array}\right]\left[\begin{array}{ll}
1 & \alpha \\
0 & \frac{1}{\alpha}
\end{array}\right]=\left[\begin{array}{cc}
\cos t \alpha & \frac{1}{\alpha} \sin t \alpha \\
-\alpha \sin t \alpha & \cos t \alpha
\end{array}\right]
$$

Temos então a expressão para as exponenciais que aparecem nas equações (A.12) e (A.14). Por fim, notemos que a inversa é dada por:

$$
e^{-t A}=\left[\begin{array}{cc}
\cos t \alpha & \frac{-1}{\alpha} \sin t \alpha \\
\alpha \sin t \alpha & \cos t \alpha
\end{array}\right]
$$

Os vetores $b_{1}=b_{1}(t)$ e $b_{2}=b_{2}(t)$

$\mathrm{O}$ vetor $b_{1}$ foi definido como $b_{1}(t)=A_{1}(t) e^{t A} e_{1}$. Mas, pelo cálculo da exponencial da matriz $A, e^{t A} e_{1}=(\cos t \alpha,-\alpha \sin t \alpha)$. Logo:

$$
b_{1}(t)=\left[\begin{array}{cc}
0 & 0 \\
u_{1}(t) & 0
\end{array}\right]\left[\begin{array}{c}
\cos t \alpha \\
-\alpha \sin t \alpha
\end{array}\right]=\left[\begin{array}{c}
0 \\
u_{1}(t) \cos t \alpha
\end{array}\right]
$$


Obtemos, assim, o vetor $b_{1}$. Para o cálculo do vetor $b_{2}$, podemos fazer de maneira análoga, com as adaptações necessárias. Assim, $b_{2}(t)=A_{1}(t) e^{t A} e_{2}$. Mas $e^{t A} e_{2}=\left(\frac{1}{\alpha} \sin t \alpha, \cos t \alpha\right)$. Portanto:

$$
b_{2}(t)=\left[\begin{array}{cc}
0 & 0 \\
u_{1}(t) & 0
\end{array}\right]\left[\begin{array}{c}
\frac{1}{\alpha} \sin t \alpha \\
\cos t \alpha
\end{array}\right]=\left[\begin{array}{c}
0 \\
\frac{u_{1}(t)}{\alpha} \sin t \alpha
\end{array}\right] .
$$

Com isso, conseguimos as seguintes expressões:

$$
b_{1}(t)=\left(0, u_{1}(t) \cos t \alpha\right) \text { e } b_{2}(t)=\left(0, \frac{u_{1}(t)}{\alpha} \sin t \alpha\right) .
$$

Tendo em vista que agora temos a expressão para as exponenciais de matrizes e os vetores $b_{1}$ e $b_{2}$ que aparecem nas equações (A.12) e (A.14), podemos tentar melhorar as integrais.

$\mathrm{O}$ vetor $c_{1}=c_{1}(t)$

Na expressão que define $c_{1}=c_{1}(t)$, temos:

$e^{-s A} b_{1}(s)=\left[\begin{array}{cc}\cos s \alpha & \frac{-1}{\alpha} \sin s \alpha \\ \alpha \sin s \alpha & \cos s \alpha\end{array}\right]\left[\begin{array}{c}0 \\ u_{1}(s) \cos s \alpha\end{array}\right]=\left(\frac{-1}{2 \alpha} u_{1}(s) \sin 2 s \alpha, u_{1}(s) \cos ^{2} s \alpha\right)$

Segue então que:

$c_{1}(t)=e^{t A}\left(\frac{-1}{2 \alpha} \int_{0}^{t} u_{1}(s) \sin 2 s \alpha d s, \int_{0}^{t} u_{1}(s) \cos ^{2} s \alpha d s\right)$

$\mathrm{O}$ vetor $c_{2}=c_{2}(t)$

Da mesma forma, temos:

$e^{-s A} b_{2}(s)=\left[\begin{array}{cc}\cos s \alpha & \frac{-1}{\alpha} \sin s \alpha \\ \alpha \sin s \alpha & \cos s \alpha\end{array}\right]\left[\begin{array}{c}0 \\ \frac{u_{1}(s)}{\alpha} \sin s \alpha\end{array}\right]=\left[\begin{array}{c}\frac{-u_{1}(s)}{\alpha^{2}} \sin ^{2} s \alpha \\ \frac{u_{1}(s)}{2 \alpha} \sin 2 s \alpha\end{array}\right]$

Com isso, podemos escrever:

$c_{2}(t)=e^{t A}\left(\frac{-1}{\alpha^{2}} \int_{0}^{t} u_{1}(s) \sin ^{2} s \alpha d s, \frac{1}{2 \alpha} \int_{0}^{t} u_{1}(s) \sin 2 s \alpha d s\right)$

Podemos agora calcular $c_{1}(T)$ e $c_{2}(T)$.

Cálculo de $c_{1}(T)$ e $c_{2}(T)$

Definimos: 


$$
I_{11}=\frac{-1}{2 \alpha} \int_{0}^{T} u_{1}(s) \sin 2 s \alpha d s \text { e } I_{21}=\int_{0}^{T} u_{1}(s) \cos ^{2} s \alpha d s
$$

Segue então que:

$$
c_{1}(T)=e^{T A}\left(I_{11}, I_{21}\right)=\left(a_{11}^{1}, c_{11}^{1}\right)
$$

Da maneira análoga para o cálculo de $c_{2}(T)$, definimos:

$$
I_{12}=\frac{-1}{\alpha^{2}} \int_{0}^{T} u_{1}(s) \sin ^{2} s \alpha d s \text { e } I_{22}=\frac{1}{2 \alpha} \int_{0}^{T} u_{1}(s) \sin 2 s \alpha d s
$$

Portanto:

$$
c_{2}(T)=e^{T A}\left(I_{12}, I_{22}\right)
$$

Essa análise pode ser resumida na próxima proposição.

Proposição A.4.1 A derivada $d \bar{G}_{1}(0,0)$ é dada por:

$$
d \bar{G}_{1}(0,0)=e^{T A} I
$$

Onde:

$$
I=\left[\begin{array}{ll}
I_{11} & I_{12} \\
I_{21} & I_{22}
\end{array}\right] \text { e } A=\left[\begin{array}{cc}
0 & 1 \\
-\alpha^{2} & 0
\end{array}\right]
$$

Com $T$ sendo o período da órbita periódica $\Gamma_{E}$.

Devemos ressaltar que gostaríamos de calcular as derivadas da função $f_{1}$, pois elas estão relacionadas a função $A_{1}$, que deveremos avaliar a soma nas órbitas da aplicação $\psi_{0}$, sobre a curva invariante $\Gamma$. A partir da proposição anterior, temos a derivada de $f_{1}$ em $(0,0) \in \Omega$.

Corolário A.4.1 Vale a igualdade:

$$
d f_{1}(0,0)=e^{T A} I e^{-T A} .
$$

Onde I é a matriz que aparece na proposição anterior.

Demonstração: Segue da proposição anterior e da igualdade (A.1). 


\section{A.5 Cálculo das derivadas de $A_{1}$}

O objetivo, agora, será o de avaliar as derivadas parciais da função $A_{1}$, descritas na proposição 7.5.2. Em virtude do último corolário, temos condições de fazer essa avaliação. Ao longo dessa subseção, definimos a matriz $W$ :

$$
W=\left[\begin{array}{ll}
W_{11} & W_{12} \\
W_{21} & W_{22}
\end{array}\right] \doteq e^{T A} I e^{-T A} .
$$

Observemos que:

$$
\begin{aligned}
& I e^{-T A}=\left[\begin{array}{ll}
I_{11} & I_{12} \\
I_{21} & I_{22}
\end{array}\right]\left[\begin{array}{cc}
\cos T \alpha & \frac{-1}{\alpha} \sin T \alpha \\
\alpha \sin T \alpha & \cos T \alpha
\end{array}\right]= \\
& =\left[\begin{array}{cc}
I_{11} \cos T \alpha+I_{12} \alpha \sin T \alpha & \frac{-I_{11}}{\alpha} \sin T \alpha+I_{12} \cos T \alpha \\
I_{21} \cos T \alpha+I_{22} \alpha \sin T \alpha & \frac{-I_{21}}{\alpha} \sin T \alpha+I_{22} \cos T \alpha
\end{array}\right]
\end{aligned}
$$

Obtemos, então, a seguinte igualdade para a matriz $W$ :

$$
W=\left[\begin{array}{cc}
\cos T \alpha & \frac{1}{\alpha} \sin T \alpha \\
-\alpha \sin T \alpha & \cos T \alpha
\end{array}\right]\left[\begin{array}{cc}
I_{11} \cos T \alpha+I_{12} \alpha \sin T \alpha & \frac{-I_{11}}{\alpha} \sin T \alpha+I_{12} \cos T \alpha \\
I_{21} \cos T \alpha+I_{22} \alpha \sin T \alpha & \frac{-I_{21}}{\alpha} \sin T \alpha+I_{22} \cos T \alpha
\end{array}\right] .
$$

A partir desta igualdade, podemos calcular as entradas $W_{i j}$ da matriz $W$. Seguiremos por partes.

- Cálculo de $W_{11}$.

Observemos que:

$$
W_{11}=I_{11} \cos ^{2} T \alpha+\frac{\alpha I_{12}}{2} \sin 2 T \alpha+\frac{I_{21}}{2 \alpha} \sin 2 T \alpha+I_{22} \sin ^{2} T \alpha .
$$

Assim, podemos escrever:

$$
W_{11}=\left(I_{11} \cos ^{2} T \alpha+I_{22} \sin ^{2} T \alpha\right)+\frac{\left(\alpha^{2} I_{12}+I_{21}\right)}{2 \alpha} \sin 2 T \alpha .
$$

- Cálculo do coeficiente $W_{12}$.

Observemos que:

$$
W_{12}=\frac{-I_{11}}{2 \alpha} \sin 2 T \alpha+I_{12} \cos ^{2} T \alpha-\frac{I_{21}}{\alpha^{2}} \sin ^{2} T \alpha+\frac{I_{22}}{2 \alpha} \sin 2 T \alpha .
$$


Assim, podemos escrever:

$$
W_{12}=\frac{1}{2 \alpha}\left(-I_{11}+I_{22}\right) \sin 2 T \alpha+\frac{1}{\alpha^{2}}\left(\alpha^{2} I_{12} \cos ^{2} T \alpha-I_{21} \sin ^{2} T \alpha\right) .
$$

- Cálculo do coeficiente $W_{21}$.

Procedendo de maneira análoga a que foi feita nos coeficientes $W_{11}$ e $W_{12}$, temos:

$$
W_{21}=\frac{-\alpha I_{11}}{2} \sin 2 T \alpha-\alpha^{2} I_{12} \sin ^{2} T \alpha+I_{21} \cos ^{2} T \alpha+\frac{\alpha I_{22}}{2} \sin 2 T \alpha .
$$

Logo:

$$
W_{21}=\frac{\alpha}{2}\left(-I_{11}+I_{22}\right) \sin 2 T \alpha+I_{21} \cos ^{2} T \alpha-\alpha^{2} I_{12} \sin ^{2} T \alpha .
$$

- Cálculo de $W_{22}$.

Temos:

$$
W_{22}=I_{11} \sin ^{2} T \alpha-\frac{\alpha I_{12}}{2} \sin 2 T \alpha-\frac{I_{21}}{2 \alpha} \sin 2 T \alpha+I_{22} \cos ^{2} T \alpha .
$$

Logo:

$$
W_{22}=I_{11} \sin ^{2} T \alpha+I_{22} \cos ^{2} T \alpha-\frac{\left(\alpha^{2} I_{12}+I_{21}\right)}{2 \alpha} \sin 2 T \alpha .
$$

Observemos que pela definição da matriz $W$, temos:

$$
W_{11}=\frac{\partial X_{1}}{\partial x}(0,0), W_{12}=\frac{\partial X_{1}}{\partial y}(0,0), W_{21}=\frac{\partial Y_{1}}{\partial x}(0,0) \text { e } W_{22}=\frac{\partial Y_{1}}{\partial y}(0,0) .
$$

Diante dessas fórmulas para os coeficientes $W_{i j}$ e as derivadas parciais da função $A_{1}$, que foram calculadas na proposição 7.5.2, teremos:

- Cálculo de $\frac{\partial^{2} A_{1}}{\partial x^{2}}(0,0)$.

Temos:

$$
\frac{\partial^{2} A_{1}}{\partial x^{2}}(0,0)=-2 \alpha^{2} \frac{\partial X_{1}}{\partial x}(0,0)=-2 \alpha^{2} W_{11} .
$$

Simplificando a expressão de $W_{11}$ :

$$
W_{11}=I_{11} \cos 2 T \alpha+\frac{\left(\alpha^{2} I_{12}+I_{21}\right)}{2 \alpha} \sin 2 T \alpha .
$$


Mas:

$$
\frac{\left(\alpha^{2} I_{12}+I_{21}\right)}{2 \alpha}=\frac{-1}{2 \alpha} \int_{0}^{T} u_{1}(s) \sin ^{2} \alpha s d s+\frac{1}{2 \alpha} \int_{0}^{T} u_{1}(s) \cos ^{2} \alpha s d s .
$$

Logo:

$$
\frac{\left(\alpha^{2} I_{12}+I_{21}\right)}{2 \alpha}=\frac{1}{2 \alpha} \int_{0}^{T} u_{1}(s) \cos 2 \alpha s d s .
$$

Segue então que, substituindo esta expressão em $W_{11}$ e utilizando a definição do coeficiente $I_{11}$, conseguimos:

$W_{11}=-\cos 2 T \alpha \frac{1}{2 \alpha} \int_{0}^{T} u_{1}(s) \sin 2 \alpha s d s+\sin 2 T \alpha \frac{1}{2 \alpha} \int_{0}^{T} u_{1} \cos 2 \alpha s d s$

$=\frac{1}{2 \alpha} \int_{0}^{T} u_{1}(s)(\sin 2 \alpha T \cos 2 \alpha s-\cos 2 \alpha T \sin 2 \alpha s) d s=$

$=\frac{1}{2 \alpha} \int_{0}^{T} u_{1}(s) \sin \{2 \alpha(T-s)\} d s$.

Portanto:

$$
\frac{\partial X_{1}}{\partial x}(0,0)=\frac{1}{2 \alpha} \int_{0}^{T} u_{1}(s) \sin \{2 \alpha(T-s)\} d s .
$$

Logo:

$$
\frac{\partial^{2} A_{1}}{\partial x^{2}}(0,0)=-\alpha \int_{0}^{T} u_{1}(s) \sin \{2 \alpha(T-s)\}
$$

- Cálculo de $\frac{\partial^{2} A_{1}}{\partial y^{2}}(0,0)$.

Notemos que $\frac{\partial^{2} A_{1}}{\partial y^{2}}(0,0)=-2 \frac{\partial Y_{1}}{\partial y}(0,0)$. Mas:

$$
\frac{\partial Y_{1}}{\partial y}(0,0)=-\frac{\partial X_{1}}{\partial x}(0,0)=-W_{11} .
$$

Obtemos então:

$$
\frac{\partial^{2} A_{1}}{\partial y^{2}}(0,0)=\frac{1}{\alpha} \int_{0}^{T} u_{1}(s) \sin \{2 \alpha(T-s)\} d s
$$


- Cálculo de $\frac{\partial^{2} A_{1}}{\partial x \partial y}(0,0)$. Observemos que:

$$
\frac{\partial^{2} A_{1}}{\partial x \partial y}(0,0)=-\alpha^{2} \frac{\partial X_{1}}{\partial y}(0,0)-\frac{\partial Y_{1}}{\partial x}(0,0)=-\alpha^{2} W_{12}-W_{21} .
$$

Mas devemos observar que:

$$
-\alpha^{2} W_{12}=\frac{-\alpha}{2}\left(-I_{11}+I_{22}\right) \sin 2 T \alpha+I_{21} \sin ^{2} T \alpha-\alpha^{2} I_{12} \cos ^{2} T \alpha .
$$

Utilizando que $I_{22}=-I_{11}$, conseguimos:

$$
-\alpha^{2} W_{12}=I_{11} \alpha \sin 2 T \alpha+I_{21} \sin ^{2} T \alpha-\alpha^{2} I_{12} \cos ^{2} T \alpha .
$$

Da mesma forma, temos:

$$
-W_{21}=I_{11} \alpha \sin 2 T \alpha+\alpha^{2} I_{12} \sin ^{2} T \alpha-I_{21} \cos ^{2} T \alpha .
$$

Segue então que:

$$
\begin{aligned}
\frac{\partial^{2} A_{1}}{\partial x \partial y}(0,0) & =2 \alpha I_{11} \sin 2 T \alpha+\left(\alpha^{2} I_{12}+I_{21}\right)\left(\sin ^{2} T \alpha-\cos ^{2} T \alpha\right) . \\
& =2 \alpha I_{11} \sin 2 T \alpha-\left(\alpha^{2} I_{12}+I_{21}\right) \cos 2 T \alpha .
\end{aligned}
$$

Utilizando a expressão de $\left(\alpha^{2} I_{12}+I_{21}\right)$ e utilizando a definição do coeficiente $I_{11}$, podemos escrever:

$$
\begin{aligned}
& \frac{\partial^{2} A_{1}}{\partial x \partial y}(0,0)=-\sin 2 T \alpha \int_{0}^{T} u_{1}(s) \sin 2 s \alpha d s-\cos 2 T \alpha \int_{0}^{T} u_{1}(s) \cos 2 s \alpha \\
& d s= \\
& =(-1) \int_{0}^{T} u_{1}(s)(\cos 2 T \alpha \cos 2 s \alpha+\sin 2 T \alpha \sin 2 s \alpha) d s= \\
& =(-1) \int_{0}^{T} u_{1}(s) \cos \{2 \alpha T-2 \alpha s\} d s= \\
& =(-1) \int_{0}^{T} u_{1}(s) \cos \{2 \alpha(T-s)\} d s .
\end{aligned}
$$

Concluímos então:

$$
\frac{\partial^{2} A_{1}}{\partial x \partial y}(0,0)=(-1) \int_{0}^{T} u_{1}(s) \cos \{2 \alpha(T-s)\} d s .
$$

Tendo em vista que $u_{\epsilon}(t)=-\alpha^{2}-2 \epsilon x_{2}^{2}(t)$,onde $t \mapsto\left(0, x_{2}(t), 0, y_{2}(t)\right)$ é a órbita $\Gamma_{E}$, podemos representar as expressões obtidas por meio de integrais sobre $\Gamma_{E}$. Isso conclui a demonstração da proposição 7.6.1. 


\section{Apêndice B}

\section{Análise da subvariedade $M_{2}$}

O objetivo aqui é analisar a subvariedade invariante $M_{2}$, construída no Capítulo 3, visando dar uma melhor descrição da dinâmica do sistema hamiltoniano estudado no texto.

Considerando a Hamiltoniana $H_{\epsilon}: \mathbb{R}^{4} \rightarrow \mathbb{R}$, dada por:

$$
H_{\epsilon}=\frac{y_{1}^{2}+y_{2}^{2}}{2}+\frac{\alpha^{2} x_{1}^{2}}{2}+\frac{b_{1} x_{1}^{4}}{4}-\frac{\nu^{2} x_{2}^{2}}{2}+\frac{b_{2} x_{2}^{4}}{4}-R_{\epsilon}\left(x_{1}, x_{2}\right),
$$

onde $R_{\epsilon}$ é analítica real e tem a seguinte forma:

$$
R_{\epsilon}\left(x_{1}, x_{2}\right)=\epsilon\left(x_{1}^{2} x_{2}^{2}+R_{1}\left(x_{1}, x_{2}\right)\right), \text { com } R_{1}=x_{1}^{3} R_{2}\left(x_{1}, x_{2}\right)
$$

Segue que o campo hamiltoniano é dado por:

$$
X_{H_{\epsilon}}=\left(y_{1}, y_{2},-\alpha^{2} x_{1}-b_{1} x_{1}^{3}+\partial_{x_{1}} R_{\epsilon}, \nu x_{2}-b_{2} x_{2}^{3}+\partial_{x_{2}} R_{\epsilon}\right)
$$

Para uma análise da existência de um horseshoe ${ }^{1}$ para o fluxo do campo Hamiltoniano $X_{H_{\epsilon}}$, procederemos como no exemplo 2.1.4; ou seja, construiremos uma família de campos de vetores $\left(F_{\delta}\right)_{\delta \geq 0}$, tal que os fluxos dos campos $X_{H_{\epsilon}}$ e $F_{\delta}$ são conjugados, para todo $\delta>0$. Para $\delta=0$, a subvariedade $\Sigma^{0}$ é normalmente hiperbólica e suas variedades estável e instável coincidem. Aplicando o método de Melnikov, teremos que para $\delta>0$, as correspondente variedades estável e instável se interceptarão transversalmente, existindo assim um horse-shoe para o fluxo de $F_{\delta}$, para $\delta>0$, pequeno, e assim valendo o mesmo para o campo $X_{H_{\epsilon}}$. O objetivo, neste apêndice, é mostrar que a análise para a Hamiltoniana $H_{\epsilon}$ se reduz a análise feita em [4] e [5], que foi apresentada no exemplo 2.1.4. Procederemos por partes.

\footnotetext{
${ }^{1}$ Neste apêndice, mostraremos que, no caso em que temos o termo geral $R_{\epsilon}$, como apresentado em (1.1), os resultados de [5] e [4] (caso comentado no exemplo 2.1.4) permanecem válidos.
} 
1. Construção do campo $F_{\delta}$.

Para cada $\delta>0$, consideremos a família de aplicações diferenciáveis, $\xi_{\delta}: \mathbb{R}^{4} \rightarrow \mathbb{R}^{4}$, dada por:

$$
\xi_{\delta}\left(x_{1}, x_{2}, y_{1}, y_{2}\right)=\left(\frac{x_{1}}{\sqrt{\delta}}, x_{2}, \frac{y_{1}}{\sqrt{\delta}}, y_{2}\right)
$$

Definimos o campo auxiliar $G_{\delta}(z)=d_{z} \xi_{\delta}\left(X_{H_{\epsilon}}(z)\right)$. Logo:

$$
\begin{gathered}
G_{\delta}\left(x_{1}, x_{2}, y_{1}, y_{2}\right)=\left[\begin{array}{cccc}
\frac{1}{\sqrt{\delta}} & 0 & 0 & 0 \\
0 & 1 & 0 & 0 \\
0 & 0 & \frac{1}{\sqrt{\delta}} & 0 \\
0 & 0 & 0 & 1
\end{array}\right]\left[\begin{array}{c}
y_{1} \\
y_{2} \\
-\alpha^{2} x_{1}-b_{1} x_{1}^{3}+\partial_{x_{1}} R_{\epsilon} \\
\nu^{2} x_{2}-b_{2} x_{2}^{3}+\partial_{x_{2}} R_{\epsilon}
\end{array}\right] \\
=\left(\frac{y_{1}}{\sqrt{\delta}}, y_{2}, \frac{-\alpha^{2} x_{1}-b_{1} x_{1}^{3}+\partial_{x_{1}} R_{\epsilon}}{\sqrt{\delta}}, \nu^{2} x_{2}-b_{2} x_{2}^{3}+\partial_{x_{2}} R_{\epsilon}\right) .
\end{gathered}
$$

Por fim, definimos o campo $F_{\delta}$, para $\delta>0$, por:

$$
F_{\delta}(z)=G_{\delta}\left(\xi_{\delta}^{-1}(z)\right)=d_{\xi_{\delta}^{-1}} \xi_{\delta}\left(X_{H_{\epsilon}}\left(\xi_{\delta}^{-1}(z)\right)\right)
$$

Dessa forma, $\xi_{\delta}$ é uma conjugação entre os fluxos de $X_{H_{\epsilon}}$ e $F_{\delta}$. O campo $F_{\delta}$ é dado por:

$$
F_{\delta}=\left(y_{1}, y_{2},-\alpha^{2} x_{1}-b_{1} \delta x_{1}^{3}+\frac{\partial_{x_{1}} R_{\epsilon}}{\sqrt{\delta}}\left(\sqrt{\delta} x_{1}, x_{2}\right), \nu^{2} x_{2}-b_{2} x_{2}^{3}+\partial_{x_{2}} R_{\epsilon}\left(\sqrt{\delta} x_{1}, x_{2}\right)\right)
$$

Sendo $R_{\epsilon}=\epsilon\left(x_{1}^{2} x_{2}^{2}+x_{1}^{3} R_{2}\left(x_{1}, x_{2}\right)\right)$, teremos:

- $\frac{\partial R_{\epsilon}}{\partial x_{1}}=2 \epsilon x_{1} x_{2}^{2}+3 \epsilon x_{1}^{2} R_{2}+\epsilon x_{1}^{3} \frac{\partial R_{2}}{\partial x_{1}} ;$ Logo:

$$
\frac{\partial_{x_{1}} R_{\epsilon}}{\sqrt{\delta}}\left(\sqrt{\delta} x_{1}, x_{2}\right)=2 \epsilon x_{1} x_{2}^{2}+\epsilon 3 \sqrt{\delta} R_{2}\left(\sqrt{\delta} x_{1}, x_{2}\right)+\epsilon \delta^{\frac{3}{2}} x_{1}^{3} \frac{\partial R_{2}}{\partial x_{1}}\left(\sqrt{\delta} x_{1}, x_{2}\right) .
$$

- Procedendo de maneira análoga a derivada com respeito a $x_{2}$, obtemos:

$$
\partial_{x_{2}} R_{\epsilon}\left(\sqrt{\delta} x_{1}, x_{2}\right)=2 \epsilon \delta x_{2} x_{1}^{2}+\epsilon \delta^{\frac{3}{2}} x_{1}^{3} \frac{\partial R_{2}}{\partial x_{1}}\left(\sqrt{\delta} x_{1}, x_{2}\right)
$$


Observemos que se a função $R_{2}$ tiver a forma $R_{2}\left(x_{1}, x_{2}\right)=x_{1}^{k_{1}} x_{2}^{k_{2}}$, para $k_{1}$ e $k_{2}$ suficientemente grandes, então o campo $F_{\delta}$ depende diferenciavelmente do parâmetro $\delta$ e $F_{\delta}$ está definido para $\delta=0$. Assumiremos que estamos nessa situação. Para $\delta=0$, temos então:

$$
F_{0}\left(x_{1}, x_{2}, y_{1}, y_{2}\right)=\left(y_{1}, y_{2},-\alpha^{2} x_{1}+2 \epsilon x_{1} x_{2}^{2}, a_{2} x_{2}-b_{2} x_{2}^{3}\right)
$$

2. O comportamento do campo $F_{0}$.

Observemos que, em relação ao campo $F_{0}$, o comportamento nas variáveis $\left(x_{2}, y_{2}\right)$ não dependem das variáveis $\left(x_{1}, y_{1}\right)$. Consideremos o conjunto:

$$
M_{2}=\left\{\left(x_{1}, x_{2}, y_{1}, y_{2}\right) \in \mathbb{R}^{4}: x_{2}=y_{2}=0\right\}
$$

Esta é uma subvariedade invariante pelo fluxo do campo $F_{0}$, que é normalmente hiperbólica. Determinemos suas variedades estável e instável, indicadas por $W_{0}^{+}(S)$ e $W_{0}^{-}(S)$, respectivamente. Consideremos a função $h: \mathbb{R}^{4} \rightarrow \mathbb{R}$, definida por:

$$
h\left(x_{1}, x_{2}, y_{1}, y_{2}\right)=\frac{y_{2}^{2}}{2}-\frac{\nu^{2} x_{2}^{2}}{2}+\frac{b_{2} x_{2}^{4}}{4},
$$

essa função $h$ é uma integral primeira do campo $F_{0}$. Além disso, é possível verificar que vale:

$$
W_{0}^{+}(S)=W_{0}^{-}(S)=h^{-1}(0) .
$$

A subvariedade $\Sigma^{0}$ permanece invariante e normalmente hiperbólica para $\delta>0$. Indiquemos as subvariedades instável e estável, associadas, por $W_{\delta}^{-}(S)$ e $W_{\delta}^{+}(S)$, respectivamente. Gostaríamos de avaliar a interseção entre elas, para $\delta>0$ pequeno e não nulo. Podemos proceder como em [5] e [4]. Assim, a análise será feita através da função de Melnikov $m: W_{0}^{ \pm}(S) \rightarrow \mathbb{R}$, dada por:

$$
m(p)=\int_{\mathbb{R}}\langle\nabla h, \bar{Y}\rangle \circ \phi_{t}^{F_{0}}(p) d t .
$$

Onde $\bar{Y}=\left.\frac{\partial}{\partial \delta} \frac{\partial}{\partial t}\right|_{t, \delta=0} \phi_{t}^{F_{0}}$.

3. O fluxo do campo $F_{0}$ sobre $W_{0}^{ \pm}(S)$.

O fluxo de $F_{0}$ sobre $W_{0}^{ \pm}(S)$ pode ser representado por: 


$$
\begin{cases}x_{2}(t)= \pm \sqrt{\frac{2 a_{2}}{b_{2}}} \operatorname{sech}\left(\sqrt{a_{2}}\left(t+t_{0}\right)\right), & y_{2}(t)=\mp a_{2} \sqrt{\frac{2}{b_{2}}} \operatorname{tangh}\left(\sqrt { a _ { 2 } } ( t + t _ { 0 } ) \operatorname { s e c h } \left(\sqrt{a_{2}}(t+\right.\right. \\ x_{1}(t)=c_{0} X_{0}\left(t+t_{0}\right)+c_{1} X_{1}\left(t+t_{0}\right), & y_{1}=\dot{x}_{1}(t)\end{cases}
$$

Onde $\left\{X_{0}, X_{1}\right\}$ é uma base do espaço de soluções da seguinte equação diferencial:

$$
x^{\prime \prime}=-\alpha^{2} x+2 \epsilon x x_{2}^{2}
$$

Fazendo $q(t)=\epsilon x_{2}^{2}(t)=\frac{2 \epsilon \nu^{2}}{b_{2}} \operatorname{sech}^{2}\left(\nu\left(t+t_{0}\right)\right)$, obtemos que $\left\{X_{0}, X_{1}\right\}$ é base do espaço das soluções da seguinte equação:

$$
x^{\prime \prime}+\left\{\alpha^{2}-2 q(t)\right\} x=0
$$

4. A função de Melnikov a partir da expressão do fluxo de $F_{0}$ sobre $W_{0}^{ \pm}(S)$.

Observemos que:

$$
\langle\nabla h, \bar{Y}\rangle=2 \epsilon x_{2} y_{2} x_{1}^{2}
$$

Dessa forma, teremos:

$$
\langle\nabla h, \bar{Y}\rangle \circ \phi_{t}^{F_{0}}=\dot{q}(t)\left(c_{0}^{2} X_{0}^{2}+2 c_{0} c_{1} X_{0} X_{1}+c_{1}^{2} X_{1}^{2}\right)
$$

Portanto, podemos escrever:

$$
m(p)=m_{00} c_{0}^{2}+2 m_{01} c_{0} c_{1}+m_{11} c_{1}^{2}, \operatorname{com} m_{i j}=\int_{\mathbb{R}} \dot{q}(t) X_{i}(t) X_{j}(t) d t
$$

Observando que a função $q=q(t)$, definida neste texto, tem as mesmas propriedades que a função $q$, que é utilizada em [4], a situação se torna análoga a análise feita em [5] e [4]. 


\section{Referências Bibliográficas}

[1] S. Angenent. A remark on the topological entropy and invariant circles of an area preserving twist map. Twist mappings and their applications, edited by R.McGeehee and K.R.Meyer. 1-5, IMA Vol. Math. Appl., 44, Springer, 1992.

[2] M. C. Arnaud. Hyperbolicity for conservative twist maps of the 2dimensional annulus. hal-01174964, 2015.

[3] V. I. Arnold. Mathematical Methods of Classical Mechanics. GTM, Springer, 1978.

[4] L. Butler. Positive-entropy Hamiltonian sustems on Nilmanifolds via scattering. Nonlinearity, 2014.

[5] L. Butler and V. Gelfreich. Positve-entropy geodesic flows on nilmanifolds. Nonlinearity, 2008.

[6] P. Le Calvez. Propriétés dynamiques des régions d'instabilité. Ann. Sci. Éscole Norm. Sup, no. 3, 443-464, 1987.

[7] G. Contreras. Geodesic flows with positive topological entropy, twist maps and hyperbolicity. Annals of Mathematics, 172 (2010), 2010.

[8] G. Contreras and G. Paternain. Genericity of geodesic flows with positive topological entropy on S2. J. Differential Geom. 61 (2002), no. 1, 1-49, 2002.

[9] J. Damasceno, M. Carneiro, and R. Ramirez Ros. The billiard inside an ellipse deformed by the curvature flow. Proc. Amer. Math. Soc. 145 (2017), 705-719, 2017.

[10] S. Pinto de Carvalho and Sylvie Oliffson Kamphorst. The first Birkhoff coefficient and the stability of 2-periodic orbits on billiards. Experimental Mathematics Volume 14 Edição 3 Páginas 299-306, 2005. 
[11] N. de Paulo. Sistemas de seções transversais próximos a níveis críticos de sistemas Hamiltonianos em R4. Tese (Doutorado)-IME-USP, 2014.

[12] N. de Paulo and P. Salomão. Systems of transversal sections near critical energy levels of Hamiltonian systems in R4. Memoirs of AMS, 2018.

[13] N. de Paulo and P. A. Salomão. On the multiplicity of periodic orbits and homoclinic near critical energy levels of Hamiltonian systems in $R 4$. Transactions of AMS, 2018.

[14] Christophe Golé. Symplectic twist maps: global variational techniques. World Scientific, Volume 18, 2001.

[15] U. Hryniewicz and P. Salomão. Uma introdução à geometria de contato e aplicações à dinâmica Hamiltoniana. IMPA, 2009.

[16] A. Katok and B. Hasselblat. Introduction to the modern theory of $d y$ namical system. Cambridge University Press, 1995.

[17] Derek F. Lawden. Elliptic Functions and Applications. Applied Mathematical Sciences, 80, 1989.

[18] L. M. Lerman. Hamiltonian systems with loops of a separatrix of a saddles-center. Sel. Math Sov. 10, 297-306, 1991.

[19] A. O. Lopes. Introdução à Mecânica Clássica. EDUSP, 2006.

[20] J. N. Mather. Variational construction of orbits of twist diffeomorphisms. J. Amer. Math. Soc., 1991.

[21] D. McDuff and D. Salamon. Introduction to symplectic topology. Oxford University Press, 1998.

[22] R. Moeckel. Generic birfurcations of the twist coefficient. Erg. Th. Dyn. Syst. 10, 185-195, 1990.

[23] C. Ragazzo, M. Carneiro, and S. Zanata. Uma Introdução às Aplicações do Tipo Twist. IMPA, 2005.

[24] C. G. Ragazzo. Irregular Dynamics and Homoclinic Orbits to Hamiltonian Saddle Centers. Comm. Pure App. Math. L, 105-147, 1997.

[25] C. G. Ragazzo. On the stability of double homoclinic loops. Comm. Math. Phys. 184, 251-272, 1997. 
[26] C. G. Ragazzo. Stability of homoclinic orbits and diffusion in phase space. Physics Letters., 1997.

[27] C. G. Ragazzo and S. Addas-Zanatta. Critical number in scattering and escaping problems in classical mechanics. Physical Review E 64, 2001.

[28] R. Ramirez-Ros and S. Pinto de Carvalho. Non-persistence of resonant caustics in perturbed elliptic billiards. Journal Ergodic Theory and Dynamical Systems Volume 33 Issue 6 Pages 1876-1890 Publisher Cambridge University Press, 2013.

[29] R. Ramirez-Ros and A. Delshams. Melnikov potential for exact symplectic maps. Communications in mathematical physics, Pages 213-245 Publisher Springer-Verlag, 1997.

[30] P. Salomão. Convex energy levels of Hamiltonian systems. Qualitative Theory of Dynamical Systems, 2004.

[31] A. C. Silva. Lectures on Symplectic Geometry. Lectures Notes in Mathematics, 1764. Springer-Verlag, Berlin., 2001.

[32] M. G. Soares. Cálculo em Uma Variável Complexa. Coleção Matemática Universitária, IMPA, 2009.

[33] J. Sotomayor. Lições de Equações Diferenciais Ordinárias. Coleção projeto Euclides, CNPq, 1979.

[34] E. Zehnder. Homoclinic points near elliptic fixed points. Comm Pure Appl Math., 1973.

[35] E. Zehnder. Lecturs on Dynamical Systems. EMS, 1986. 